\title{
Domain wall dynamics in ferromagnetic nanostructures
}

\section{Maddu Ramu}

School of Physical and Mathematical Sciences

A thesis submitted to the Nanyang Technological University in partial fulfilment of the requirement for the degree of

Doctor of Philosophy 


\section{Acknowledgement}

There are many people who I would like to thank for their support, encouragement during my $\mathrm{PhD}$. Firstly, I would like to express my sincere gratitude to my advisor Prof. Lew Wen Siang for giving me an opportunity to pursue $\mathrm{PhD}$ in his group. I deeply appreciate the amount of time and energy he has spent in helping me hone my research skills, improve my writing and presentation skills. I would also like to extend my gratitude to my co-supervisor Prof. Ivan Shelykh for his continuous support and guidance. I am so grateful for their patience, motivation, and immense knowledge which have made the $\mathrm{PhD}$ journey a truly enriching experience.

I would like to thank Dr. S. Goolaup for his continuous support during the crucial starting and ending days of my $\mathrm{PhD}$. He helped me in developing the skills in nanofabrication and MFM imaging. He also helped in improving my writing skills and also analyzing the results.

I would like to thank Dr. Chandra for all the assistance he has given me during my crucial times of my PhD. I would like to thank Dr. Indra for his help in the starting days of my $\mathrm{PhD}$. He is the one who helped me developing the skills in micromagnetic simulations. I am grateful to my group members Sachin, Pankaj, Gerard, Weiliang, Feilong, Christian for their help in many ways.

I would like to extend my thanks to my parents for their love, support and blessings. Last but not the least, I would like to thank my wife for her immense support during my $\mathrm{PhD}$ studies. 


\section{Table of contents}

Acknowledgements $\quad$ i

Table of contents ii

Summary vii

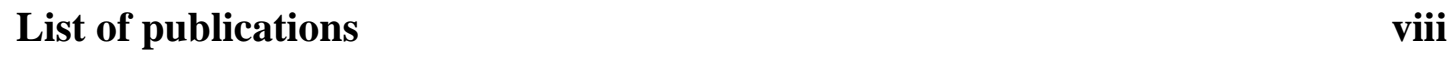

\section{Chapter 1: Introduction}

1.1 Introduction to magnetic storage and logic devices-----------------------------1

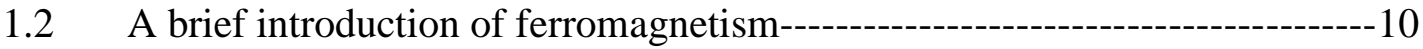

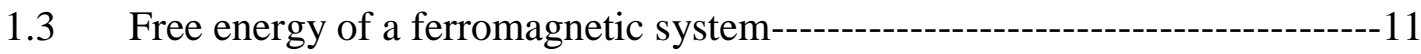

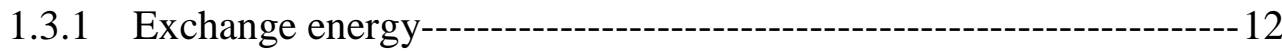

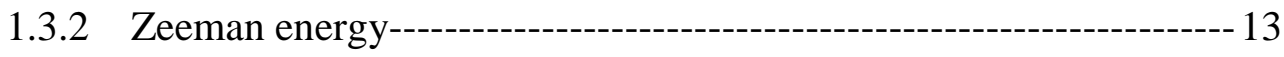

1.3.3 Magneto crystalline anisotropy energy-----------------------------14

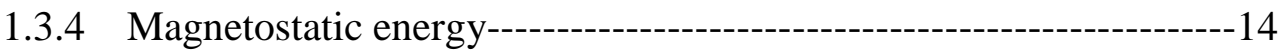

1.3.5 Dzyaloshinskii-Moriya interaction---------------------------------------15

1.4 Magnetic domain and domain walls------------------------------------------------16

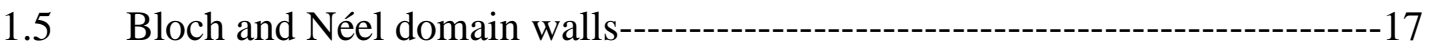

1.6 Transverse and vortex domain walls-----------------------------------------------18

1.7 Magnetic charge possessed by a transverse domain wall------------------------19

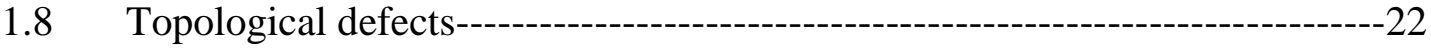

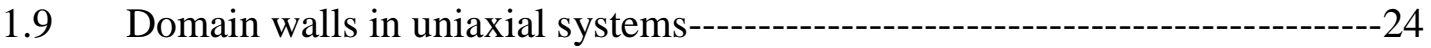

1.10 Magnetization dynamics: Landau-Lifshitz-Gilbert equation--------------------25

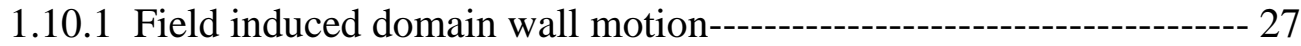




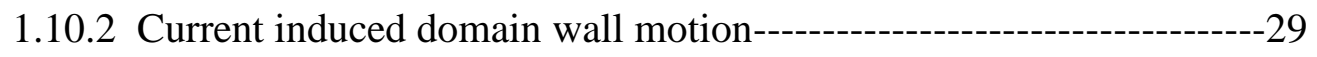

1.10.3 Spin-orbit torque driven domain wall motion--------------------------30

1.10.3.1 Field-like torque---------------------------------------------------31

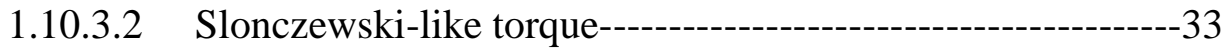

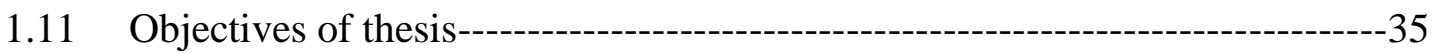

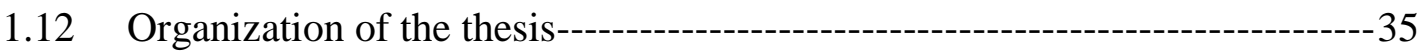

References--------------------------------------------------------------------------------------37

\section{Chapter 2: Experimental techniques}

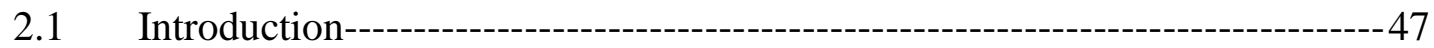

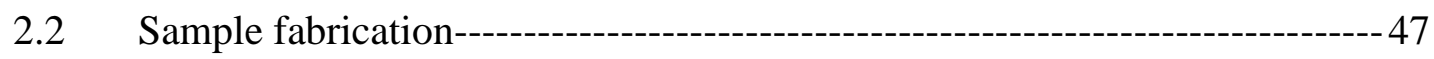

2.3 Thin film deposition by magnetron sputtering-----------------------------------48

2.4 Device fabrication: Electron beam lithography technique-----------------------51

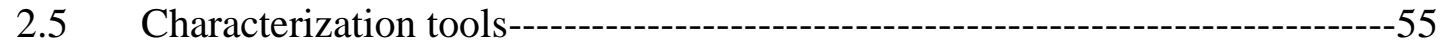

2.5.1 Scanning electron microscopy-------------------------------------------55

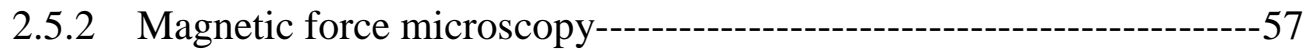

2.5.3 Probe station measurement setup----------------------------------------59

2.5.3.1 Domain wall injection setup---------------------------------------61

2.5.3.2 Domain wall driving setup----------------------------------------62

2.5.4 Method to measure strength and sign of DMI---------------------------63

2.6 Micromagnetic simulations------------------------------------------------------------65

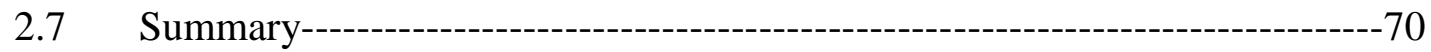

References----------------------------------------------------------------------------------------72

Chapter 3: Transverse domain wall profile for logic applications

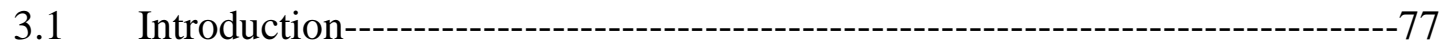




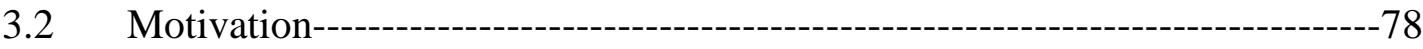

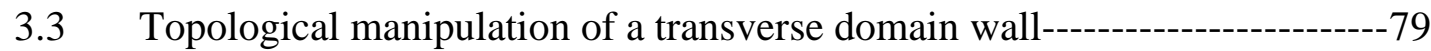

3.3.1 Topological rectifier: Micromagnetic simulations----------------------80

3.3.2 Experimental verification of rectifier----------------------------84

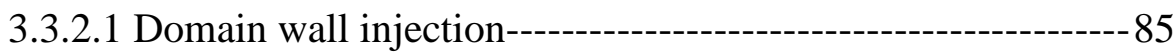

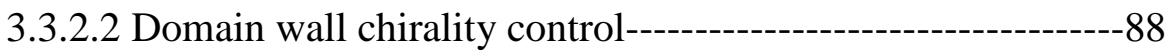

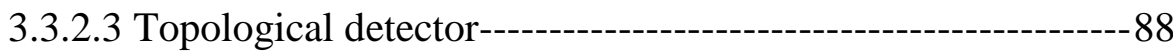

3.3.2.4 Topological rectifier--------------------------------------------92

3.3.3 Topological inverter: Micromagnetic simulations---------------------94

3.3.3.1 Experimental verification of inverter------------------------97

3.3.4 Reliability of the rectification/inversion operation---------------100

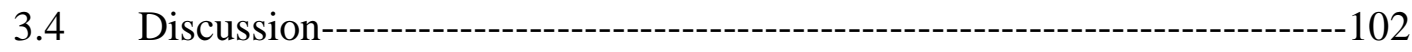

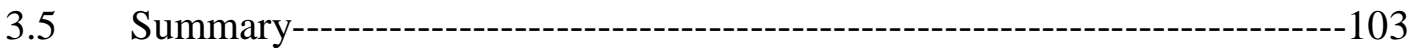

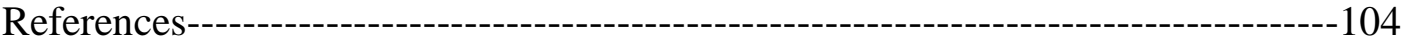

Chapter 4: Edge defect mediated transverse domain wall interactions

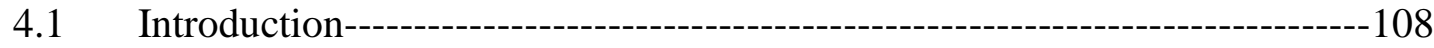

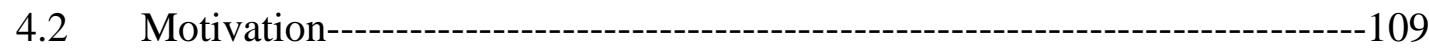

4.3 Deterministic generation of single domain wall

4.3.1 Sample fabrication----

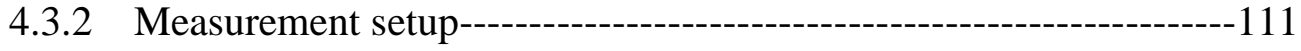

4.3.3 Stochastic domain wall generation-----------------------------------112

4.3.4 Single domain wall generation---------------------------------115

4.3.5 Current induced single domain wall motion-----------------------117

4.3.6 Micromagnetic simulations----------------------------------------------118 
4.4 Spin wave emission via domain wall annihilation-------------------------------121

4.4.1 Effect of nanowire width on spin wave characteristics---------------- 126

4.4.2 Effect of thickness on spin wave characteristics------------------------128

4.4.3 Effect of in-plane field on spin wave characteristics------------------129

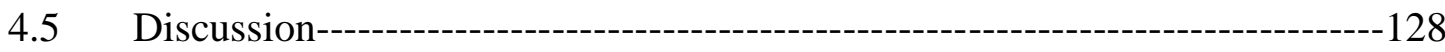

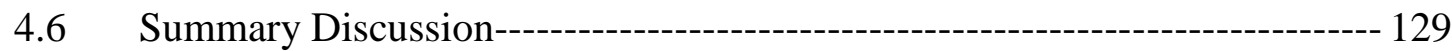

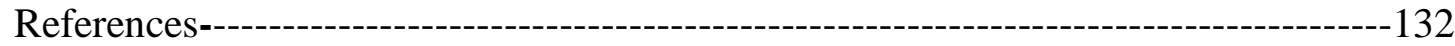

Chapter 5: Spin -orbit torque driven chiral Néel domain wall depinning in $\mathrm{Co} / \mathrm{Ni}$

heterostructures

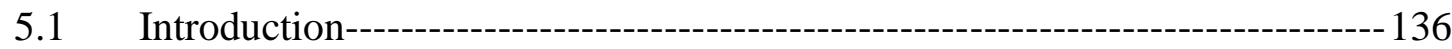

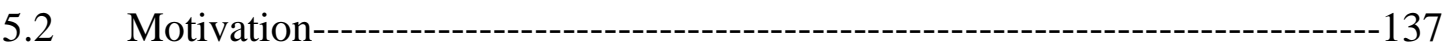

5.3 Current assisted domain wall depinning through Hall cross

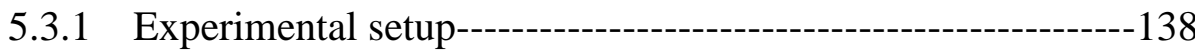

5.3.2 Domain wall injection and detection--------------------------- 141

5.3.3 Current assisted domain wall depinning------------------------ 142

5.3.4 Estimation of the strength of DMI effective field--------------144

5.3.5 Quantification of current induced effective fields-------------- 147

5.3.6 Effect of DMI and SOT on the domain wall depinning-------151

5.3.7 Direct observation of domain wall tilting---------------------154

5.3.8 Effect of Hall cross spacing on domain wall depinning-------156

5.3.9 Dependence of the nanowire width on depinning field------- 157

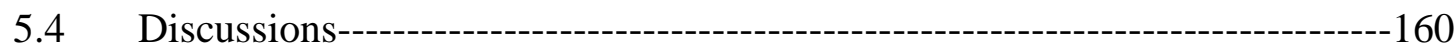

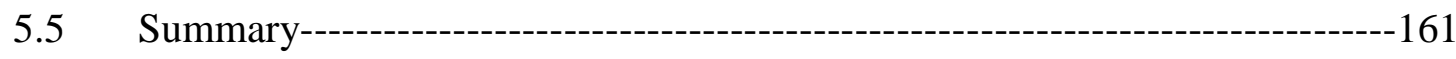

References-------------------------------------------------------------------------------------------162 


\section{Chapter 6: Conclusions}

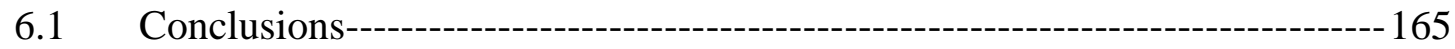

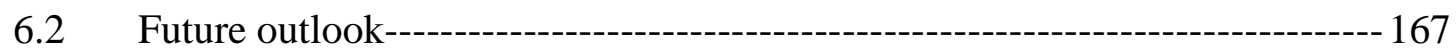

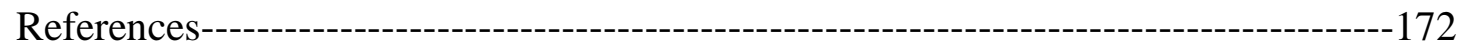




\section{Summary}

Topological defects of domain walls (DWs) in ferromagnetic materials are of paramount importance as regards to both fundamental physics as well as potential applications in non-volatile memory and logic devices. This thesis investigates topological manipulation and dynamics in both in-plane and out-of-plane anisotropy nanostructures. For thin narrow nanowires with in-plane anisotropy, a technique to control, detect and rectify the topological nature of a transverse DW was devised. A rectifier provides a fixed topological output irrespective of the type of input transverse DW while an inverter (Logical NOT) always provides an output which is complimentary to the input. Additionally, topological defects were shown to play a crucial role in DWs interaction, leading to mutual annihilation or formation of bound states. Mutual annihilation further leads to spin wave emission. As such, these effects make the DW generation stochastic. Therefore, a method whereby deterministic generation of single DW in the absence of an external magnetic field and long current pulses was proposed and experimentally verified. The dynamics of chiral Néel DWs driven by spin-orbit torque (SOT) in perpendicularly magnetized $\mathrm{Co} / \mathrm{Ni}$ heterostructures was further investigated. A cross over from the field dominated DW depinning to current dominated domain wall depinning was observed as the magnitude of the current was increased. For field dominated domain wall depinning, both Up-Down and Down-Up DWs exhibit the same depinning efficiencies. However, for current dominated DW depinning, different depinning efficiencies for Up-Down and Down-Up DWs were observed. The difference in depinning fields for Up-Down and Down-Up DWs was found to be increased as the magnitude of the bias current was increased. The interplay between SOT and Dzyaloshinskii-Moriya interaction (DMI) was attributed to this anomaly. 


\section{List of Publications}

\section{International refereed journal}

1. M. Ramu, S. Goolaup, S. Krishnia, G. J. Lim and W. S. Lew, “Spin-orbit torque induced asymmetric depinning of chiral Néel domain wall in Co/Ni heterostructures”, Applied Physics Letters 110, 162402 (2017).

2. M. Ramu, I. Purnama, S. Goolaup, M. C. Sekhar and W. S. Lew, “Investigation of dominant spin wave modes by domain wall collision", Journal of Applied Physics 115, 243908 (2014).

3. S. Goolaup, M. Ramu, M. C. Sekhar and W. S. Lew, "Transverse domain wall profile for logic applications", Scientific Reports 5, 9603 (2015).

4. C. Guite, I. S. Kerk, M. C. Sekhar, M. Ramu, S. Goolaup and W. S. Lew,” Allelectrical deterministic single domain wall generation for on-chip applications", Scientific Reports 4, 7459 (2015).

5. M. Chandra Sekhar, P. Sethi, S. Goolaup, M. Ramu and W. S. Lew, "Direct observation of domain wall evolution at a bifurication in magnetic network structure", Applied Physics Express 7, 113003 (2014).

6. S. Krishnia, P. Sethi, W. L. Gan, F. N. Kholid, M. Ramu, T. S. Herng, J. Ding, I. Purnama and W. S. Lew", Role of RKKY torque on domain wall motion in synthetic antiferromagnetic nanowires with opposite spin Hall angles", (Under review Scientific Reports 2017). 
7. P. Sethi, S. Krishnia, W. L. Gan, F. N. Kholid, F. N. Tan, M. Ramu and W. S. Lew, "Bi-directional high speed domain wall motion in perpendicular magnetic anisotropy Co/Pt double stack nanowires", (Under review Scientific Reports 2017).

\section{PATENTS}

8. "Programmable logic operation via domain wall profile manipulation", S. Goolaup, H. K. Teoh, M. Ramu, M. Chandra Sekhar, I. Purnama, and W. S. Lew, US Provisional Patent 62/101/491 (2015).

\section{CONFERENCE PRESENTATIONS}

1. "Spin wave generation due to domain wall collision" M. Ramu, I. Purnama, M. Chandra Sekhar and W. S. Lew, presented at the ISAMMA Conference, Taiwan (2013).

2. “Transverse domain wall chirality rectifier” M. Ramu, S. Goolaup, M. Chandra Sekhar, P. Sethi, C. K. Guite and W. S. Lew, presented at the IEEE International Magnetics Conference, Germany (2014).

3. "Topological manipulation of magnetic domain wall profile for logic applications" M. Ramu, S. Goolaup, M. Chandra Sekhar, and W.S. Lew, presented at the IEEE International Magnetics Conference, PR China (2015).

4. “Manipulation of transverse domain wall profile for logic Devices" M. Ramu, S. Goolaup, M. Chandra Sekhar, and W.S. Lew, presented at the 20th International Conference on Magnetism, Spain (2015). 
5. "Domain wall annihilation via phase correction in cylindrical nanowires" $\mathbf{M}$. Ramu, S. Goolaup and W. S. Lew, presented at the 20th International Conference on Magnetism, Spain (2015).

6. “A NOT logic operation via domain wall profile manipulation” S. Goolaup, M. C. Shekar, M. Ramu and W. S. Lew, presented at the $4^{\text {th }}$ International Conference on Superconductivity and Magnetism, Turkey (2014).

7. "Fast and deterministic generation of single $D W$ in in-plane ferromagnetic nanowire using local oersted field" C. Guite, K. Inn Seng, M. C. Sekhar, M. Ramu, S. Goolaup and W. S. Lew, presented at the 20th International Conference on Magnetism, Spain (2015).

8. “Transverse domain wall chirality detector” P. Sethi, M. Chandra Sekhar, S. Goolaup, M. Ramu and W. S. Lew, presented at the IEEE International Magnetics Conference, Germany (2014).

9. “Spin-orbit torque induced high speed domain wall motion in Co/Pt dual stack", P. Sethi, S. Krishnia, W. L. Gan, F. N. Kholid, M. Ramu, C. Yunjie, L. S. Huei, W. S. Lew, (MMM 2016 New Orleans, USA).

10. "Effect of the interlayer exchange torque on the domain wall dynamics in perpendicularly magnetized synthetic antiferromagnetic nanowires” S. Krishnia, P. Sethi, W. L. Gan, F. N. Kholid, I. Purnama, M. Ramu, T. S. Herng, J. Ding and W. S. Lew, presented at the $61^{\text {st }}$ Magnetism and Magnetic Materials Conference, USA (2016). 
11. "Skyrmion pinning dynamics in nanostructures for diode and symmetric operations", H. T. Fook, W. L. Gan, I. Purnama, M. Ramu, W. S. Lew, presented at the 20th International Conference on Magnetism, Spain (2015).

12. "Engineering $1 D$ potential barrier for fast guided skyrmion motion”, W. L. Gan, I. Purnama, H. T. Fook, M. Ramu, W. S. Lew, presented at the 20th International Conference on Magnetism, Spain (2015). 


\section{Chapter 1}

\section{Introduction}

\subsection{Introduction to magnetic storage and logic devices}

Semiconductor technology has been growing rapidly since the invention of transistor with advancements in the performance and miniaturizing the size of devices [1-3]. From the earliest punch card which requires an access time of nearly $1 \mathrm{sec}$ for the storage of only $1 \mathrm{kB}$ memory to a modern flash memory that can attain a speed of nearly Gbps, all semiconductor devices work by moving electrical charges [4]. Today's computers often use four kinds of storage; magnetic memories such as hard disk drives (HDDs) and semiconductor memories including dynamic random access memory (DRAM), static random access memory (SRAM), and flash memories at different levels of memory hierarchy [2]. Continued downscaling of these memories is reaching its fundamental limits, where several issues such as high leakage current and heat dissipation start to arise [5]. Nonvolatile HDDs have high storage capacity but rely on moving parts which impose size and speed limitations; hence they remain at the bottom of memory hierarchy [6]. At the top of the memory hierarchy, SRAM has highest speed of about $1 \mathrm{~ns}$; but it lacks in storage capacity, dissipates very high leakage current and volatile in nature. Dynamic random access memory (DRAM) has high storage density but needs to be constantly refreshed and consumes lot of power. Flash memories, unlike SRAM and DRAM, are non-volatile but suffers from excess write power, low write speed, reliability and endurance issues.

In the quest for alternative technologies, spin based devices, known as "spintronic" devices, have emerged as promising candidates [7-9]. Spintronic devices utilize electron's spin degree of freedom as a state variable for computation. Spin can take one of the two 
possible states, "up" and "down", with respect to a magnetic field. These two states can be represented as two binary data bits of digital information: "1" and "0". Spintronic devices can outperform the conventional semiconductor devices due to their unique features such as non-volatility, ultrahigh dense packing and low power. The demonstration of current induced magnetization switching of a nanomagnet has led to several novel spintronic devices, such as MRAM (Magnetic Random Access Memory) and STT-MRAM (Spin Transfer Torque MRAM) [10-13]. MRAM, unlike DRAM which stores the data in electric charge flow, stores the information by magnetic storage elements [14]. MRAM comprises of memory cells which consists of a magnetic tunnel junction (MTJ); a sandwich of thin layers of ferromagnetic materials separated by an ultrathin layer of an insulating material (typically an oxide material). When the magnetizations of two ferromagnetic layers are aligned in the same direction, the electrical resistance across the junction decreases and when the magnetizations are aligned in different directions, the resistance becomes very high. MRAM uses the change in resistance between the two states to encode the information. It is believed that as MRAM improves, it can combine all the advantages of SRAM, DRAM, flash and hard disk drives. It would features high speed, low power, greater scalability and non-volatile. However, current MRAM designs suffers from mainly two issues; cost per bit is very high and writing a bit is performed by applying a magnetic field which imposes limitations on the size [15]. On the other hand, STT-MRAM uses a bidirectional current to switch the magnetization via STT effect [16]. However, STT-MRAM poses serious reliability and endurance problems. This is due to the rapid aging of tunnel barrier induced by high current densities and also erroneous writing by read current [17]. These issues make the realization of STT-MRAM a challenging task, particularly for the 
applications where high speed and scalability are crucial [18]. Currently, a lot of research has been devoted to address these technological issues and to commercialize as a viable memory product. As a subsidiary technology, domain wall (DW) based memory and logic devices have gained lot of research interest. The DW based memory device features ultrahigh storage density, fast data access and non-volatility.

One of the key challenges for the realization of DW based devices is the large current density requirement to drive the DW along the electron flow direction. Permalloy $\left(\mathrm{Ni}_{80} \mathrm{Fe}_{20}\right)$ has been intensively investigated due to its easy detection via anisotropic magnetoresistance (AMR) and negligible magnetostriction [19-23]. However, potential disadvantages of inplane materials include difficulty in achieving high speed, controlled DW motion and large current density requirement [24-25]. The solution lies in developing new material stacks with high magnetocrystalline anisotropy and new measurement techniques.

Out-of-plane magnetized materials have been identified due to its large magnetic anisotropy compared to in-plane materials [26-27]. Particularly, there has been a lot of research in ultrathin multilayers of $\mathrm{Co} / \mathrm{Ni}$ and $\mathrm{Co} / \mathrm{Pt}$ for $\mathrm{DW}$ dynamics [28-31]. However, ultrathin layers could be challenging to implement as regard to large current densities which may cause heat dissipation. It has been recently proposed that the domain walls in ultrathin layers can be driven at relatively low current densities via Rashba and spin Hall effect [3234]. When these effects couple with Dzyaloshinskii-Moriya interaction (DMI), DWs can attain very high speeds.

Another key challenge is to precisely control internal profile of a domain wall [3537]. From the device stand point, transitions in domain wall profile are of paramount importance where the domain wall motion is strongly influenced by artificial pinning sites. 
Because the detailed spin configuration of a domain wall can affect the nature and strength of the pinning potential [38-39].

In order to realize DW based devices, mechanisms for domain wall manipulation, generation and detection have to be well established. In this thesis, novel methods for domain wall manipulation, generation and detection are proposed and implemented.

\subsubsection{Domain wall memory}

For DW memory, the data bits are encoded in the magnetization direction of sequence of domains separated by DWs [19]. Spin polarized currents are then used to move DWs along the nanowire [40]. The spin polarized currents move the adjacent DWs along the same direction via STT, contrary to the case where magnetic field drives adjacent DWs in opposite directions [41-42]. The operation speed of the device is determined by how fast the DW moves within the nanowire while storage density depends on how closely two nanowires can be placed together. Various DW based memory devices have been proposed to replace the mechanical motion of magnetic media in HDDs [19, 43-46].

The first prototype of DW based memory device, known as racetrack memory, was

proposed by IBM $[19,47]$. It promises to attain a data storage density and manufacturing cost comparable to the conventional magnetic hard drives while achieving speed and low power consumption similar to charge based solid state memory devices. Domain walls can be stored at zero current by employing artificial pinning sites and the distance between two pinning sites determines the storage capacity of the device [48].

Fig. 1.1 shows schematic design of a DW based memory device; lateral racetrack memory. The operation of the device is similar to a solid state, non-volatile shift register 
[49]. On one side of the racetrack, the data writing can be accomplished by injecting short current pulses through a conductor placed in close proximity to the nanowire.

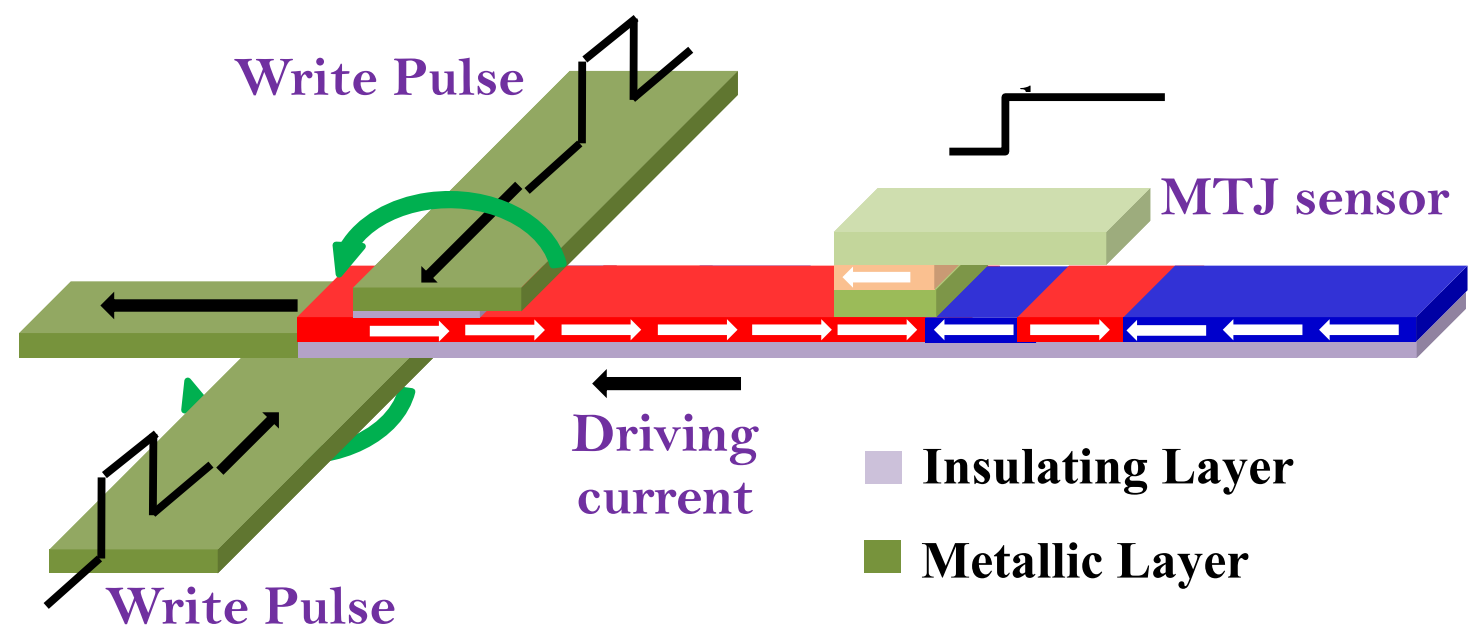

Fig. 1.1 Schematic of a DW based memory device, where bits of information are encoded as a sequence of domains. A write pulse on the left generates local Oersted field which in turn nucleates a domain wall. Pulses of highly spin polarized current moves the domain walls via spin transfer torque effect. Reading of the data is carried out by measuring tunnel magnetoresistance of a magnetic tunnel junction.

The data reading can be performed using conventional magnetic tunnel junction (MTJ) sensor attached to the other end of the racetrack. A single racetrack can store up to 100 bits of information which is 100 times more than existing random access memories (RAMs). It has been estimated that a data rate of $500 \mathrm{Mb} / \mathrm{s}$ can be achieved at a power smaller than $1 \mathrm{~mW}$ per racetrack [50-51]. To date, various versions of the racetrack memory have been proposed; DW motion in in-plane or out-of-plane magnetic materials, driven by STT or spin-orbit torque (SOT) and in mono-layer or exchange coupled stacks [52-55]. 
Further, NEC Corporation from Japan has proposed a MRAM cell with current induced DW motion in perpendicularly magnetized anisotropy materials [56]. As shown in Fig. 1.2, the device comprises of three regions; two fixed regions, whose magnetizations are aligned anti-parallel and one free region, which can be magnetized in either direction.

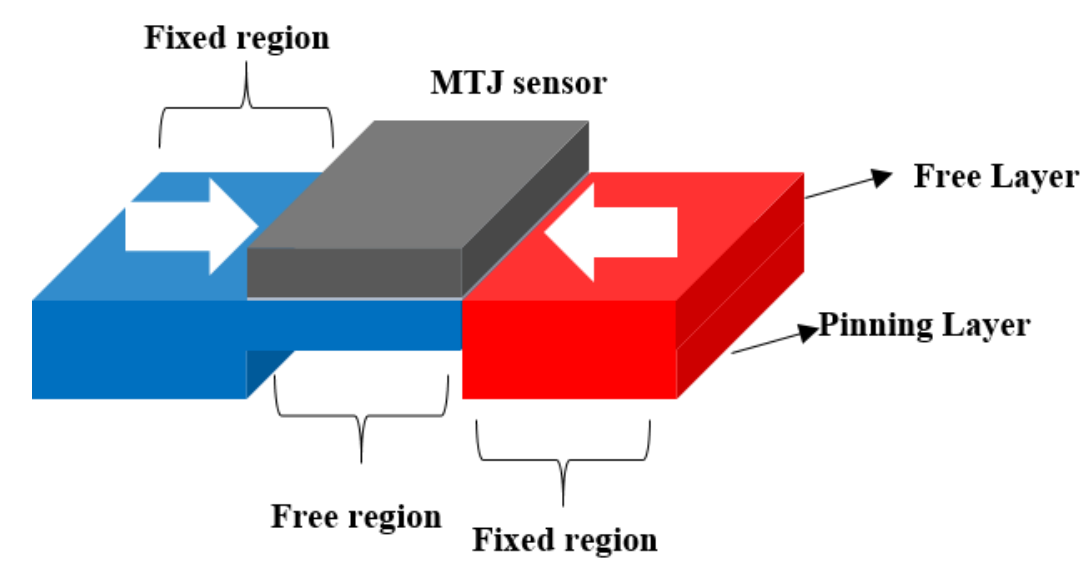

Fig. 1.2 DW motion MRAM device. It consists of two fixed regions, whose magnetization directions are always anti-parallel and one free region, where the magnetization can be directed in either direction. An MTJ sensor reads the data [56].

Since the DW always exists in the structure, the bit can be toggled between "0" and "1" by simply moving the DW across the free region. The reading of the data is performed through a MTJ sensor.

Conventionally, DWs along a nanowire are moved by applying an external magnetic field. However, the external field moves two adjacent DWs in opposite directions, so devices requiring unidirectional motion of DWs are very difficult implement field-driven scheme [57]. Recently, a new device concept has been proposed, where unidirectional motion of DWs in the presence of an external magnetic field is achieved [58]. This scheme is based on chiral DWs which exhibit asymmetry in their velocities with respect to an in- 
plane magnetic field. This device concept eliminates joule heating effect caused by high current density requirements and structural modulation which reduces threshold current density by introducing DW tension.

\subsubsection{Domain wall logic}

In addition to data storage, DW can also execute logic operations [43-44, 59]. The DW based logic devices have advantages of high scalability, non-volatility, quiescent power and fast processing speed. Allwood et al., have demonstrated the first magnetic logic device. When it propagates through these devices, DWs not only transport the information but also perform some logic operations between writing and detecting. It has been shown that the device can perform all types of logic operation which include NOT, AND, crossover and fan-out as shown in Fig. 1.3 (a). Sub-micrometer planar nanowires of in-plane magnetized materials $\left(\mathrm{Ni}_{80} \mathrm{Fe}_{20}\right)$ have been employed as conduits for $\mathrm{DW}$ propagation. The two possible logic states are assigned to two directions of in-plane magnetization. As these conduits are not simple straight nanowires, a global rotating magnetic field with a vector orients in the plane of the nanowire was used. This magnetic field allows the domain wall to cross the turns and change the direction.

The advantage of the domain wall logic over its semiconductor counterparts lies in its simple structures. For instance, in semiconductor technology, a logic AND operation includes 6 transistors while for a domain wall logic it is simple bifurcated structure which can reduce the complexity in the fabrication as well as the cost of the device. All the logic gates can be integrated into a single circuit. Fig. 1.3 (b) shows schematic of a semiconductor logic device and domain wall logic device for various operations. Further, magnetic domain wall logic device features low power consumption. 
(a)
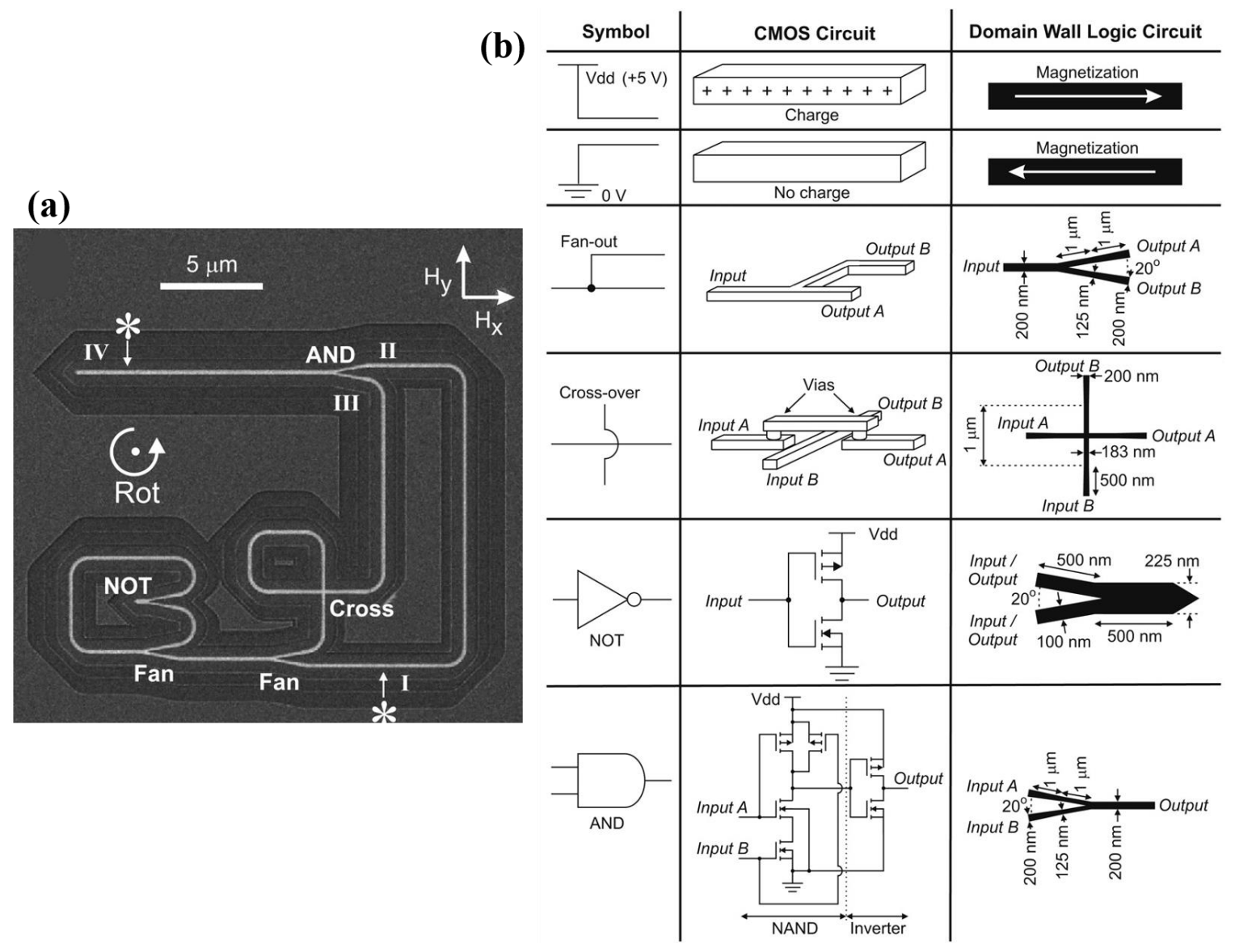

Fig. 1.3 (a) Focused ion-beam image of a domain wall based magnetic logic device, which comprises of one NOT gate, one AND gate, two fan-out junctions and one cross-over junction (b) Schematic of a semiconductor logic device and domain wall logic device. [44].

In spite of many advantages of magnetic DW logic, it poses some problems which need to be addressed. A global magnetic field affects all the components of the logic device at the same time. But, it becomes an issue when one needs the output from a particular device.

Recently, Murapaka et al. have demonstrated a magnetic logic which can perform all basic logic operations within a single device [60]. It has an advantage of non-volatility, energy efficient over the conventional semiconductor devices. Fig. 1.4 shows a schematic of reconfigurable magnetic logic device. It exploits controlled domain wall motion in an in- 
plane magnetized asymmetric branch structure. The controllability of the device is carried out via a current carrying strip line, which generates an Oersted field. The field determines the trajectory of a domain wall within the structure. By simply changing the polarity of the current, two universal logic operations can be performed. The device consists of two orthogonal nanowires connected to a half ring. The magnetization orientations of the two nanowires represent two input data bits: "input 1" and "input 2". Whereas the output bits are distinguished by the magnetization directions of individual branches of the half ring. An applied in-plane magnetic field serves as a clocking. Clocking is achieved by an alternating pulse field.

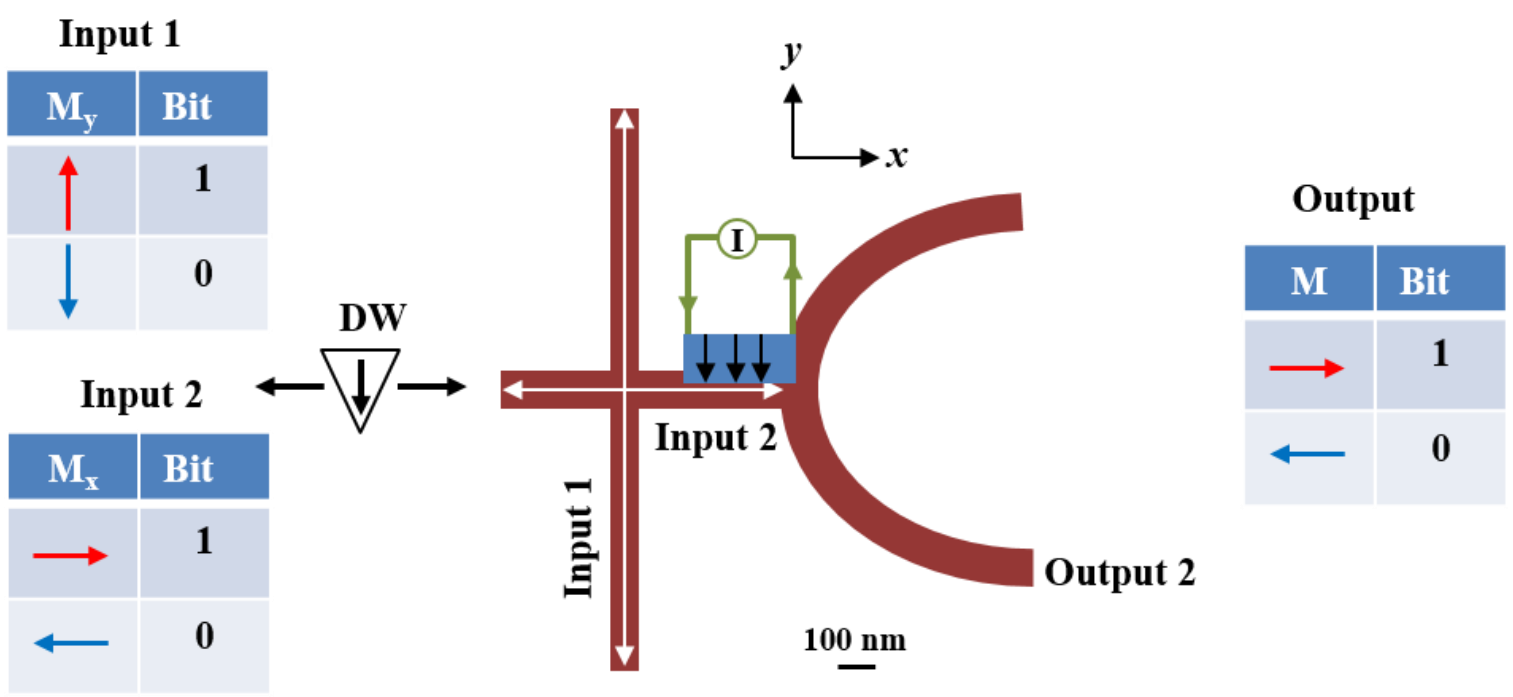

Fig. 1.4 Schematic of an all-magnetic reconfigurable magnetic logic device [60]. The magnetization directions of horizontal and vertical nanowires are considered as input 1 and input 2. The magnetization directions of upper and lower half rings represent the outputs of the device. A conduction strip line at the bifurcation acts as a gate which controls different logic operations.

As such, memory is used to store the data while logic is to compute the data. A novel DW based integrated circuit which comprises of both DW based memory and logic 
devices can be realized for ultra-high speed, low cost, and non-volatility. However, there is need for better understanding the DW properties and dynamics in ferromagnetic nanostructures.

\subsection{A brief introduction to ferromagnetism}

In order to understand the origin of domains and DWs in ferromagnetic materials, it is imperative to start with the basics of ferromagnetism. The first theoretical prediction of the origin of ferromagnetism was provided by Weiss based on molecular field theory [61]. Wiess postulated that the magnetic interactions among magnetic moments is equivalent to an internal interaction field which aligns the magnetic moments in specific direction. Weiss derived the temperature dependent spontaneous magnetization of a ferromagnetic material. This phenomenon is known as Curie-Weiss law which states that an ordering of spins in a ferromagnetic material is preserved up to the Curie temperature $T_{C}$, above which the spins orient randomly due to thermal fluctuations. The Weiss molecular field for an average

ferromagnetic material was calculated to be in the order of $10^{9} \mathrm{~A} / \mathrm{m}$, while an external field of few tens of $\mathrm{A} / \mathrm{m}$ has been shown to switch the magnetization. To explain this discrepancy, Weiss proposed the concept of magnetic domains. Inside an individual domain, spontaneous alignment of magnetic moments occurs while the orientation of each domain can be different. The net magnetic moment of a ferromagnet is the average magnetization of all the domains. The Weiss theory is based on classical thermodynamics and it forms a basis for understanding ferromagnetism. However, the origin of the molecular field theory cannot be fully accounted by classical physics.

A quantum mechanical exchange phenomenon has been put forward to fully address the origin of molecular field. Heisenberg proposed that some materials exhibit finite 
magnetic moment in the absence of external magnetic field due to a strong exchange interaction between neighboring atoms [62]. The Heisenberg exchange model differs from the Weiss model in that it describes the interaction between only adjacent atoms in a ferromagnetic system rather than applying an averaged mean field approximation. Heisenberg succeeded in demonstrating that the molecular field is equivalent to the exchange field, which tend to align adjacent spins parallel to each other. As such, the exchange interaction is an electrostatic interaction. Heisenberg exchange phenomenon provides the basic understanding of ferromagnetism. For mesoscopic objects, magnetic properties cannot be described using either Weiss or Heisenberg model. So, micromagnetics becomes an appropriate theoretical approach to address these issues [63].

Micromagnetics focuses on the coupling between magnetic moments at submicron length scale. Ferromagnetic systems are considered as classical continuum with boundary conditions, so that the magnetization can be treated as a continuous function, $M(r, t)$. The magnetization $M(r, t)$ is continuous function of space and time. Before focusing on the magnetization switching, it is crucial to understand various interactions and related energy contributions.

\subsection{Free energy of a ferromagnetic system}

The free energy of a ferromagnetic system under the influence of an external magnetic field or current comprises of four main energy terms:

$$
E_{\text {total }}=E_{\text {exchange }}+E_{\text {zeeman }}+E_{\text {magnetocrystalline }}+E_{\text {magnetostatic }}
$$




\subsubsection{Exchange energy}

An exchange interaction between two neighbouring atoms in a ferromagnetic material arises from a combined effect of Pauli's exclusion principle and Coulomb interaction. The exchange interaction acts to align adjacent atoms by minimizing the exchange energy of the system. The net exchange energy of the system can be defined as the summation of the interactions between all atoms. It can be defined as

$$
E_{\text {Exchange }}=-2 J_{e x} \sum S_{i} \cdot S_{j}=-2 J_{e x} S^{2} \sum \cos \phi_{i, j},
$$

where $J_{e x}$ is the exchange integral, $S_{i}$ and $S_{j}$ are the spin angular momenta of two neighbouring atoms $i, j$ respectively and $\phi_{i, j}$ is angle between two spins. Fig. 1.5 (a) shows the schematic of exchange interaction between different spins. For a ferromagnetic material, the exchange integral is positive, $J_{e x}>0$ and hence the spins tend to align parallel to each other. Whereas in antiferromagnetic material, $J_{e x}<0$ resulting in anti-parallel orientation of spins. The exchange stiffness constant $(A)$ can be related to $J_{e x}$ as

$$
A=\frac{J_{e x}}{a^{2}},
$$

where $a$ is the lattice constant. The exchange stiffness constant $(A)$ gives a measure of the energy, which is associated with misalignment of neighbouring spins. As shown in Fig. 1.5 (b), the misalignment of spins would lead to an exchange energy penalty. The characteristic exchange length of a ferromagnetic material represents a spatial scale below which the atomic moments must remain aligned due to exchange forces.

The exchange length is given by the following equation

$$
L=\sqrt{\frac{2 A}{\mu_{0} M_{s}^{2}}},
$$


where $M_{s}$ is the saturation magnetization and $A$ is the exchange stiffness constant.

(a)

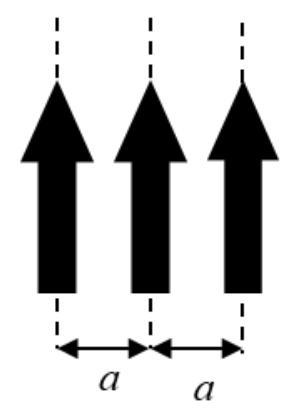

(b)

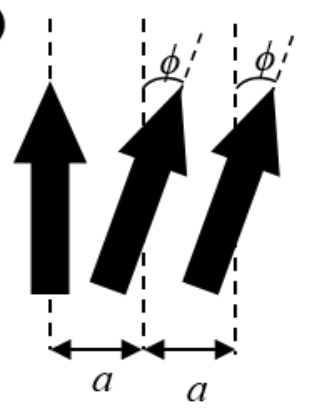

Fig. 1.5 (a) Schematic of the ferromagnetic exchange interaction between neighbouring spins. (b) A misalignment of spins $\phi$ results in the increase of the exchange energy.

For permalloy $\left(\mathrm{Ni}_{80} \mathrm{Fe}_{20}\right)$, typical values of $M_{s}$ and $A$ are $8 \times 10^{5} \mathrm{~A} / \mathrm{m}, 1.3 \times 10^{-11}$ $\mathrm{J} / \mathrm{m}$ respectively and the exchange length is estimated to be $\sim 5 \mathrm{~nm}$. Whereas for perpendicularly magnetized materials (Co/Ni), typical values of $M_{s}$ and $A$ are $3 \times 10^{5} \mathrm{~A} / \mathrm{m}$, $1 \times 10^{-11} \mathrm{~J} / \mathrm{m}$ respectively and corresponding exchange length $(L)$ is $13 \mathrm{~nm}$ [64].

\subsubsection{Zeeman energy}

The Zeeman energy is associated with an interaction between magnetic moments and external magnetic field $\left(H_{\text {ext }}\right)$. When the magnetization of the system is aligned along the direction of magnetic field, this energy gets minimized. The energy can be expressed as

$$
E_{\text {Zeeman }}=-\mu_{0} \vec{H}_{e x t} \cdot \vec{M}
$$

where $\vec{H}_{e x t}$ is the externally applied magnetic field, $\vec{M}$ is the magnetization and $\mu_{0}$ is the magnetic permeability of vacuum. 


\subsubsection{Magneto crystalline anisotropy energy}

In a ferromagnetic crystal, the magnetization prefers to align along a specific crystallographic axis, which is often referred to as "easy" axis of magnetization. For instance, in a hcp crystalline structure, the easy axis would be along the $c$ axis of the hexagonal cell. The directions which are unfavorable for the magnetization to align are termed as "hard" axis of magnetization. The energy cost required to align the magnetization along the hard axis is known as magneto crystalline anisotropy energy.

In a system with simple uniaxial anisotropy, the anisotropy energy can be expressed as

$$
E_{\text {magnetocrystalline }}=K_{u} \sin ^{2} \theta
$$

where $\theta$ is the angle between the anisotropy axis and the magnetization direction, $K_{u}$ is the uniaxial anisotropy constant. Higher order terms, like $K_{u} \sin ^{4} \theta$ and $K_{u} \sin ^{6} \theta$, have been found to be insignificant.

\subsubsection{Magnetostatic energy}

Magnetostatic energy corresponds to an interaction between the magnetization of a material and its demagnetizing field, $H_{d m}$. The magnetostatic energy can be expressed as

$$
E_{\text {magnetostatic }}=\frac{1}{2} \mu_{0} \vec{H}_{d m} \bullet \vec{M} d V
$$

where $\vec{H}_{d m}$ is the demagnetizing field and $d V$ is the volume of the space. $\vec{H}_{d m}$ scales linearly with the magnetization and depends on the geometry of the structure.

$$
\vec{H}_{d m}=-N \cdot \vec{M}
$$

where $\mathrm{N}$ is the demagnetizing tensor. The demagnetizing coefficients along $x, y$ and $z$-axis are given as $N_{x}, N_{y}$ and $N_{z}$ such that $N_{x}+N_{y}+N_{z}=1$. The magnetostatic energy is equivalent 
to an electrostatic energy which exists between two electric charges. The strength of the magnetostatic energy is small as compared to the exchange interaction. However, the magnetostatic energy is very important as it influences the spatial distribution of the magnetization. Energy minimization from a competition between magnetostatic and exchange energy leads to the formation of domain structure.

\subsubsection{Dzyaloshinskii-Moriya interaction}

As described in sec. 1.3.1, an exchange interaction acts to align adjacent spins parallel to each other, causing exchange energy minimization [65].

The exchange energy can be written in terms of Hamiltonian as

$$
H_{\text {exchange }}=-\sum_{i, j} J_{i, j}\left[S_{i} \bullet S_{j}\right]
$$

where $J_{i j}$ is the exchange integral and $S_{i}$ and $S_{j}$ are the spin angular momenta of neighbouring spins.

However, in some materials with broken inversion symmetry, an additional interaction known as the Dzyaloshinskii-Moriya interaction (DMI) can be dominant and it introduces chiral magnetic textures [66-67]. DMI is known to originate from the strong spin-orbit coupling. The corresponding Hamiltonian can be expressed as

$$
H_{D M I}=-\sum_{i, j} D_{i, j}\left[S_{i} \times S_{j}\right]
$$

where $D_{i, j}$ is the DMI tensor and $S_{i}, S_{j}$ are the spin angular momenta of atoms $i, j$. Unlike direct exchange interaction, which favors parallel spin orientation of neighbouring spins, the DMI instead prefers perpendicular orientation. Therefore, the DMI and direct exchange interaction compete, resulting in the formation of chiral magnetic textures. 


\subsection{Magnetic domain and domain walls}

In order to reduce the total free energy of the system, a ferromagnetic material forms domain structure. Fig. 1.6 depicts schematic of domain formation due to the minimization of magnetostatic static energy. The minimization corresponds to a rotation of magnetic dipoles within the sample such that the magnetic dipoles form a minimum of free poles on the surface. As a result, the material breaks into different magnetic domains oriented in various directions. If the demagnetizing energy is the only contributing to the domain formation, the material would break itself into smaller and smaller magnetic domains, resulting in zero net magnetization. However, the final domain structure minimizes the total energy by reducing the magnetostatic energy at the cost of exchange energy.

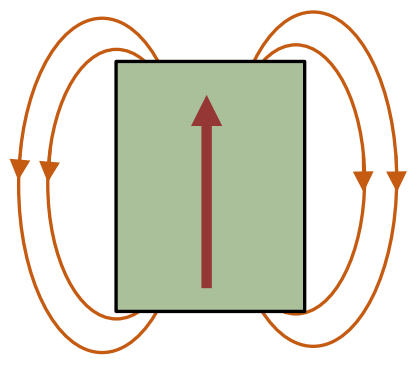

(a)

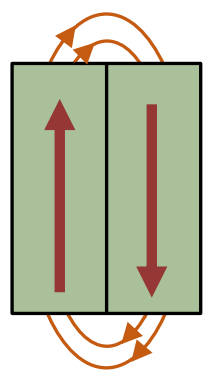

(b)

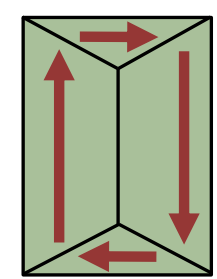

(c)

Fig. 1.6 Schematic of structure (a) with higher magnetostatic energy, (b) with magnetostatic energy become halved, (c) magnetic flux closure with almost reduced magnetostatic energy.

The competition between these two energies would lead to the formation of magnetic domains, which are separated by domain walls (DWs). A DW is a region over which the magnetization of one domain changes to the other.

The width of the DW can be expressed as 


$$
\Delta=\sqrt{\frac{A}{K}},
$$

where $A$ is exchange stiffness constant, $K$ is anisotropy constant.

The exchange energy $(A)$ favors DWs with larger width, so that the neighbouring magnetic moments are separated by small angles. However, the anisotropy energy minimizes the number of magnetic moments within the DW, which results in thin wall.

\subsection{Bloch and Néel domain walls}

Depending on the direction of rotation, domain walls can be broadly categorized as Bloch and Néel configurations [68]. Fig. 1.7 shows schematic of a Bloch and Néel DWs. When magnetic moments within a wall rotates in the plane containing the magnetizations of the two domains, Néel walls are formed [69-70].

\section{Bloch}

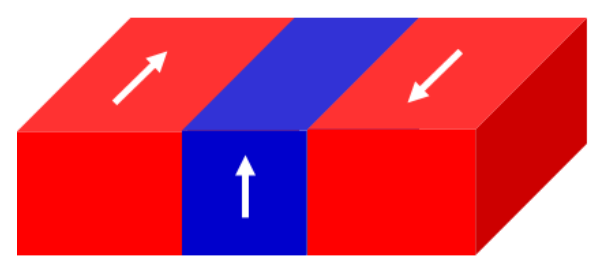

(a)
Néel

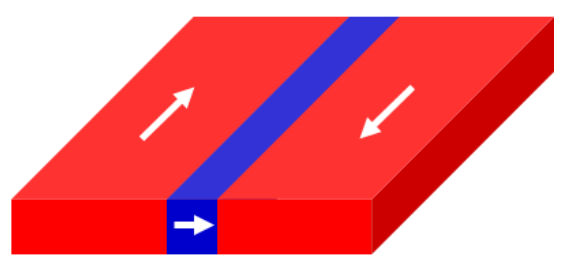

(b)

Fig. 1.7 Schematic illustrations of (a) Bloch and (b) Néel DWs in ferromagnetic systems with in-plane magnetic anisotropy. For a Bloch DW, magnetization of the wall rotates perpendicular to the plane and for Néel wall, the magnetization lies in-plane.

Néel walls are preferred in ferromagnetic materials with in-plane magnetic anisotropy, conversely, when the magnetization of the wall rotates perpendicular to the 
plane, Bloch walls are formed [71]. By minimizing the magnetostatic energy, the formation of Bloch or Néel is determined by $\frac{w}{\Delta}$, where $w$ is the width of the strip and $\Delta$ represents the width of the domain wall [72]. When this ratio is high, Bloch wall is preferred while for smaller ratio Néel is favored.

\subsection{Transverse and vortex domain walls}

In nanowires with negligible magnetocrystalline anisotropy, the competition between magnetostatic and exchange energy lead to two types of DWs: vortex and transverse [69]. In a transverse DW, the magnetization at the center of wall orients along the transverse direction $(y)$ of the NW due to larger magnetostatic interaction. As shown in Fig. 1.8, the nanowire geometry allows the wall distortion along the $y$-axis. A vortex wall forms a flux closure.

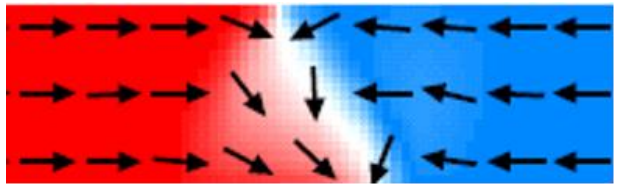

Transverse DW
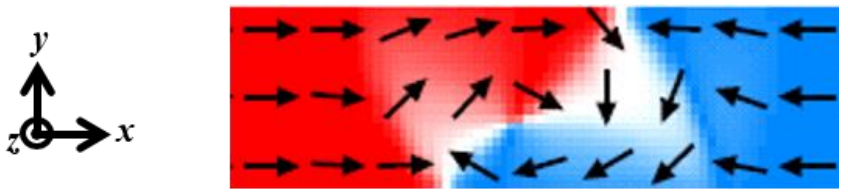

Vortex DW

Fig. 1.8 Schematic of spin configuration of (a) transverse and (b) vortex domain walls. For a transverse $D W$, the magnetization at the centre of the wall orients along the transverse direction of the nanowire while a vortex wall forms a flux closure.

The moments within the wall rotate clockwise or anti-clockwise around the vortex core, which determines the wall orientation. The moments at the vortex core point either upward or downward. The type of DW formation depends on the geometry and material 
properties. The phase diagram for a stable DW configuration with respect to geometry of the nanostructure was first given by M. Donahue [73]. Further, Fig. 1.9 shows the phase diagram for a stable domain wall configuration with strip geometry.

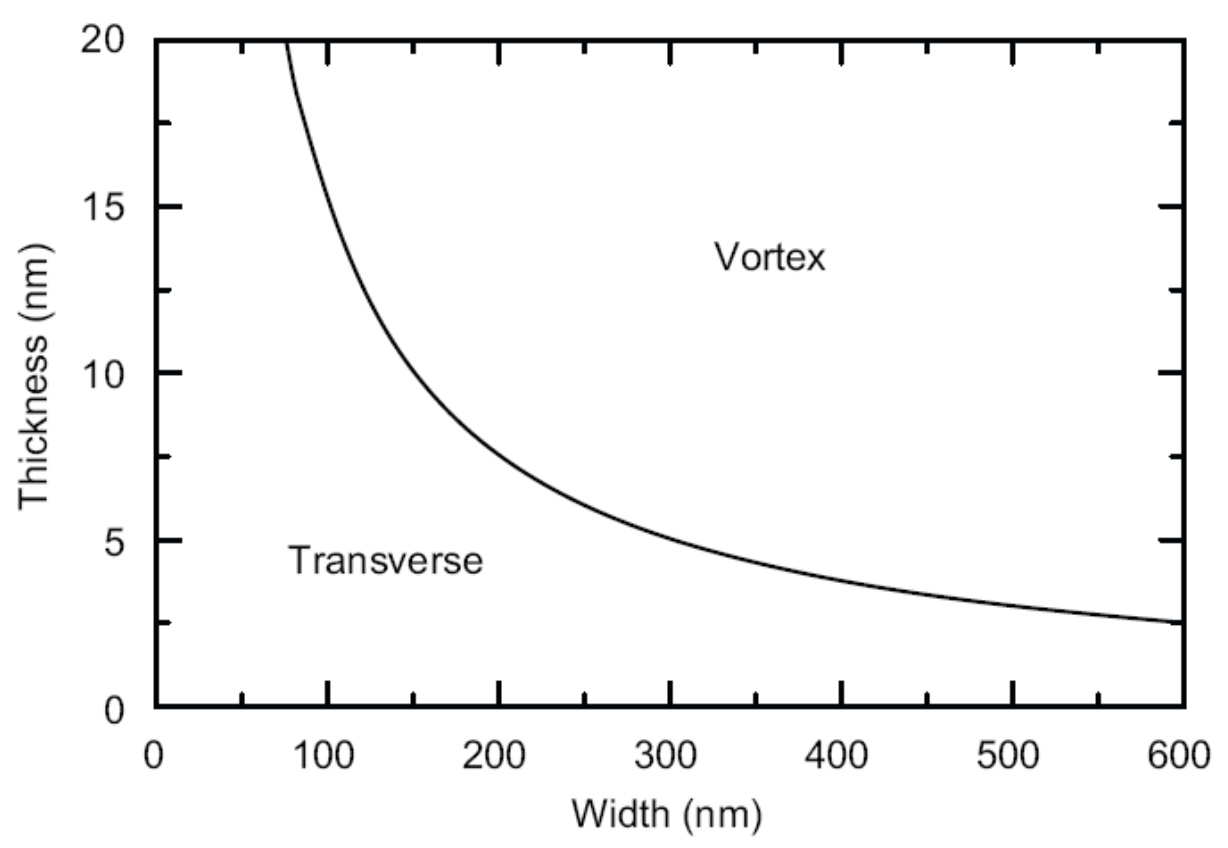

Fig. 1.9 Phase diagram of the stability of transverse and vortex DWs in permalloy nanowire as a function of its width and thickness [74].

\subsection{Magnetic charge possessed by a transverse domain wall}

Based on the orientation of magnetization within the domain, transverse walls are differentiated as head-to-head (HH) or tail-to-tail (TT) DW. Fig. 1.10 shows the schematic of HH and TT DWs. HH DW is characterized by a positive charge while TT DW is characterized by a negative magnetic charge. The magnetic charge of a transverse domain wall can be determined by taking the divergence of its magnetization, 


$$
Q=-\nabla \cdot \vec{M}
$$

where $Q$ is the magnetic charge and $M$ is the magnetization.
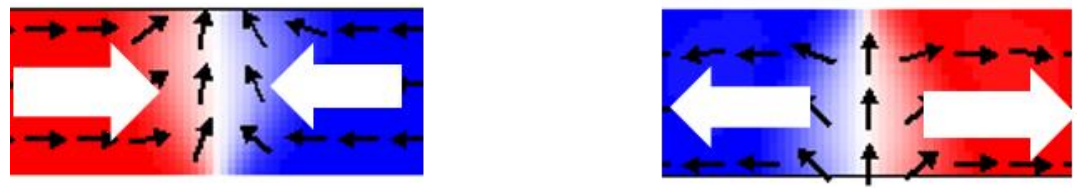

Fig. 1.10 Schematic of the HH and TT configurations of a transverse DW. For a HH DW the magnetizations of the two domains point towards each other while for a TT DW the magnetizations point away from each other.

A transverse DW is characterized by a triangular shape [75]. Fig. 1.11 shows the calculated magnetic charge at the base and apex of the triangular shape.
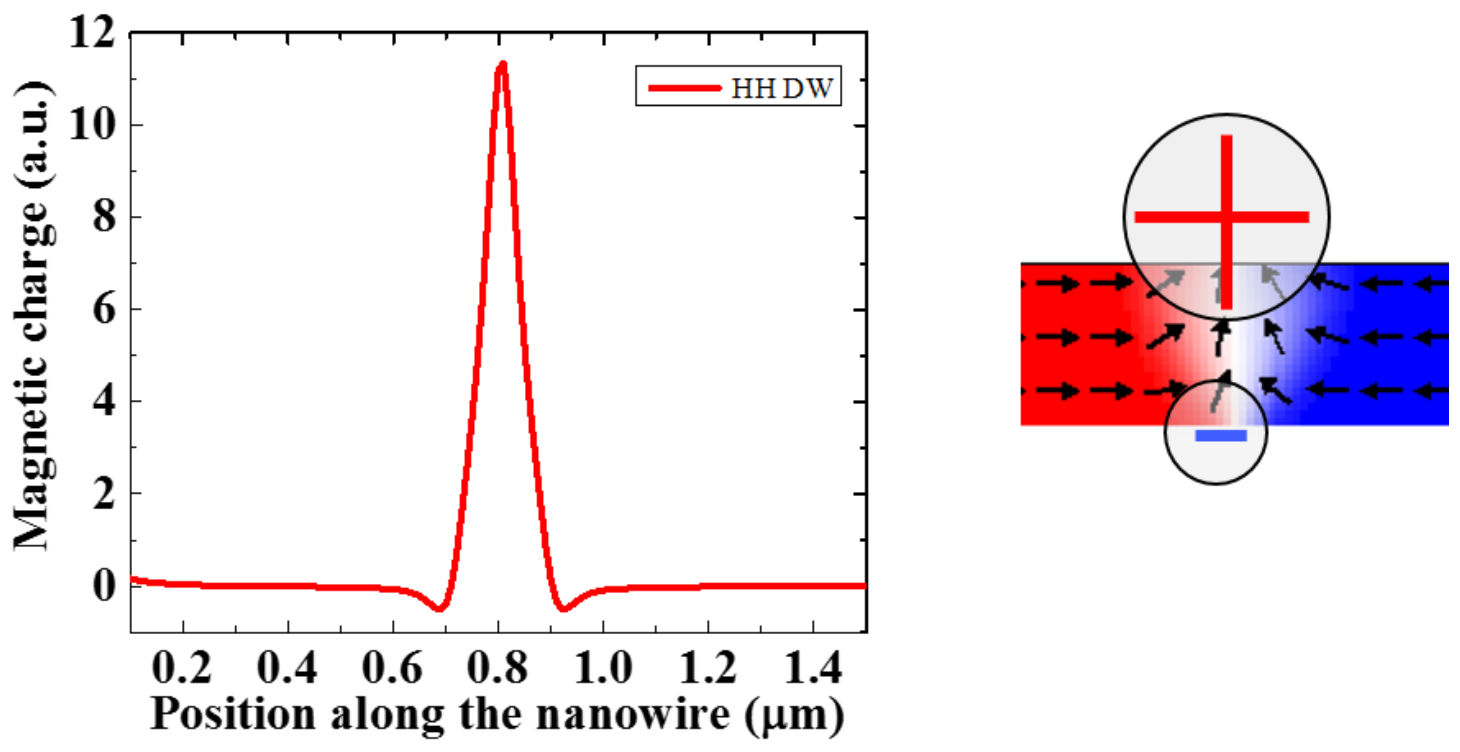

Fig. 1.11 Magnetic charge possessed by a HH transverse DW. Magnetic volume pole of a DW along the transverse direction. 
For a HHDW, the base of the triangle contains positive charge while the apex has negative magnetic charge. The magnetic pole volume measured as a function of nanowire length shows that the magnitude of the positive charge is large compared to that of negative charge. The magnetic volume pole is similar to a magnetic charge which is magnetic analogue of an electrical charge. As such, a HHDW possesses positive magnetic charge. Similarly, a TTDW possesses negative magnetic charge. The magnetic charge of a transverse DW plays significant role in the DW interaction [76-78].

Besides the magnetic charge, a transverse DW has another degree of freedom, often called as chirality. The chirality of a DW is represented by the transverse direction of the magnetization within the DW. Fig. 1.12 shows the schematic of Up chirality and Down chirality DWs. When the transverse magnetization points along the $+y$ direction, the DW is said to have "Up" chirality while the magnetization points along $-y$ axis, it is called "Down" chirality.
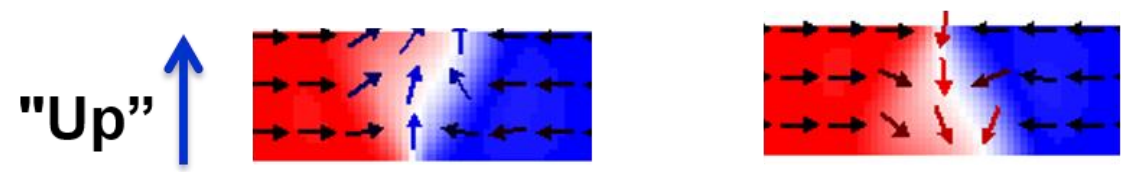

\section{"Down"}

Fig. 1.12 The transverse profile of a DW can either orient along $+y$ or $-y$. When it orients along $+y$, the DW has Up chirality and along $-y$, the DW has Down chirality.

\subsection{Topological defects}

A transverse DW can be described as a composite object of elementary topological bulk and edge defects [79-81]. The spatial arrangement of the topological defects determines the chirality of a transverse DW. The topological defects are characterized by 
winding numbers. The winding number $(n)$ for an edge defect can be defined as the line integral along the edge of the nanostructure [81] and it is given by

$$
n=-\frac{1}{2 \pi} \int_{\partial \Omega} \nabla\left(\theta-\theta_{\tau}\right) \cdot d r
$$

where edge defect is characterized by either $\pm 1 / 2$. The winding number (n) for a bulk topological defect can be defined as [79],

$$
n=\frac{1}{2 \pi} \oint_{\partial \Omega} \nabla \theta \cdot d r
$$

where $n$ takes integer values $( \pm 1)$ and $\Omega$, is a two dimensional region $r(r, \phi)$, encompasses a bulk defect. According to the theory of topological defects, the total winding number of any ferromagnetic system is conserved [79].

$$
n_{\text {total }}=\sum_{i}^{\text {bulk }} n_{i}+\sum_{j}^{\text {edge }} n_{j}=1-g
$$

where $g$ represents the number of holes present in a ferromagnetic system. The sum of the winding numbers of topological defects with in a single DW is zero. It implies that the presence of a DW in a ferromagnetic system should not change the total winding number $\left(n_{\text {total }}\right)$. Fig. 1.13 shows schematic of topological edge and bulk defects. 
(a)

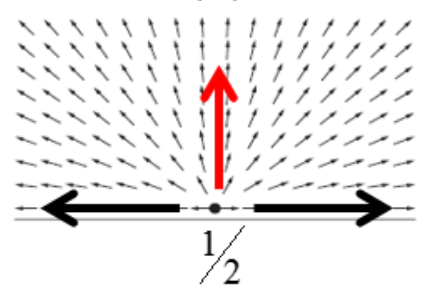

(b)

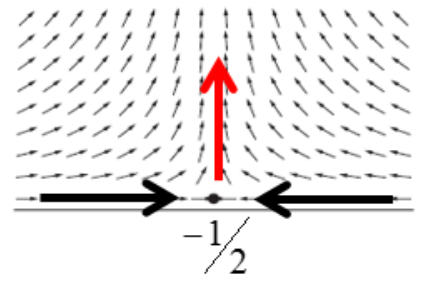

(c)

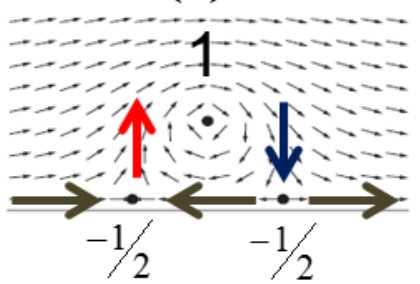

Fig. 1.13 Topological edge defect with winding numbers (a) $n=1 / 2$, (b) $n=-1 / 2$ and (c) vortex with $n=+1$. The sum of the winding numbers of topological defects is always zero.

For example, a vortex DW is comprises of a bulk defect $(n=+1)$ at its center and two edge defects $(n=-1 / 2)$, resulting in $n_{\text {total }}^{D W}=0$. As shown in Fig. 1.14, a traverse DW consists of two edge defects $n=-1 / 2$ and $1 / 2$, which again results in results in $n_{\text {total }}^{D W}=0$. A transverse DW exists in four possible spin configurations with different winding numbers.
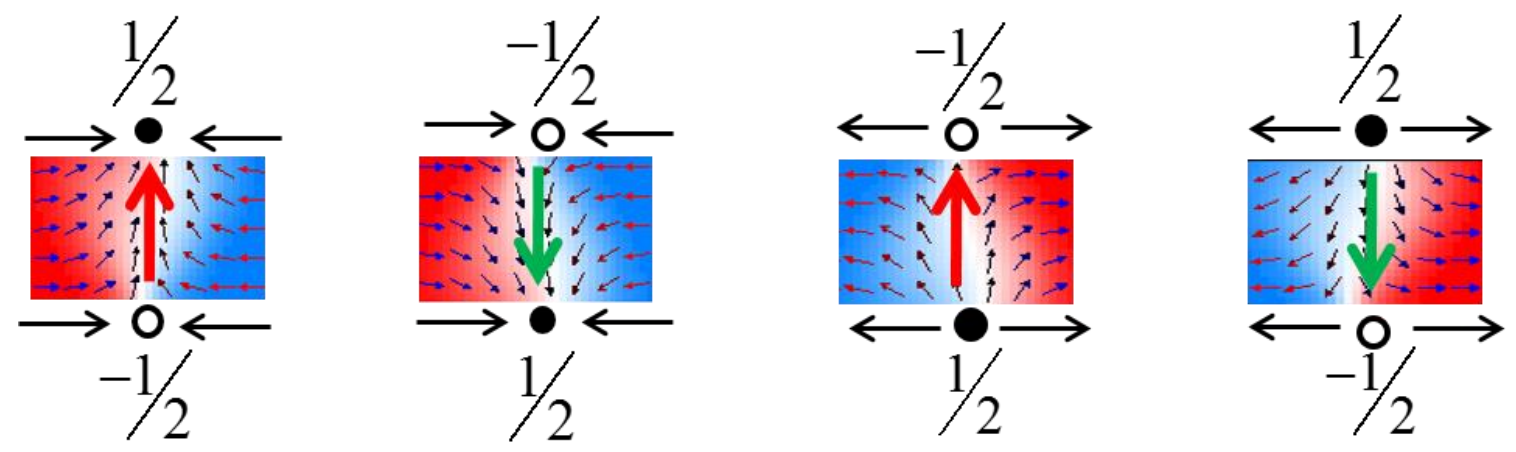

Fig. 1.14 Four possible configurations of a transverse domain wall with different winding numbers.

The conservation of topological charge has important implications on the magnetization dynamics in a ferromagnetic system. DWs with same winding numbers undergoes the same depinning mechanisms at artificial defect. Irrespective of the magnetic charge of a transverse DW, when two DWs carry same winding number, it results in the 
formation of bound state. When DWs carry opposite winding number, they mutually annihilate each other [82-83]. In a coupled nanowire system, strength of the coupling between two DWs is strongly influenced by the edge defects of two interacting DWs [84].

\subsection{Domain walls in in uniaxial systems}

In thin films with strong uniaxial anisotropy, the easy axis of magnetization will be out-of-plane. The widths of the DW in out-of-plane magnetized thin films is smaller than the width of the nanowire. Thus, the magnetization rotating perpendicular to the DW width would cost lesser dipolar energy compared to the magnetization rotation along the DW. The preferred DW configuration will be Bloch. Néel DW structures are favourable in narrow nanowires. Fig. 1.15 shows the schematic of Néel and Bloch configurations in a thin film with strong uniaxial anisotropy. The transition from Bloch wall to Néel $\mathrm{DW}$ in $\mathrm{Co} / \mathrm{Ni}$ multilayers typically occurs at $\sim 60 \mathrm{~nm}$ NW width [85].

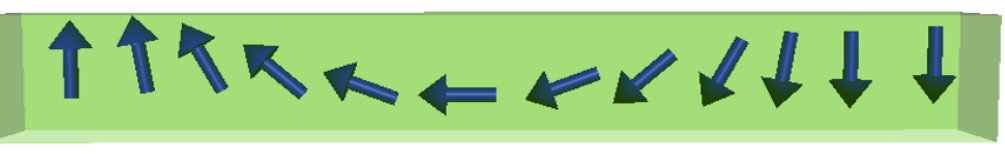

(a)

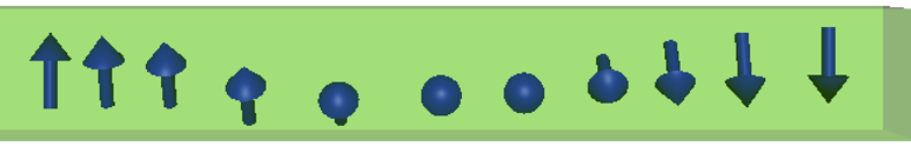

(b)

Fig. 1.15 Schematic of Néel (a) and Bloch (b) configurations in out-of-plane magnetized materials.

In out-of-plane materials with structural inversion asymmetry, Néel DWs are favored [86-88]. An additional energy term due to Dzyaloshinskii-Moriya interaction dominates over magnetostatic energy and stabilizes Néel DWs. Additionally, these Néel DWs have a fixed chirality [89]; left-handed or right-handed chirality as shown in Fig. 1.16. 


\section{$\uparrow \uparrow イ \leftarrow ル \Downarrow \downarrow$}

Left-handed Neel wall

\section{$\uparrow \uparrow ネ \rightarrow ロ \downarrow \downarrow$}

right-handed Neel wall

Fig. 1.16 DMI induced chiralities of Néel domain walls. The magnetization of the domain wall at the centre is saturated along either $+x$ or $-x$ direction.

\subsection{Magnetization dynamics: Landau-Lifshitz-Gilbert equation}

The temporal evolution of magnetization under the influence of field or current can be described by Landau Lifshitz Gilbert (LLG) equation. This equation was proposed by Landau Lifshitz and then modified by Gilbert. When an external magnetic field is applied to a ferromagnetic system, the magnetization would precess around the magnetic field axis due to a torque given by [89],

$$
\frac{\partial \vec{M}}{\partial t}=-\gamma \vec{M} \times \vec{H}_{e f f}
$$

where $\gamma$ is the gyromagnetic ratio and $H_{\text {eff }}$ is the effective field arises from the contributions of exchange field, magneto-crystalline anisotropy field and external field (Zeeman field) etc. This equation describes the continuous precessional motion of a magnetization vector $\vec{M}$, where the dissipation mechanism is not considered. Hence, the equation has been modified to include the damping term. The modified LLG equation for the net magnetization can be written as sum of precessional term and dissipation term and is given by [90],

$$
\frac{\partial \vec{M}}{\partial t}=-\gamma \vec{M} \times \vec{H}_{e f f}+\alpha\left(\vec{M} \times \frac{\partial \vec{M}}{\partial t}\right),
$$


where $\alpha$ represents the Gilbert damping constant, $\alpha>0$. Fig. 1.17 shows schematic of the magnetization precession with and without damping. The damping process is associated with an energy transfer from the ferromagnetic system to the other degrees of freedom such as lattice through spin-orbit coupling.

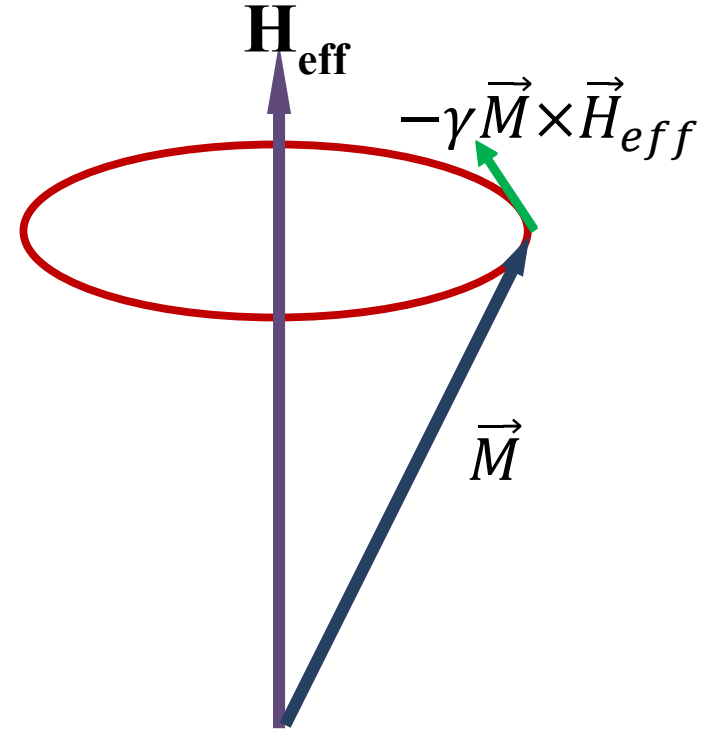

(a)

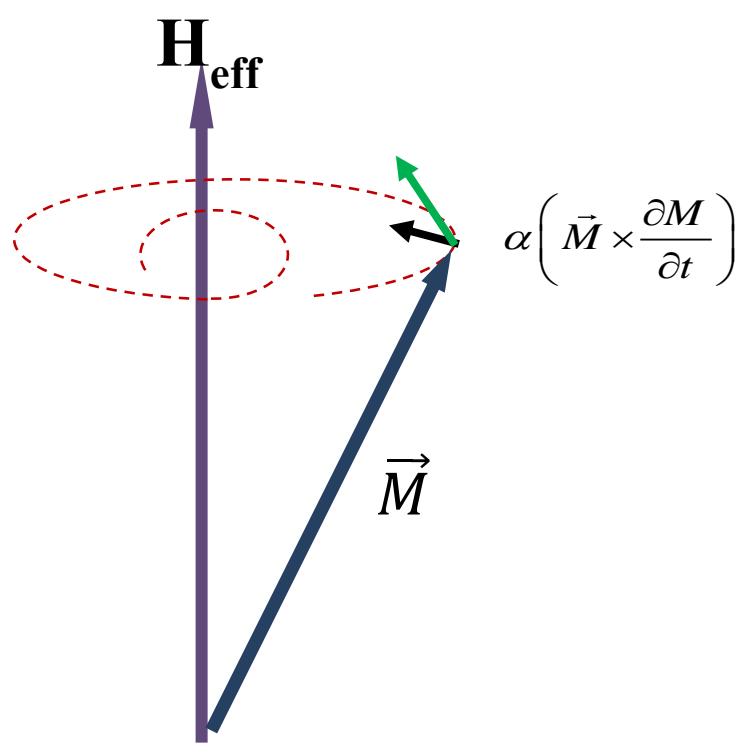

(b)

Fig. 1.17 Schematic of the magnetization dynamics in the presence of an external magnetic field. (a) Precession of the magnetization without damping mechanism. (b) Precession in the presence of damping. The magnetization relaxes to an equilibrium state.

\subsubsection{Field induced domain wall motion}

The field induced domain wall motion is due to the Zeeman energy associated with an interaction between the magnetic moments of a DW and external magnetic field. The field induced DW motion is bidirectional; magnetic domains aligned along the field direction are energetically favored and expand with increasing field. Magnetic domains aligned anti-parallel with the magnetic field shrink as the external field is increased. Fig. 
1.18 shows the schematic of the field induced DW motion in in-plane and out-of-plane magnetized thin films.

For in-plane magnetized material, we assume that the width of the nanowire is narrow such that transverse DW is the stable DW configuration. When an external in-plane field is applied to drive the transverse DW, the resulting torque, $-\gamma\left(\vec{M} \times \vec{H}_{e x t}\right)$, initially cants the DW magnetic moments out-of-plane of the thin film as shown in the schematic of the Fig. 1.18 (a).

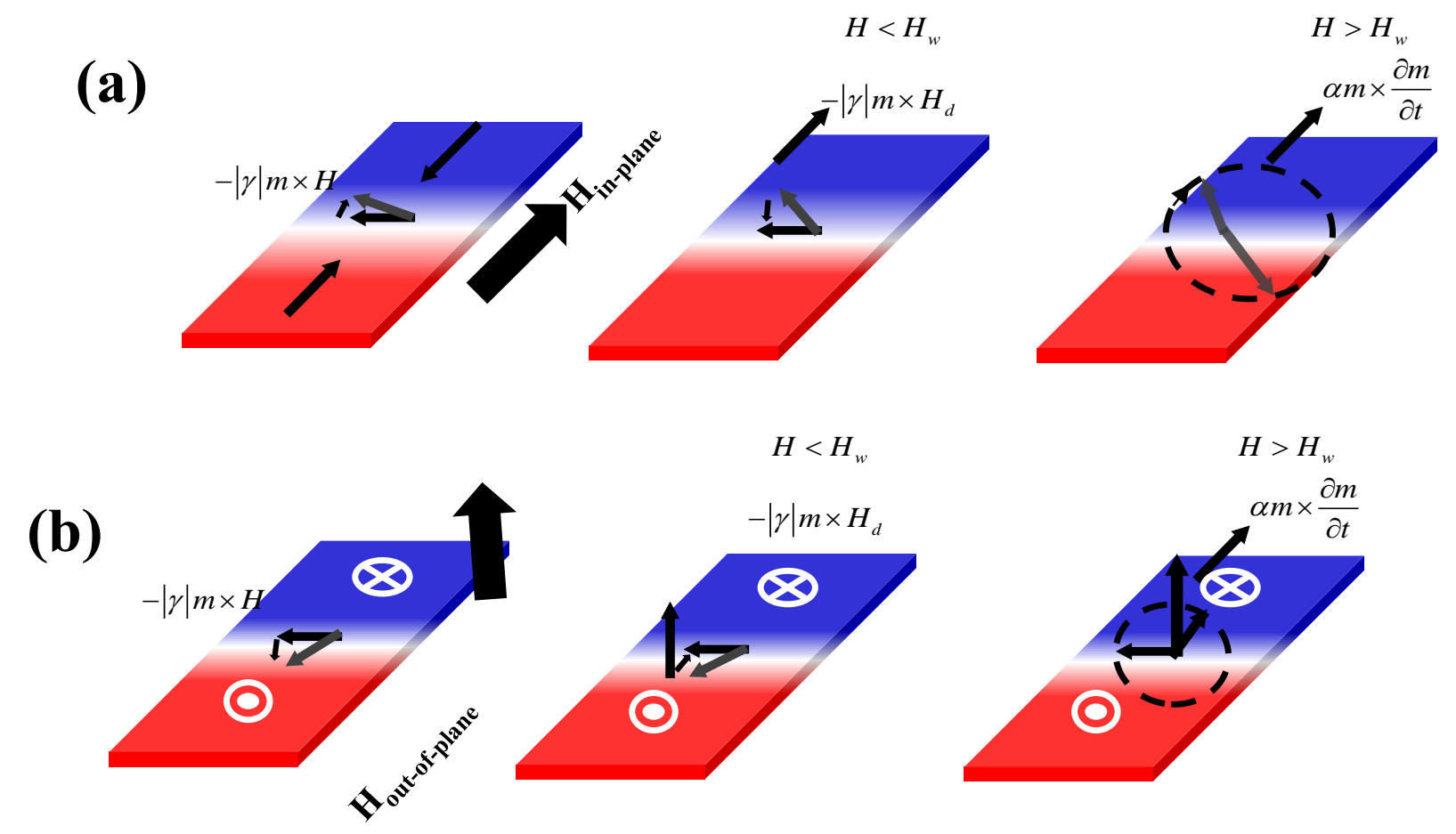

Fig. 1.18 Field induced domain wall dynamics in (a) in-plane magnetized thing film and (b) out-of-plane magnetized thin film.

The out-of-plane component of the DW moment then develops free magnetic pole on the surface. The free magnetic poles generate an additional torque, $-\gamma\left(\vec{M} \times \vec{H}_{d}\right)$ and the torque drives the DW forwards. In this field regime, known as flow regime, the velocity of 
the DW scales linearly with applied magnetic field, $v=\mu H$, where $\mu$ is the mobility of the DW. However, for larger applied magnetic fields, the DW moments no longer maintains the constant canting angle and instead the moments start to precessing around the nanowire axis. In this field regime, the DW motion is governed by a damping torque. The damping torque moves the DW less efficiently as $v=\alpha^{2} \mu H$. The non-linear behaviour of the domain wall speed is due to the change in the dynamic mode of DW from flow regime to a preccesional motion and is referred to as the Walker breakdown (WB). The critical field at which dynamic mode changes from flow regime to precessional motion is called the walker field $\left(\mathrm{H}_{\mathrm{W}}\right)[91]$.

The DW motion in out-of-plane materials can also be understood by the same principle as in-plane material. As shown in Fig. 1.18 (b), the applied magnetic field cants the DW moments in-plane, creating a Néel-like configuration. The resultant moments generate a demagnetizing field. For field strength smaller than the Walker field $H_{e x t}<H_{W}$, the resulting torque from the demagnetizing fields, $-\gamma\left(\vec{M} \times \vec{H}_{d}\right)$, drive the DW forward.

For $H_{e x t}>H_{W}$, the damping torque, $\alpha\left(\vec{M} \times \frac{\partial \vec{M}}{\partial t}\right)$, induces a similar DW dynamics as described for the in-plane films. Unlike in-plane films, the potential barrier for inducing precessional motion is significantly lower in perpendicularly magnetized materials, resulting in lower value of DW speed. This is due to the fact that the DW energetically prefer to stay in Bloch configuration.

\subsubsection{Current induced domain wall motion}

The current induced domain wall motion is unidirectional; irrespective of the magnetic domains configuration, all DWs move along same direction. The current induced 
domain wall motion is due to the interaction between the spins of conduction electrons and the local magnetization; spin torque effect (STT). Berger has proposed the idea of STT and later proved it by a series of experiments [92-94]. The first experimental studies of STT demonstrates that the current could displace DWs in permalloy $\left(\mathrm{Ni}_{80} \mathrm{Fe}_{20}\right)$ [21, 95-96]. The temporal evolution of magnetic moments in this case can be understood from the LLG equation by incorporating additional adiabatic and non-adiabatic terms,

$$
\frac{\partial \vec{M}}{\partial t}=-\gamma \vec{M} \times \vec{H}_{e f f}+\alpha\left(\vec{M} \times \frac{\partial \vec{M}}{\partial t}\right)-(u . \nabla) M-\beta M \times[-(u . \nabla) M]
$$

where the third and fourth terms represent adiabatic and non-adiabatic torques respectively and $\beta$ parametrizes the non-adiabatic STT. The spin drift velocity $(u)$, is given by

$$
u=\frac{g \mu_{B} P j_{e}}{2|e| M_{s} \partial t},
$$

where $P$ is the spin polarization of the current, $e$ is the charge of the electron and $j_{e}$ is the current density. The STT effect has been explained by the principle of conservation of total angular momentum. Conduction electrons in a ferromagnetic material are spin polarized due to spin dependent scattering. When the spin polarized electron flows across a DW, the electron's spin is flipped as it follows the spatial varying magnetization. It results in the change of spin angular momentum of electron which in turn changes the spin angular momentum of the DW as manifested by the conservation of spin angular momentum. Consequently, the DW moves in the direction of electron flow. The mechanism which involves the exchange of spin angular momentum between a spin polarized electron and DW magnetic moment, is known as adiabatic STT. When a DW is driven by an adiabatic STT, there exists an intrinsic pinning which governs the threshold current density [85]. 
The non-adiabatic STT, on the other hand, acts on a DW as if there is a torque acting due to an external magnetic field. Thus, the non-adiabatic STT moves a DW even if current density is below the threshold current density determined by adiabatic STT [97-98]. Like field induced DW motion, non-adiabatic STT induces DW motion through steady state motion at small current while it drives a DW through procession at a large current. It shows the presence of a threshold current density even for the DW motion induced by nonadiabatic STT. Though, the existence of non-adiabatic torque has been observed experimentally, the origin of non-adiabatic torque is not clearly understood. Much research has been devoted to understand this phenomenon.

\subsubsection{Spin-orbit torque driven domain wall motion}

Recently, it has been observed that the DWs in ultrathin ferromagnetic films sandwiched between a heavy metal and an oxide layer can move at very large speeds of the order of $\sim 400 \mathrm{~m} / \mathrm{s}$ and the direction of DW motion is opposite to the direction of electron flow [99-100]. For instance, Moore has shown the fast current induced DW motion at 100 $\mathrm{m} / \mathrm{s}$ in $\mathrm{Pt} / \mathrm{Co} / \mathrm{AlO}_{\mathrm{x}}$ nanostrips [101]. This observation cannot be explained in the paradigm of conventional STT, where the direction of DW motion is expected to be along the electron flow. Slonczewski-like and field-like torques, are instead introduced to explain this phenomenon and these are known to originate from spin-orbit torque at the interface of ferromagnet-heavy metal or ferromagnet-oxide. The LLG equation can be modified by incorporating these torques and is given by [102]

$$
\frac{\partial \vec{M}}{\partial t}=-\gamma \vec{M} \times \vec{H}_{e f f}+\alpha\left(\vec{M} \times \frac{\partial \vec{M}}{\partial t}\right)+\tau_{F} \sigma \times M+\tau_{T} M \times(\sigma \times M),
$$


where the effective field, $H_{\text {eff }}$, includes the contributions from spin-orbit interactions, $\sigma$ denotes the unit vectors in in-plane and perpendicular to the current and $\tau_{F}$ and $\tau_{T}$ are the field-like and Slonczewski-like torque coefficients, respectively. These torques are associated with field-like and Slonczewski-like effective fields respectively and are given by

$$
\begin{aligned}
& H_{F L} \sim \hat{z} \times j, \\
& H_{S L} \sim \vec{m} \times(\hat{z} \times j),
\end{aligned}
$$

where $z$ is the direction normal to the plane of the sample and $j$ is the current density.

\subsubsection{Feld-like torque}

The field-like torque acts as if there was an applied magnetic field acting on the system. The first experimental observation of the field-like torque was made by Miron et al. They have shown the existence of strong Rashba effective fields due to field-like torque $[98,54,103]$. They demonstrated that the domain nucleation can be modified by the presence of the effective field that acts in-plane and transverse to the current direction. From the quantitative analysis of the nucleations, they have estimated the amplitude of the $H_{F L}$ to be $\sim 1 \mathrm{~T}$. Since the Rashba effective field acts in-plane, it cannot induce DW motion. But the extraordinary DW motion is attributed to a combination of Rashba and non-adiabatic STT. They have suggested that the Rashba field locks the Bloch configuration such that the high speed DW motion can be attained through rigid translational motion. Fig. 1.19 shows the mechanism of current driven DW motion induced by the Rashba effect. 

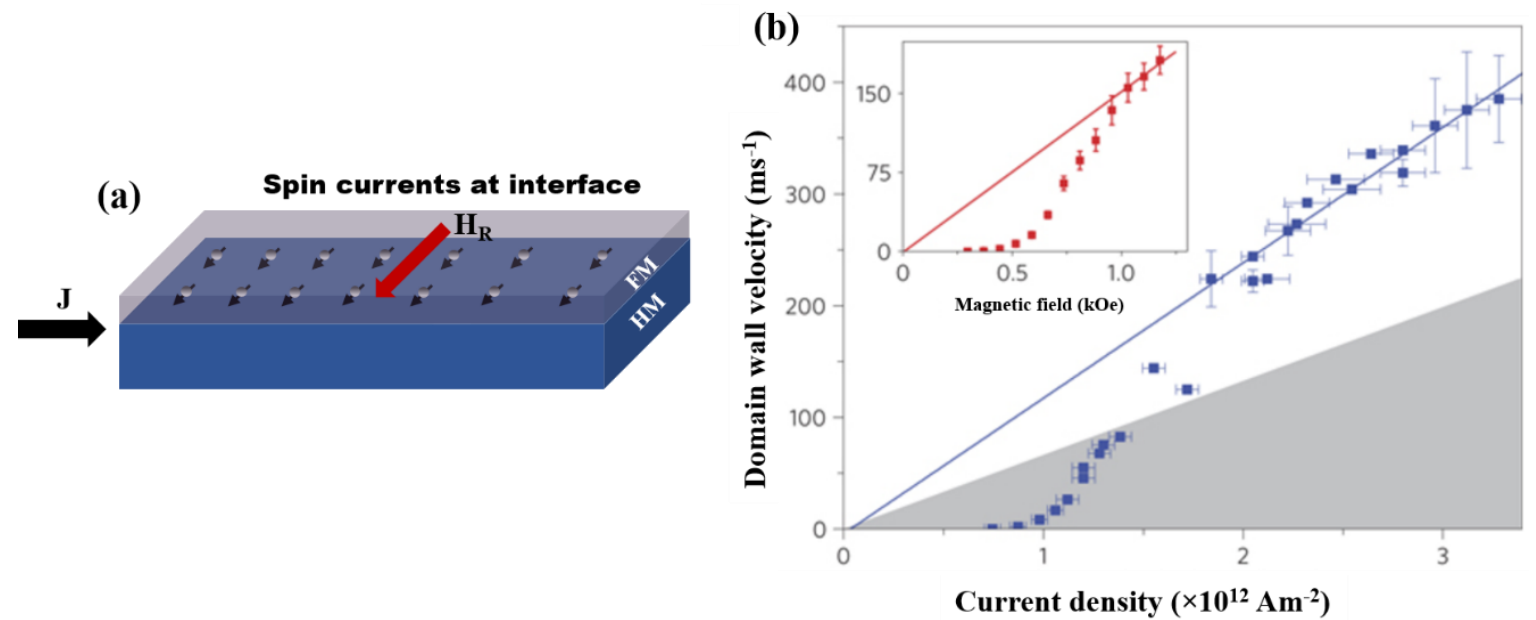

Fig. 1.19 (a) Schematic representation of Rashba effect, where the spin-orbit interaction at the interface of heavy metal (HM) and ferromagnetic material (FM) induces spin accumulation. The Rashba field $\left(H_{R}\right)$ acts as an in-plane transverse field. It locks the Bloch configuration, but does not drive the $D W$. (C) DW velocity as a function of current density. The solid line represents a linear fit to the DWflow regime while the shaded area represents the regions where the domain wall motion is turbulent motion. The inset shows a DW velocity as a function of magnetic field [103].

\subsubsection{Slonczewski-like torque}

Liu et al. have performed a series of experiments in similar heavy metal/ultrathin ferromagnet/oxide multilayer system to confirm the existence of Rashba torques [100, 104]. Instead, they proposed an alternative mechanism of spin Hall effect (SHE) to explain the observed results. Fig. 1.20 shows the schematic illustration of the spin Hall effect in a heavy metal. The SHE is the generation of spin currents which flows orthogonal to a charge current flowing through a heavy metal with strong spin-orbit coupling. 


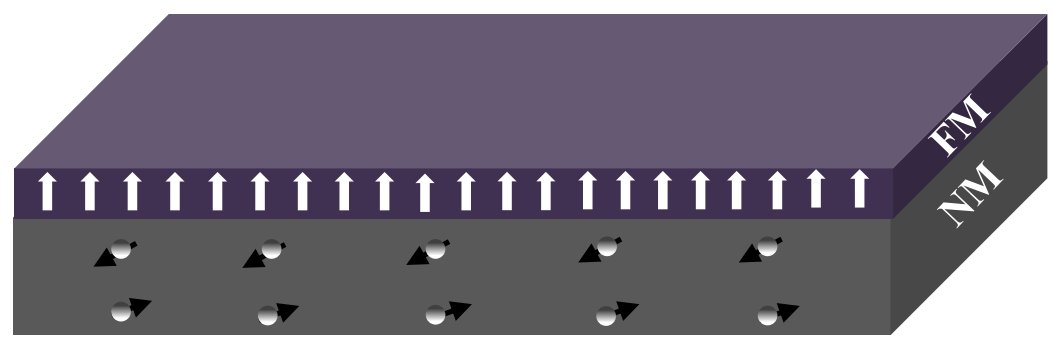

Fig. 1.20 Schematic illustration of the spin Hall effect in heavy metals. When an electric is passed through a heavy metal, spin currents are generated in the heavy metal and diffuses vertically into the adjacent ferromagnet.

The SHE is based on the spin dependent scattering of conduction electrons in the heavy metal. The heavy metals, Pt, Ta and $\mathrm{W}$, are known to possess strong spin-orbit coupling $[32,105]$. When an ultrathin ferromagnetic layer is contacted with a heavy metal, the spin currents diffuses vertically into the adjacent ferromagnet and exerts a torque known as Slonczewski-like torque. It is given by

$$
\begin{aligned}
& \tau_{S L}=-\gamma m \times\left(m \times H_{S H}\left(\hat{z} \times j_{e}\right)\right), \\
& H_{S H}=\frac{\hbar \theta_{S H}\left|j_{e}\right|}{2|e| M_{s} t},
\end{aligned}
$$

where $\theta_{S H}$ is the spin Hall angle, which can be defined as the ratio of the spin current $\left(I_{S}\right)$ to the charge current $\left(I_{e}\right)$. It measures the efficiency of the spin injection. The sign and amplitude of spin Hall angle depend on the thin film stack as well as the composition of the heavy metal layer. For instance, it has been shown that significant enhancement of Slonczewski-like torque can be achieved by placing different materials with opposite spin Hall angles [106]. 
However, the Slonczewski-like effective from SHE cannot drive a Bloch DW. For a Bloch wall with magnetization vector lying along $\pm y$, from eqn. 1.23 , the resulting torque, $\tau_{S L}$ would be zero. Néel configuration is thus required for the DW motion to be driven by SHE. However, a Néel configuration cannot be attained in out-of-plane magnetized materials because the formation of Néel wall would cost larger demagnetizing field.

However, a Néel wall can be stabilized by Dzyaloshinskii-Moriya interaction (DMI), which is an antisymmetric exchange interaction arises from the strong spin-orbit coupling [67, 107-108]. It induces an effective field ( $\left.\mathrm{H}_{\mathrm{DMI}}\right)$ that promotes Néel wall configuration. The presence of DMI can be inferred by applying an in-plane bias field together with an out-of-plane field that induces asymmetric domain expansion [109]. Under the in-plane bias field, $\uparrow \downarrow$ and $\downarrow \uparrow$ DWs experience different effective fields leading to different velocities [110]. It has been shown that DMI can stabilize Néel wall in $\mathrm{Pt} / \mathrm{CoFe} / \mathrm{MgO}$ and $\mathrm{Pt} / \mathrm{CoFe} / \mathrm{AlOx}$ thin film stacks $[33-34,111]$.

\subsection{Objectives of the thesis}

In this thesis, the dynamic properties of Néel DW in both in-plane and out-of-plane magnetic anisotropy materials have been systematically investigated with a view to implementing for DW logic and memory application. A novel technique which exploits the TDW internal profile for performing logic applications is proposed and experimentally verified. A method to deterministically generate single DW for on-chip applications is demonstrated. The dynamics of chiral Néel DWs driven by spin-orbit torque are investigated. 
The main objectives of this thesis are:

i. To propose and demonstrate a method to control the transverse DW profile inplane magnetized nanostructures

ii. To demonstrate a scheme to generate single domain wall in in-plane magnetized material deterministically and also to study the domain wall interaction.

iii. To explore dynamics of chiral Néel domain walls driven by spin-orbit torques in $\mathrm{Co} / \mathrm{Ni}$ heterostructures.

\subsection{Organization of the thesis}

- Chapter 1 provides an introduction and motivation for the work presented in the thesis and also introduces theoretical background for understanding the experimental observations presented in this thesis.

- $\quad$ Chapter 2 introduces various fabrication techniques and characterization tools that were employed during the course of this research work.

- Chapter 3 presents a novel method for the topological manipulation of transverse DW in in-plane magnetized nanostructures.

- $\quad$ Chapter 4 investigates transverse DWs interaction and develops a method to generate single DW deterministically by harnessing intrinsic stray field of the nanowire.

- Chapter 5 focuses on chiral Néel DW dynamics driven by spin-orbit torques in out-of-plane magnetized materials.

- Chapter 6 summarizes the main experimental results presented in this thesis and insights for possible future work are described. 


\section{References}

1. J. K. Ayling, R. D. Moore, G. K. Tu, Int. Solid-State Circuits Conf., Dig. Tech. Pap. 36 (1969).

2. R. Bez, E. Camerlenghi, A. Modelli, A. Visconti, Proc. IEEE 91, 489 (2003).

3. K. Kim, G. Koh, IEEE 7th Inter Conf. Solid-State Integer Circuits Technol. 662 (2004).

4. S. Aritome, IEEE IEDM Technical Digest, 763 (2000).

5. See www.itrs.net for International Roadmap for Semiconductors (ITRS) (2013).

6. M. Cornwell, Commun. ACM, 55, 59 (2012).

7. Zutic, J. Fabian, and S. Das Sarma, Rev. Mod. Phys., 76, 323 (2004).

8. J. Fabian, A. M. Abiaguea, C. Ertler, P. Stano, and T. Zutic, Acta Phys. Slovaca, 57, 565 (2007).

9. R. Jansen, Nat. Mater., II, 400 (2012).

10. A.V. Khvalkovskiy, D. Apalkov, S. Watts, R. Chepulskii, R. S. Beach, A. Ong, X. Tang, A. D. Smith, W. H. Butler, P. B. Visscher, D. Lottis, E. Chen, V. Nikitin and M. Krounbi, J. Phys. D: Appl. Phys. 46, 074001 (2013).

11. M. Hosomi, H. Yamagishi, T. Yamamoto, K. Bessho, Y. Higo, K. Yamane , H. Yamada , M. Shoji , H. Hachino, C. Fukumoto, H. Nagao and H. Kano, IEDM Tech. Dig., 459 (2005).

12. L. Kangho, S. H. Lee, IEEE Trans. Magn. 47, 131 (2011). 
13. D. C. Worledge, G. Hu, P. L. Trouilloud, D. W. Abraham, S. Brown, M. C. Gaidis, J. Nowak, E. J. O’Sullivan, R. P. Robertazzi, J. Z. Sun, and W. J. Gallagher, Proc. IEDM Tech. Dig. 296 (2010)

14. G. W. Burr, B. N. Kurdi, J. C. Scott, C. H. Lam, K. Gopalakrishnan, R. S. Shenoy, IBM J Res \& Dev 524, 449 (2008).

15. J. W. Lu, E. Chen, M. Kabir, M. R. Stan and S. A. Wolf, Int. Mater. Rev. 61, 456 (2016).

16. N. N. Mojumder, K. Roy and D. W. Abraham, IEEE Trans. Magn. 49483 (2013).

17. M. Cubukcu, O. Boulle, N. Mikuszeit, C. Hamelin, T. Bracher, N. Lamard, M. C. Cyrille, L. B. Prejbeanu, K. Garello, K. Garello, I. M. Miron, O. Klein, G. D. Loubens, V. V. Naletov, J. Langer, B. Ocker, P. Gambardella and G. Gaudin, arXiv preprint arXiv:1509.02375 (2015).

18. M. Cubukcu, O. Boulle, M. Drouard, K. Garello, C. O. Avci, I. M. Miron, J. Langer, B. Ocker, P. Gambardella and G. Gaudin, Appl. Phys. Lett. 104, 042406 (2014).

19. S. S. P. Parkin, M. Hayashi, and L. Thomas, Science 320, 190 (2008).

20. N. Vernier, D. A. Allwood, D. Atkinson, M. D. Coke, and R. P. Cowburn, Europhys. Lett. 65, 526 (2004).

21. A. Yamaguchi, T. Ono, S. Nasu, K. Miyake, K. Mibu, and T. Shinjo, Phys. Rev. Lett. 92, 077205 (2004).

22. E. Saitoh, H. Miyajima, T. Yamaoka, and G. Tatara, Nature 432, 203 (2004). 
23. M. Klaui, M. Laufenberg, L. Heyne, D. Backes, U. Rudiger, C. A. F. Vaz, J. A. C. Bland, L. J. Heyderman, S. Cherifi, A., Locatelli, T. O. Mentes, and L. Aballe, Appl. Phys. Lett. 88, 232507 (2006).

24. M. Klaui, P. O. Jubert, R. Allenspach, A. Bischof, J. A. C. Bland, G. Faini, U. Rudiger, C. A. F. Vaz, L. Vila, and C. Vouille, Phys. Rev. Lett. 95, 026601 (2005).

25. L. Heyne, M. Kläui, D. Backes, T. A. Moore, S. Krzyk, U. Rüdiger, L. J. Heyderman, A. Fraile Rodríguez, F. Nolting, T. O. Mentes, M. Á. Niño, A. Locatelli, K. Kirsch, and R. Mattheis, Phys. Rev. Lett. 100, 0666032008.

26. S. W. Jung, W. Kim, T.-D. Lee, K.-J. Lee, and H.-W. Lee, Appl. Phys. Lett. 92, 2025082008.

27. S. Fukami, T. Suzuki, N. Ohshima, K. Nagahara, and N. Ishiwata, J. Appl. Phys. 103, 07E718 2008.

28. Y. Yoshimura, T. Koyama, D. Chiba, Y. Nakatani, S. Fukami, M. Yamanouchi, H. Ohno, and T. Ono, Appl. Phys. Express 5, 063001 (2012).

29. K. Ueda, T. Koyama, R. Hiramatsu, D. Chiba, S. Fumani, H. Tanigawa, T. Suzuki, N. Ohshima, N. Ishiwata, Y. Nakatani, K. Kobayashi, and T. Ono, Appl. Phys. Lett. 100, 202407 (2012)

30. I. M. Miron, P.-J. Zermatten, G. Gaudin, S. Auffret, B. Rodmacq, and A. Schuhl, Phys. Rev. Lett. 102, 1372022009. 
31. L. S. E. Alvarez, K.-Y. Wang, S. Lepadatu, S. Landi, S. J. Bending, and C. H. Marrows, Phys. Rev. Lett. 104, 1372052010.

32. P. P. J. Hazeen, E. Mure, J. H. Franken, R. Lavrijsen, H. J. M. Swagten, and B. Koopmans, Nat. Mater. 12, 299 (2013).

33. S. Emori, U. Bauer, S. M. Ahn, E. Martinez, and G. S. D. Beach, Nat. Mater. 12, 611 (2013).

34. K. S. Ryu, L. Thomas, S.-H. Yang, and S. S. P. Parkin, Nat. Nanotechnol. 8, 527 (2013)

35. S. Glathe, M. Zeisberger, U. Hubner, R. Mattheis and D. V. Berkov, Phys. Rev. $B$ 81, 020412 (2010).

36. O. A. Tretiakov, D. Clarke, G. W. Chern,Y. B. Bazaliy and O.Tchernyshyov, Phys. Rev. Lett. 100, 127204 (2008).

37. T. Ono, H. Miyajima, K. Shigeto, K. Mibu, N. Hosoito and T. Shinjo, Science 284, 468 (1999).

38. D.Atkinson, D. A. Allowed, G. Xiong, M. D. Cooke, C. C. Faulkner and R. P. Cowburn. Nat. Mater. 2, 85 (2003).

39. L. K. Bogart, D. Atkinson, K. OShea, D. McGrouther and S. McVitie, Phys. Rev. B 79, 054414 (2009).

40. J. C. Slonczewski, J. Magn. Magn. Mater. 159, L1 (1996) 
41. A.J. Annunziata, M.C. Gaidis, L. Thomas, C.W. Chien, C.C. Hung, P. Chevalier, E.J. O’Sullivan, J.P. Hummel, E.A. Josef, Y. Zhu, T. Topuria, E. Delenia, P.M. Rice, S.S.P. Parkin, and W.J. Gallagher, Proc. IEDM 539, (2011).

42. J. H. Franken, H. J. M. Swagten and B. Koopmans, Nature Nanotech. 7, 499 (2012).

43. D. A. Allwood, G. Xiong, C. C. Faulkner, D. Atkinson, D. Petit and R. P. Cowburn, Science 309, 1688 (2005).

44. D. A. Allwood, G. Xiong, M. D. Cooke, C. C. Faulkner, D. Atkinson, N.vVernier, R. P. Cowburn Science 296, 2003 (2002).

45. M. Hayashi, L. Thomas, R. Moriya, C. Rettner and S. S. Parkin, Science 320, 209 (2008).

46. K. J. Kim, J. C. Lee, S. J. Yun, G. H. Gim, K. S. Lee, S. B. Choe and K H. Shin, Appl. Phys. Exp. 3, 8 (2010).

47. X. Jiang, L. Thomas, R. Moriya, M. Hayashi, B. Bergman, C. Rettner and S. S. Parkin, Nature Commun. 1, 25 (2010).

48. C. Burrowes, A. P. Mihai, D. Ravelosona, J. V. Kim, C. Chappert, L. Vila, A. Marty, Y. Samson, F. Garcia-Sanchez, L. D. Buda-Prejbeanu, I. Tudosa, E. E. Fullerton \& J.-P. Attané, Nature Phy. 6, 17 (2010).

49. M. Klaui, P. O. Jubert, R. Allenspach, A. Bischof, J. A. C. Bland, G. Faini, U. Rudiger, C. A. F. Vaz, L. Vila, and C. Vouille, Phys. Rev. Lett. 95, 026601 (2005).

50. L. Thomas, S. H. Yang, K. S. Ryu, B. Hughes, C. Rettner, D. S. WangC. H. Tsai, K. H. Shen and S. S.S. Parkin, IEEE IEDM 24, 21 (2011). 
51. A. J. Annunziata, M. C. Gaidis, L. Thomas, C. W. Chien, C. C. Hung, P. Chevalier, E. J. O'Sullivan, J. P. Hummel, E. A. Joseph, Y. Zhu, T. Topuria, E. Delenia, P. M. Rice, S. S. P. Parkin, W. J. Gallagher, IEEE IEDM 24, 31 (2011).

52. Y Zhang, W. S. Zhao, D. Ravelosona, J. O. Klein, J. V. Kim and C. Chappert, J. Appl. Phys. 111, 093925 (2012).

53. D. Chiba, G. Yamada, T. Koyama, K. Ueda, H. Tanigawa, S. Fukami, T. Suzuki, N. Oshima, N. Ishiwata, Y. Nakatani and T. Ono, Appl. Phys. Exp. 3, 073004 (2010).

54. I. M. Miron, T. Moore, H. Szambolics, S. Auffret, B. Rodmacq, S. Pizzni, J. Vogel, M. Bonfim, A. Schuhl and G. Gaudin, Nat. Mater. 10, 419-423 (2011).

55. K. S. Ryu, L. Thomas, S. H. Yang and S. S. Parkin, Nat. Nanotechnol. 8, 527 (2013) S. H. Yang, K. S. Ryu and S. S. Parkin, Nat. Nanotechnol. 10, 221 (2015).

56. S. Fukami, T. Suzuki, K. Nagahara, N. Ohshima, Y. Ozaki, S. Saito, R. Nebashi, N. Sakimura, H. Honjo, K. Mori, C. Igarashi, S. Miura, N. Ishiwata, and T. Sugibayashi, Symposium on VLSI technology 230 (2009).

57. U. Bauer, S. Emori and G. S. D. Beach, Nat. Nanotechnol. 8, 411 (2013).

58. K. W. Moon, D. H. Kim, S. C. Yoo, S. G. Je, By. S. Chun, W. Kim, B. C. Min, C. Hwang and S. B. Choe, Scientific Reports 5, 9166 (2015).

59. S. Goolaup, M. Ramu, C. Murapaka and W. S. Lew, Scientific Reports 5, 9603 (2015). 
60. M. Chandra Sekhar, P. Sethi, S. Goolaup, and W. S. Lew, Scientific Reports, 6, 21099 (2016).

61. P. Weiss, Bulletin de la Societe Francaise de Physique 1, 95 (1907).

62. W. Heisenber, Zeitschrift fur Physik 49, 619 (1928).

63. W. F. Brown, Jr.: Micromagnetics (Wiley, New York, 1963).

64. E. Martinez, J. Phys-Condens. Mat. 24, 024206 (2012).

65. Blundell, S. Magnetism in Condensed Matter. (Oxford, 2003).

66. I. Dzyaloshinskii, Phys. Chem. Solids 4, 241 (1958).

67. T. Moriya, Phys. Rev. 120, 91 (1960).

68. B. N. Filippov, Physics of Solid state 542407 (2012).

69. M. Klaui, J. of Phys.: Condensed Matter 20, 313001(2008).

70. G. Catalan, J. Seidel, R. Ramesh, and J. F. Scott, Rev. Mod. Phys. 84, 119 (2012).

71. M. Redjdal, J. Giusti, M. F. Ruane, and F. B. Humphrey, J. Appl. Phys. 91, $7547(2002)$.

72. R.C. O’Handley. Modern magnetic materials principles and applications. Wiley (2000).

73. R. D. McMichael, and M. J. Donahue, IEEE Trans. Magn. 33, 4167 (1997).

74. G.S.D. Beach, M. Tsoi, J. L. Erskine, J. Magn. Magn. Mater. 3201272 (2008)

75. H. T. Zeng, D. Petit, L. O'Brien, D. Read, E. R. Lewis, and R. P. Cowburn, J. Magn. Magn. Mater. 322, 2010 (2010).

76. L. OBrien, D. Petit, H. T. Zeng, E. R. Lewis, J. Sampaio, A. V. Jausovec, D. E. Read, and R. P. Cowburn, Phys. Rev. Lett. 103, 077206 (2009). 
77. L. OBrien, A. Beguivin, A. Fernandez-Pacheco, D. Petit, D. E. Read, and R. P. Cowburn, Appl. Phys. Lett. 101, 062415 (2012).

78. I. Purnama, M. Chandra Sekhar, S. Goolaup, and W. S. Lew, Appl. Phys. Lett. 99, 152501 (2011).

79. O. Tchernyshyov and G. W. Chern, Phys. Rev. Lett. 95, 197204 (2005).

80. Y. Shen, O. Petrova, P. Mellado, S. Daunheimer, J. Cumings, and O.Tchernyshyov, New J. of Phys.14, 035022 (2012).

81. P. M. Chaikin and T. C. Lubensky, Principles of Condensed Matter Physics (Cambridge Univ. Press, 2000).

82. A. Kunz. Appl.Phys. Lett. 94, 132502 (2009).

83. L. Thomas, M. Hayashi,B. Moriya, C. Rettner and S. S. P. Parkin, Nat. Comm. 3, 810 (2012).

84. L. OBrien, E. R. Lewis, A. F. Pacheco, D. Petit, R. P. Cowburn, J. Sampaio, and D. E. Read, Phys. Rev. Lett. 108, 187202 (2012).

85. T. Koyama, D. Chiba, K. Ueda, K. Kondou, H. Tanigawa, S. Fukami, T. Suzuki, N. Ohshima, N. Ishiwata, Y. Nakatani, K. Kobayashi and T. One, Nature Materials 10, 194 (2011).

86. M. Heide, G. Bihlmayer and S. Blugel, Phys. Rev. B 78, 140403 (2008).

87. G. Chen, J. Zhu, A. Quesada, J. Li, A. T. N. Diaye, Y. Huo, T. P. Ma, Y. Chen, H. N. Kwon, C. Won, Z. Q. Qiu, A. K. Schmid and Y. Z. Wu, Phys. Rev. Lett. 110, 177204 (2013).

88. G. Chen, T. P. Mam, A. T. N. Diaye, H. N. Kwon, C. Won and Y. Wu, Nat. Commun. 4, 2671 (2013). 
89. L. Landau and E. Lifshitz Physikalische Zeitschrift der Sowjetunion, 8, 153 (1935).

90. T. L. Gilbert, IEEE Trans. Mag., 40, 3443 (2004).

91. N. L. Schryer, L. R. Walker, J. of Appl. Phys. 45, 5406-5421 (1974).

92. L. Berger, J. of Appl. Phys. 49, 2156 (1978).

93. P. P. Freitas and L. Berger, J. Appl. Phys. 571266 (1985).

94. C. Y. Hung and L. Berger, J. Appl. Phys. 63, 4276 (1988).

95. J. Grollier, P. Boulenc, V. Cros, A. Hamzić, , A. Vaurès and A. Fert G. Faini, Appl. Phys. Lett. 83, 509 (2003).

96. M. Tsoi, R. E. Fontana and S. S. Parkin, Appl. Phys. Lett. 83, 2617 (2003).

97. S. Zhang and Z. Li, Phys. Rev. Lett. 93, 127204 (2004).

98. A. Thiaville, Y. Nakatani, J. Miltat and Y. Suzuki, Euro Phys. Lett. 69990 (2005).

99. I. M. Miron, K. Garello, G. Gaudin, P. J. Zermatten, M. V. Costache, S. Bandiera, B. Rodmacq ,A. Schuhl and P. Gambardella, Nature 476, 189 (2011).

100. L. Liu, O. J. Lee, T. J. Gudmundsen, D. C. Ralph and R. Buhrman, Phys. Rev. Lett. 109, 1-5 (2012).

101. T. A. Moore, I. M. Miron, G. Gaudin, G. Serret, S. Auffret, B. Rodmacq, A. Schuhl, S. Pizzini, J. Vogel, and M. Bonfim Appl. Phys. Lett. 93, 262504 (2008).

102. X. Fan, H. Celik, J. Wu, C. Ni, K. J. Lee, V.O. Lorenz and J.Q. Xiao, Nat. Commun. 5, 3042 (2014). 
103. I. M. Miron, G. Gaudin, S. Auffret, B. Rodmacq, A. Schuhl, S. Pizzini, J. Vogel, P. Gambardella, Nat. Mater. 9, 230-234 (2010).

104. L. Liu, C.F. Pai, Y. Li, H.W. Tseng, D.C Ralph and R.A. Buhrman. Science 336, 555-558 (2012).

105. A. V. Khvalkovskiy, V. Cros, D. Apalkov, V. Nikitin, M. Krounbi, K. A. Zvezdin, A. Anane, J. Grollier, and A. Fert, Phys. Rev. B 87, 020402 (2013).

106. S. Woo, M. Mann, A. J. Tan, L. Caretta and G. S. D. Beach, Appl. Phys. Lett. 105, 21404 (2014).

107. M. Heide, G. Bihlmayer and S. Blügel, Phys. Rev. B 78, 140403(2008).

108. A. Thiaville, S. Rohart, E. Jue, V. Cros and A. Fert, Europhys. Lett. 100, $57002(2012)$

109. S. G. Je, D.H. Kim, S.C. Yoo, B.C. Min, K. J. Lee, and S.C. Choe. Phys. Rev. B 88, 214401 (2013).

110. A. Hrabec, N. A. Porter, A. Wells, M. J. Benitez, G. Burnell, S. McVitie, D. McGrouther and T. A. Moore, and C. H. Marrows. Phys. Rev. B 90, 020402 (R) (2014).

111. S. Emori, E. Martinez, K.J. Lee, H.W. Lee, U. Bauer, S.M. Ahn, P. Agrawal, D. C. Bono, and G. S. D. Beach, Phys. Rev. B 90184427 (2014). 


\section{Chapter 2}

\section{Experimental techniques}

\subsection{Introduction}

This chapter provides the details of various fabrication techniques and characterization tools employed in this thesis. This chapter is mainly divided into two sections. The first section describes the sample fabrication which involves the deposition of thin films and patterning them into nanostructures followed by the deposition of electrical contacts. Magnetron sputtering was used for depositing both thin films and electrical contacts. Electron beam lithography was employed to pattern the nanostructures. In the second section, various characterization tools to investigate the structural properties and the magnetic properties are discussed. Scanning electron microscopy was used to study the structural properties of the patterned nanostructures. Magnetic force microscopy was employed to image the magnetization configurations of the devices. Further, probe station was extensively used to study the domain wall generation and dynamics. Finally, method to measure strength and sign of DMI in ultrathin layers of perpendicularly magnetized materials was described.

\subsection{Sample fabrication}

Before providing the detailed description of each fabrication technique, an outline of the sample cleaning was presented at first as it affects the device performance. The thin film stacks were deposited on amorphous $\mathrm{SiO}_{2}(300 \mathrm{~nm}) / \mathrm{Si}$ substrates which were grown by thermal oxidation. The amorphous nature of $\mathrm{SiO}_{2}$ prevents the effects of underlying crystalline $\mathrm{Si}$ affecting the growth of subsequent layers. It also acts as an electrically insulting layer for electrical measurements. 
Firstly, the substrates were ultra-sonicated in acetone for 20 minutes to ensure that contaminants were removed. Secondly, the substrates were ultra-sonicated in IPA for 20 minutes. This step removes any residual of acetone from the sample. Finally, $\mathrm{N}_{2}$ blow off was employed to remove any particulate material from the substrate.

\subsection{Thin film deposition by magnetron sputtering}

Among various vacuum deposition techniques available today for depositing thin films, sputtering technique is the most capable technique to deposit thin films of almost any material [1-2]. Fig. 2.1 shows schematic illustration of sputtering process. The sputtering is a physical vapor deposition (PVD) technique wherein atoms are ejected from the target material upon high energy particle bombardment, which then condense onto a substrate as a thin film [3-4].

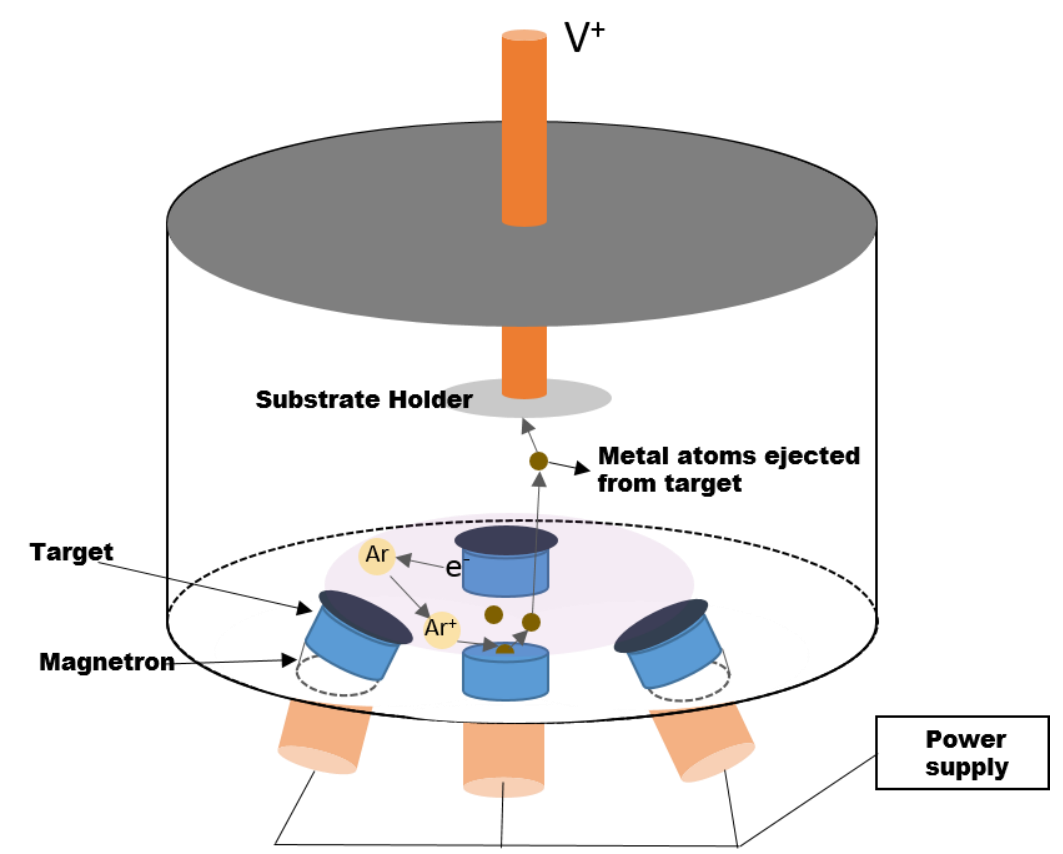

Fig. 2.1 Schematic of a magnetron sputtering deposition process. Permanent magnets are placed in close proximity to the target. The substrate holder is maintained at positive potential with respect to the target. 
The sputtering deposition technique, in particular magnetron sputtering has been widely used to deposit thin films of ferromagnetic materials for better uniformity, density, and purity [5-8]. Ultra-high purity Ar gas (99.999\%) is used at high vacuum for the sputtering process. When a strong voltage is applied to the target, the Ar gas is ionised and forms a $\mathrm{Ar}^{+}$plasma in the chamber. Since, the target is maintained at negative potential relative to the substrate, the Ar ions are continuously accelerated towards the sputtering target. When these Ar ions bombard on the target surface, the target atoms are ejected from the surface. These atoms traverse in the chamber and subsequently deposited onto the substrate as a thin film.

As shown in Fig. 2.2, magnetic poles are placed in close proximity to the target material. A closed loop of magnetic flux is then generated and extending from one pole of a magnet to the other pole. The spiral motion of the electron along this path increases the path length of the electron thereby increasing the sputtering rate [5].

\section{Magnetic flux}

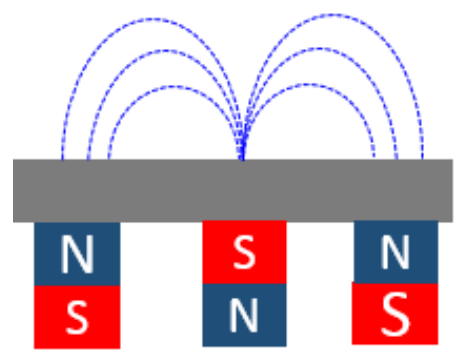

Magnetic poles

Fig. 2.2 Magnetic flux generated on the target surface forms a closed-loop to confine electrons as well as to increase their path lengths in order to maximize the probability of ionizing collisions. 
The thickness uniformity of the film is determined by the working pressure [5]. By controlling the Ar gas flow, the depositions can be carried out at low working pressures which ensures better quality of the film. However, a minimum working pressure is required to obtain a stable plasma.

As shown in Fig. 2.3, all thin films investigated in this thesis were deposited by ultrahigh vacuum magnetron sputtering system, with a base pressure of $\sim 2 \times 10^{-8}$ Torr [9]. The working pressure of 2-3 mTorr was maintained by a controlling Ar gas flow. The sputtering system is equipped with a load lock chamber to transfer the samples without breaking the vacuum. The system is capable of DC and RF sputtering. However, all the films studied in this thesis were deposited by using DC power supply. The chamber has a rotating sample stage which improves the uniformity in the film thickness.

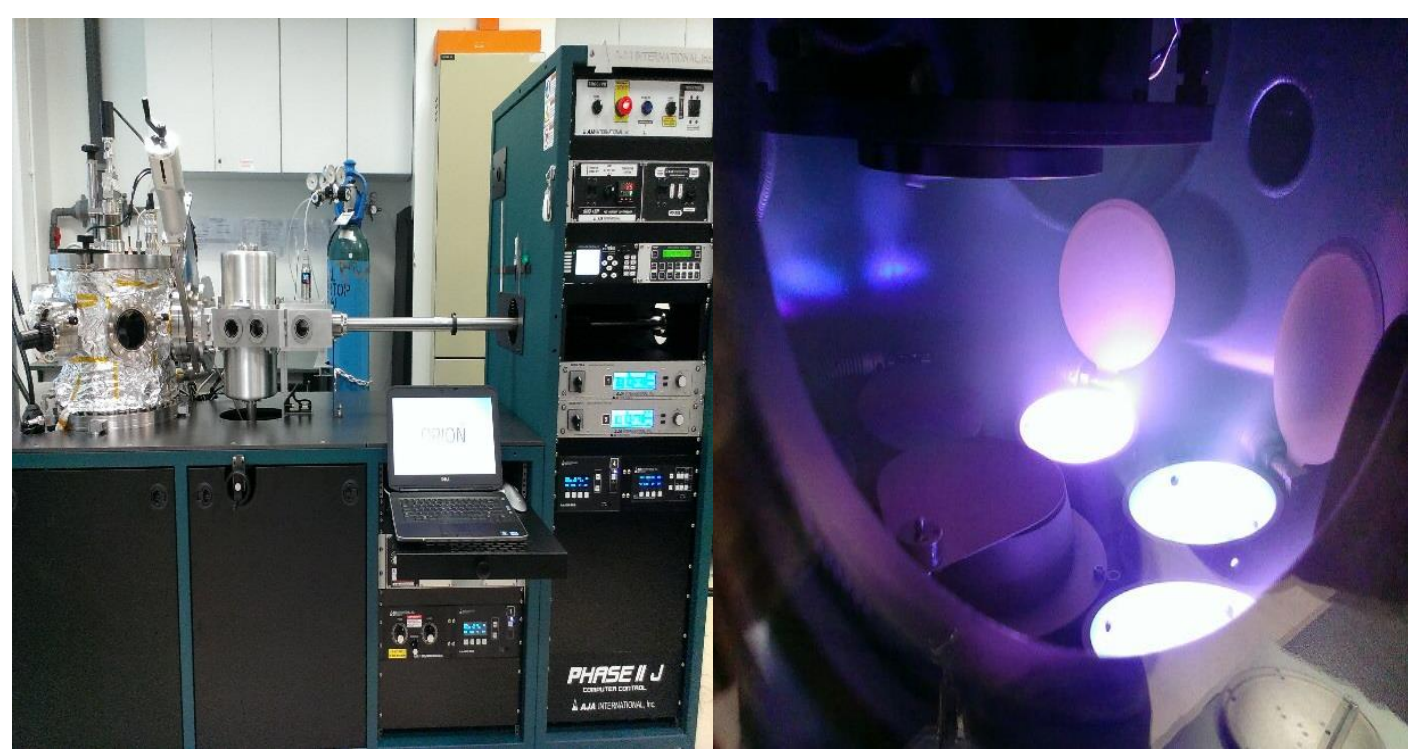

Fig. 2.3 A sputtering deposition system used for growing the films presented in this thesis. The power supply cabinet, which includes both DC and RF power supplies and deposition chamber [9]. Ar plasma generation can be seen on the right side. 
The deposition rates are typically scales with the power. To determine the deposition rates of different materials, thin films with a fixed sputtering time were deposited. Atomic force microscopy (AFM) was then used to measure the thickness of individual film. The deposition rates can be obtained subsequently by taking the ratio of film thickness to sputtering time.

\subsection{Device fabrication}

After the deposition of thin film stacks by magnetron sputtering, the samples were patterned into nanostructures using electron beam lithography technique (EBL) [10-13]. EBL is one of the key fabrication technique to pattern nanostructures down to sub 10-nm, owing to shorter wavelength of electrons that it employs [14-17]. A focussed beam of electrons is guided over a surface, which is covered with EBL resist. The solubility of the resist can be controlled by tuning the energy (dose) of the exposed beam [18]. When the electron beam is exposed on the negative tone EBL resist, the exposed region gest hardened and remains after the development. On the other hand, for positive tone EBL resist, the polymer which is exposed by the electron beam will be washed away after the development. The EBL cannot be used for high-volume manufacturing because of the low throughput. Fig. 2.4 shows the EBL system used for patterning the nanostructures presented in this thesis. In this thesis, a negative tone EBL resist, MAN 2403, was used to pattern the nanostructures of sub-100 nm while a positive tone EBL resist, 950 PMMA, was used for patterning the electrical contacts. 


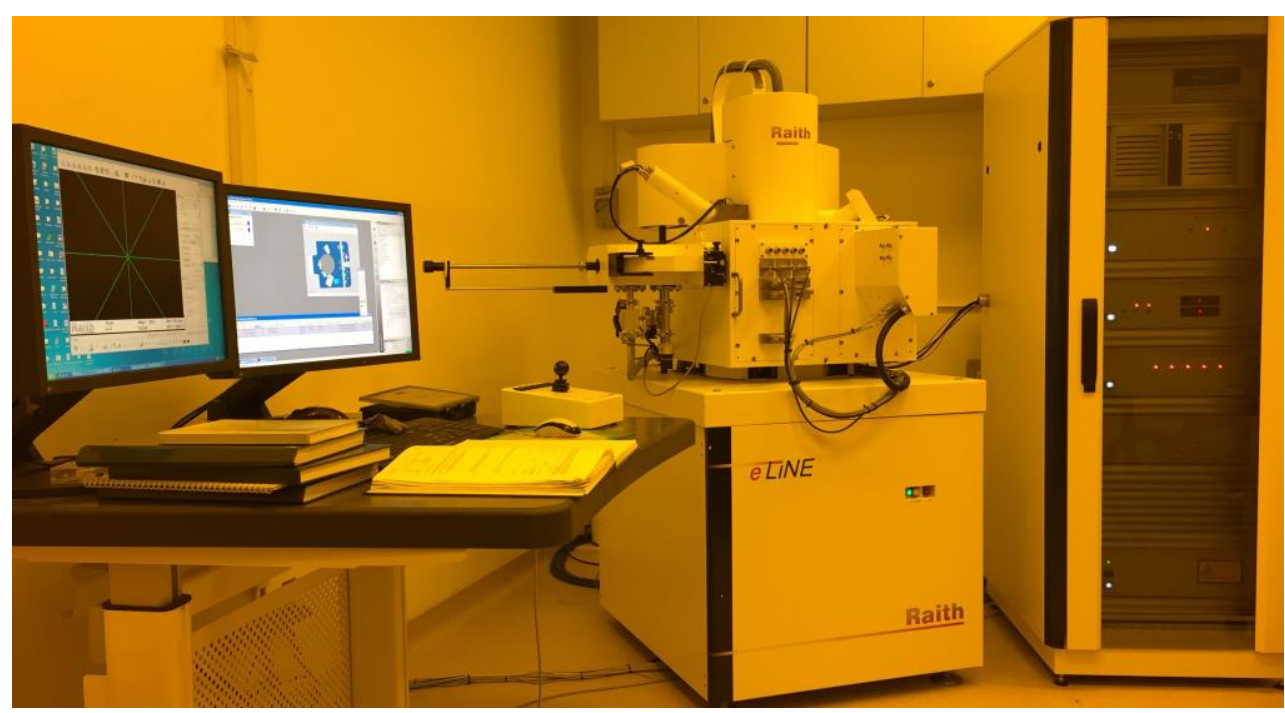

Fig. 2.4 Raith e-line 150 lithography system is used for patterning the nanostructures [19].

Fig. 2.5 shows step-by-step process of fabricating the nanostructures using MAN2403 (negative e-beam resist).

1. Prior to the resist coating, the sample was cleaned in acetone and IPA each for 10 mins using ultrasonication followed by pre-baking at $100^{\circ} \mathrm{C}$ for 5 mins.

2. MAN 2403 was spin-coated at $6000 \mathrm{rpm}$ for $60 \mathrm{sec}$. The expected resist thickness is $\sim 200 \mathrm{~nm}$, which is sufficient to acts as an etching mask. The substrate was post baked at $100^{\circ} \mathrm{C}$ for $60 \mathrm{sec}$. The sample was then loaded into the EBL chamber via load lock chamber.

3. Raith EBL 150 system was used to pattern the samples. The exposure parameters are: $20 \mathrm{keV}$ acceleration voltage, $7 \mu \mathrm{m}$ aperture, $10 \mathrm{~nm}$ working distance and 100 $\mu \mathrm{m} \times 100 \mu \mathrm{m}$ writing field. The exposure was performed in the area scan mode to obtain better resolution. Prior to the exposure, the desired patterns were drawn using the e-line software and then are written onto the resist by electron beam exposure.

4. Soak the exposed sample in MAD 525 developer for $90 \mathrm{sec}$ and rinse it with DI water for 5 mins. The sample was then blow dried with $\mathrm{N}_{2}$ gas. 


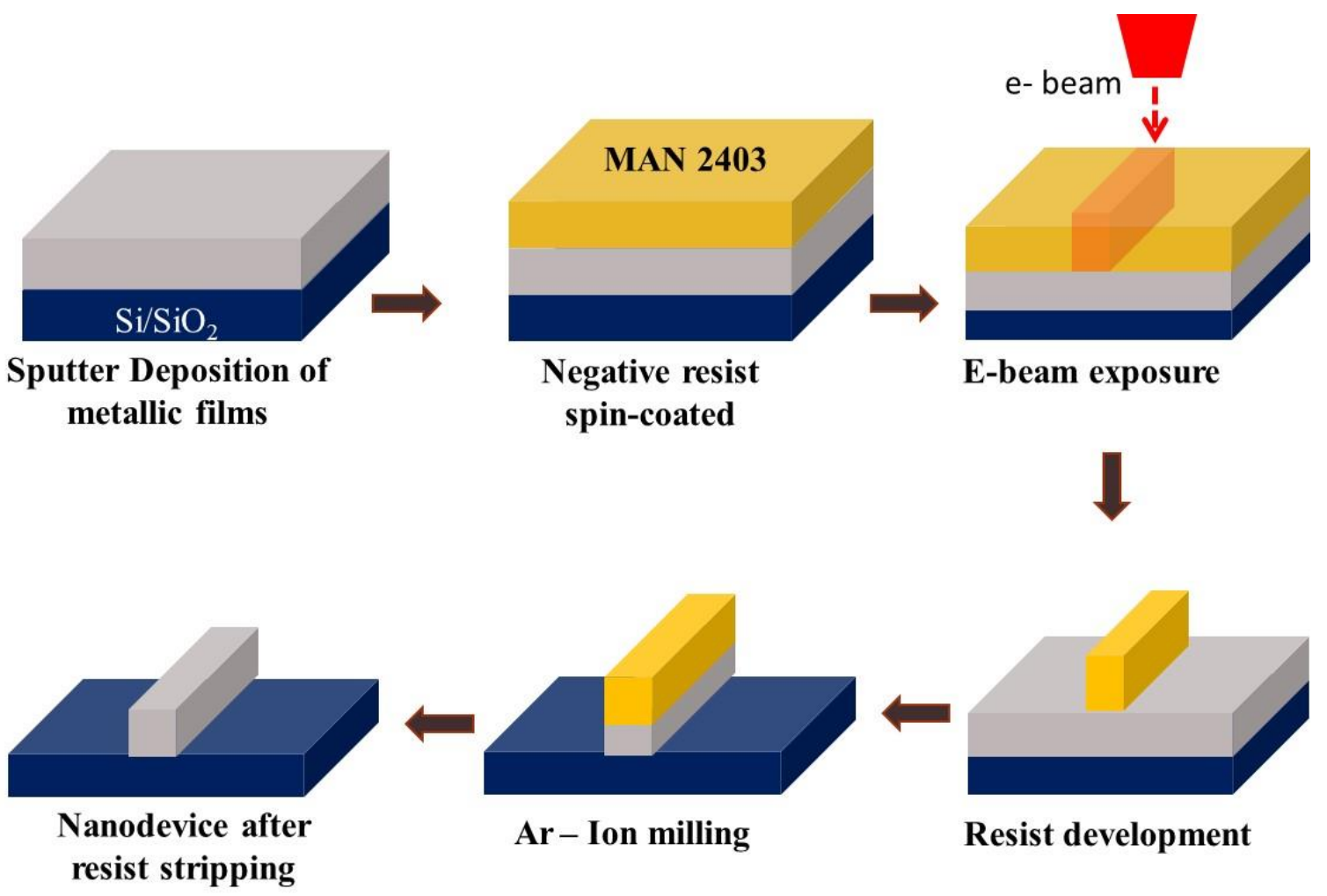

Fig. 2.5 Step-by-step process for e-beam exposure for negative resist.

5. The unprotected parts of the sample were removed by Ar ion milling, which produces nanostructures with sharp side walls.

6. The residual particles of the resist that left on the sample after the ion milling were removed by ultrasonic-stripping in MR remover for $4 \mathrm{hrs}$. The sample was then blow dried with $\mathrm{N}_{2}$ gas.

To fabricate electrical contacts onto the nanostructures, a positive tone EBL resist (950 PMMA) was used. The method for fabricating the contacts is different from that of the nanostructures. Fig. 2.6 shows the schematic of the fabrication using positive resist.

1. The sample was ultrasonicated in acetone and IPA for 10 mins each.

2. A layer of PMMA resist was spun onto the sample at $4000 \mathrm{rpm}$ for $45 \mathrm{sec}$, which ensures a resist thickness of $140 \mathrm{~nm}$. The sample was then baked at $180^{\circ} \mathrm{c}$ for $5 \mathrm{~min}$. 
3. The sample was loaded into the EBL chamber and subsequently exposed by e-beam.

4. The exposed part was developed by dipping the sample into a solution of MIBK:IPA (1:3) for $30 \mathrm{sec}$ followed by a cleaning in IPA for $30 \mathrm{sec}$ and blow dried by $\mathrm{N}_{2}$ gun.

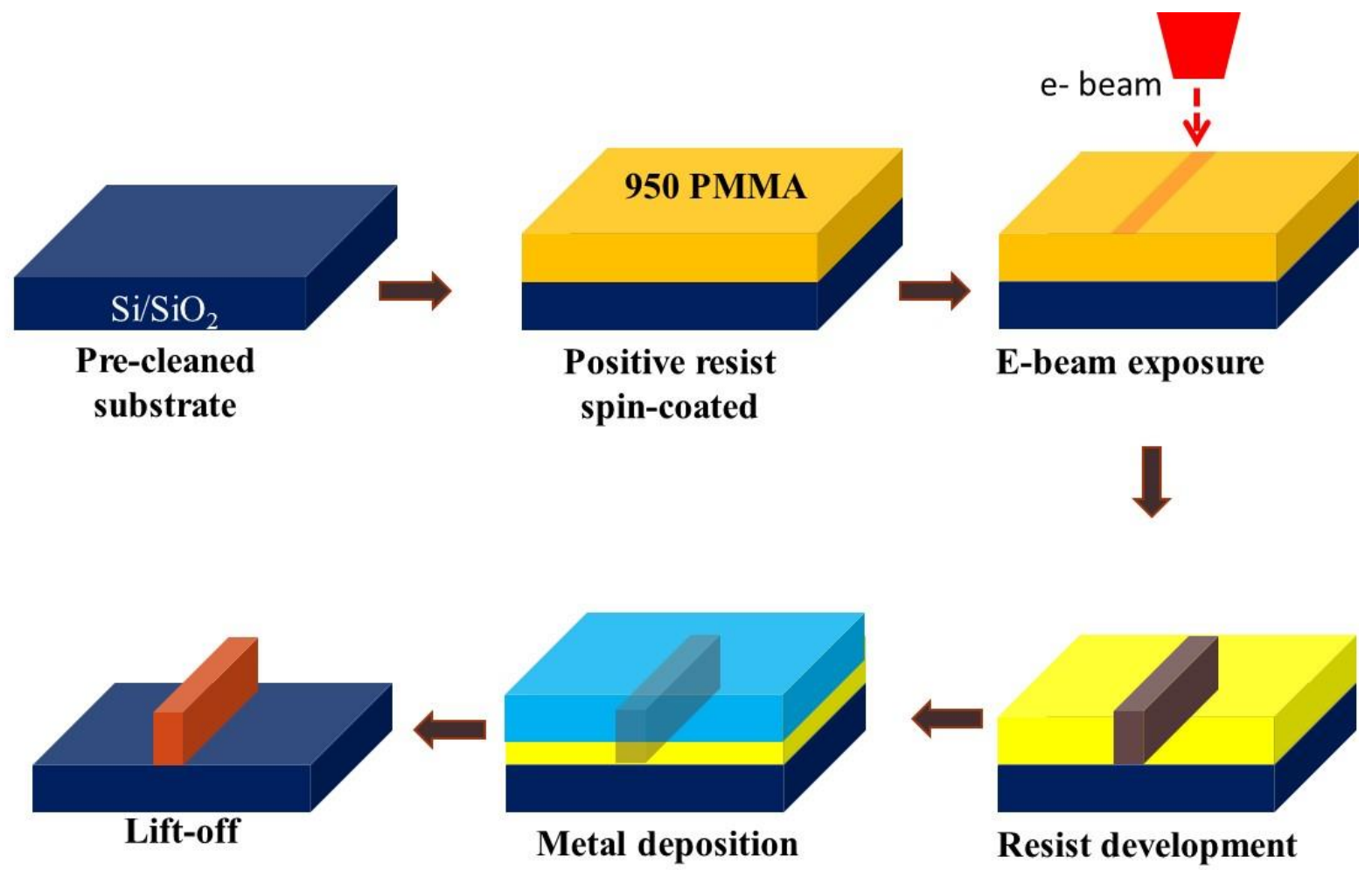

Fig. 2.6 step-by-step process of e-beam patterning using positive resist. The positive resist was used for patterning electrical contacts for the devices.

5. The sample was then transferred inside the magnetron sputtering for depositing metal contacts. Prior to the deposition, portion of the nanowire was etched using reverse sputtering to provide better contact resistance.

6. A metal lift-off was done by ultrasonicating the sample in warm acetone. 


\subsection{Characterization tools}

This section describes the various characterization tools used to probe the properties of nanostructures, namely: scanning electron microscopy, magnetic force microscopy, probe station measurements.

\subsubsection{Scanning electron microscopy}

Scanning electron microscope (SEM) is a powerful characterization for imaging the structures ranging from $\mathrm{mm}$ to $\mathrm{nm}$ scale [20-21]. The image resolution featured by an SEM depends not only on the property of the electron probe but also on the electron sample interaction. SEM uses basically the same principle used in EBL systems. Focussed beam of high-energy electrons in SEM carry significant amounts of kinetic energy. Up on interaction with the sample, this energy is dissipated into variety of signals at the surface of sample, which in turn provides the information about the sample. Fig. 2.7 shows the schematics of the sample-electron interaction. For instance, secondary electrons provide the topographical information of the sample, Auger electron and X-rays carry characteristic energy and provides information about the elemental composition of the sample [22].

Fig. 2.8 shows the schematic of a typical SEM chamber. An electron gun on the top produces beam of electrons via thermionic emission. The anode plate forces the electrons to follow a vertical path through a microscope. After traversing through electromagnetic fields and lenses, the beam becomes high energy focussed beam. Once the beam hits the sample, different waves and particles are emitted. The detector collect these signals and subsequently produce the final image of the sample. The backscattered electron detector is a solid-state type device, which detects elastically scattered electron and gives useful information about the composition and surface topography of the sample. 


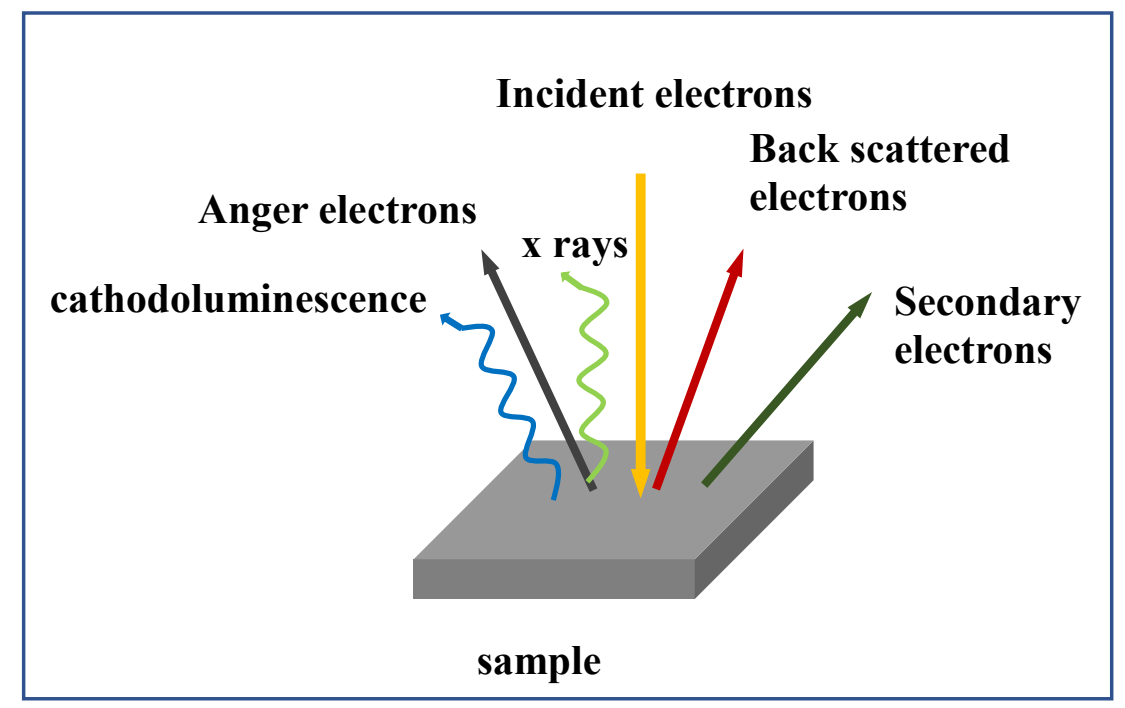

Fig. 2.7 Schematic of the electron-matter interaction. Upon interaction, energy dissipation leads to different signal from sample. Secondary electron, gives information about the topology while backscattered electrons give chemical composition.

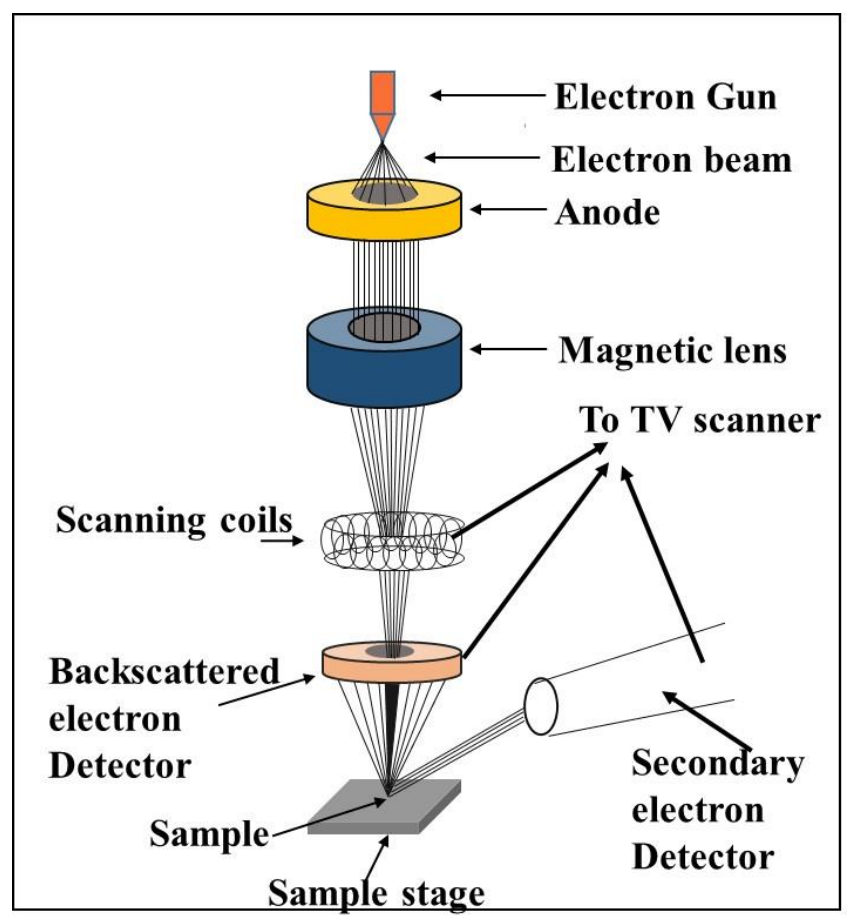

Fig. 2.8 Schematic of typical SEM chamber. After electron traverses through electromagnetic fields and lenses, it becomes high energy beam that interacts with the sample 
Whereas secondary electron detector uses in elastically scattered electrons from the sample surface and it gives topographical information. Back scattered electrons are more sensitive than secondary electrons. The X-ray radiation can be detected in the energy dispersive X-ray spectroscopy that can be used to identify specific elements in a sample [23]. A JEOL JSM 6700F SEM system was used for the dose optimization and to image the nanostructures. This system has a spatial resolution in the range of 50- $100 \mathrm{~nm}$.

\subsubsection{Magnetic force microscopy}

In this thesis, magnetic force microscopy (MFM) was extensively used to probe the magnetization configurations of in-plane magnetic nanostructures. MFM measurements were carried out on a Digital Instruments as shown in Fig. 2.9. The measurement system is shielded that isolate the system from the environment which may induce magnetic noise. The success of the MFM imaging relies on the separation of topological signal from the magnetic signal. To achieve this, MFM scanning is performed in the lift mode. A lift scan height of $50 \mathrm{~nm}$ was set for the MFM imaging, which ensures the separation of magnetic and topographical signals. In this technique, a tip coated with magnetic material is used to scan the surface of the sample to obtain the spatial variation of the magnetic field. As such, MFM image has lower spatial resolution compared to AFM measurement. MFM detects the force gradient between tip magnetization and the local magnetization of the sample surface [24-27]. The force gradient acting on the tip due to the stray field from the sample is given by

$$
\frac{\delta F}{\delta Z}=\int_{t i p}\left[m_{x} \frac{\partial^{2} H_{x}}{\partial^{2} Z^{2}}+m_{x} \frac{\partial^{2} H_{x}}{\partial^{2} Z^{2}}+m_{x} \frac{\partial^{2} H_{x}}{\partial^{2} Z^{2}}\right],
$$


where $F$ is the magnetic force, $Z$ is the component perpendicular to the sample surface, $m_{x}, m_{y}$, and $m_{z}$ are the magnetization components and $H_{x}, H_{y}$ and $H_{z}$ are the field components respectively.

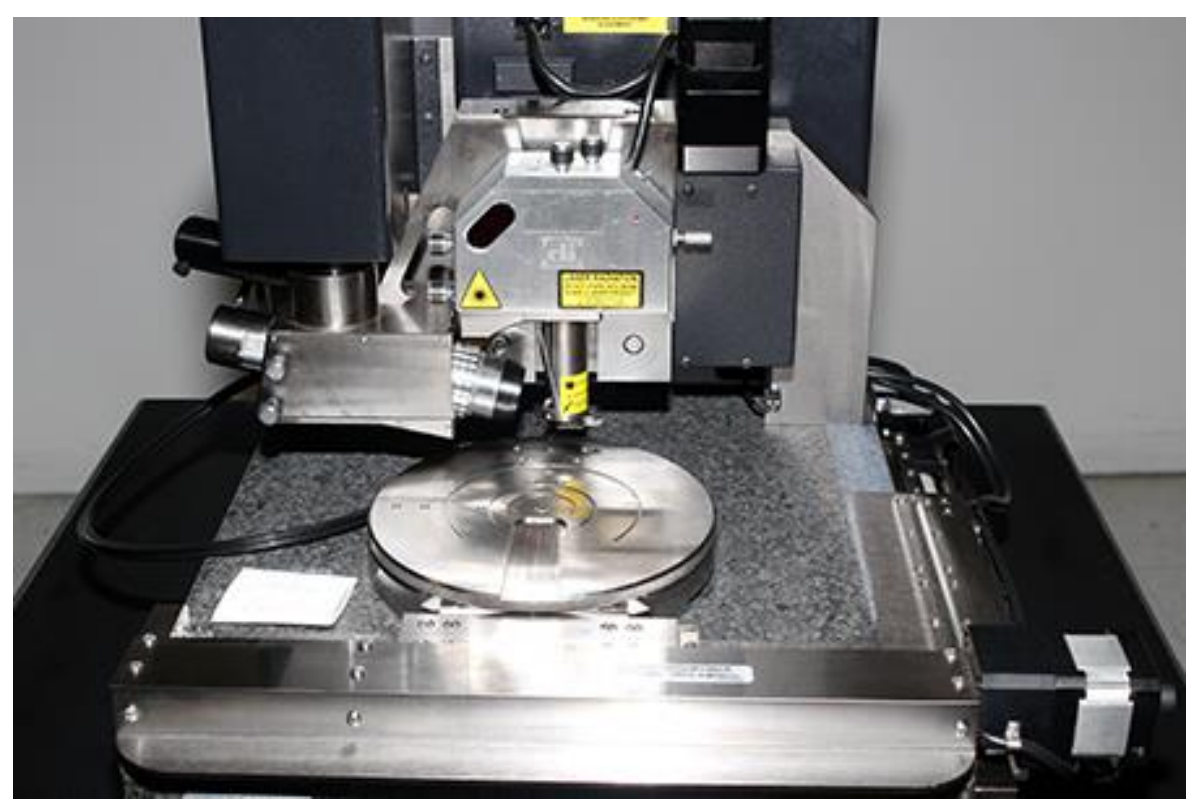

Fig. 2.9 Digital Instruments ${ }^{\circledR}$ scanning probe microscope. The system is enclosed in a cabinet which isolate the system from the noise.

Due to this force gradient, the tip vibrating at its resonance frequency suffers a shift in the frequency. The measured shift in the frequency can be correlated to the force gradient by

$$
\frac{\delta F}{\delta Z}=-2 K \frac{\delta f}{f},
$$

where $K$ is the spring constant of the tip, $f$ is the resonant frequency of the tip. The frequency shift $\delta f$ causes the phase difference which is used for MFM imaging. Fig. 2.10 shows the MFM imaging of a flux closure domains. 
The major problem with the MFM imaging is the interaction between the tip and the sample, which is large enough to affect the magnetization of the sample. The stray field from the tip can cause distortion in the DW structures. In some cases, it drags the DW along the nanowire.

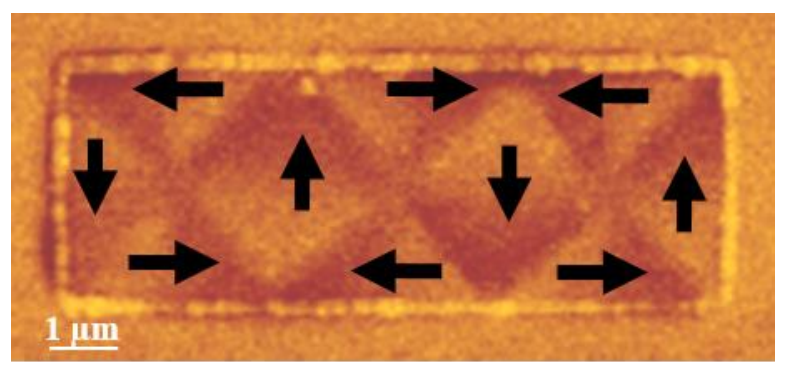

Fig. 2.10 The MFM image of flux closure pattern in $\mathrm{Ni}_{80} \mathrm{Fe}_{20}$ thin film.

It is very important to choose proper tip for imaging. For the measurements in this thesis, a low magnetic moment tip coated with $\mathrm{Si}-\mathrm{CoCr}$ was used. Due to the magnetic coating, the spatial resolution of the MFM tip lower comparted to the AFM tip. The MFM tip used for the measurements in the thesis has a resolution down to $25 \mathrm{~nm}$.

\subsubsection{Probe station measurement setup}

All of the DW generation and propagation measurements were performed on a commercial probe-station [28] equipped with two home made electromagnets that generate both in-plane field of $\sim 1 \mathrm{kOe}$ and out-of-plane field of $\sim 3 \mathrm{kOe}$. Fig. 2.11 shows the snapshot image of the probe station set up for electrical measurements.

High frequency RF probes which have a band width of $40 \mathrm{GHz}$ are used for DW injection and driving while the DC probes which have a band width of $5 \mathrm{GHz}$ are used to measure the resistance of nanowire. Resistance of the device was measured by two-point 
probe technique. A DC voltmeter and DC current source meter are connected in parallel to measure the resistance.

(c)
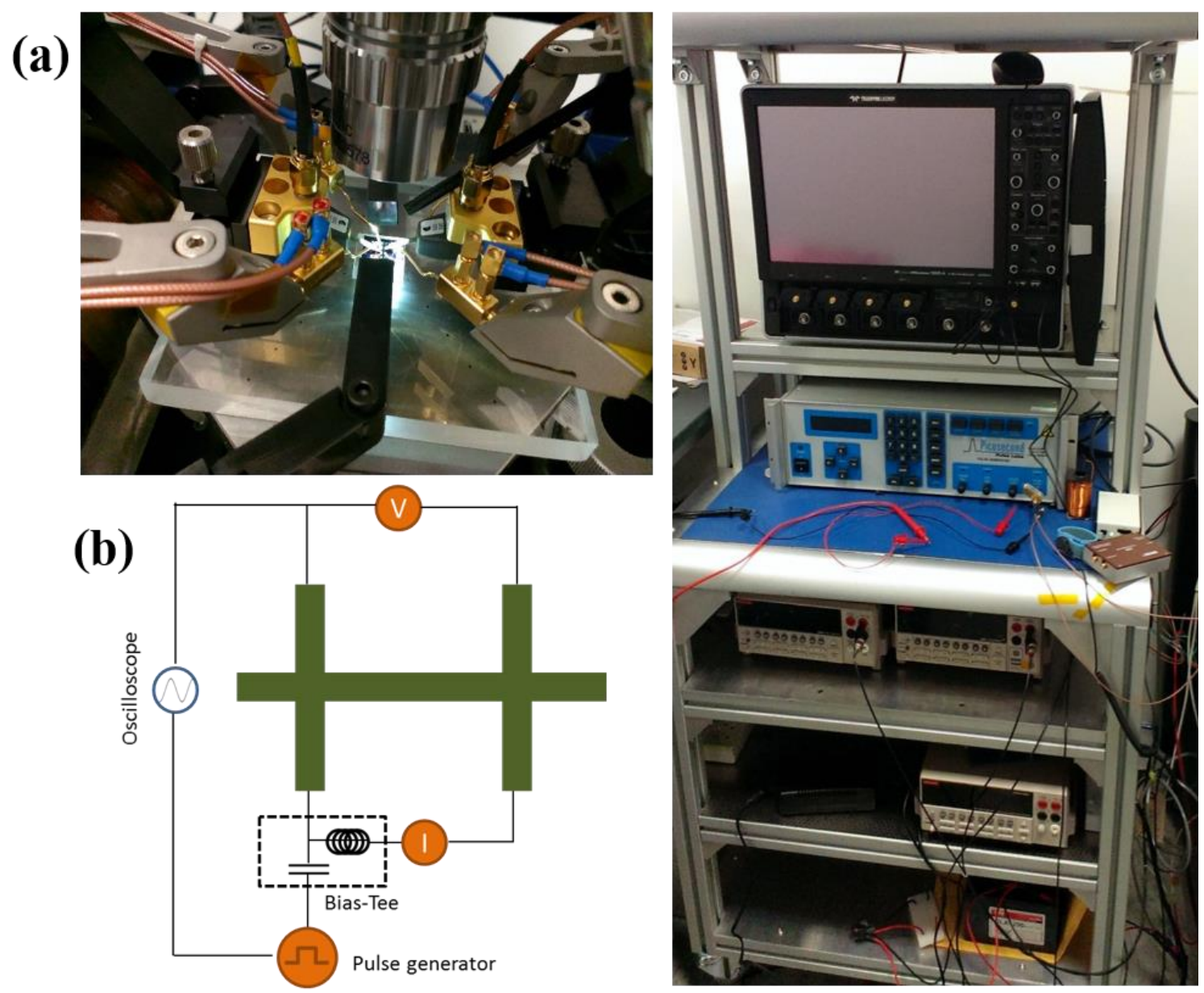

Fig. 2.11 Snapshot image of the setup for performing electrical measurements. (a) RF probes, DC probes and an in-plane electromagnet. (b) Schematic of the measurement setup (c) A cabinet which includes from top are Oscilloscope, Picosecond pulse generator (Picosecond Pulse Labs 10300B), a bias tee, DC voltmeter and current source.

A pulse generator (Picosecond Pulse Labs 10300B) was employed for injecting current pulses for DW injection and driving. A Bias tee was used to separate the DC source from the pulse generator. 


\subsubsection{Domain wall generation}

For DW generation, local Oersted field generation method was employed [29]. Fig. 2.12 (a) shows the schematic of a nanodevice with a measurement setup for the generation of DW. One end of the strip line (A) is connected to a constant voltage pulse generator and the other end is connected to a ground to avoid any multiple reflection of the injected pulse. Fig. 2.12 (b) shows a current pulse obtained from the other end of the injection line via Oscilloscope. A voltage pulse can be regarded as the current pulse as long as the resistance of the strip line does not show any appreciable change.

(a)

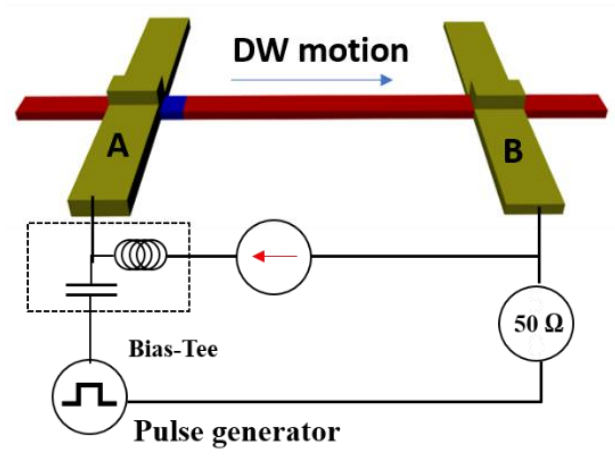

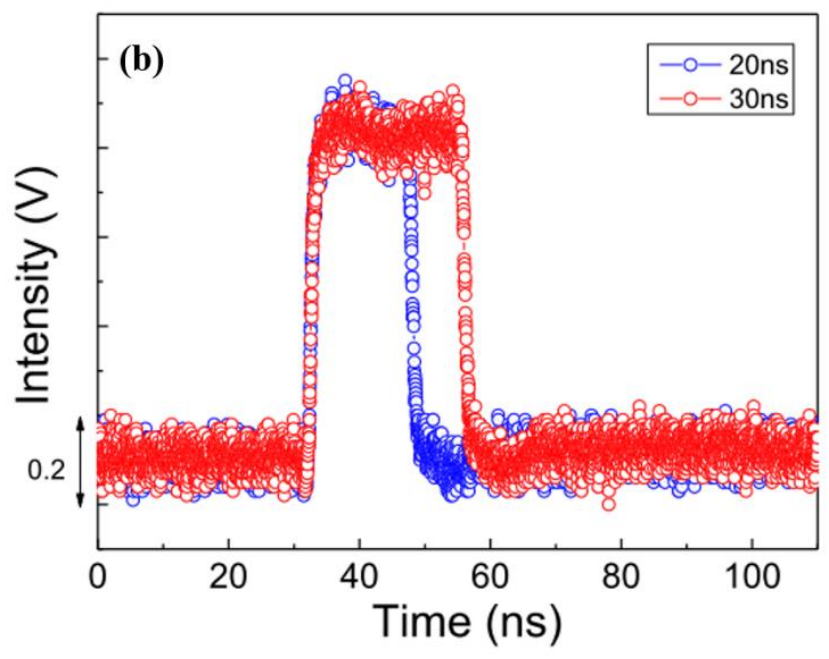

Fig. 2.12 (a) Schematic of the measurement setup for the DW injection. DW is injected via local Oersted field method. (b) In this method, current pulses are applied along the injection strip line.

When current pulse is injected through the strip line, an Oersted field is generated around the strip line, which reverses the local magnetization underneath the strip line. The 
presence of the DW in in-plane nanowires can be detected by the drop-in resistance, due to AMR effect while the same can be detected by AHE for out-of-plane nanowires.

\subsubsection{Domain wall driving setup}

After injection of the DW, it can be driven along the NW by applying field or current. Fig. 2.13 shows the schematic of the measurement setup for current induced DW driving. For current induced DW motion, series of multiple pulses, each with $50 \mathrm{ns,}$ are injected, which prevents the effect of joule heating. The pulses are injected from B to A into the nanowire. The resistance is measured after each series of pulses to ensure the DW movement.

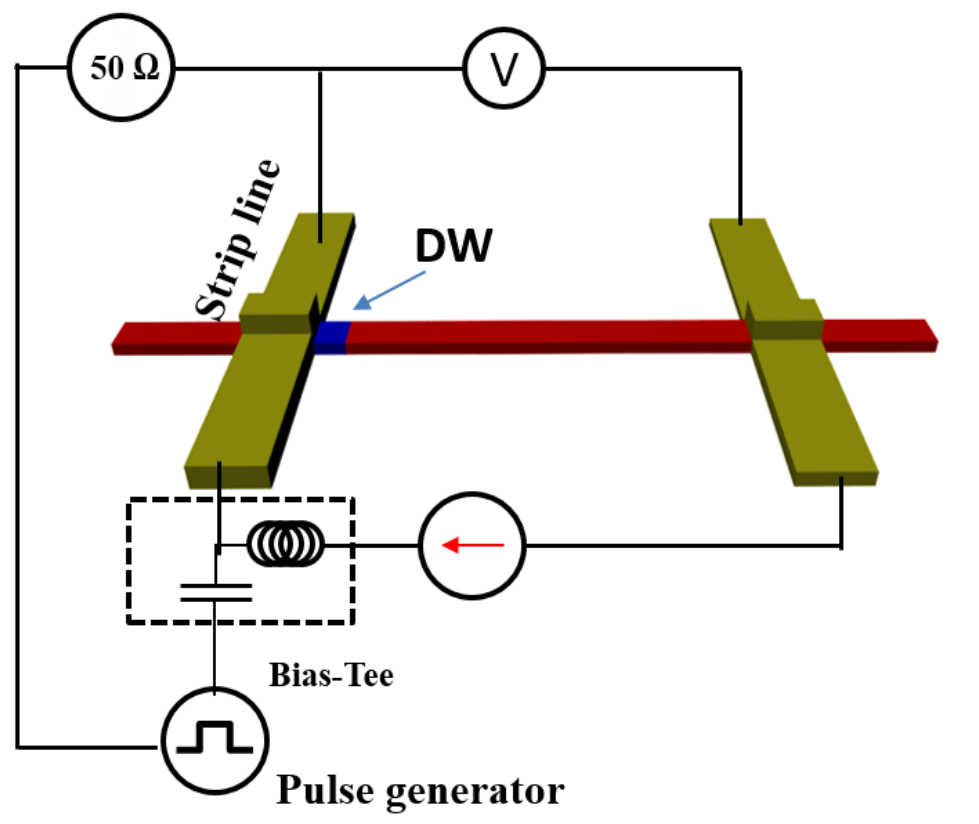

Fig. 2.13 Schematic of the measurement setup for DW driving. For DW driving, nanosecond pulses are feed into the nanowire by pulse generator. Then the DW can be moved either by STT or SOT. 


\subsubsection{Method to measure DMI strength and sign}

Current induced spin-orbit torques (SOT) in ultrathin layers with perpendicular magnetic anisotropy material have been extensively investigated due to its highly efficient magnetization switching and fast domain wall motion [30-40]. SOT drives domain wall opposite to the electron flow direction, which is contradictory to the prediction of conventional spin transfer torque effect (STT) [41-45]. Such behavior can only be explained by a combination of SOTs, from either Rashba or spin Hall effect, with a specific domain wall configuration [46-48]. It has been recently proposed that the DzyaloshinskiiMoriya interaction (DMI) is responsible for such domain wall configuration. DMI is an antisymmetric exchange interaction in ferromagnetic system arises from broken inversion symmetry and strong spin-orbit coupling. DMI induces an effective field (HDMI) which further stabilizes Néel domain wall [49-51]. The presence of DMI can be inferred from an in-plane assissted out-of-plane field induced domain expansion [52-53]. As this method uses only magnetic field to induce domain expansion, it removes any artifacts caused by current induced effects such as spin Hall effect and Rashba effect.

Fig. 2.14 (a) shows schematic of Kerr microscope setup used for measuring DMI. It is equipped with two home-made electromagnets which generate both in-plane and out-ofplane fields. An out-of-plane field is used to induce domain expansion while an in-plane field breaks the inversion symmetry. As shown in Fig. 2.14 (b), in the absence of in-plane field $\left(H_{x}=0\right)$, the domain expansion is symmetric with respect to an axis parallel to out-ofplane field. However, when an in-plane field is applied in conjunction with out-of-plane field, the domain expansion becomes asymmetric as shown in Fig. 2.14 (b). It implies that the two DWs (Up/Down and Down/Up) move in opposite direction with different 
velocities. The asymmetry in the domain expansion is due to presence of $\mathrm{H}_{\mathrm{DMI}}$. $\mathrm{H}_{\mathrm{DMI}}$ aligns the internal magnetization directions of Up/Down and Down/Up domain walls in opposite directions. As such, the two domain walls moving in the opposite directions experience different effective fields $\left(\mathrm{H}_{\mathrm{x}}+\mathrm{H}_{\text {DMI }}\right.$ and $\left.\mathrm{H}_{\mathrm{X}}-\mathrm{H}_{\text {DMI }}\right)$, thus they move with different velocities.
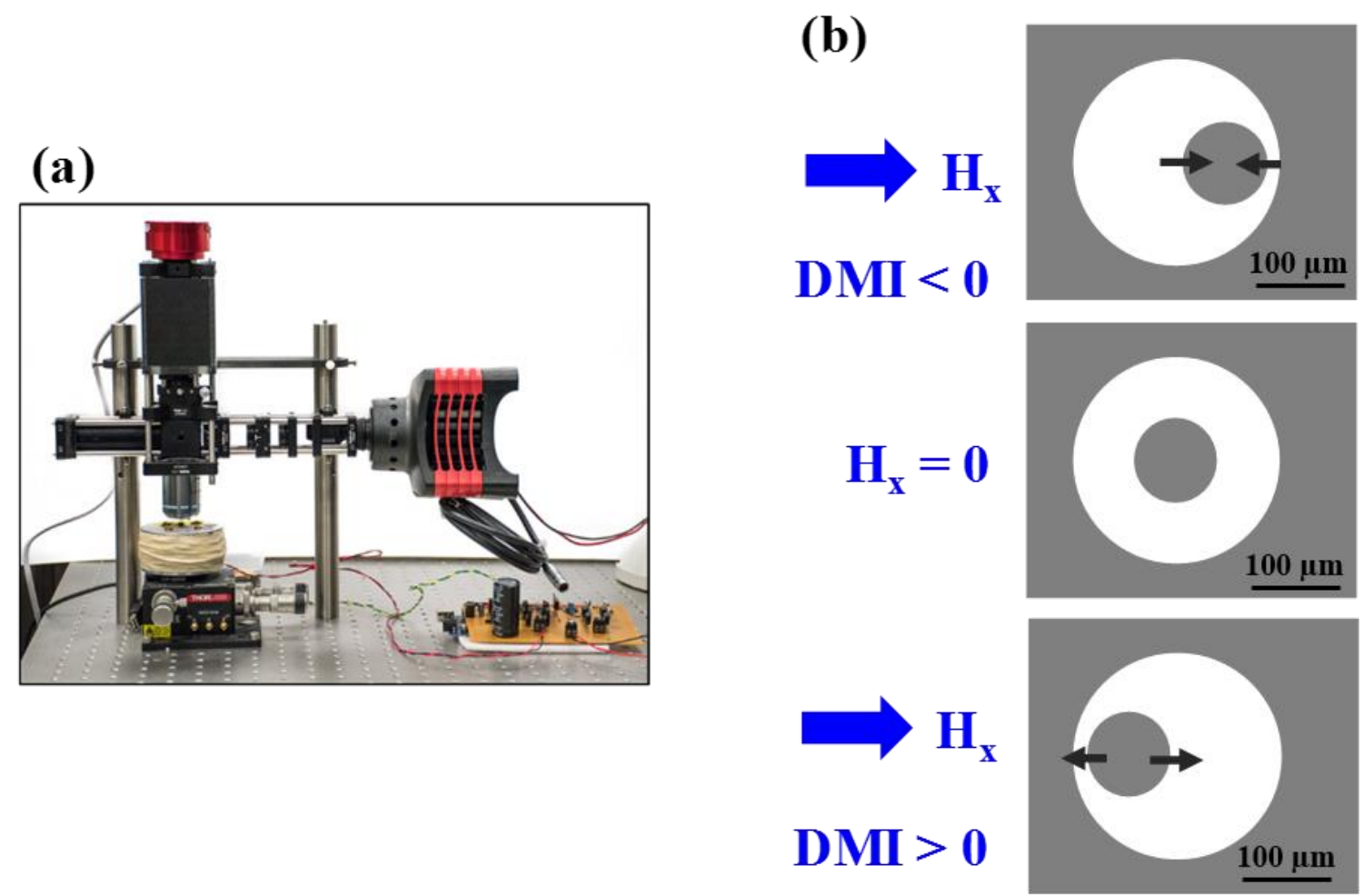

Fig. 2.14 (a) Schematic of Kerr microscope setup for DMI measurement. (b) Asymmetric domain expansion induced by simultaneous application of both in-plane field and out-ofplane field. Here white contrast represents Up magnetization.

The direction of asymmetry in the domain expansion determines the chirality of the Néel wall and hence the sign of the DMI. For a right-handed chiral Néel (DMI >0), Up magnetized domain (white contrast) expands asymmetrically towards positive direction while the opposite asymmetry is observed for left-handed chiral Néel wall. 


\subsubsection{Micromagnetic simulations}

To substantiate experimental results presented in this thesis, micromagnetic simulations have been performed. Micromagnetic simulations are indispensable tool for interpreting magnetization dynamics in ferromagnetic nanostructures. In the micromagnetic simulations, a ferromagnetic object is initially discretized into finite cells and then the LLG equation is solved in each cell. Two ways of discretization are commonly used; a finite difference method (FDM) where the space is discretized into identical cubic cells and finite element method (FEM) where discretization can take practically any shape [53]. The FEM has an advantage of employing less no. of cells but the cell size is sufficiently small in a region where the magnetizations configurations are abruptly changing. It results in lower computational speeds. On the other hand, the finite difference (FD) method uses more cells but it is faster in evaluating the dipolar fields arising from magnetization configuration. The dipolar interactions are the only contributions to the free energy of a ferromagnetic system, the computation of dipolar interactions takes most of the simulation time. Therefore run time of micromagnetic simulations relies on how fast the dipolar interactions can be computed. For a rectangular object with equally spaced mesh grid, the dipolar interactions can be can be written as a convolution which can be computed very effectively based on fast fourier transform method (FFT) [54]. This method has already been implemented on OOMMF micromagnetic simulations [55-56].

In this thesis, three different micromagnetic frame works were used; object oriented micromagnetic framework (OOMMF) developed by National Institute of Standards and Technology (NIST) [55], LLG 3D micromagnetic simulator [57] founded by M. R. 
Scheinfein. and Mumax by Vansteenkiste and Van de Wiele [58]. The three micromagnetic simulations work based on finite difference element method.

\subsubsection{OOMMF Micromagnetic simulations}

The OOMMF micromagnetic frame work is an open source software that generates numerical solutions of LLG eqn. (eqn. 1.17) in time and spatial domains. It replaces a continuum volume of a ferromagnetic sample with a discrete mesh of cells. Within each cell, the magnetization is considered to be uniform. So, each cell is associated with a fixed magnetic moment. Fig. 2.15 shows such a discretization.

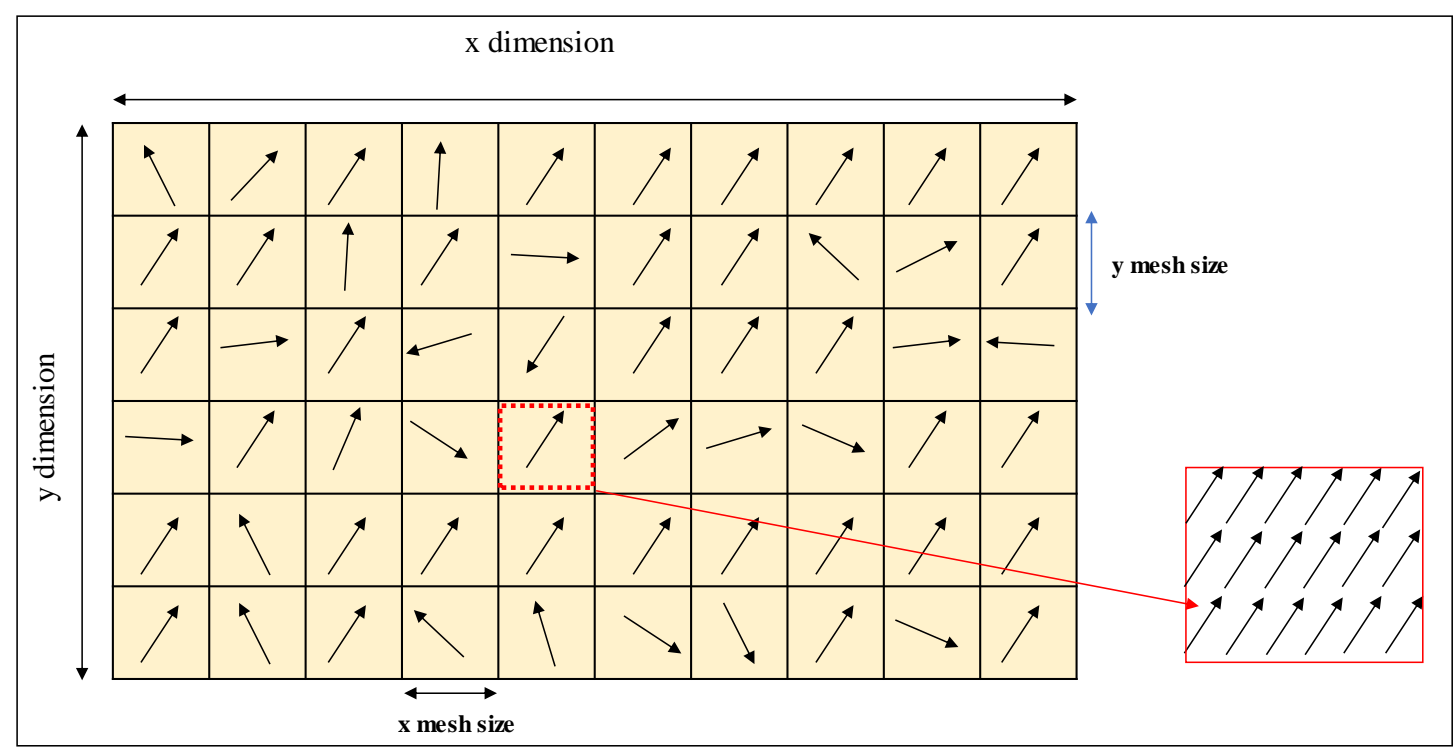

Fig. 2.15 Discretization into finite difference cubic cells. Within each cell the magnetization direction is considered to be uniform.

The accuracy of the simulation is determined by the cell size. When the cell size is comparable to the exchange length, the simulation provides accurate results. Across this length scale, the exchange energy is dominated and it align all spins parallel to each other. However, one cannot reduce the cell size beyond the limits of continuous 
approximation underlying the LLG equation. As such, OOMMF cannot be solve the problems where the cell size having the dimensions comparable to the lattice constant.

The OOMMF simulation software computes the equilibrium magnetization of each cell by iteratively solving Landau-Lifshitz equation (Chapter 1, eqn. 1.17) in each cell. It keeps iterating this computation until "stopping criteria" is reached. The stopping criteria depends on the amount of simulation time or the number of iterations. For steady state magnetization, the stropping criteria typically specified by "stopping_dm_dt=0, implying the evolver solves for the magnetization of a cell until the torque $d M / d t$ becomes zero. For magnetization dynamics, the stopping criterial can be set at any value between 0 to 1 .

The OOMMF software requires .mif and .bmp files as input files. The .mif file contains user written code which includes the device dimensions, cell dimension, magnetization saturation, exchange constant and magnetocrystalline anisotropy and field sequence. Whereas . bmp file provides information about the geometry of the structure and its projection in the $x y$-plane. OOMMF micromagnetic simulations have been used to study the spin state evolution of a transverse DW traverses through rectifier and inverter structures. It has also been used in investigating DW interaction in permalloy nanowires.

The standard material parameters of permalloy $\left(\mathrm{Ni}_{80} \mathrm{Fe}_{20}\right)$ used in the simulations are: saturation magnetization, $M_{s}=860 \times 10^{3} \mathrm{~A} / \mathrm{m}$, exchange constant, $\mathrm{A}=1.3 \times 10^{-11} \mathrm{~J} / \mathrm{m}$, anisotropy constant, $K=0$ and a damping constant $\alpha=0.01$. The nanowire dimensions were chosen to be $100 \mathrm{~nm} \times 10 \mathrm{~nm}$ so as to stabilize a transverse DW. To nucleate a transverse DW in the nanowire, the magnetization components are set as

$$
m_{x}=e^{\tanh \left(\frac{x-q}{\Delta}\right)}
$$




$$
\begin{aligned}
& m_{y}= \pm 0.1 \\
& m_{z}=0
\end{aligned}
$$

where $m_{y}=+0.1$ corresponds to a transverse DW with transverse component of magnetization along $+y$ direction while $m_{y}=-0.1$ corresponds to a transverse DW with transverse component of magnetization along $-y$ direction. Fig. 2.16 shows DWs with different transverse components of magnetization.

The output file provides two kinds of information: scalar quantities such as average magnetization, energies, torque, etc. and vector field display, which displays these quantities at each cell and gives a spatial map of the magnetization.

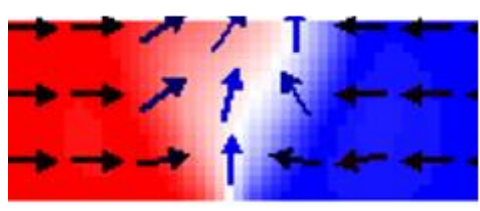

$$
m_{y}=+1
$$

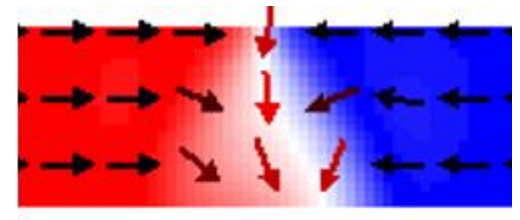

$$
m_{y}=-1
$$

Fig. 2.16 HH TDWs with transverse components of magnetization along $-y$ and $+y$ directions.

OOMMF micromagnetic simulations run at $0 \mathrm{~K}$ temperature. It neglects the thermal fluctuations and thermal noise. It may produce inconsistencies between the experimentally observed results and simulated results. In particular, thermal effects can influence the domain wall dynamics. 


\subsubsection{LLG micromagnetic simulator}

LLG micromagnetic simulator is a commercial software package founded by M. R. Scheinfein [57]. It solves Landau-Lifshitz-Gilbert equation using finite differences for exchange energies and boundary elements for magnetostatic energies. In this simulator, the structure is defined as a grid. LLG micromagnetic simulator has mainly three modules such as input phase, simulation phase and review phase.

The input phase, also known as data specification, is the central interface for coordinating input parameters, error checking and setting global parameters. Simulation phase is to set the graphical representation of the data. In the review phase, the graphical representation of the data can be viewed as it is essential to comprehending the results of a simulation. Fig. 2.17 shows the LLG micromagnetic simulator input phase.

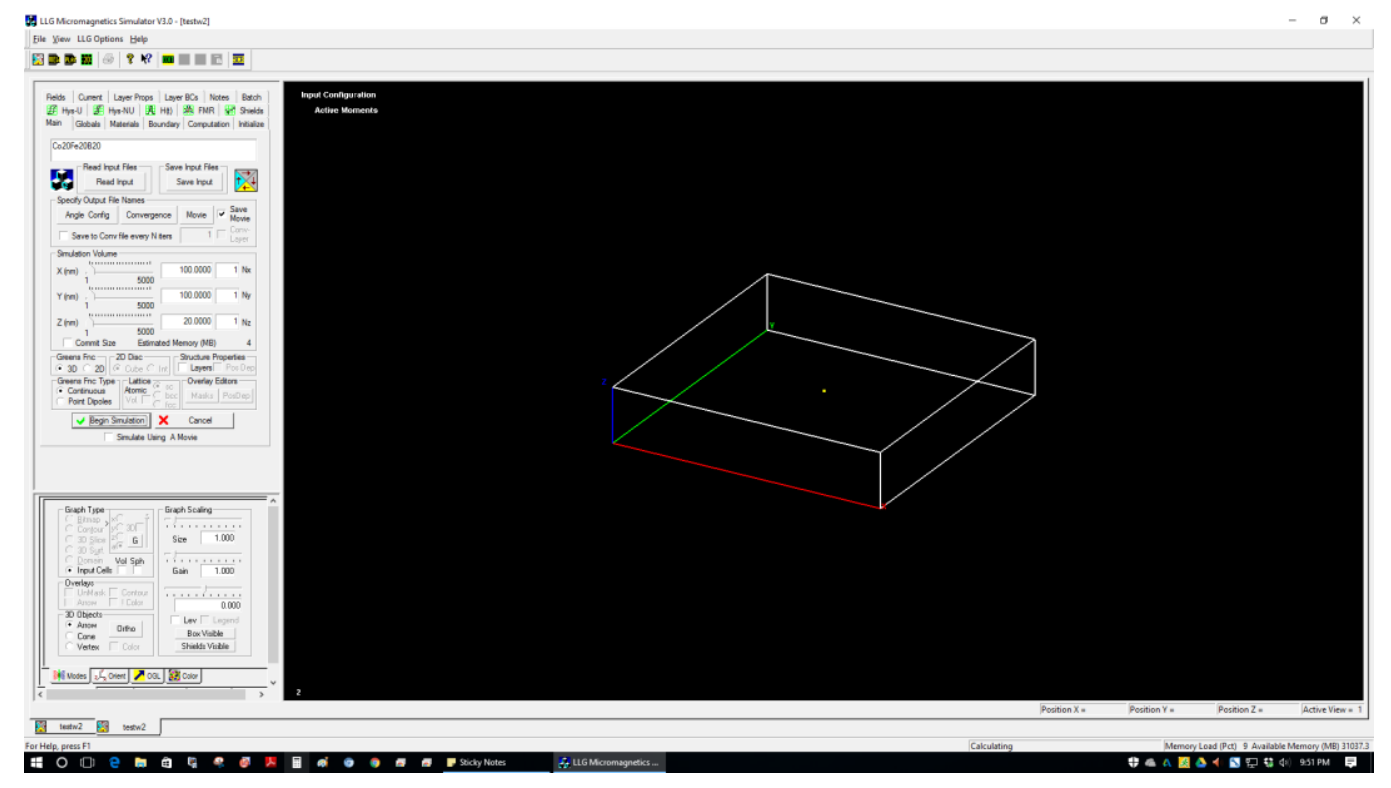

Fig. 2.17 LLG micromagnetic simulator input phase. The material parameters, current densities can be feed in this phase. 
OOMMF cannot simulate the structures where $3 \mathrm{D}$ currents are required to drive the DWs. Hence, LLG Micromagnetic simulator was employed to study the deterministic generation of single DW in permalloy nanowire. The standard material parameters for permalloy were employed.

\subsubsection{Mumax micromagnetic simulations}

Mumax micromagnetic frame work has been used to study chiral Néel wall dynamics driven by spin-orbit torque. Principally, Mumax is similar to OOMMF frame work, but it runs on Graphical Processing Units (GPUs) rather than Central Processing Units (CPUs) allowing high performance computations and large simulations. The computation speed of Mumax software is 100 times higher than the CPU based OOMMF software. It includes spin-orbit torques and Dzyaloshinskii-Moriya interactions which were used in the simulations.

In Mumax, 8 different Runge Kutta methods can be used to solve the LLG equation. In contrast to the OOMMF, finite temperature simulations can be performed using Mumax software. The input file for Mumax is written in Python which includes material parameters, dimensions of the structure, magnetic field sequence and current sequences. The input file for Mumax is similar to .mif file used in OOMMF simulations.

\subsection{Summary}

To summarize, magnetron sputtering was employed to deposit all the thin films studied in this thesis and electron beam lithography to pattern the nanostructures. The working principle of magnetron sputtering technique was discussed in detail. The key physics behind the structural characterization such as SEM was presented. The principles 
of magnetic imaging using MFM mode was discussed. Probe station was used extensively used to study the dynamic properties of DWs in both in-plane and out-of-plane magnetic nanowires. Therefore, the experimental setup for DW injection and DW driving are explained. Method to measure strength and sign of DMI in ultrathin layers of perpendicularly magnetized materials was finally discussed. 


\section{References}

1. P. D. Townsend, J. C. Kelly and N. Hartley, London: Academic Press, (1976).

2. R. Behrisch, In: Applied Physics, 47, (1981).

3. D. E. Harrison, Phys. Rev. 102, 1473 (1956).

4. H. Oechsner, Appl. Phys. A 8, 185 (1975).

5. S. Swann, Physics in Technology, 1967 (1988).

6. P. Kelly, R. Arnell, Vacuum, 56, 159 (2000).

7. S. S. Parkin, C. Kaiser, A. Panchula, P. M. Rice, B. Hughes, M. Samant and S. H. Yang, Nat. Mater. 3, 862 (2004).

8. R. J. Highmore, W. C. Shih, R. E. Somekh and J. E. Evetts, J. Mag. Mag. Mat 116, 249 (1992).

9. Please refer to http://www.ajaint.com/.

10. O. Wells, T. Everhart, R. Matta, IEEE Trans. Electron Devices ED12, 556 (1965).

11. T. H. P. Chang, Ph.D. thesis, Cambridge University, (1967).

12. A, Broers, W. Molzen, J. Cuomo, N. Wittels, Appl. Phys. Lett. 29, 596 (1976).

13. M. Isaacson, A. J. Murray, Vac. Sci. Technol. 19, 1117 (1981).

14. J. Yang, B. Cord, H. Duan, K. Berggren, J. Klingfus, S. Nam, K. Kim, M. Rooks, J. Vac. Sci. Technol., B 27, 2622 (2009).

15. B. Cord, J.Yang, H. Duan, D. Joy, J. Klingfus, K. Berggren, J. Vac. Sci. Technol., B 27, 2616 (2009).

16. H. Duan, V. Manfrinato, J. Yang, D. Winston, B. Cord, K. Berggren, J. Vac. Sci. Technol., B 28, C6H11 (2010). 
17. R. F. Pease, J. Vac. Sci. Technol., B 28, C6A1 (2010).

18. R. Howard, H. Craighead, L. Jackel, P. Mankiewich, M. Feldman, Journal of Vacuum Science and Technology B 1, 1101 (1983).

19. Please refer to http://www.raith.com.

20. Z. L. Wang, J. Phys. Chem. B 104, 1153 (2000).

21. Z.L. Wang, P. Poncharal and W.A. de Heer, Proceedings of the carbon nanotubes by in situ TEM Microscopy and Microanalysis 6, 224 (2000).

22. J. I. Goldstein, D. E. Newbury, P. Echlin, D. C. Joy, A. D. Romig,C. E. Lyman, C. Fiori and E. Lifshin, Scanning Electron Microscopy and X-ray Microanalysis. 2nd ed, New York: Plenum Press. 820 (1992).

23. N. Hanada, E. Hirotoshi, T. Chikawa, E. Akiba and H. Fujii, J. Alloy Compd. 450, 395 (2008).

24. A. Hubert, W. Rave, and S. L. Tomlinson, Phys. Status solidi B 204, 817 (1997).

25. W. Rave, L. Belliard, M. Labrune, A. Thiaville and J. Miltat, IEEE Trans. Magn. 30, 4473 (1994).

26. J. M. Gracia, A. Thiaville, J. Miltat, J. Magn. Magn. Mater. 249, 163 (2002).

27. P. Grutter, D. Rugar, H. J. Mamin, Ultramicroscopy, 47, 393 (1992).

28. Please refer to https://www.cascademicrotech.com/

29. T. A. Moore, I. M. Miron, G. Gaudin, G. Serret, S. Auffret, B. Rodmacq, A. Schuhl, S. Pizzini, J. Vogel, and M. Bonfim, Appl. Phys. Lett. 93, 262504 (2008). 
30. I. M. Miron, P.-J. Zermatten, G. Gaudin, S. Auffret, B. Rodmacq, and A. Schuhl, Phys. Rev. Lett. 102, 137202 (2009).

31. I. M. Miron, G. Gaudin, S. Auffret, B. Rodmacq, A. Schuhl, S. Pizzini, J. Vogel, and P. Gambardella, Nat. Mater. 9, 230 (2010).

32. U. H. Pi, K. W. Kim, J. Y. Bae, S. C. Lee, Y. J. Cho, K. S. Kim, and S. Seo, Appl. Phys. Lett. 97, 162507 (2010).

33. I. M. Miron, T. A. Moore, H. Szambolics, L. D. Buda-Prejbeanu, S. Auffret, B. Rodmacq, S. Pizzini, J. Vogel, M. Bonfim, A. Schuhl, and G. Gaudin, Nat. Mater. 10, 419 (2011).

34. I. M. Miron, K. Garello, G. Gaudin, P.-J. Zermatten, M. V. Costache, S. Auffret, S. Bandiera, B. Rodmacq, A. Schuhl, and P. Gambardella, Nature (London) 476, 189 (2011).

35. L. Liu, O. J. Lee, T. J. Gudmundsen, D. C. Ralph, and R. A. Buhrman, Phys. Rev. Lett. 109, 096602 (2012).

36. L. Liu, C.-F. Pai, Y. Li, H. W. Tseng, D. C. Ralph, and R. A. Buhrman, Science 336, 555 (2012).

37. C.-F. Pai, L. Liu, Y. Li, H. W. Tseng, D. C. Ralph, and R. A. Buhrman, Appl. Phys. Lett. 101, 122404 (2012).

38. S. Emori, D. C. Bono, and G. S. D. Beach, Appl. Phys. Lett. 101, 042405 (2012). 
39. K.-S. Ryu, L. Thomas, S.-H. Yang, and S. S. P. Parkin, Appl. Phys. Express 5, 093006 (2012)

40. T. A. Moore, I. M. Miron, G. Gaudin, G. Serret, S. Auffret, B. Rodmacq, A. Schuhl, S. Pizzini, J. Vogel, and M. Bonfim, Appl. Phys. Lett. 95, 179902 (2009).

41. J.-C. Lee, K.-J. Kim, J. Ryu, K.-W. Moon, S.-J. Yun, G.-H. Gim, K.-S. Lee, K.-H. Shin, H.-W. Lee, and S.-B. Choe, Phys. Rev. Lett. 107, 067201 (2011).

42. D. C. Ralph, M. D. Stiles, arXiv, 0711.4608v3 (2009).

43. A. P. Malozemoff, J. C. Slonczewski, Magnetic Domain Walls in Bubble Material. (Academic Press, 1979).

44. S. Fukami, T. Suzuki, Y. Nakatani, N. Ishiwata, M. Yamanouchi, S. Ikeda, N. Kasai, and H. Ohno, Appl. Phys. Lett. 98, 082504 (2011).

45. A. Thiaville, S. Rohart, E. Jue, V. Cros, and A. Fert, Europhys. Lett. 100, $57002(2012)$.

46. S. Emori, U. Bauer, S. M. Ahn, E. Martinez, and G. S. D. Beach, Nat. Mater. 12, 611 (2013).

47. K. S. Ryu, L. Thomas, S. H. Yang, and S. S. Parkin, Nat. Nanotechnol. 8, 527 (2013).

48. G. Chen, J. Zhu, A. Quesada, J. Li, A. T. N'Diaye, Y. Huo, T. P. Ma, Y. Chen, H. Y. Kwon, C. Won, Z. Q. Qiu, A. K. Schmid, and Y. Z. Wu, Phys. Rev. Lett. 110, $177204(2013)$. 
49. K. S. Ryu, L. Thomas, S. H. Yang, and S. S. Parkin, Nat. Nanotechnol. 8, 527 (2013).

50. G. Chen, J. Zhu, A. Quesada, J. Li, A. T. N'Diaye, Y. Huo, T. P. Ma, Y. Chen, H. Y. Kwon, C. Won, Z. Q. Qiu, A. K. Schmid, and Y. Z. Wu, Phys. Rev. Lett. 110, 177204 (2013).

51. S. G. Je, D. H. Kim, S. C. Yoo, B. C. Min, K. J. Lee and S. B Choe, Phys. Rev. B 88, 214401 (2013).

52. A. Hrabec, N. A. Porter, A. Wells, M. J. Benitez, G. Burnell, S. McVitie, D. McGrouther, T. A. Moore and C. H. Marrows, Phys. Rev. B 90, 020402 (R) (2014)

53. F. Josef and T. Schrefl. J. Phys. D: Appl. Phys. 33, R135-R156 (2000).

54. M. Jones and J. J. Miles, J. Magn. Magn. Mater. 171, 190-208 (1997).

55. M. J. Donahue and D. G. Porter, “oommf-computer code, http://math. nist. gov/oommf,"

56. N. Hayashi, K. Saito and Y. Nakatani, Jpn. J. Appl. Phys. 35, 6065 (1996).

57. M. R. Scheinfein, LLG Micromagnetic Simulator, http://llgmicro.home.mindspring.com/

58. A. Vansteenkiste and B. Van de Wield, J. Magn. Magn. Mater. 323, 21 (2011). 


\section{Chapter 3}

\section{Transverse domain wall manipulation for logic applications}

\subsection{Introduction}

A novel concept towards magnetic logic, wherein the information can be encoded as the transverse profile of a domain wall (DW). The transverse profile of a DW can be considered as a data bit and it can be correlated to topological edge defects. In this chapter, a technique to control the topological defects of a transverse DW (TDW) was proposed and verified by using MFM measurement. This technique exploits controlled relaxation of a transverse DW within an angled rectangle. The relaxation process is found to be strongly dependent on the angle, $\theta$, with which the rectangle orients with respect to the long axis of the nanowire. Two regimes were observed: inversion and rectification. For $3<\theta<9$, the angle rectangle performs logic NOT (inversion). This implies that the output of the inverter is always complimentary to the input TDW. For $9<\theta<15$, the angled rectangle performs rectification of the TDW. A rectifier provides fixed output irrespective of the input TDW. The rectification/inversion operation was found to be observed for any type of TDW. The relaxation process of a TDW involves transformation of a TDW to a vortex domain wall within the structure and then transformed back to TDW at the exit of the structure. The operation of rectification/inversion is governed by the pinning/depinning of TDW at the modulation. Slight variations in the shape of the structure does not affect the rectification/inversion mechanism which explains the robustness of the device. The experimental results were substantiated with the micromagnetic simulations. 


\subsection{Motivation}

The propagation of DWs in ferromagnetic nanowire has been proposed towards making high-density non-volatile magnetic memory, logic devices and shift registers [1-6]. The success of these devices relies on precise control and manipulation of transverse domain wall (TDW) profile in nanowire conduits. Much research has been devoted to understand the DW dynamics in magnetic nanowires [7-11]. Changes in the TDW profile are crucial for device applications where the DW motion is strongly influenced by artificial pinning sites. Because the detailed spin configuration of a TDW can affect the nature and strength of the pinning potential [12-14]. A transverse DW can be described as a composite object comprises of elementary topological bulk and edge defects [15]. The topological defects are characterized by winding numbers. The interaction of two DWs with same winding number results in the formation of bound state while DWs with opposite winding numbers leads to annihilation [16-17]. For a bi-nanowire system, the coupling strength between two DWs is largely influenced by their edge defects [18-20]. Thus, the topological defects of TDW are very important for deterministic pinning and DW motion in complex network structures [21]. A lot of research has been carried out on geometrical structuring which allows for controlled DW structure. For instance, E. R. Lewis et al. used transverse nanowires arranged in a "comb like" structure which acts to prevent DW transformations thereby increasing the DW velocity [22-23]. The transverse nanowires also help to increase the fidelity length, beyond which walker breakdown occurs. However, for this structure, the magnetization of the nanowire has to be set prior to the DW injection. Further, A. Kunz et al., and M. T. Bryan et al. have shown that the perpendicular magnetic field can increased the DW speed [24-25]. The effect of transverse field is highly correlated to the chirality of 
the DW. Also, Burn et al. have focused on preserving the chirality of the DW rather than controlling the chirality [26]. All these works are focused on controlling the chirality of the transverse DW. Novel schemes for the controlling topological defects of TDW may pave the way for viable DW base spintronic devices.

\subsection{Topological manipulation of a transverse domain wall}

In this chapter, a new scheme to control the topological defects of a transverse DW was proposed. This scheme exploits controlled relaxation of the DW within an angled rectangle, where the rectangle makes an angle, $\alpha^{\mathrm{o}}$, with respect to the long axis of the nanowire. A systematic investigation of the dependence of angle $\alpha^{\circ}$ on the DW relaxation process was carried out via micromagnetic simulations. For this study, $\theta$ has been varied from 0 to $45^{\circ}$. For $0<\alpha^{0}<3$, DW does not undergoes any transformation within the angled rectangle and hence the output DW is always same as that of the input. Interestingly, for $3<$ $\alpha^{0}<9$, the structure performs Logical NOT (Inversion) operation. The inverter provides an output DW which is always complimentary to the input DW i.e. output DW with opposite edge defects. Further, for $10<\alpha^{0}<15$, the rectangle performs rectification of transverse DW. For the rectifier, regardless of the type of input TDW, the output has a fixed edge defects; $-1 / 2 \sim+1 / 2$. For $16<\alpha^{0}<45$, DW encounters a large potential barrier and DW undergoes structural deformation while traversing through the angled rectangle. Fig. 3.1 shows schematic of topological rectifier and inverter. The length of rectangle is twice the width of the rectangle. i.e. $l=480 \mathrm{~nm}$ and $b=240 \mathrm{~nm}$. The width of nanowire is $120 \mathrm{~nm}$ such that transverse DW is the stable DW configuration. 

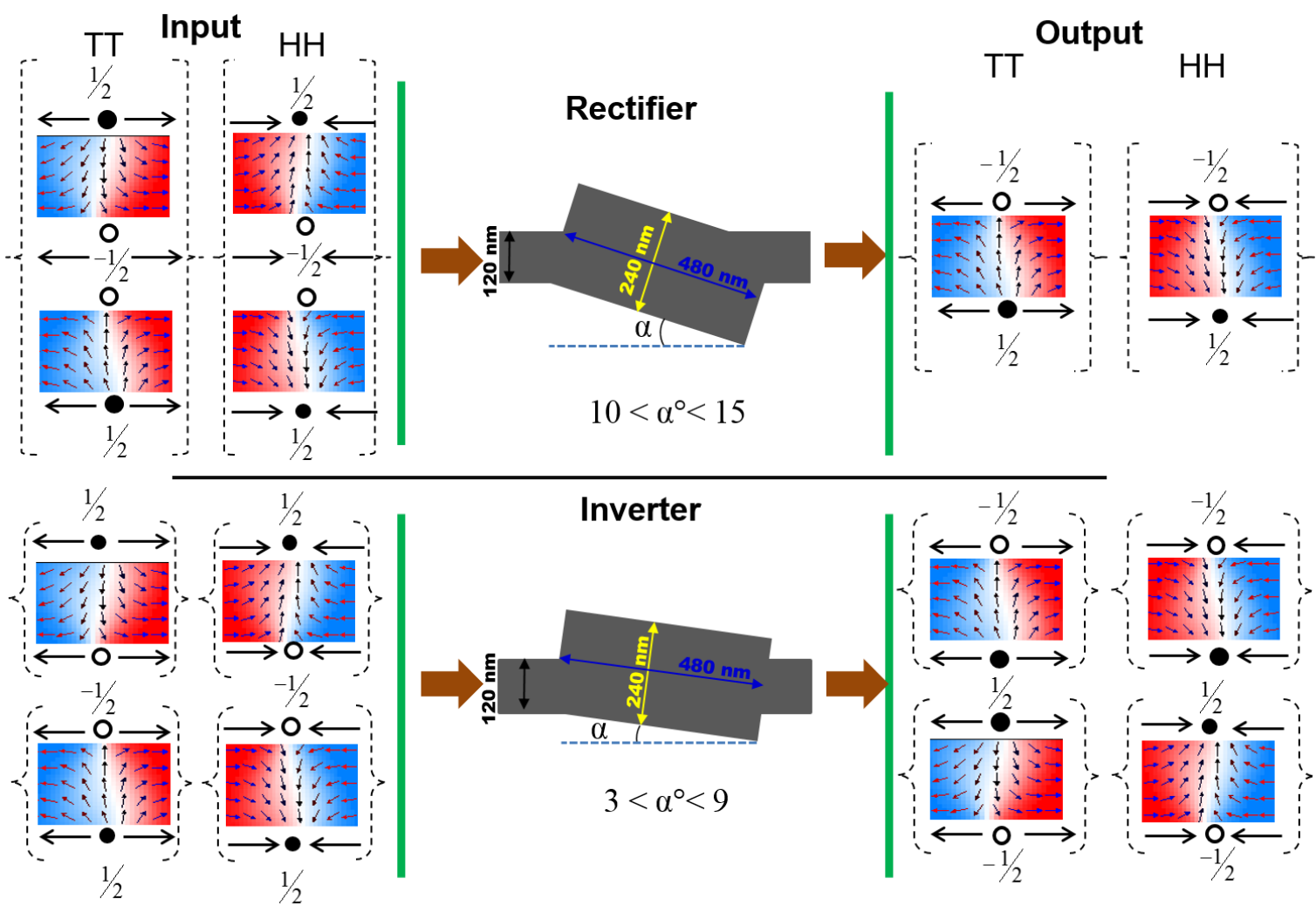

Fig. 3.1 Schematic representation of topological rectification and inversion of a transverse domain wall. For an inverter, where $3<\alpha^{\circ}<9$, the output has opposite edge defects as the input. For a rectifier, where $10<\alpha^{\circ}<15$, irrespective of the type of input DW the output always has fixed edge defects.

\subsubsection{Topological rectifier}

To gain an insight into spin details of a TDW propagating through a rectifier, object oriented micromagnetic framework (OOMMF) micromagnetic simulations have been carried out [27]. The standard material parameters of $\mathrm{Ni}_{80} \mathrm{Fe}_{20}$ were considered in the simulations: saturation magnetization $M_{s}=8.6 \times 105 \mathrm{~A} / \mathrm{m}$, damping constant $\alpha=0.01$, anisotropy constant $K=0$. The cell size of $5 \times 5 \times 5 \mathrm{~nm}^{3}$ was considered in the simulation. 
The edge defect for a tail-to-tail DW (TT DW) with transverse component lying along +y direction (TTU), is represented as $-1 / 2 \sim+1 / 2$ while for a TT DW with transverse component lying along $-\mathrm{y}$ direction (TTD), is described as $+1 / 2 \sim-1 / 2$. Fig. 3.2 shows the topological rectification of a TTD DW $(+1 / 2 \sim-1 / 2$ edge defects). For a TTD DW, the DW transforms from TTD to a vortex and subsequently to a TTU as it propagates through the rectifier. The spins at the entrance of the rectifier structure are aligned anti-parallel to the transverse component of the wall. The strength of the pinning potential of a TDW is largely influenced by the geometry of the potential barrier and the transversely varying energy profile of a DW. For a fixed geometry of the potential barrier, pinning is very effective when the highenergy component of the DW faces a potential barrier [28-32]. As the $+1 / 2$ edge defect has higher energy component [33-34], the TTD DW is pinned at the entrance of the rectifier. When the magnetic field is increased, DW depinning is via the nucleation of a vortex DW within the rectifier. The region where the transverse spins of a DW are pinned, results in a vortex core. The transverse component of a TTD DW determines the chirality of the vortex core. As the spins within the TTD DW are aligned along the $y$-direction, the left-hand side of the vortex core will follow this spin structure. As such, a vortex with anti-clockwise configuration is nucleated inside the rectifier structure. The transformation of the TDW into a vortex wall, allows it to overcome the potential barrier and move into the angled rectangle. Within the rectangle, the vortex core moves along $-y$ direction and subsequently reached to the lower edge of the rectifier structure. At the lower edge, the vortex core gets annihilated. As the vortex is in counter-clockwise direction, the spins on the right-hand side are oriented along $+y$-direction. Hence, the transverse component of the TDW exiting from the structure also points along + -y-direction i.e. TTU TDW. 

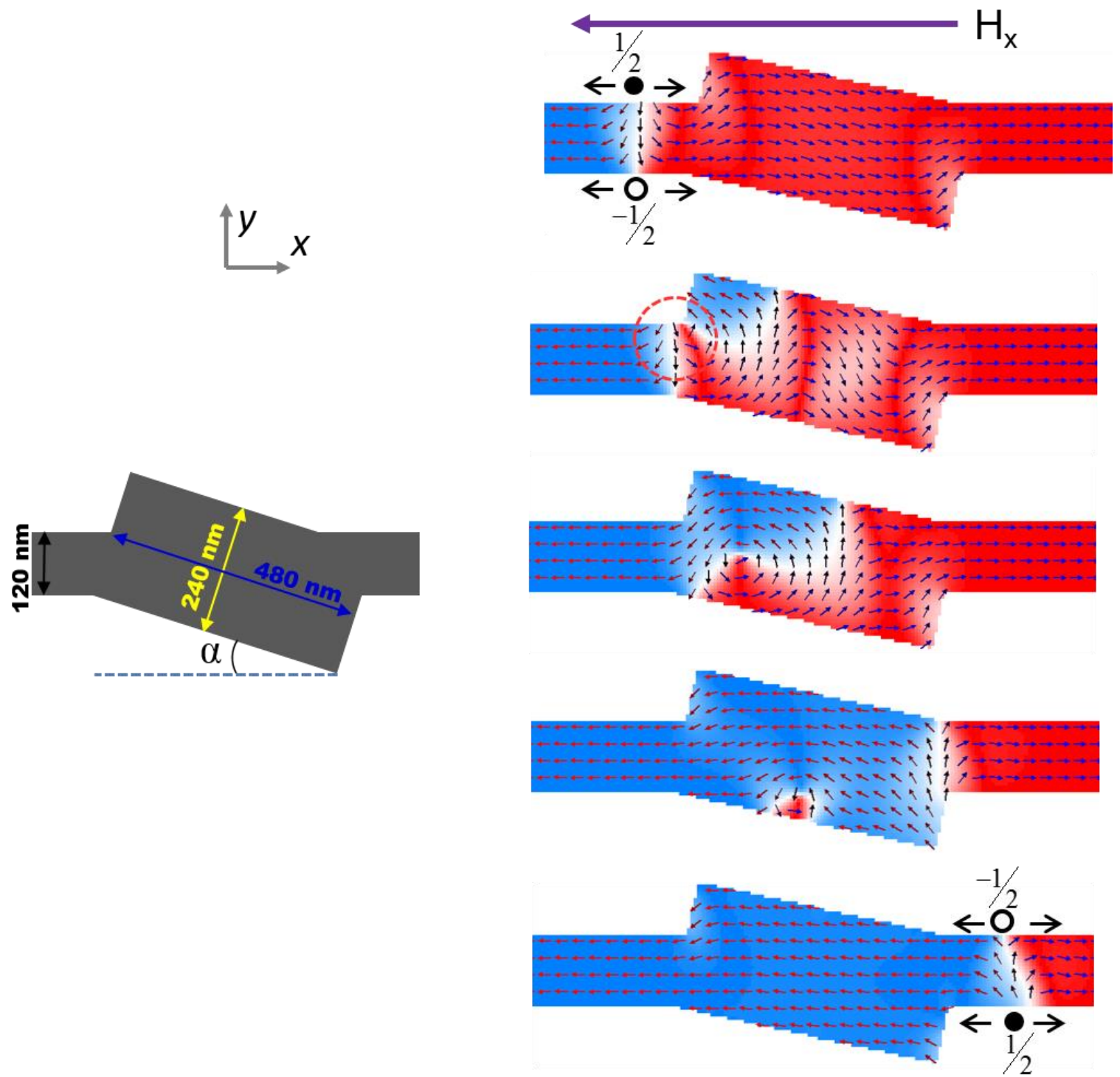

Fig. 3.2 Micromagnetic simulation results, which shows the spin configuration of a TDW as propagates through rectifier for TTD DW (+1/2 $-1 / 2)$. Domain wall undergoes controlled transformation from a TDW to vortex and back to a TDW with opposite edge defects. The schematic of the rectifier structure with dimensions are also shown.

The edge defects for this wall can be represented as $-1 / 2 \sim+1 / 2$. The field required for the TDW to perform this operation is $\sim 320$ Oe. As the DW further moves along the nanowire conduit, the strength of the applied field strength can be reduced such that effect 
of walker breakdown on the DW motion can be prevented. As such, the DW fidelity can be preserved. However, transformation of a TTU DW $(-1 / 2 \sim+1 / 2$ edge defects $)$ within the structure follows a different phenomenon. As shown in Fig. 3.3, both the transverse component of the wall as well as the spins at the right side of the structure align along the same direction.
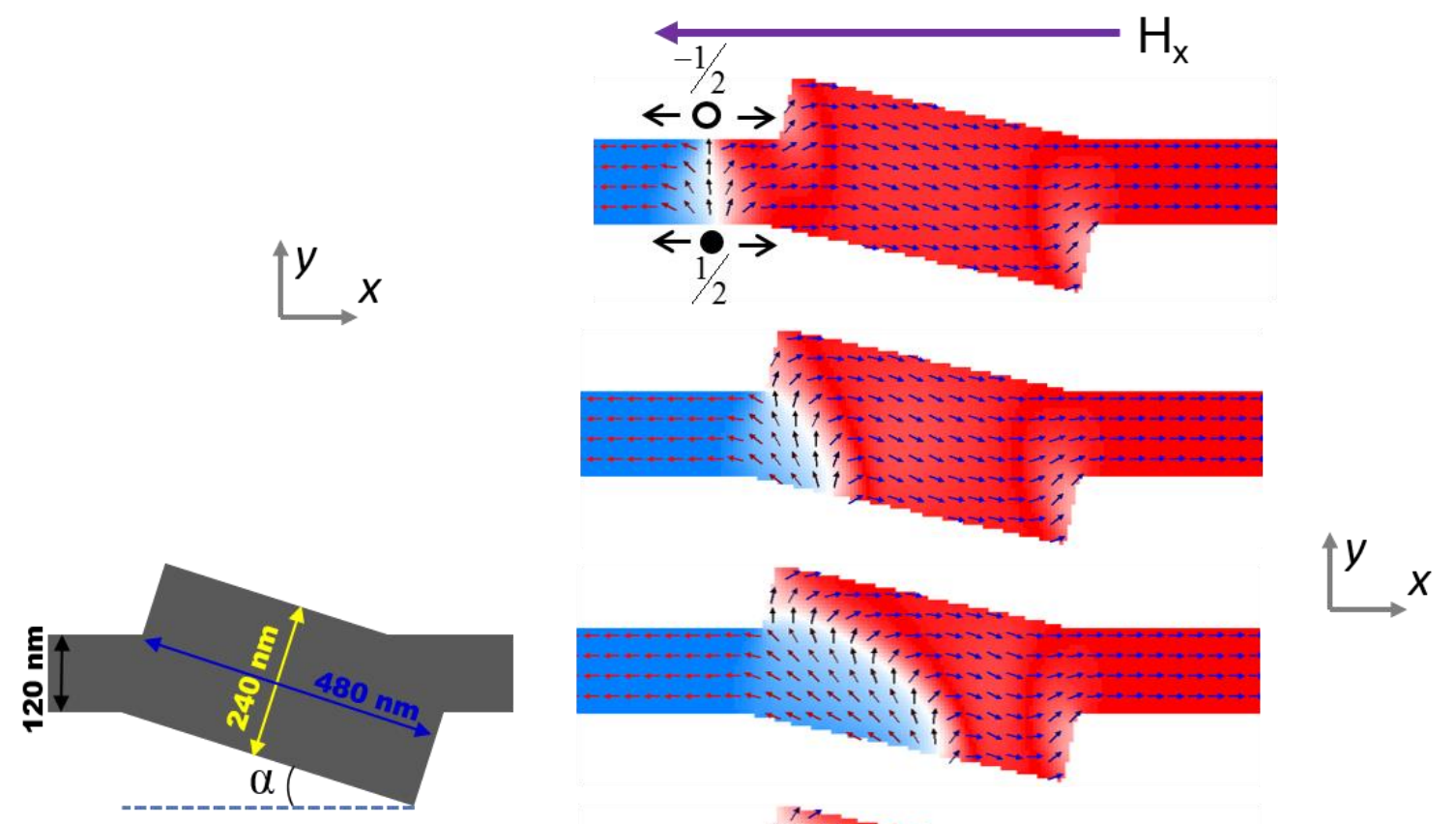

Fig. 3.3 Micromagnetic simulation results, where the spin state evolution of a TTD DW $(-1 / 2 \sim+1 / 2)$ traverses through structure rectifier structure. In this case, domain wall does not undergo any transformation during its propagation through the structure. As such, the output from the structure is same as that of the input. 
Additionally, the higher energy component of the wall $(+1 / 2$ winding number) is located on the bottom edge of nanowire, where the potential energy of the barrier is very low. As a result, the DW does not encounter any potential barrier while it enters into the structure. Up on increasing the field strength along $-x$ direction, the bottom edge defect $(+$ $1 / 2$ winding number) of the wall enters into the structure while the top edge defect $(-1 / 2$ winding number) pins at the upper edge of the rectifier structure. Further increasing field results in the bottom edge defect $(+1 / 2$ winding number) to move along the lower edge of the structure. It leads to the spins within the structure re-align along $+y$ direction. When the bottom edge defect of the wall $(+1 / 2$ winding number) reaches to the right edge of the structure, a DW with same spin configuration as that of input DW exits into the nanowire conduit. In essence, the rectifier always provides $-1 / 2 \sim+1 / 2$ edge defects as the output and hence it performs the topological rectification. As the topological defects for HH and TT DW are persistent, regardless of the transverse component within the wall, the rectifier performs topological rectification for any type of TDW (HH or TT). Additionally, by mirroring the structure along the nanowire axis, it has been observed that the rectifier always outputs $+1 / 2 \sim-1 / 2$. This is due to the fact that the spins within the structure influences the lower edge of the nanowire.

\subsubsection{Experimental verification of Rectifier}

A thin film stack of $\mathrm{Ta}(5 \mathrm{~nm}) / \mathrm{Ni}_{80} \mathrm{Fe}_{20}(10 \mathrm{~nm}) / \mathrm{Ta}(5 \mathrm{~nm})$ was deposited on $\mathrm{Si} / \mathrm{SiO}_{2}$ (300 nm) substrate by using DC magnetron sputtering. The bottom Ta layer is used as a seed layer while the top Ta layer prevents the underlying $\mathrm{Ni}_{80} \mathrm{Fe}_{20}$ from oxidation. The nanostructures were subsequently patterned using electron beam lithography and Ar ion milling. 
The magnetic force microscopy (MFM) in phase detection mode was used to probe the DW trajectory in the nanostructure. For MFM imaging, commercially available low magnetic moment tips coated with $\mathrm{CoCr}$ were used. This helps to minimize the effect of tip magnetization on the ferromagnetic material. For all the measurements, the tip was initially magnetized in such a way that the South pole is induced at its apex. An optimized lift scan height of $\sim 50 \mathrm{~nm}$ was used to remove the topography signal from the phase signal. All MFM images were captured in quasi-remnant state; after removing the external field.

\subsubsection{Domain wall injection}

In soft ferromagnetic materials $\left(\mathrm{Ni}_{80} \mathrm{Fe}_{20}\right)$, due to negligible crystalline anisotropy, the magnetization of the nanostructure is governed by its shape. For instance, in a permalloy nanowire, the shape anisotropy aligns the magnetization along the long axis of the wire. A magnetic DW can be injected into the nanowire by reversing the magnetization locally through the application of external magnetic field.

Various methods have been put forward to inject DW into a nanowire. For example, Lewis et al. proposed that the DW can be injected at the curvature of a L-shaped nanostructure by applying an oblique field [22-23, 35]. More recently, Guite et al. demonstrated a deterministic generation of a single DW by utilizing the internal stray field possessed by a magnetic nanowire [36]. Shigeto et al. proposed a simple method of DW injection by attaching a large nucleation pad to one end of the nanowire [37]. The magnetization of the nucleation pad switches at lower external field as compared to an isolated nanowire. The nucleation pad reverses the magnetization of the nanowire. The switching of the pad lead to the nucleation of a DW into the nanowire at wire/pad junction. This is due to the fact that the larger nucleation pad has lower coercivity. This technique 
was employed to inject DW in all of the devices investigated in this chapter. Fig. 3.4 shows SEM image of a nucleation pad fabricated on to the left end of the nanowire. The dimensions of the pad are $2 \mu \mathrm{m} \times 2 \mu \mathrm{m} \times 10 \mathrm{~nm}$.

The magnetization evolution of the nucleation pad during the magnetization reversal process was studied using MFM measurement. Initially, the structure is saturated by applying an in-plane field of $\sim 600$ Oe along $-x$ direction and then relaxed. Fig. 3.5 (a) shows the MFM image of magnetization configuration in the remnant state. When the magnetic field was increased to 70 Oe along $+x$ direction, the magnetization reversal initiates within the pad.

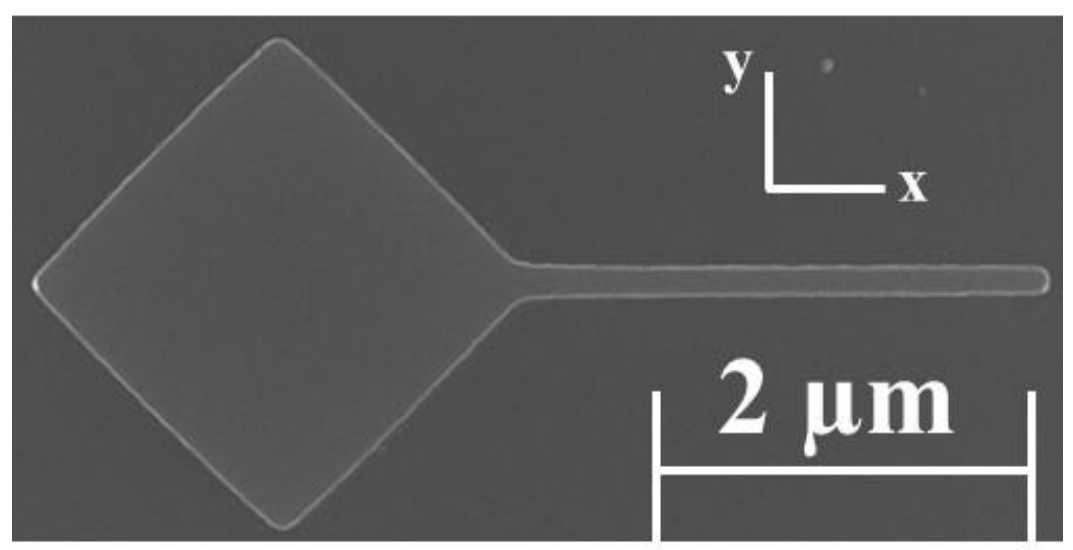

Fig. 3.4 SEM image of the nucleation pad together with a nanowire. The nucleation pad has dimensions of $2 \mu \mathrm{m} \times 2 \mu \mathrm{m}$ and nanowire has a width of $120 \mathrm{~nm}$.

As the magnetic field was further increased, a DW reaches at the junction of the $\mathrm{pad} /$ nanowire junction. Additional increase of the field results in the propagation of DW along the nanowire. Fig. 3.5 (b) shows the MFM image of the nucleation pad after the DW exits the nucleation pad. The bright contrast on opposite sides of the nucleation pad represents the opposite magnetization directions of the nucleation pad. As such, this field 
sequence injects a head-to-head (HH) DW into the nanowire. Similarly, a tail-to-tail (TT) DW can also be injected by first saturating the nanostructure by applying field along $+x$ direction and subsequently along $-x$ direction to nucleate the DW. This method has an advantage of injecting HH or TT DW deterministically, but the chirality of the TDW cannot be controlled.
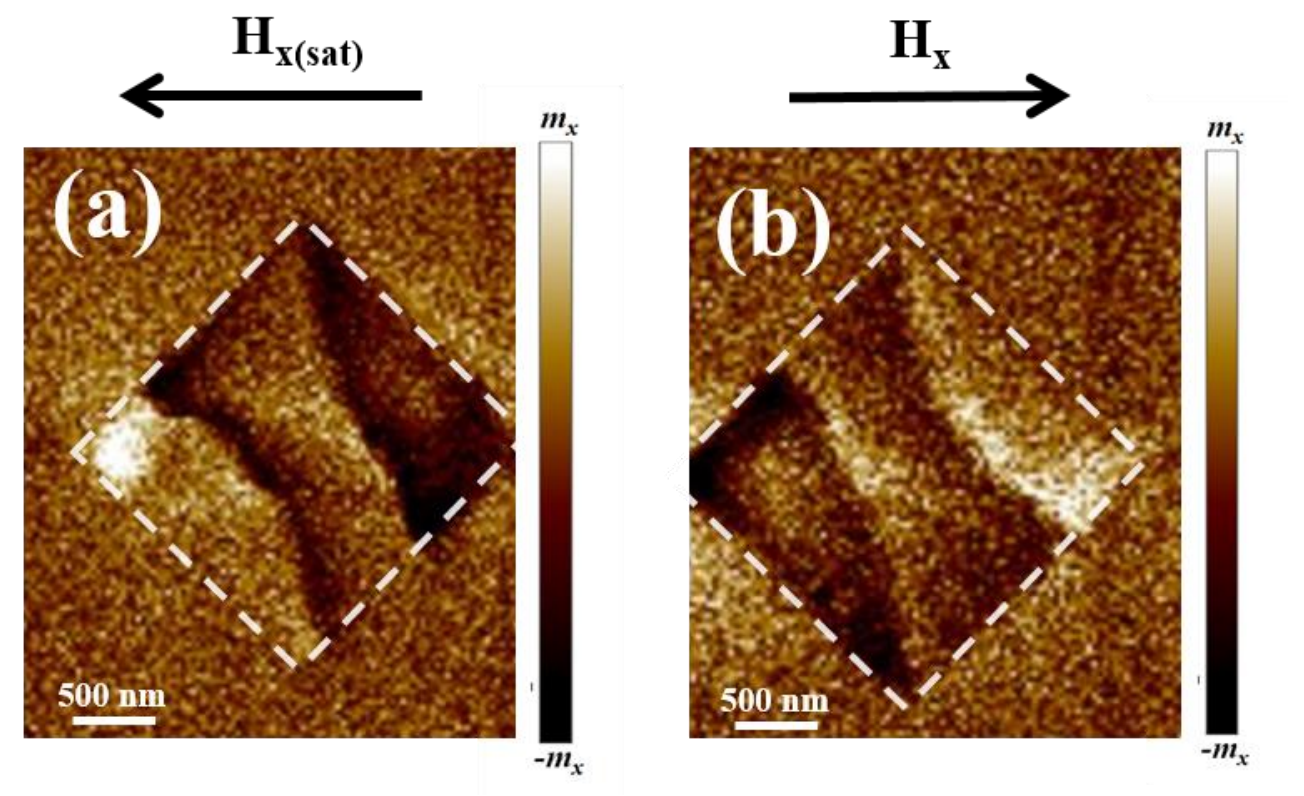

Fig. 3.5 The magnetization evolution of the nucleation pad during DW nucleation. (a) Magnetization configuration in the remnant state obtained by saturating the structure by applying large in-plane field along -x direction. (b) Magnetization reversal under the field of 70 Oe applied along $+x$ direction.

\subsubsection{Domain wall chirality control}

To control the chirality of the TDW being injected, a transverse wire is placed at the junction between the nucleation pad and nanowire. Fig. 3.6 shows the SEM image of a $5 \mu \mathrm{m}$ long and $200 \mathrm{~nm}$ wide chirality selector. Prior to the DW nucleation, the chirality control is saturated either in $+(-) y$ direction by applying a large transverse magnetic field. 
The chirality selector then induces the transverse component of the nucleated DW to align along a specific magnetization direction. The magnetization of the chirality selector, due to strong shape anisotropy, is not altered by the field sequences used for the device saturation and DW nucleation.

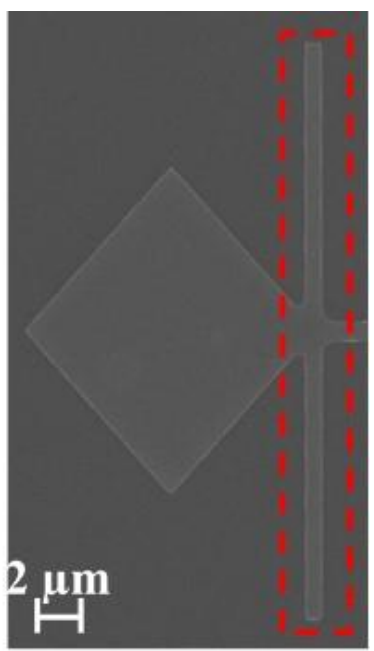

Fig. 3.6 SEM image of the chirality selector (red dotted line) connected to NW on one side and nucleation pad on the other side. The nucleation pad injects DWs into the nanowire because the nucleation pad has lower switching field compared to the nanowire.

\subsubsection{Topological Detector}

There is no available tool to directly probe the topological defects of a TDW. Sophisticated techniques such as electron holography transmission or X-ray microscopy technique can only probe internal spin structure of TDW [33-34, 38]. Hayashi et al. demonstrated that the AMR measurement can be used to detect the type of TDW [39]. However, the difference in the AMR signals for various types of TDW is significantly low.

The experimental verification of rectifier and inverter was carried out by exploiting the chirality dependent motion of a TDW in branch structure. It has been demonstrated that 
the trajectory of a vortex DW in Y-shaped structure can be controlled by the topological defects [21]. More recently, it was shown that the TDW moving through a Y-structure also follows the same principle [40]. Initially, this principle was first verified experimentally to ensure that different TDW configurations can be reliably detected.

Fig. 3.7 shows SEM image of a topological detector whose dimensions are the same as rectifier/inverter dimensions. It comprises of a nucleation pad, a chirality selector and a nanowire.

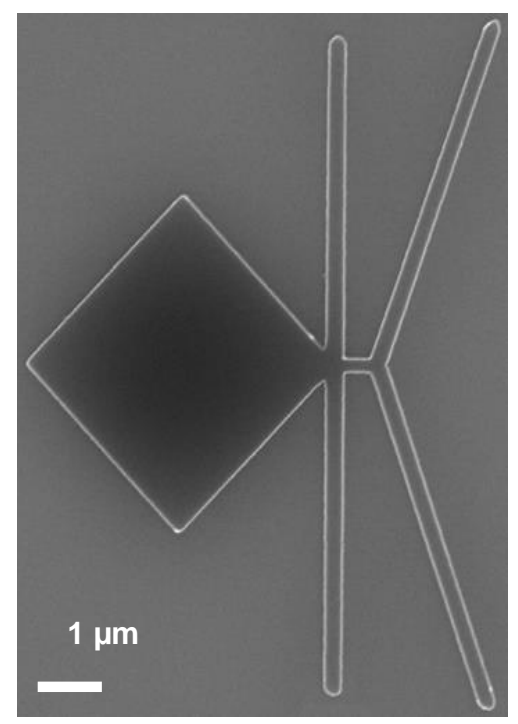

Fig. 3.7 SEM image of the TDW detector. A large nucleation pad on the left side is used to nucleate DW in the NW. A transverse nanowire acts as chirality selector. $Y$-shape structure at the end acts a detector.

The nanowire has a width of $120 \mathrm{~nm}$ which ensures the TDW is a stable configuration. The Y-shape detector consists of two wires angled at $\sim \pm 70^{\circ}$ with respect to the $x$-axis. The DW detector and chirality selector have a width of $120 \mathrm{~nm}$. The spacing between the chirality selector and detector is kept fixed at $200 \mathrm{~nm}$. 
The topological detector was first verified to ensure that different types of TDWs can be accurately detected. To accomplish this, a systematic MFM measurement has been carried out. In order to create a DW with specific topological defects $[+1 / 2 \sim-1 / 2$ or $-1 / 2 \sim$ $+1 / 2]$, the chirality selector was first saturated by either in $+(-) y$ direction by applying a large magnetic field of $\sim 500$ Oe. The nanostructure was subsequently saturated along either $-(+) x$ direction by using a longitudinal field of $\sim 500$ Oe. Fig. 3.8 (a) shows the initial configuration of the detector.
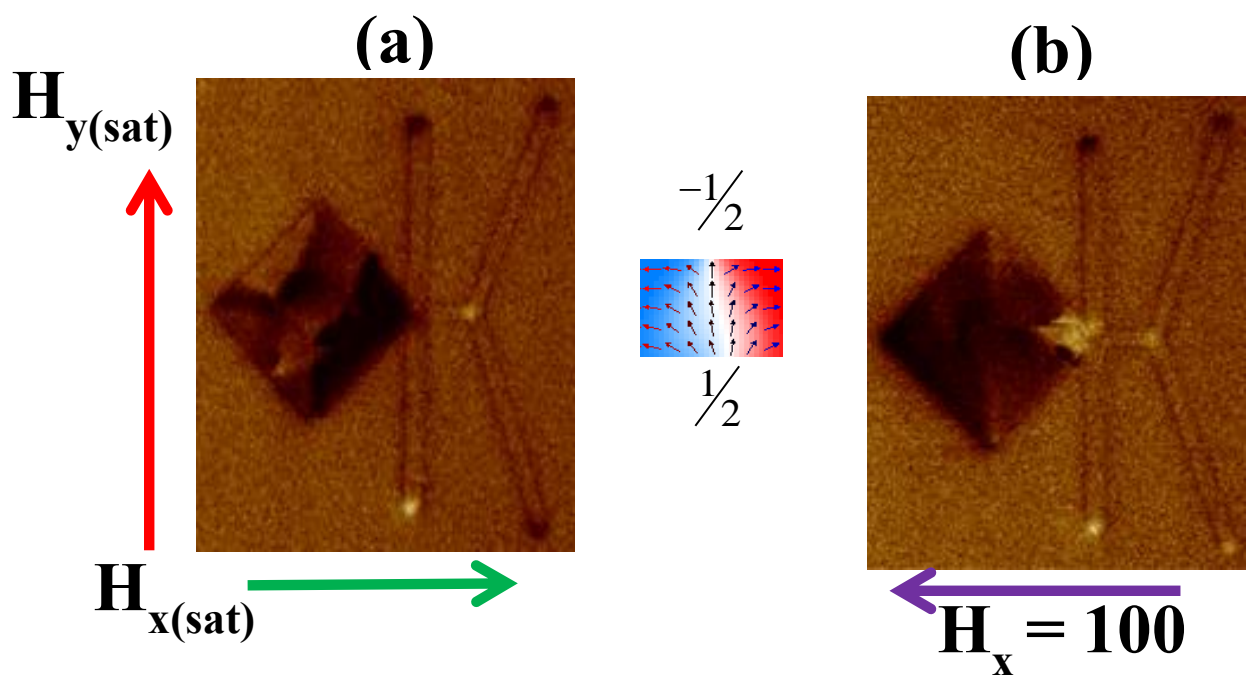

Fig. 3.8 Initial (a) and final (b) magnetization states of the nanostructure when $a-1 / 2 \sim+1 / 2$ DW injected and propagated in the NW. Chirality selector is magnetized by applying a large transverse field $\left(-H_{y}\right)$ while the nucleation pad and the nanowire are saturated by applying a longitudinal field $\left(+H_{x}\right)$.

One can clearly see bright (south pole) and dark (north pole) contrasts on either side of the chirality selector, implying the chirality selector is saturated along the $+y$ orientation while the device is saturated along $+x$ direction as evident by the dark contrast at the two ends of branch structure. 
A field of 50 Oe was found to be enough for the DW to nucleate and move it to the branch structure. An additional field strength of 100 Oe results in the propagation of the DW towards the end of the Y-shaped detector. Fig. 3.8 (b) shows the representative configuration of the nanostructure. It signifies that the magnetization direction of the lower branch of the Y-shaped detector has been changed as evident from the bright contrast at the lower part of the detector. As such, a TDW with $-1 / 2 \sim+1 / 2$ edge defects is indeed injected and moved towards the bifurcation of the detector. The interaction of the TDW with the bifurcation results in a TDW moving into the lower branch of the Y-shaped detector.

Similarly, the chirality selector is magnetized along $-y$ while the device along $+x$ direction. The representative magnetization configuration of the device can be seen from Fig. 3.9 (a).

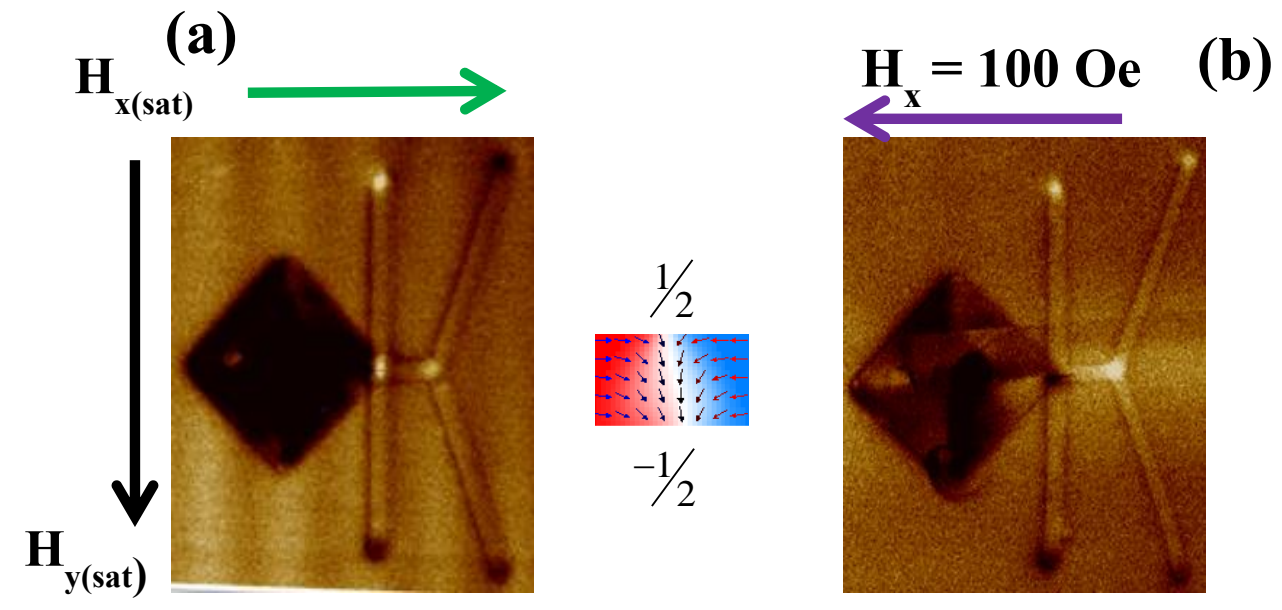

Fig. 3.9 Initial (a) and final (b) magnetization of the nanostructure when $-1 / 2 \sim+1 / 2 D W$ is injected. For this case, chirality selector is magnetized by applying a large transverse field $\left(-H_{y}\right)$ while the nucleation pad and the nanowire are saturated by applying a longitudinal field $\left(+H_{x}\right)$.

When a field strength of 100 Oe was applied along $-x$ direction, the magnetization direction of the upper branch of the detector was found to be switched as shown in 3.9 (b). 
This implies that a TTD with $+1 / 2 \sim-1 / 2$ edge defects was injected and moved into the upper branch of the Y-shaped detector.

In essence, regardless of the type of TDW, a DW with $+1 / 2 \sim-1 / 2$ edge defects always move into the upper branch of the Y-shaped detector while a DW with $-1 / 2 \sim+1 / 2$ edge defects into the lower branch of the Y-shaped detector as evident from the MFM imaging.

\subsubsection{Topological rectifier}

Fig. 3.10 shows the SEM image of a rectifier structure where an anyled rectangle was patterned onto the nanowire conduit. The rectifier has a width of $\sim 240 \mathrm{~nm}(\sim 2 \times w)$ and length of $\sim 480 \mathrm{~nm}(\sim 4 \times w)$, makes an angle of $\sim+11^{\circ}$ with respect to the horizontal axis.

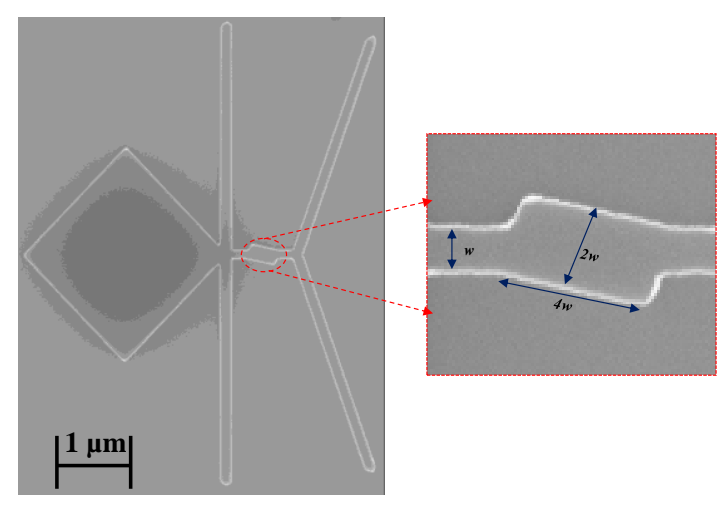

Fig. 3.10 SEM image of the rectifier structure. Inset shows the geometrical modulation along the nanowire. The rectifier structure has a length of $480 \mathrm{~nm}$ and width of $240 \mathrm{~nm}$. The nanowire has a width of $120 \mathrm{~nm}$ so as to stabilize transverse DW.

As shown in Fig. 3.11 (a), the initial configuration of the rectifier can be obtained by applying a transverse field of $\sim 500$ Oe along $+y$ direction and a longitudinal field of $\sim$ 500 Oe along $+x$ direction. The rectifier can be identified by a bright and dark contrast on opposite edges of the rectifier structure, owing to the accumulation of magnetic charges at the edge. When the magnetic field is applied along $-x$ direction, it leads to the injection of 
a TTU DW with edge defects $-1 / 2 \sim+1 / 2$ into the nanowire. The representative magnetization configuration of the device after the application of a field strength of 150 Oe along $-x$ direction can be seen in the MFM image of Fig. 3.11 (b).
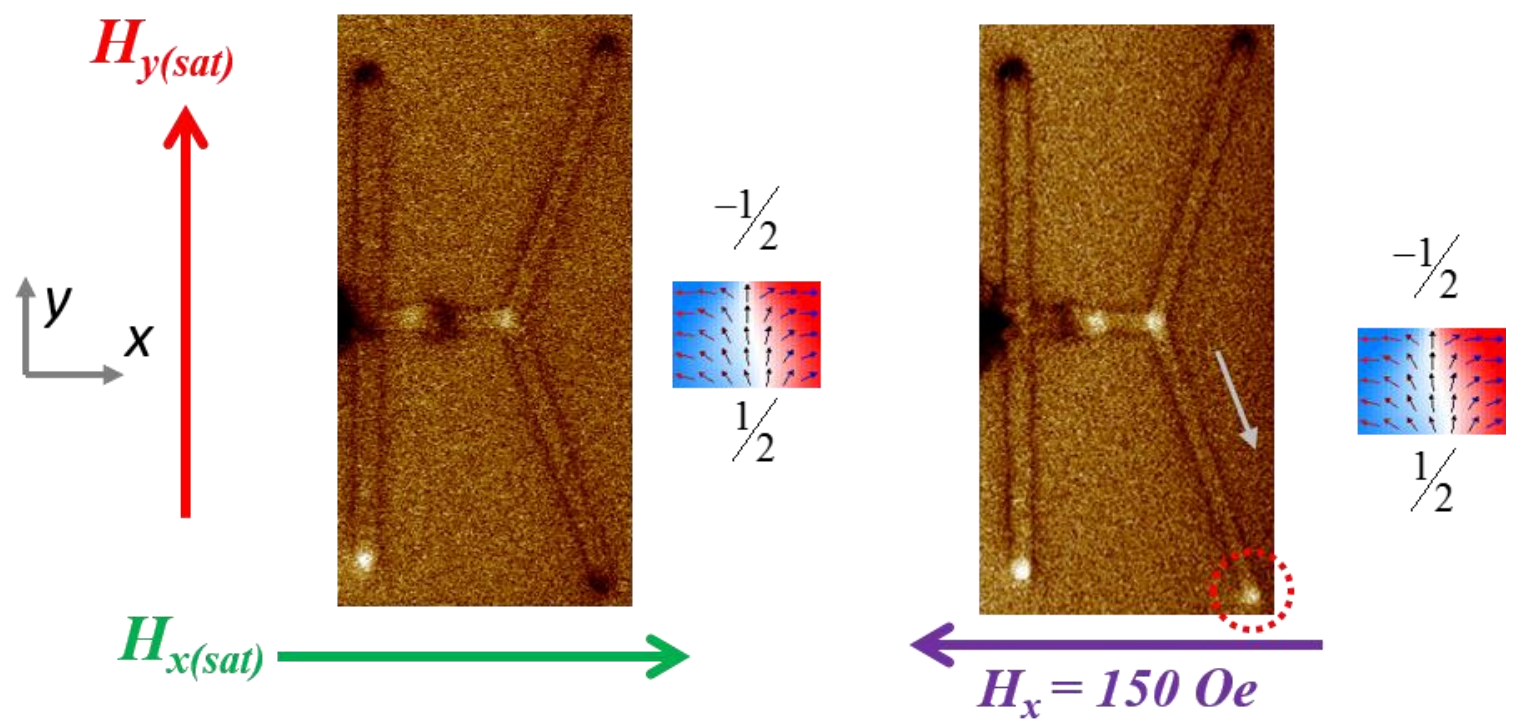

Fig. 3.11 MFM image of the rectifier: (a) after saturations along $+y$ and $+x$ directions respectively and (b) after applying a field of 150 Oe along -x direction.

This shows that the magnetization of the lower part of the detector is switched, implying that the output of the rectifier has $-1 / 2 \sim+1 / 2$ edge defects. As such, a TTU DW was not undergone any topological manipulation as it traverses through the structure.

Fig. 3.12 (a) shows the MFM image of magnetization configuration of the device when a chirality selector and nanowire are magnetized along the $-y$ and $+x$ direction respectively. As expected, the upper and lower edges of the chirality selector have bright and dark contrasts respectively. When a magnetic field strength of 150 Oe was applied along $-x$ direction, the lower branch of the detector switches to bright contrast as evident from the Fig. 3.12 (b). This represents that a TDW with edge defects $-1 / 2 \sim+1 / 2$, has reached 
the detector. As such, a rectifier always provides a TDW with edge defects $-1 / 2 \sim+1 / 2$ for any nucleated DW type such as $+1 / 2 \sim-1 / 2$ or $-1 / 2 \sim+1 / 2$.
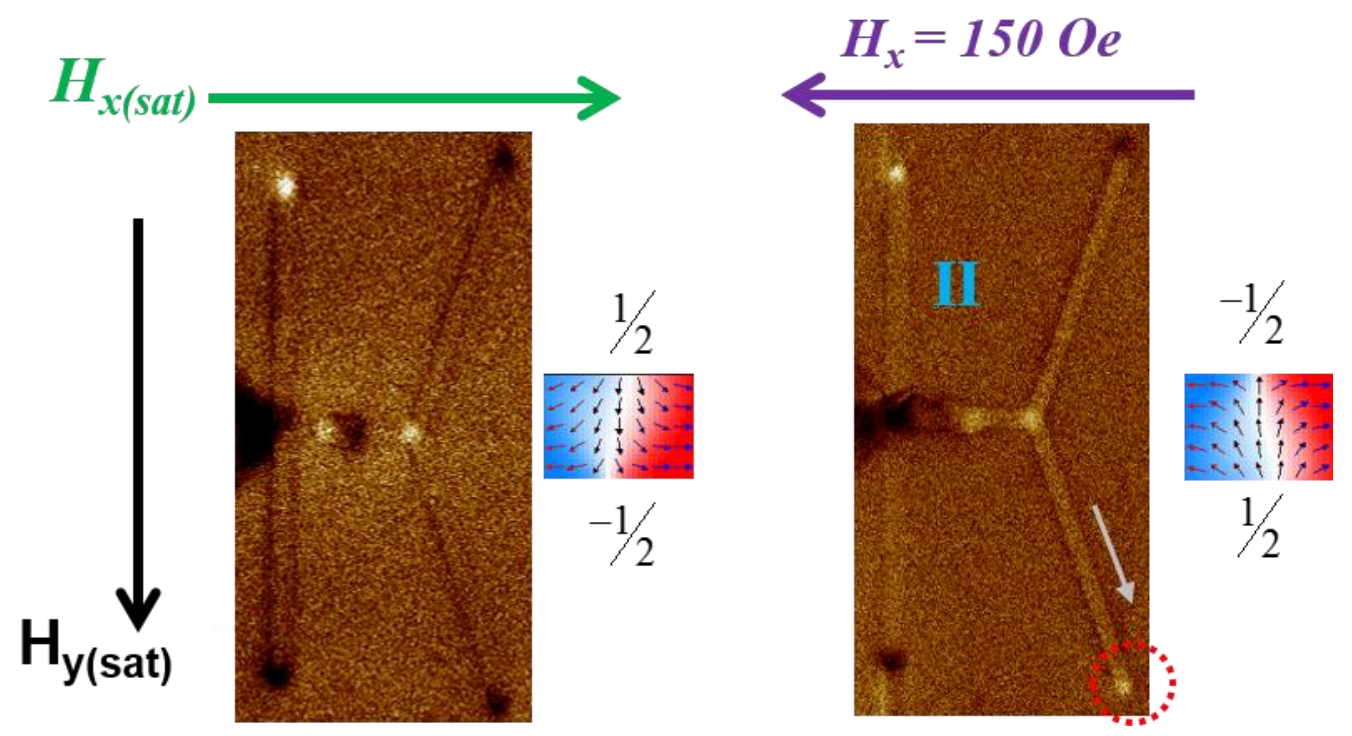

Fig. 3.12 MFM image of the rectifier: (a) when the chirality selector and nanowire conduit are magnetized along $-y$ and $+x$ directions respectively and $(b)$ after the application of $a$ field of 150 Oe along -x direction.

\subsubsection{Topological inverter}

To gain an insight on the detailed spin distribution with in an angled rectangle, micromagnetic simulation have been performed. The micromagnetic simulations reveal that $\alpha$ needs to be in the range of $3^{\circ}$ to $8^{\circ}$ for the structure to perform topological inversion of a TDW. This is due to the fact that the domain wall motion will be strongly influenced by the top right edge of the structure. Fig. 3.13 shows a schematic representation of the topological inversion of TDW. It can be clearly seen that the output of the topological inverter is always complement to the input TDW. 
Input

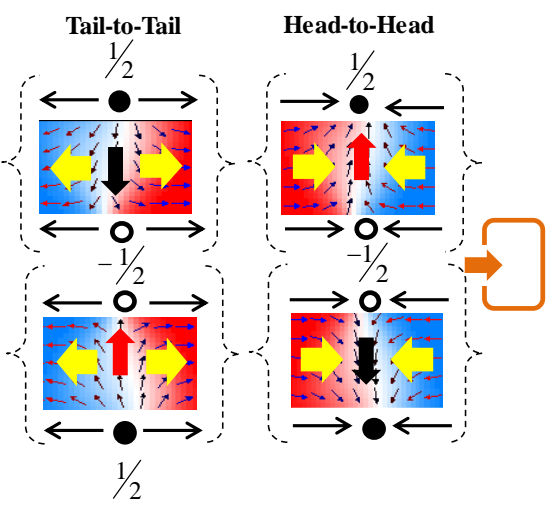

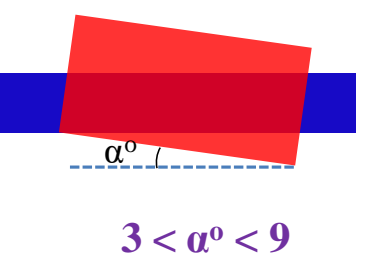

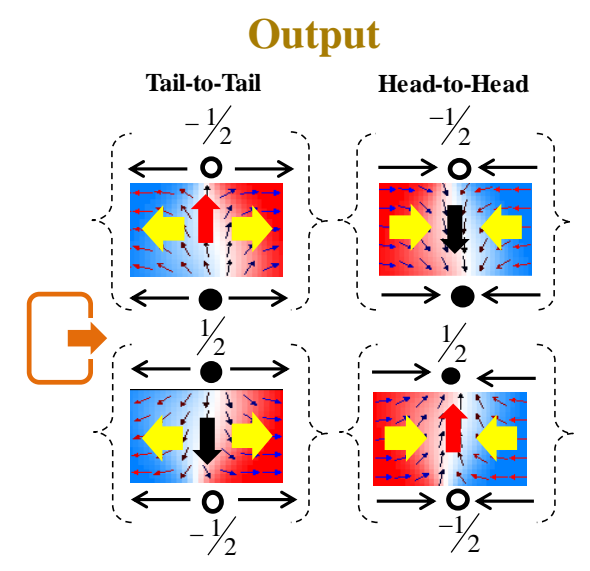

Fig. 3.13 Schematic representation of the topological inversion of a TDW. The angled rectangle makes an angle $3<\alpha^{\circ}<9$.

Micromagnetic simulations were performed by setting $\alpha$ to be $4^{\circ}$. As shown in Fig. 3.14 (a), a TTD DW $(+1 / 2 \sim-1 / 2)$ follows the same spin transformation as discussed for a rectifier. As shown in Fig. 3.14 (a), the inverter structure gives an output TTD with $-1 / 2$ $+1 / 2$ edge defects. However, when a TTU DW traverses through the inverter structure, it outputs a TTD DW. As described before, when the higher energy component $(+1 / 2)$ of the TTU DW encounters the lower edge of the conduit, where there is no potential barrier, the DW freely moves into the structure. Upon increasing the field strength, the $+1 / 2$ edge defect undergoes a transformation into a vortex core at the bottom right edge of the structure. As the transverse component of the domain wall is along $+y$-direction, the vortex core would adopt a clockwise configuration. The vortex core further moves along the vertical direction and then annihilated at the top right edge of the structure. As the spins on the right-hand side of the vortex core points "Down", a TTD DW with $+1 / 2 \sim-1 / 2$ edge defects exits the inverter structure. In essence, the structure performs the topological inversion. For instance, a HHU (HHD) DW traverses through the inverter structure and output the HHD (HHU) DW. 


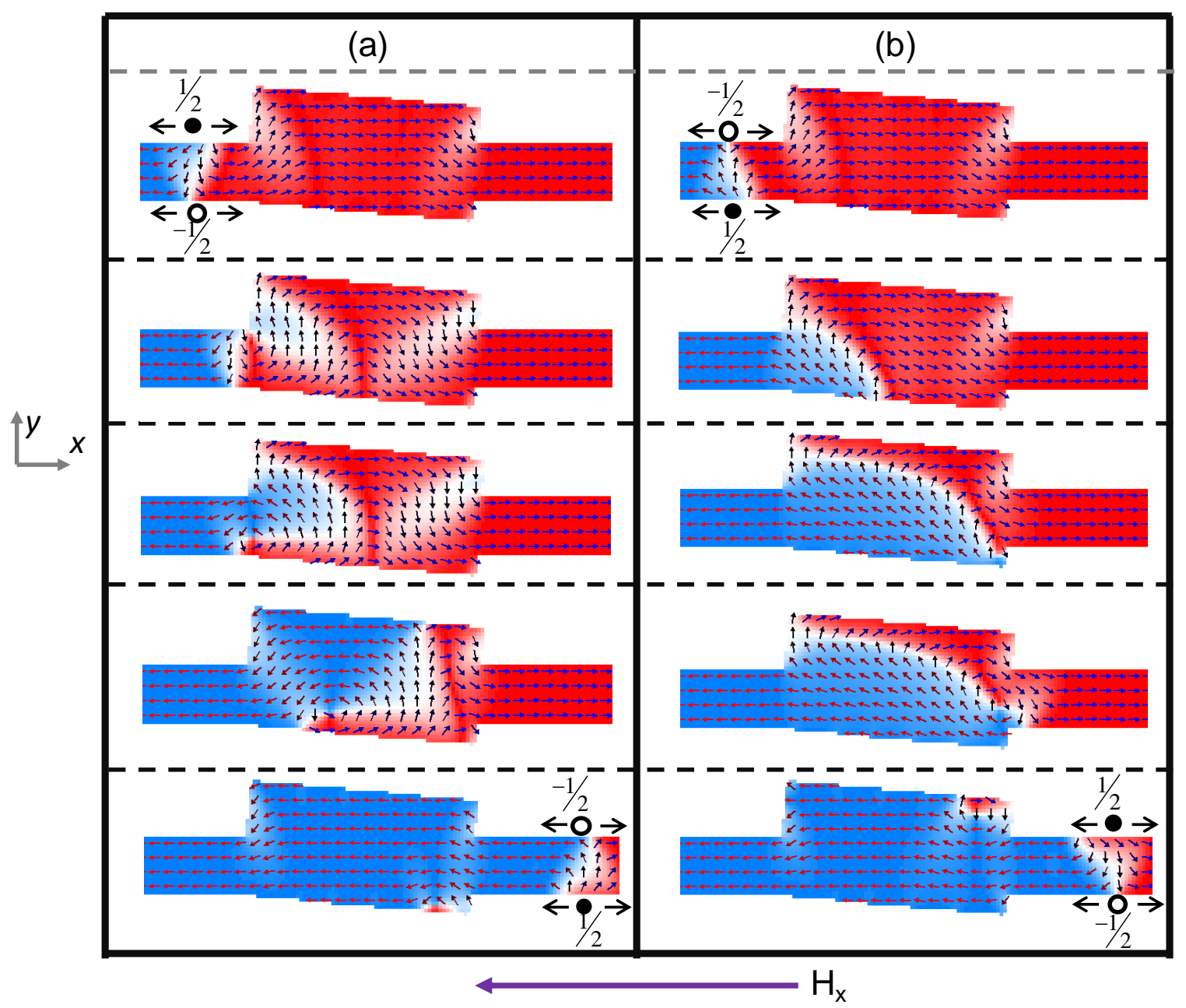

Fig. 3.14 Spin state evolution of as a TDW propagates through the inverter for (a) TT TDW with Down chirality $(+1 / 2 \sim-1 / 2)$ and (b) TT TDW with Up chirality $(-1 / 2 \sim+1 / 2)$.

\subsubsection{Experimental verification of inverter}

By changing the angle made by the rectangle structure with nanowire, topological inversion of the edge defects can be achieved. Fig. 3.15 shows the SEM image of an inverter, where the rectangle structure makes an angle of $\sim+4^{\circ}$ with respect to the nanowire long axis. 


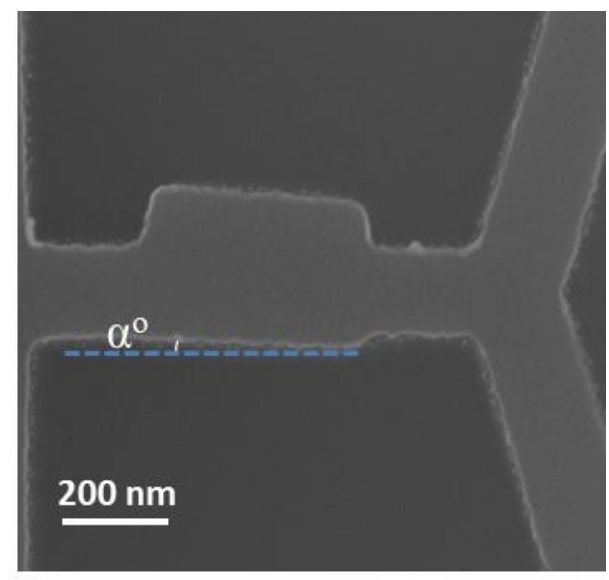

Fig. 3.15 SEM image of the inverter structure fabricated onto the nanowire conduit, where $\alpha=4 .^{\circ}$

Initially, the chirality selector is saturated along $+y$ direction and the nanowire conduit along $+x$ direction. Fig. 3.16 shows the representative magnetization configuration of the structure as obtained from MFM imaging. When a magnetic field is applied along $-x$ direction, a TTU DW is nucleated into the nanowire. When the magnetic field is further increased to 150 Oe along the same direction, resulting in the magnetization switching of the upper part of the Y-shaped detector. It indicates that the TTU DW transformed into a TTD DW via the topological inversion.

Fig 3.16(b)-I shows the magnetization configuration of the structure, when the chirality selector is saturated along $-y$ direction and the nanowire conduit along $+x$ direction. Upon increasing the magnetic field along $-x$ direction, now a TTD DW is injected into the nanowire. Following the application of a field of 150 Oe along the same direction, the magnetization configuration of the lower part of the detector is switched. It can be distinguished as a bright contrast at the lower part of the branch detector as shown in Fig. 3.16 (b)-II. As such, a TTD DW undergone a topological inversion, resulting in a TTU DW. 


\section{Tail-to-Tail}
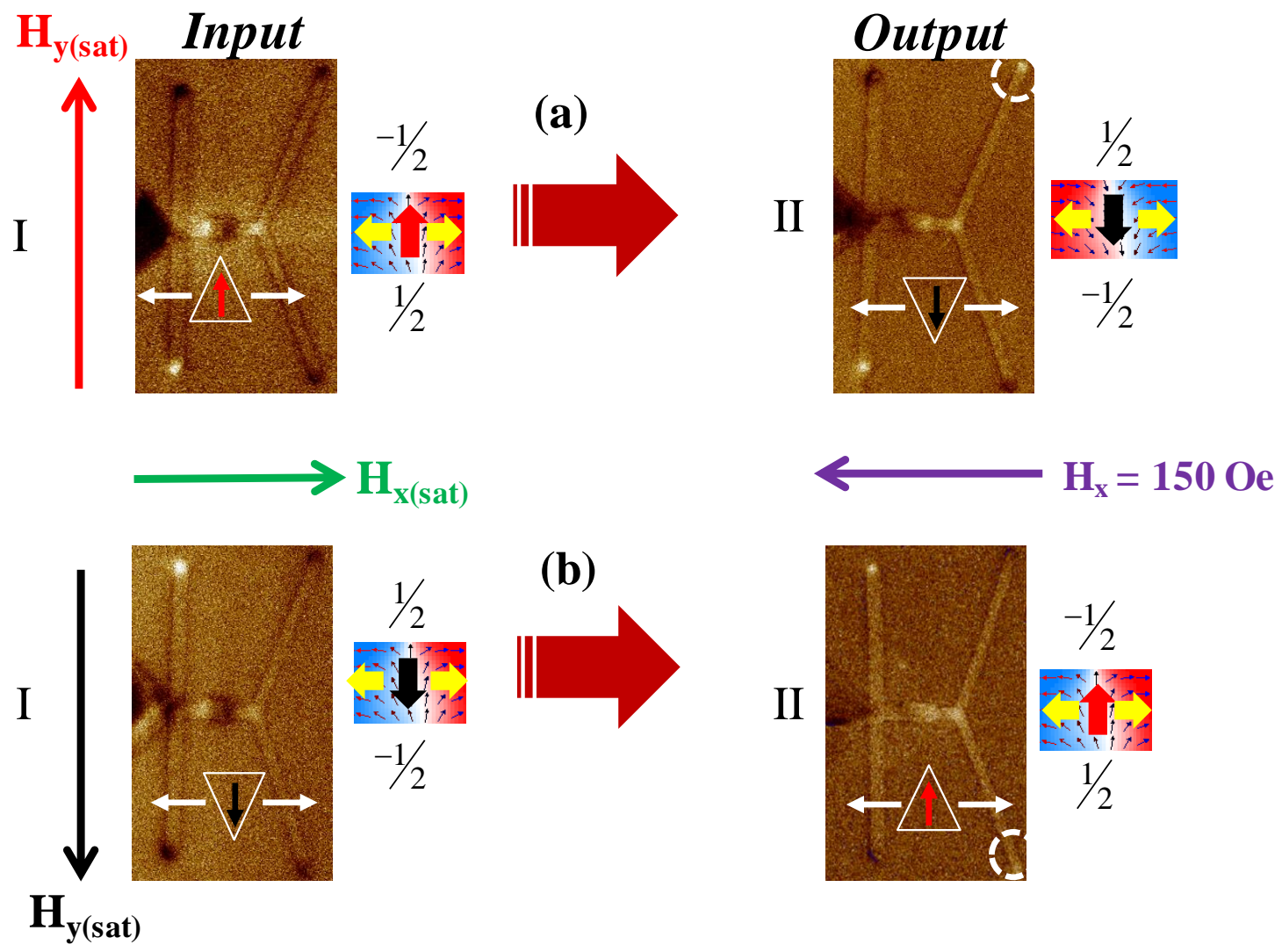

Fig. 3.16 MFM images of the topological inverter with initial and final configurations for; (a) TTU DW and (b) TTD DW.

Fig. 3.17 shows the inversion operation of a head-to-head (HH) DW. The initial magnetization configuration of the inverter when the chirality selector is magnetized along $+y$ and the nanowire along $-x$ is shown in Fig. 3.17 (a)-I. Upon increasing the magnetic field the opposite direction, i.e. $+x$ direction, a HHU TDW with $+1 / 2 \sim-1 / 2$ edge defects is injected into the nanowire conduit. Fig. 3.17 (a)-II shows the final magnetization configuration of the structure, where the lower part of the Y-shaped detector has switched. It is noted that a TDW with $+1 / 2 \sim-1 / 2$ edge defects is detected at the upper part of the $\mathrm{Y}$ - 
shaped detector while the TDW with $-1 / 2 \sim+1 / 2$ edge defects is detected at the lower part of the detector. As shown in Fig. 3.17 (a)-II, a TDW with $-1 / 2 \sim+1 / 2$ edge defects has moved to the lower part of the Y-shaped detector. This shows that the inverter has switched the topological defects of a HHU TDW $(+1 / 2 \sim-1 / 2)$ to a HHD TDW $(-1 / 2 \sim+1 / 2)$. It confirms the operation of the topological inversion of a TDW.

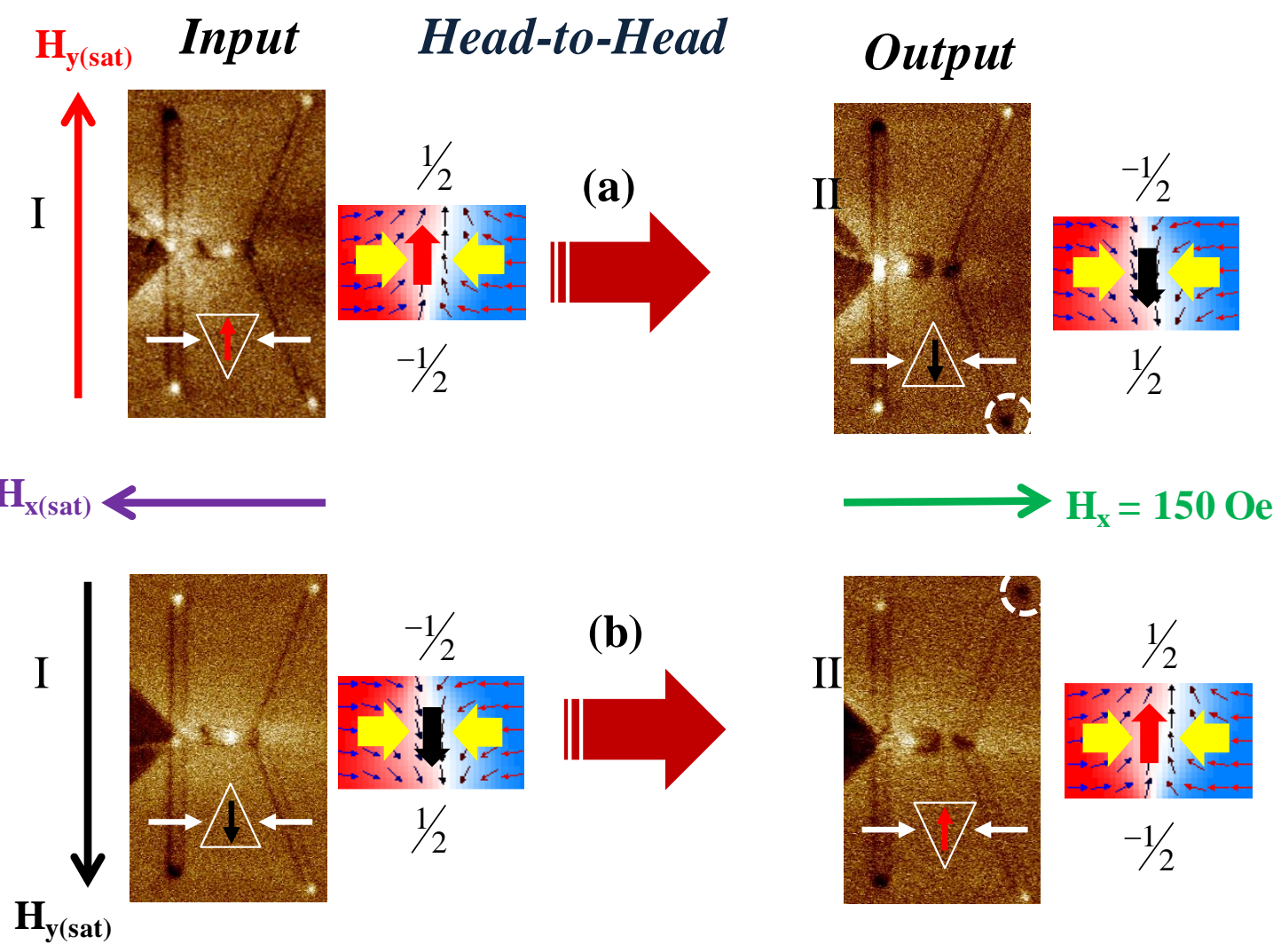

Fig. 3.17 MFM images of the topological inverter with initial and final configurations for; (a) HHU DW and (b) HHD DW.

Similarly, when a HHD TDW with $-1 / 2 \sim+1 / 2$ edge defects is nucleated in the nanowire conduit and is moved into the inverter structure, the output TDW from the inverter switches the upper part of the detector as shown in Fig. 3.17 (b)-II. This confirms that the operation of the inverter structure. 


\subsubsection{Reliability of the rectification/inversion operation}

In order to test the reliability of rectification and inversion operation based on DW profile, an array of the rectifier and inverter structures were fabricated and measured using MFM. For instance, Fig. 3.18 shows the MFM images for initial and final configurations for a $3 \times 3$ array of $-1 / 2 \sim+1 / 2$ rectifier structures. To nucleate a DW with specific chirality, the chirality selector (transverse nanowire) is first magnetized along the $+y$ direction, by applying a saturation field (500 Oe). The structure and nucleation pad are then saturated in $+x$ direction by applying a field of 500 Oe. Fig. 3.18 shows the initial configurations of the rectifier structures, where the transverse nanowire is characterized by a dark and bright contrast at the upper and lower edges respectively and the detector by dark contrasts at the lower and upper branches. By applying a linear field of 150 Oe along the $-\mathrm{x}$ direction, a DW is injected into the nanowire conduit and propagates through the structure. Due to the initial configuration of the rectifier structure, a Tail-to-Tail DW (TTDW) with "Up" chirality, with $-1 / 2 \sim+1 / 2$ topological charge, is injected in the nanowire. The TTDW switches the magnetization direction of lower branch of the detector as seen from the bright contrast in the final configuration in Fig 3.18. As the rectifier structure always provides an output of $+1 / 2 \sim-1 / 2$, the chirality of the input TTDW is flipped, to a TTDW with "Down" chirality. This in turn, will lead to the upper branch of the detector to switch. From the final configuration of the rectifier structure, it is noted that out of the nine structure, seven structures show the proper switching of the upper branch, as confirmed by the bright contrast from the MFM images. For sample $A_{3}$, the lower branch has switched, indicating that there was no rectification. 


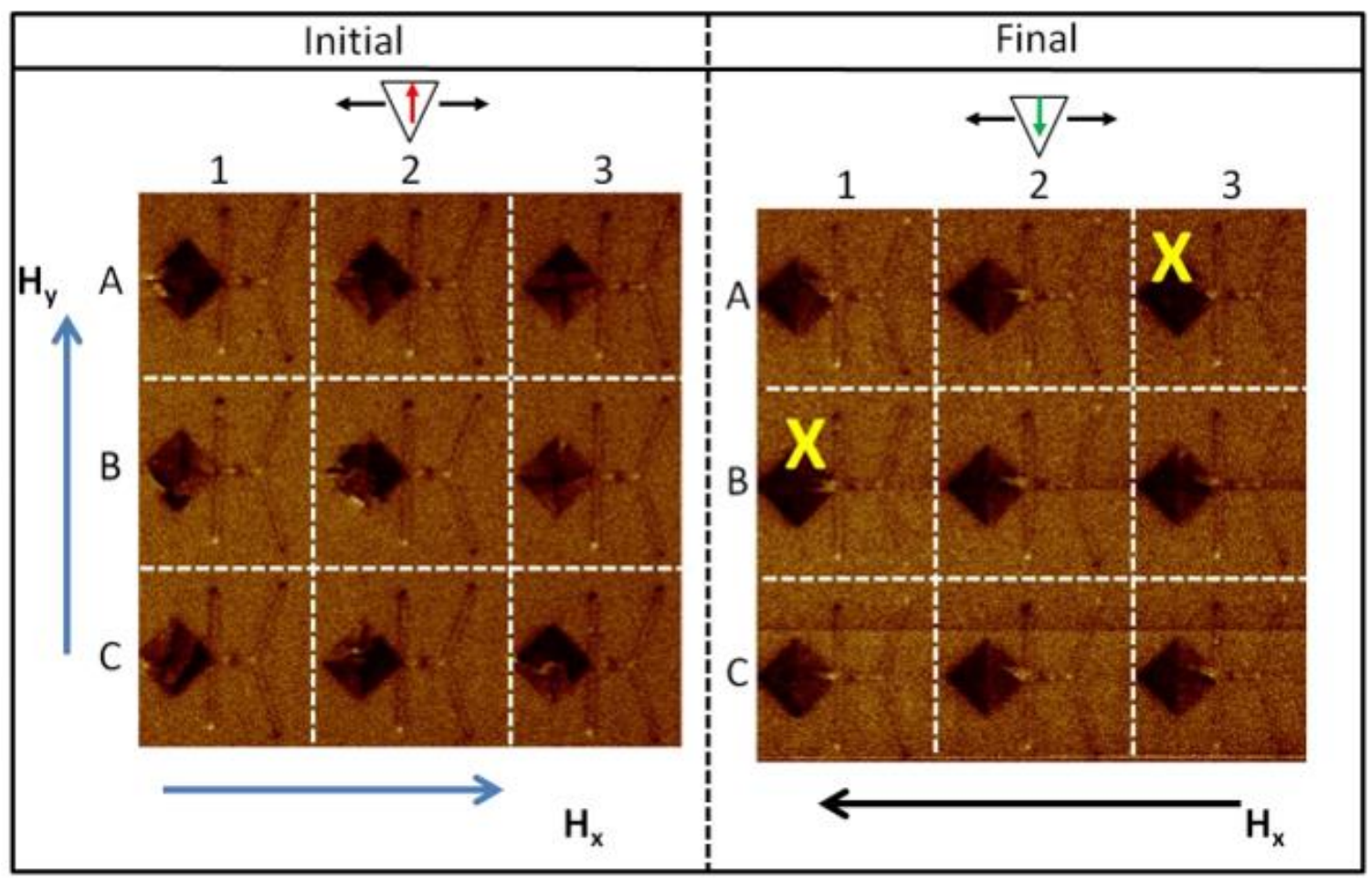

Fig. 3.18 MFM images for initial and final configurations of an array of rectifier structure. An in-plane field $(H x)$ applied along $x$-axis is used to saturate the nanostructure while field (Hy) applied along y-axis is used to set the chirality of a transverse DW.

Sample $\mathrm{B}_{1}$ on the other hand, does not exhibit a clear contrast at the detector. For devices that perform the logical operation properly, it has been observed that the subsequent results were repeatable. For these set of devices, the measurements were repeated for 20 times and probabilities for the successful rectification were plotted as shown in Fig. 3.19. In all the measurements, device $\mathrm{A}_{3}$ and $\mathrm{B}_{1}$ failed, whereas the remaining devices showed the expected configuration.

For devices that perform the logical operation properly, it was observed that for all measurements, they produced the same output. It implies that the DW rectification/inversion is reliable. 


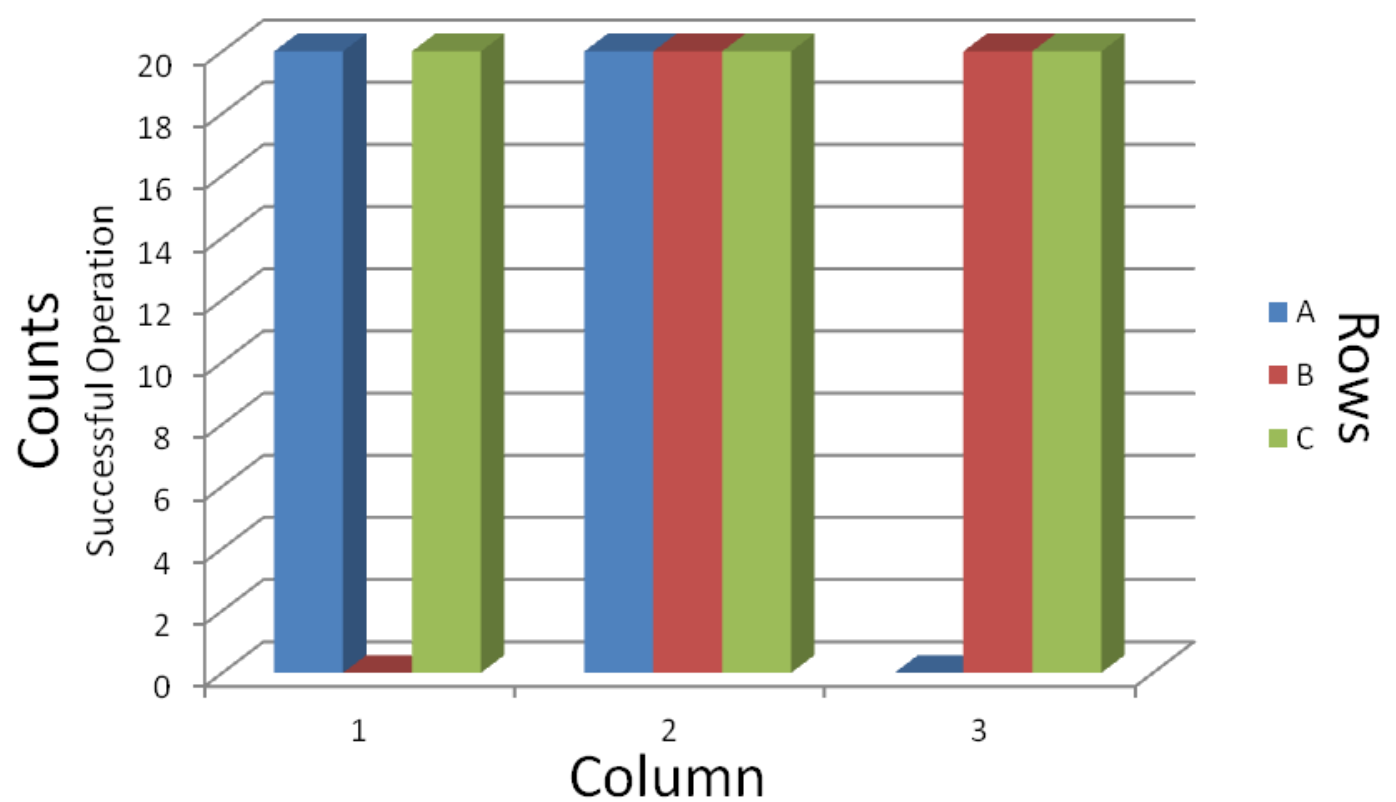

Fig. 3.19 Counts of successful rectification operation for 20 repeated measurements. The horizontal axis represents the column of devices and labels $A, B$ and $C$ represent the rows.

\subsection{Discussion}

In these experiments, the operations of logical NOT functionality as well as the rectification of a transverse DW based on its internal profile are demonstrated. The devices are clocked via linear magnetic field. Even though the fabricated devices have slight variations as observed by SEM, the topological rectification and inversion are still possible as evident from the MFM measurements. This implies that this technique is robust and can be applied for logic applications. The field required for the topological rectification/inversion operation is lower in experiments as compared to micromagnetic simulations and can be ascribed to thermally activated DW depinning [41]. A field of 150 Oe may lead to structural deformation, the walker breakdown and hence affects the integrity of the DW. The fidelity length of a DW decreases asymptotically to around $350 \mathrm{~nm}$ at higher 
fields [42]. Additionally, it has been reported that edge defects along the nanowire may also lead to shift walker breakdown to higher fields [43]. For the samples considered in this work, the length of the nanowire between nucleation pad and the detector is kept at $200 \mathrm{~nm}$. This is well below the reported fidelity length of the DW and ensures that the integrity of the input and output DWs are not compromised [26].

\subsection{Summary}

To summary, a novel approach to manipulate the topological defects of a TDW for logic applications was proposed. It employs controlled transformation of the TDW profile in a geometrically modulated nanowire. The relaxation process within the angles rectangle is found to be strongly influenced by an angle $\theta$, with which the rectangle orients with respect to the long axis of the nanowire. For $3<\theta<9$, the structure performs topological rectification. A topological rectifier gives a predetermined output which does not depend on the type of input DW. For $9<\theta<15$, the structure performs topological inversion, where the output is complimentary to the input. Slight change in the geometry of the structure does not influence the output, implying that this technique is robust and can be applied for various technologies. 


\section{References}

1. S. S. P. Parkin, M. Hayashi and L. Thomas, Science 320, 190 (2008).

2. D. A. Allwood, G. Xiong, C. C. Faulkner, D. Atkinson, D. Petit and R. P. Cowburn, Science 309, 1688 (2005).

3. M. T. Bryan, T. Schrefl and D. A. Allwood, Appl. Phys. Lett. 91, 142502 (2007).

4. D. A. Allwood, G. Xiong, M. D. Cooke, C.C. Faulkner, D. Atkinson, N. Vernier and R. P. Cowburn, Science 296, 5575 (2002).

5. M. Hayashi, L. Thomas, R. Moriya, C. Rettner and S. S. P. Parkin, Science 320, 209 (2008).

6. J. H. Franken, H. J. K. Swagten and B. Koopmans, Nat. Nanotech. 7, 499 (2012).

7. S. Glathe, M. Zeiseberger, U. Hebner and R. Mattheis. Phys. Rev. B81, 020412 (2010).

8. O. A. Tretiakov, D. Clarke, G.W. Chern, Y. B. Bazaliy, and O. Tchernyshyov. Phys. Rev. Lett.100, 127204 (2008).

9. T. Ono, H. Miyajima, K. Shigeto, K. Mibu, N. Hosoito and T. Shinjo, Science 284, 468 (1999).

10. G. S. D. Beach, C. Nistor, C. Knutson, M. Tsoi and J. L. Ersking, Nat. Mater.4, $741(2005)$.

11. X. Jiang, L. Thomas, R. Moriya, M. Hayashi, B. Bergman, C. Rettner and S. S. P. Parkin. Nat. Comm. 1, 25 (2010).

12. D. Atkinson, D. A. Allwood, G. Xiong, M. D. Cooke, C. C. Faulkner and R. P. Cowburn, Nat. Mater. 2, 85 (2003). 
13. L. K. Bogart, D. Atikson, K. O'shea, D. McGrouther and S. McVitie. Phys. Rev. B 79, 054414 (2009).

14. S. Lepadatu, A. Vanhaverbeke, D. Atkinson, R. Allenspach and C. H. Marrows, Phys. Rev. Lett. 102, 127203 (2009).

15. O. Tchernyshyov and G. W. Chern, Phys. Rev. Lett. 95, 197204 (2005).

16. A. Kunz, Appl. Phys. Lett.94, 132502 (2009).

17. L. Thomas, M. Hayashi, B. Moriya, C. Rettner, and S. S. P. Parkin. Nat. Comm. 3, 810 (2012).

18. I. Purnama, M. Chandra Sekhar, S. Goolaup, W. S. Lew, Appl. Phys. Lett. 99, 152501 (2011).

19. L. O'Brien, E.R. Lewis, A.F. Pacheco, D. Petit, R.P. Cowburn, J. Sampaio, and D.E. Read. Phys. Rev. Lett. 108, 187202 (2012).

20. L. O’Brien, D. Petit, H.T. Zeng, E.R. Lewis, J. Sampaio ,A.V. Jausovec, ,D.E. Read and R.P. Cowburn . Phys. Rev. Lett. 103, 077206 (2009).

21. A. Pushp, T. Phung, C. Rettner, B.P. Hughes, S.H. Yang, L. Thomas and S.S.P. Parkin. Nat. Phys.9, 505 (2013).

22. E. R. Lewis, D. Petit, L. O־Brien, A. Fernandez-Pacheco, J. Sampaio, A-V. Jausovec, H. T. Zeng, D. E. Read and R. P. Cowburn, Nature Mater. 9, 980 (2010).

23. E. R. Lewis, D. Petit, L. O־Brien, A.-V. Jausovec, H. T. Zeng, D. E. Read, and R.P. Cowburn, Appl. Phys. Lett. 98, 042502 (2011).

24. A. Kunz and S. C. Reiff, J. Appl. Phys. 103 07D903 (2008). 
25. M. T. Bryan, T. Schrefl, D. Atkinson, and D. A. Allwood, J. Appl. Phys. 103, 073906 (2008).

26. D. Burn and D. Atkinson, Appl. Phys. Lett.102, 242414 (2013).

27. Micromagnetic simulations were performed using the OOMMF code, available at http://math.nist.gov/oommf/. Accessed in June (2013).

28. D. Atkinson, D. S. Eastwood and L. K. Bogart, Appl. Phys. Lett. 92, 022510 (2008).

29. M. Chandra Sekhar, S. Goolaup, I. Purnama, and W. S. Lew, J. Appl. Phys. 115, 083913 (2014).

30. S. Goolaup, S. C. Low, M. Chandra Sekhar and W. S. Lew, J. Phys.: Conference Series 266, 012079 (2011).

31. D. Petit, A. V. Jausovec, D. Read and R. P. Cowburn, J. Appl. Phys. 103, 114307 (2008).

32. D.Petit, A. V. Jausovec, H. T. Zeng, E. Lewis, L.O’Brien, D. Read and R.P. Cowburn. Appl. Phys. Lett. 93, 163108 (2008).

33. G. Meier, M. Bolte, R. Eiselt, B. Kruger, D.H. Kim and P.Fischer, .Phys. Rev. Lett. 98, 187202 (2007).

34. A. Bisig, M. Stark, M.A. Mawass, C.Moutafis, J. Rhensius, J. Heidler, F. Buttner, M. Noske, M. Weigand, S. Eisebett, T. Tyliszczak, B. V. Waeyenberge, H. Stoll, G. Schutz and M. Klaui. Nat. Comm. 4, 2328 (2013).

35. E. R. Lewis, D. Petit, A.V. Jausovec, L. OBrien, D. E. Read, H. T. Zeng, and R. P. Cowburn, Phys. Rev. Lett. 102, 057209 (2009). 
36. C. Guite, I. S. Kerk, M. C. Shekar, M. Ramu, S. Goolaup and W. S. Lew. Sci. Rep.4, 7459 (2014).

37. K. Shigeto, T. Shinjo, and T. Ono, Appl. Phys. Lett. 75 2815, (1999)

38. N. Biziere, C. Gatel, R. L. Balier, M. C. Clochard, J. E. Wegrowe and E. Snoeck. Nano Lett. 13, 2053 (2013).

39. M. Hayashi, L. Thomas, C. Rettner, R. Moriya, X. Jiang and S. S. Parking, Phys. Rev. Lett. 97, 207205 (2006).

40. C. Murapaka, P. Sethi, S. Goolaup and W.S.Lew et al.Appl. Phys. Exp.7, 113003 (2014).

41. A. Himeno, S.Kasai and T.One.J. Magn . Magn. Mater. 286, 167 (2005).

42. E. R. Lewis, D. Petit, A.V. Jausovec, L. O’Brien, D. E. Read, H. T. Zeng, and R. P. Cowburn, Phys. Rev. Lett.102, 057209 (2009).

43. Nakatani, Y., Thiaville, A. \& Miltat, Nat. Mater. 2, 251 (2003). 


\section{Chapter 4}

\section{Edge defect mediated domain wall interactions}

\subsection{Introduction}

In this chapter, a novel method to generate single DW with $100 \%$ probability in the absence of both external magnetic field and long current pulses is presented. This method exploits the intrinsic edge stray field of the magnetic nanowire. The edge stray field influences the DWs which are generated by local Oersted field, resulting in the annihilation of a DW closer to the edge of the nanowire. Additionally, it was found that this method is faster than the conventional method of DW generation and requires very low current densities compared to the conventional method. Further, current induced DW driving was attempted and found that the critical current density required to drive the DW was reduced. Field induced DW interaction was investigated by using micromagnetic simulations from the stand point of device reliability. Interaction of two DWs with different topological charges lead to the formation of a bound state or mutual annihilation. The bound state forms a $360^{\circ} \mathrm{DW}$, while the mutual annihilation results in the generation of spin waves that are propagated along the nanowire. The amplitude of the spin wave mode was found to increase with the increase of field strength and it has the maximum amplitude along the middle of the nanowire width. The spin wave power drops exponentially as it propagates along the length of the nanowire. The decay coefficient of the spin wave power suggests that the power drops to $10 \%$ at a distance of $350 \mathrm{~nm}$ away from the annihilation point. It limits the minimum separation required for two DWs in a racetrack memory device in order to avoid data erroneous. 


\subsection{Motivation}

The DW motion in ferromagnetic nanowires has been proposed towards making non-volatile memory and logic devices [1-6]. The success of these devices relies on controlled DW injection, driving and detection. Intense research has been devoted to overcome the high current density requirement to move the DW [7-12]. The detection of the DW can be performed by using magnetic tunnel junction (MTJ), which are treated relatively well developed. The DW injection scheme is not fully established $[7,10,13,14]$. To date, DW generation via local Oersted field method is only proven to be the wellestablished method [14-17]. This method is very efficient and generate DWs in nanoseconds range. This method, however, suffers from random or stochastic DW generation. This method generates two DWs of opposite magnetic charges, a head-to-head $(\mathrm{HH})$ and a tail-to-tail (TT) DW, on each side of an injection strip line [18]. The interaction of these DWs lead to the formation of bound state or mutual annihilation that results in the stochastic DW generation $[13,18,19]$. The DW annihilation further leads to spin wave emission [20-21]. Micromagnetic simulations haves shown that the dynamics of a DW within a nanowire can be affected by the spin waves propagating at high speeds [22-23]. To overcome the stochastic DW generation, the minimum separation between two DWs should be $2.5 \mu \mathrm{m}[5,18]$. An external applied magnetic field or higher duration current pulses are used to push away the two DWs such that DW generation probability can be increased [1424]. Further, it was shown that the conventional method requires 100 pulses for reliable DW generation [24]. Applying an external magnetic field brings scalability issues and longer duration pulses produce lot of heat in the device. Therefore, developing a method that allows $100 \%$ DW generation probability is crucial for DW based memory devices. 


\subsection{Deterministic generation of single domain wall}

\subsubsection{Sample fabrication}

A multilayer stack of $\mathrm{Ta}(3 \mathrm{~nm}) / \mathrm{Ni}_{80} \mathrm{Fe}_{20}(10 \mathrm{~nm}) / \mathrm{Ta}(3 \mathrm{~nm})$ was deposited on $\mathrm{SiO}_{2}(300 \mathrm{~nm}) / \mathrm{Si}$ substrate using DC magnetron sputtering as shown in Fig. 4.1 (a). The bottom Ta layer is used to provide good adhesion and (lllllllll texture for the underlying $\mathrm{Ni}_{80} \mathrm{Fe}_{20}$ layer. The top Ta layer is used as a protective layer for underlying $\mathrm{Ni}_{80} \mathrm{Fe}_{20}$. Electron beam lithography and Ar ion milling were employed to pattern $300 \mathrm{~nm}$ wide nanowires. Both ends of the nanowire were tapered to avoid the nucleation of DWs from the edges of the nanowire. Fig. 4.1 (b) shows schematic of the patterned layers with nanowire at the centre. A second lithography process was carried out to pattern the DW nucleation line and electrical contacts to the NW. A large ground plane was also patterned around the device that helps to reduce electrical noise in the measurement and also serves as a common ground pad for high frequency RF probes.
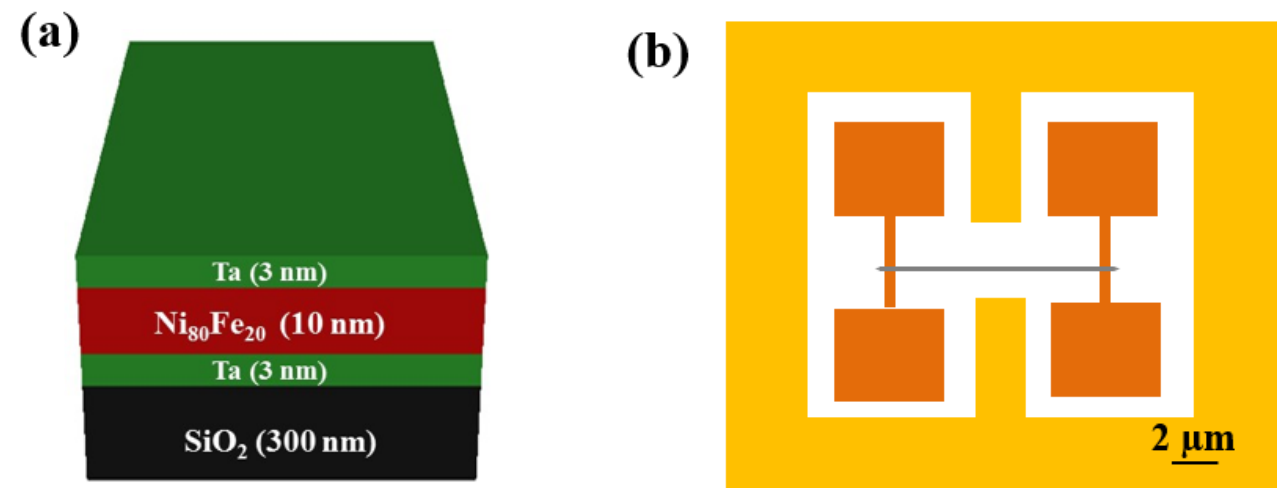

Fig. 4.1 (a) Schematic of the Ni80Fe 20 thin film stack structure. Bottom Ta layer acts as a seed layer while the top Ta layer protects from being oxidation. (b) Schematic of EBL design with different patterned layers. 
The high frequency RF probes are used to inject current pulses for DW generation and driving. An Ar reverse sputtering was used before the contacts deposition to remove any oxide layer. A complete device was obtained after the deposition of $\mathrm{Ta}(10 \mathrm{~nm}) / \mathrm{Cu}(80$ $\mathrm{nm}) / \mathrm{Au}(10 \mathrm{~nm})$ contacts followed by the lift-off.

\subsubsection{Measurement set up}

SEM image of a device with schematic of measurement setup laid on it is shown in Fig. 4.2. Electrodes $\mathrm{AA}^{\prime}$ and $\mathrm{BB}^{\prime}$ are used to inject and drive the $\mathrm{DW}$ in the nanowire respectively. The DW injection and driving measurements were performed on a commercial probe station equipped with a homemade electromagnet which generates an in-plane field of $\sim 1 \mathrm{kOe}$.

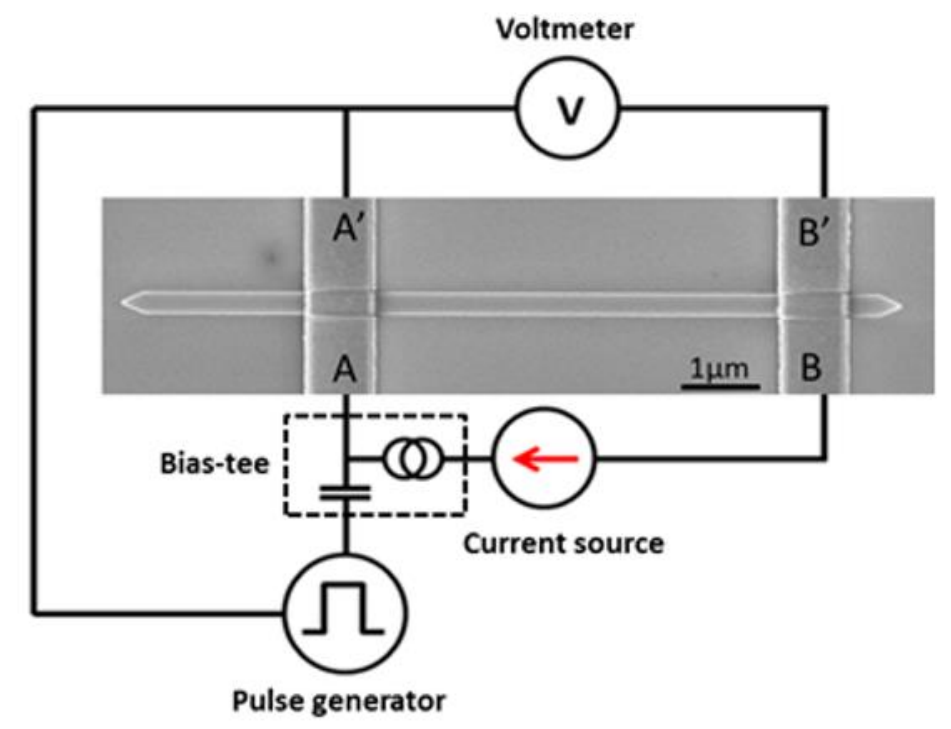

Fig. 4.2 SEM image of the device with schematic of the measurement setup. A nanowire has a width of $300 \mathrm{~nm}$ and two electrodes $A A^{\prime}$ ' and $B B^{\prime}$ ' are laid on top of the nanowire. A pulse generator is used to inject pulses for injection and driving. 
The RF probes which have a band width of $40 \mathrm{GHz}$ are used for DW injection and driving while the DC probes which have a band width of $5 \mathrm{GHz}$ are used to measure the resistance of nanowire. A pulse generator (Picosecond Pulse Labs 10300B) was employed to inject current pulses for DW injection and driving. A Bias tee which separates the DC source from the pulse generator was connected to the pulse generator. A DC current source (Keithley 2400) and a DC voltmeter (Keithley 2000) were employed to measure the anisotropic magneto resistance (AMR).

\subsubsection{Stochastic domain wall generation}

Due to negligible crystalline anisotropy $(k=0)$ of the permalloy $\left(\mathrm{Ni}_{80} \mathrm{Fe}_{20}\right)$, the magnetization is always constrained to follow the shape of the nanostructure. As a result, the magnetization of the $\mathrm{Ni}_{80} \mathrm{Fe}_{20}$ nanowire tends to align along the long axis. A DW can be injected by switching the magnetization locally. From the Maxwell's equation, a magnetic field can be generated when a current pass through a conducting wire. Fig. 4.2 shows the SEM image of a nanowire together with a conventional injection strip line where the injection strip line is placed $2 \mu \mathrm{m}$ distant from left extreme of the nanowire.

For DW generation, the nanowire was initially saturated with $\sim 1$ kOe magnetic field applied along its long axis. When a current density $(\mathrm{J})=2.6 \times 10^{12} \mathrm{~A} / \mathrm{m}^{2}$ was injected through a strip line from A to A', two DWs were generated underneath it. The potential difference between electrodes AA' and BB' was measured directly using DC probes and a DC current of $100 \mu \mathrm{A}$ was passed simultaneously. The DC bias current is significantly lower than the driving current density and does not induce any DW motion. Due to AMR effect, the presence of the DW can be directly evident from the drop in resistance. When the resistance drop was sustained for longer than $60 \mathrm{~s}$, the DW generation was treated 
successful. Further, the generated DWs can be annihilated by reversing the polarity of current pulse, injecting from electrode $\mathrm{A}^{\prime}$ to A. Fig. 4.3 shows the DW generation probability attempted in 50 times for various pulse durations. The maximum probability that can be attained by using this method is less than $60 \%$ and it was obtained for a pulse duration of 50 ns. Fig. 4.3 (b) shows the sequence of resistance changes across the nanowire as a function of time, implying multiple DW injections and mutual annihilations.
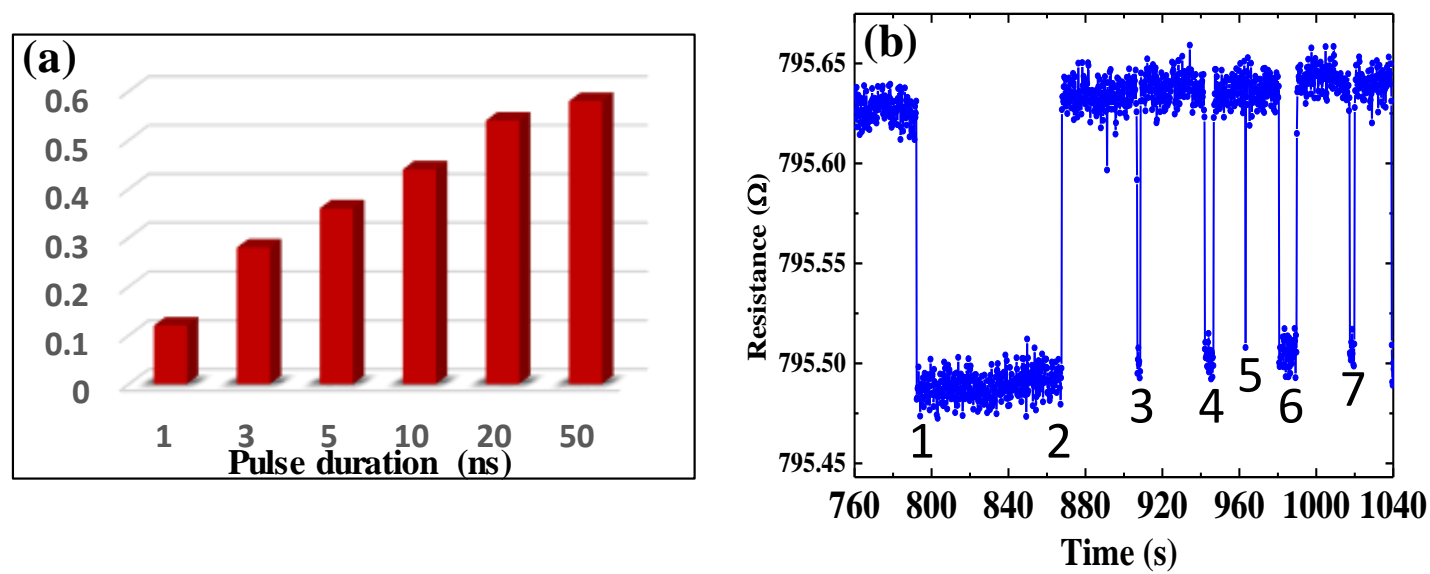

Fig.4.3 (a) Probability for the DW generation at a fixed current density of $2.1 \times 10^{12} \mathrm{Am}^{-2}$ (b) Measured AMR signal corresponds to a combination of multiple successful and failed.

The initial resistance of the nanowire was $795.625 \pm 0.025 \Omega$. After injecting a current pulse at $\mathrm{t}=30 \mathrm{~s}$, the resistance was found to be dropped to $795.485 \pm 0.025 \mathrm{~V}$, as represented by marker 1 . This change in resistance was $0.14 \pm 0.025 \Omega$. The change in resistance of $0.14 \Omega$ corresponds to a transverse DW [5]. The DW generation is treated to be successful as the resistance is sustained for longer than $60 \mathrm{~s}$. When a reverse current was applied at $\mathrm{t}=110 \mathrm{~s}$, the resistance of the nanowire switches back to the initial resistance, as indicated by marker 2 in Fig. 4.3 (b). Further attempts of injection with the same current density did not provide any stable resistance change. The markers $3,4,5,6,7$ show the 
resistance drop, but they are stable only for a limited period of time. The DW generation was thus considered to be fail and it is possibly owing to the mutual annihilation of the nucleated DWs.

As shown in Fig. 4.4, the direct observation of the DW generation was performed by using magnetic force microscope (MFM). As can be seen, two coupled DWs are generated beneath the injection strip line. DW driving was attempted in the absence of an external magnetic field with a current density of $1.3 \times 10^{12} \mathrm{Am}^{-2}$. But no appreciable change in the resistance of the nanowire was observed. It can be due to a very small portion of the current interacts with the DWs and large portion of current was shunted by the strip line.

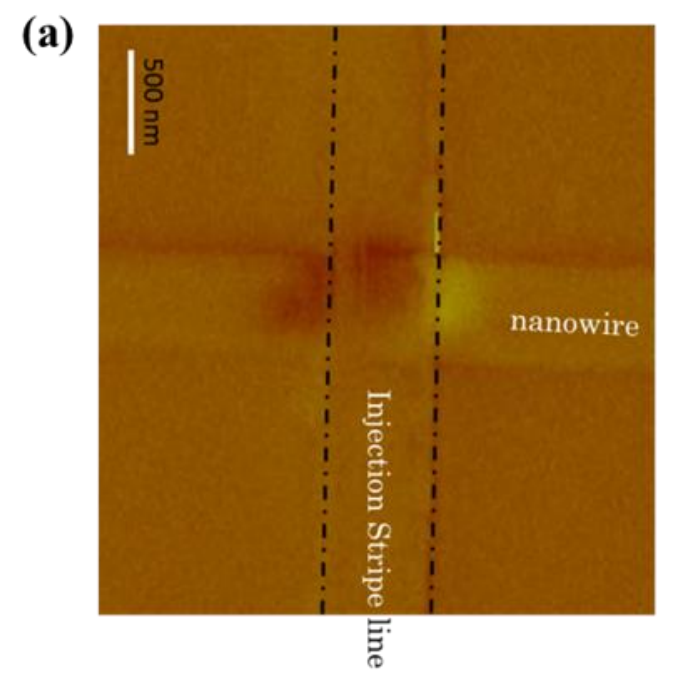

(b)

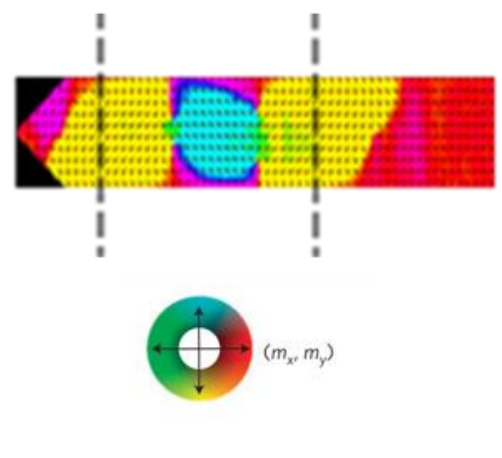

Fig. 4.4 (a) An MFM image of a NW comprises of two DWs generated underneath the injection strip line. (b) An equivalent micromagnetically simulated DW generation, where two DWs can be underneath the injection strip line

The conventional injection scheme where no external field is applied suffers from few issues. Firstly, the DW generation probability was found to be less than $100 \%$, implying successful DW generation needs more than one current pulse. Secondly, when the 
DWs are generated successfully, they remain underneath the strip line. It makes DW driving difficult as only a fraction of the current density will interact with the DW.

\subsubsection{Single domain wall generation}

To overcome these issues, a novel DW injection method was proposed where the stray field from the edge was exploited for the single DW generation. Fig. 4.5. shows the SEM image of the device where a strip line of $800 \mathrm{~nm}$ wide was positioned at a distance of $450 \mathrm{~nm}$ distant from the left extreme of the nanowire. Initially, the nanowire was saturated with an in-plane field of $1 \mathrm{kOe}$ along the nanowire long axis. A current density of $J=$ $1.1 \times 10^{12} \mathrm{Am}^{-2}$ with a pulse duration of $5 \mathrm{~ns}$ was subsequently injected into strip line from $A$ to $A^{\prime}$.

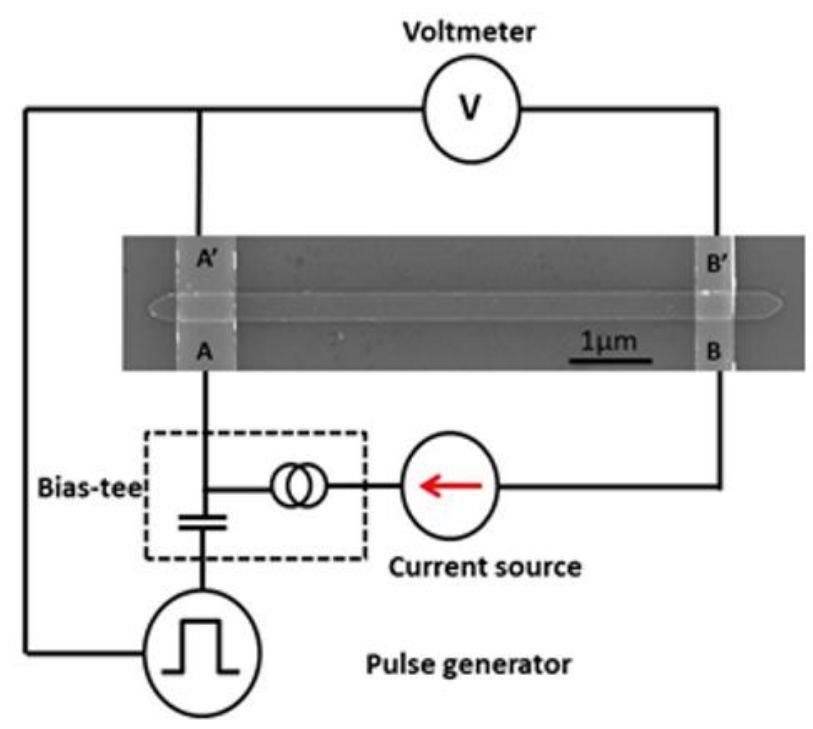

Fig. 4.5 SEM image of the proposed device structure with the measurement setup.

The DW generation was observed by in situ AMR measurement where the magnitude of the drop in resistance was found to be comparable to the previous method. 
Direct observation of the single DW generated was obtained by MFM imaging. As shown in Fig. 4.6 (a), the DW is moved few nanometers away from the injection strip line AA'. This confirms the single DW generation. Whereas, the DW closer to the left edge of nanowire was annihilated. The single DW generation probability was studied for different current densities ranging from $6.9 \times 10^{11} \mathrm{Am}^{-2}$ to $1.1 \times 10^{12} \mathrm{Am}^{-2}$. The pulse duration was varied from 1 to 100 ns. Fig. 4.6 (b) shows the probability of DW generation as a function of pulse duration for different current densities. The probabilities were estimated for total attempts of 50. Like two DWs generation method, the same condition was imposed for successful single DW generation method. $100 \%$ probability for the single DW generation was observed for two different current densities of $1.0 \times 10^{12} \mathrm{Am}^{-2}$ and $1.1 \times 10^{12} \mathrm{Am}^{-2}$ when the pulse duration exceeds $20 \mathrm{~ns}$ and $10 \mathrm{~ns}$ respectively.

(a)
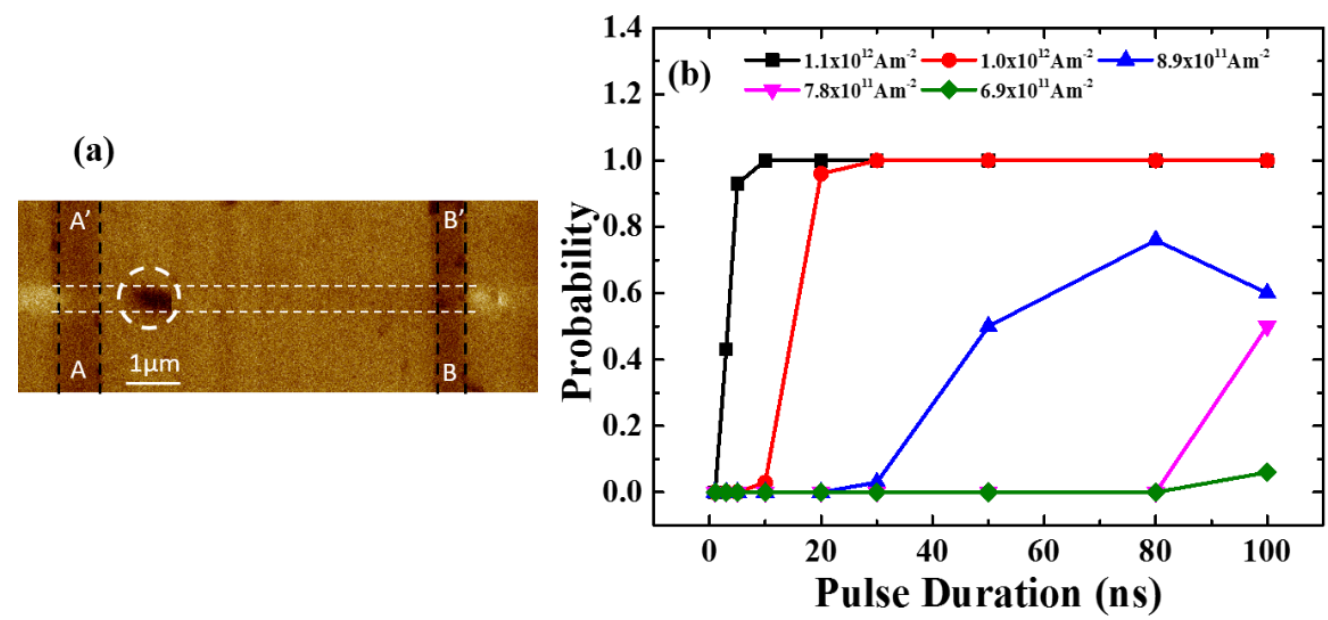

Fig. 4.6 (a) MFM image of the single DW generation. (b) DW injection probability as a function of pulse duration for different injection current densities. 


\subsubsection{Current induced single domain wall motion}

After successfully injecting a single DW using edge field assisted method, DW driving using current pulses was attempted. A current density of $1.3 \times 10^{12} \mathrm{Am}^{-2}$ with a pulse duration of $10 \mathrm{~ns}$ for two pulses was injected to drive the single DW which was generated in the previous experiment as shown in Fig. 4.6 (a). The amplitude and pulse duration of the current pulse can be varied such that the position of the DW can be controlled between AA' to BB'. The voltage was measured after each pulse to ensure that the DW was driven out of the section AA'-BB'. A DC bias current of $100 \mu \mathrm{A}$ was simultaneously passed through the nanowire from BB' to AA', As shown in Fig. 4.7 (a), MFM image confirms that the DW was displaced $\sim 2 \mu \mathrm{m}$ away from its initial position. A second DW was generated by injecting current pulse with a current density, $J=1.1 \times 10^{12} \mathrm{Am}^{-2}$ and pulse duration of $5 \mathrm{~ns}$ from $A$ ' to $A$. The second DW generation is evident from the MFM imaging as shown in Fig. 4.7 (b). The MFM image shows bright and dark contrasts for the two DWs, indicating the presence of opposite magnetic charges. A transverse DW is characterized by a magnetic charge.

(a)

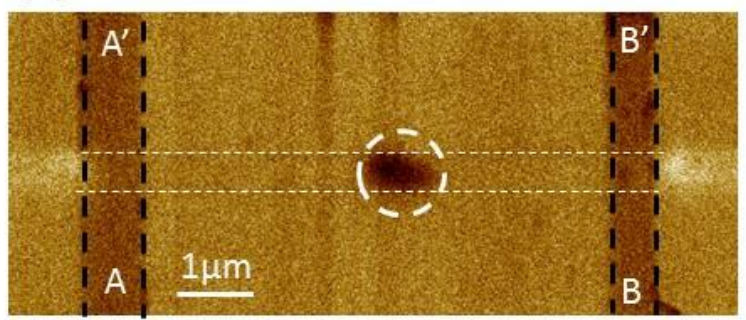

(b)

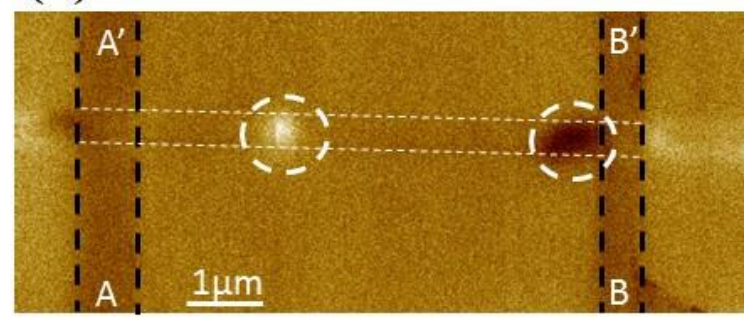

Fig. 4.7 (a) MFM image of the current induced domain wall motion after deterministic generation of single DW injection. (b) MFM image of two deterministically-generated DWs. 
A HH DW, where the two domains converge at the DW, possesses positive charge while a TTDW, where the two domains diverge at the DW, possesses negative charge. The dark and bright contrasts for the two DWs are due to attractive and repulsive interactions between the tip and local magnetization of the DW.

It has been shown that $100 \%$ DW generation probability can be attained by using edge-field-assisted method. Unlike two DWs generation method, this method does not allow the mutual annihilation of the DWs as evident from the in-situ AMR measurement. For failed attempts of DW generation, the resistance of the nanowire was not changed. This implies that the DW generation was successful every time and a single DW was nucleated every time. This eliminates the possibility of mutual annihilation of the generated DWs. $100 \%$ DW generation probability can be attained beyond a critical value of current density and pulse duration. Additionally, the single DW is always pushed away from the injection strip line. As such, this makes the DW driving easy because the driving current that passes through the nanowire interacts with the DW.

\subsubsection{Micromagnetic Simulation}

To substantiate the observed results, micromagnetic simulations have been performed by using LLG 3D micromagnetic simulator [25]. The standard material parameters of $\mathrm{Ni}_{80} \mathrm{Fe}_{20}$ were used in our simulations: Saturation magnetization $M_{s}=8.6 \times$

$10^{5} \mathrm{~A} / \mathrm{m}$, magnetocrystalline anisotropy $k=0$ and exchange stiffness constant $\left(A_{e x}\right)=1.3 \times$ $10^{-11} \mathrm{~J} / \mathrm{m}$. The damping constant $(\alpha)$ and non-adiabatic spin-torque constant $(\beta)$ were assumed to be 0.01 and 0.04 respectively. $1 \mu \mathrm{m}$ wide strip line was overlaid orthogonally onto $300 \mathrm{~nm}$ wide and $10 \mathrm{~nm}$ thick nanowire. Fig. 4.8 shows the spin configuration of the 
DW nucleation for different distances, $d$ between the injection strip line and left extreme of the nanowire and different pulse durations.

Two DWs were initially generated below the injection strip line. A pulse current density $(\mathrm{J})$ of $1 \times 10^{12} \mathrm{Am}^{-2}$ with a pulse duration of $1 \mathrm{~ns}$ was then injected through the strip line. The rise and fall times of the current pulse are fixed at $300 \mathrm{ps.}$

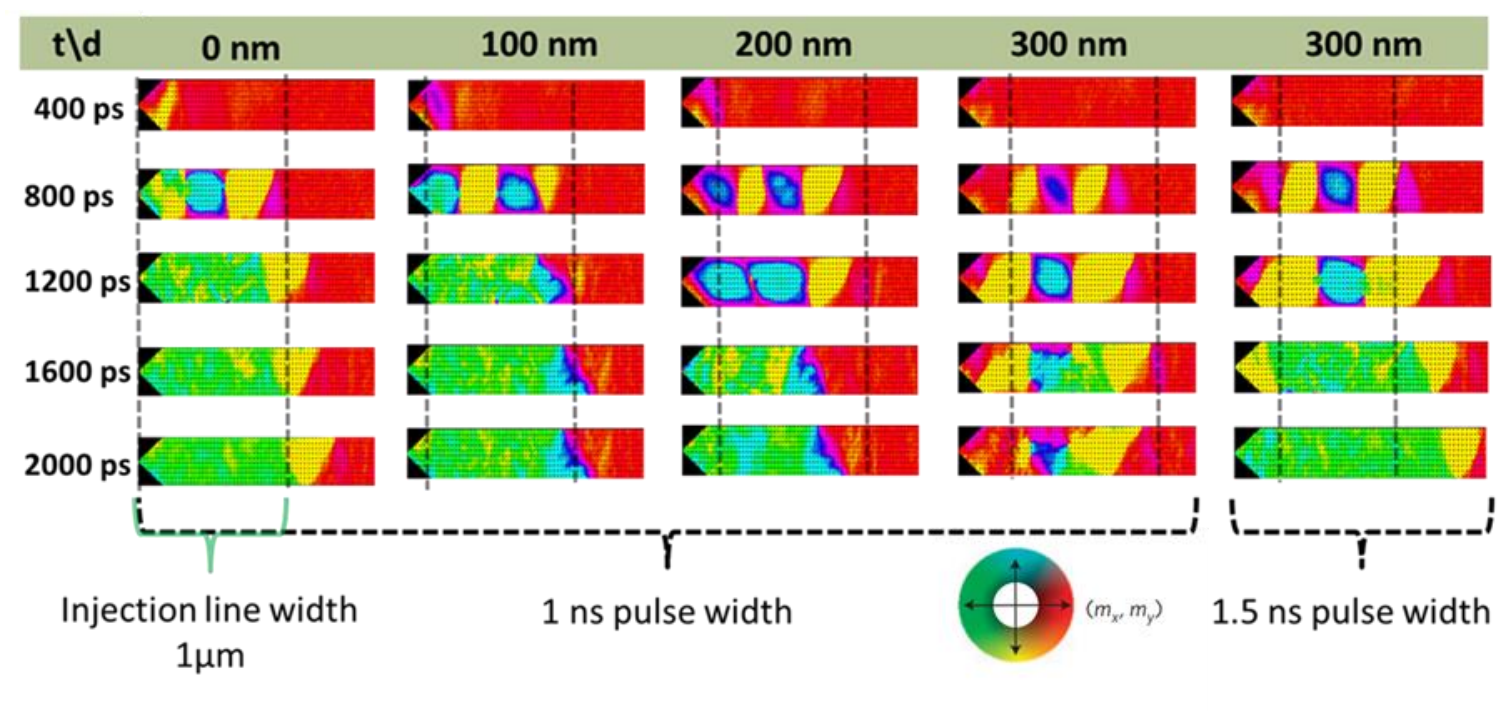

Fig. 4.8 Snapshot images of micromagnetic simulations during the temporal evolution of the DW generation for different separations of the NW edge to the strip line

When the injection strip line was aligned along the edge of the nanowire, for $d=0$ $\mathrm{nm}$, single DW was remained after the current pulse injection. This DW was pushed away from the strip line and moved towards the center of the nanowire. The other DW was pushed towards the edge of the nanowire and mutually annihilated upon interacting with the stray field. In contrast, as the spacing between the injection strip line and the edge of the NW was varied, i.e. for $\mathrm{d}=100$ and $200 \mathrm{~nm}$, the stabilization of a single DW takes more time and DWs generate underneath the strip line. The increase in the separation between the edge 
and strip line reduces the effect of edge stray field on the DW generation, causing the mutual interaction between the two DWs become more pronounced. It results in the more complex behavior of the reversal process below the injection strip line. For $\mathrm{d}=300 \mathrm{~nm}$ with $1 \mathrm{~ns}$ pulse duration, no well-defined DW formation is observed. However, when the duration of the pulse was raised to $1.5 \mathrm{~ns}$, successful DW generation was observed. For longer pulse duration, the Oersted field from the strip line sustains for longer periods of time, pushing the DW closer to the edge. Further, the influence of the edge stray field on the DW becomes more significant. As a result, the mutual annihilation between the edge stray field and DW takes place. This confirms the single DW generation. Fig. 4.9 (a) shows the plot of critical current density $J_{c}$ for the single DW generation as a function of distance, $d$. The pulse duration was kept constant at $1 \mathrm{~ns}$.
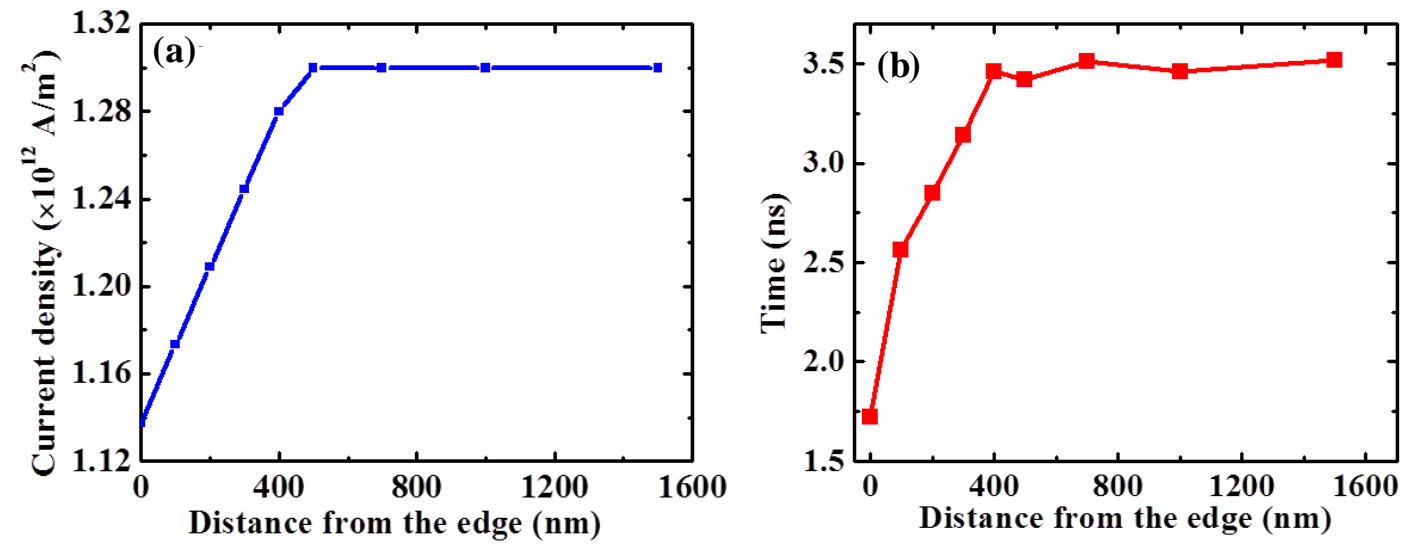

Fig. 4.9 (a) Plot of critical current density for the successful generation as a function of distance, $d$ for a fixed pulse duration. (b)Plot of stable domain wall formation time as a function of distance, $d$ for a fixed current density and pulse duration.

As shown in the Fig. 4.9 (a), $J_{c}$ increases linearly up to $d=500 \mathrm{~nm}$. For $d>500$ $\mathrm{nm}$, the required critical current density to stabilize the single DW remains constant. The 
increase in critical current density with d can be correlated to the decrease in the strength of the edge stray field. Fig. 4.9 (b) shows the plot of time needed to generate single DW for different spacing, $d$. It can be seen that the plot follows the same trend as that of the spacing, d. For larger separation, the time needed for the DW evolution would be longer. For $\mathrm{d}>$ $500 \mathrm{~nm}$, the time required to form a stable single DW remains constant. As shown in Fig. $4.9(a, b)$, the saturation in nucleation time and critical current density indicates that the effect of stray field on the DW generation is significant only up to $d=500 \mathrm{~nm}$. Further, the nucleation time for $d=0 \mathrm{~nm}$ is smaller than that of $d=500 \mathrm{~nm}$, implying that the edge field assisted single DW generation is twice as fast as the conventional method. Additionally, the critical current density required for single DW generation when $d=0$ is also lower than the critical density required when $d>500 \mathrm{~nm}$. The micromagnetic simulations provide a qualitative analysis of the DW generation.

\subsection{Spin wave emission via domain wall annihilation}

In this section, a detailed investigation of spin wave emission due to transverse DW collision is presented by means of object oriented micromagnetic framework (OOMMF) software [26]. A rectangular nanowire with $30 \mu \mathrm{m}$ length, $100 \mathrm{~nm}$ width and 6 $\mathrm{nm}$ thickness was discretized into cell size of $5 \times 5 \times 6 \mathrm{~nm}^{3}$. The length of the nanowire was chosen so as to avoid spin wave reflections from the end points of the nanowire. The standard material parameters of permalloy $\left(\mathrm{Ni}_{80} \mathrm{Fe}_{20}\right)$ were considered in the simulations: exchange stiffness constant $A_{e x}=1.3 \times 10^{-11} \mathrm{~J} / \mathrm{m}$, saturation magnetization $M_{s}=8.0 \times 10^{5}$ $\mathrm{A} / \mathrm{m}$, damping constant $\alpha=0.01$ and magnetocrystalline anisotropy $K=0$. As shown in schematic of Fig. 4.10, firstly, a transverse head-to-head $(\mathrm{HH})$ and tail-to-tail (TT) DWs were nucleated at a distance of $-13 \mu \mathrm{m}$ and $+13 \mu \mathrm{m}$ away from the center of the nanowire 
respectively. The two DWs have the same chirality, with transverse components pointing along $-y$ axis. An external in-plane magnetic field, $H_{a}$ is then applied along $+x$ direction. Driven by the external field, the HH and TT DWs move towards the center of the spin wave, where they collide and annihilate each other. The annihilation is possible because the two DWs have opposite edge defects.

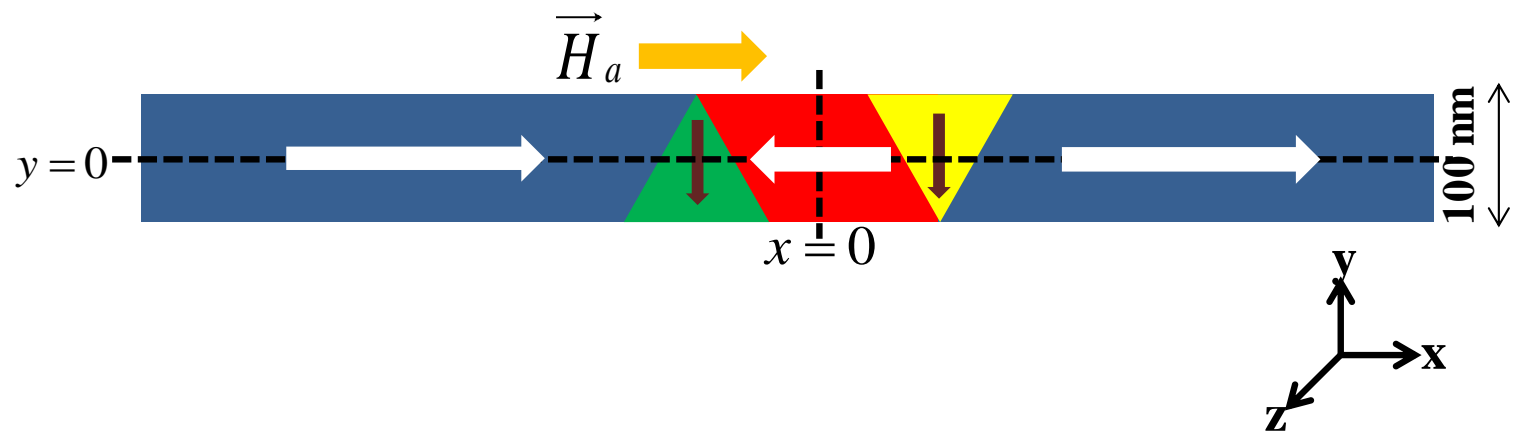

Fig. 4.10 Schematic representation of the model employed in our micromagnetic simulations: A head-to-head (green color) and a tail-to-tail (yellow color) DWs are aligned in -y axis. $H_{a}$ is an external field applied along $+x$-direction to drive the $D W s$.

Fig. 4.11 (a) shows a snapshot of the spatial distribution of spin configuration within the nanowire at $2 \mathrm{~ns}$ after the collision. The magnetic moments are aligned along the wire length, except for a slight canting of the spins near to the collision point. The color contrast along the length of the nanowire indicates that spin waves are emitted and are being propagated towards both ends of the nanowire. The dark color contrast close to the collision point implies that there is a strong perturbance of the magnetization component along the $y$ and $\mathrm{z}$ directions. By comparing the spin configurations at various points in the space, it is observed that magnetization profiles on either side of the collision points are anti- 
symmetric. To better understand the collision phenomenon, various probe points were placed along the width of the NW, on either side of the collision point $(x=0)$. At these points, the temporal evolution of the transverse component of the magnetization, $m_{y}$ was recorded. The total simulation time is $\sim 60 \mathrm{~ns}$. Spectra of SW modes are obtained from the temporal evolution of magnetization via 1D Fast Fourier Transformation (FFT) [27].

(a)

$$
\vec{H}_{a}
$$
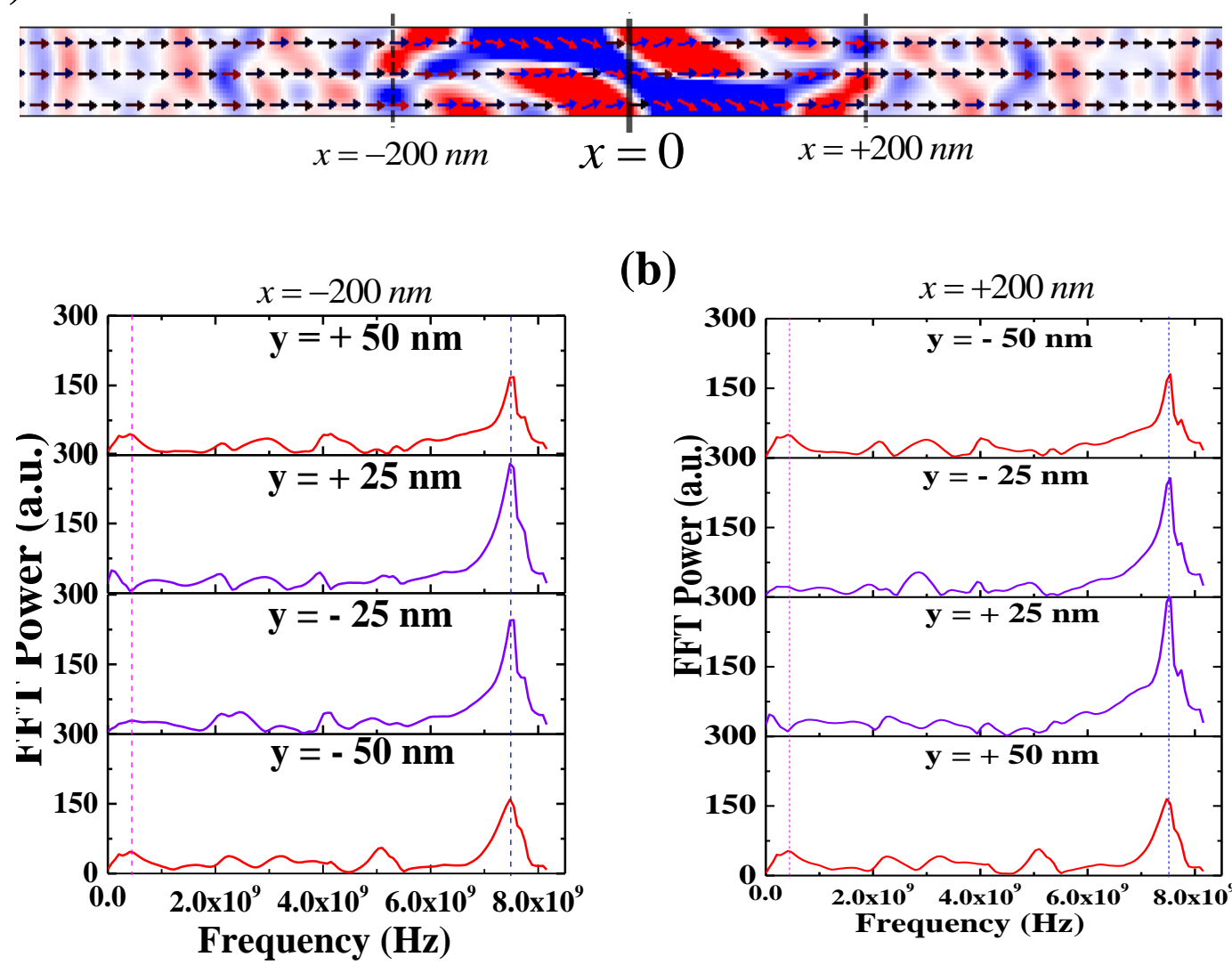

(b)

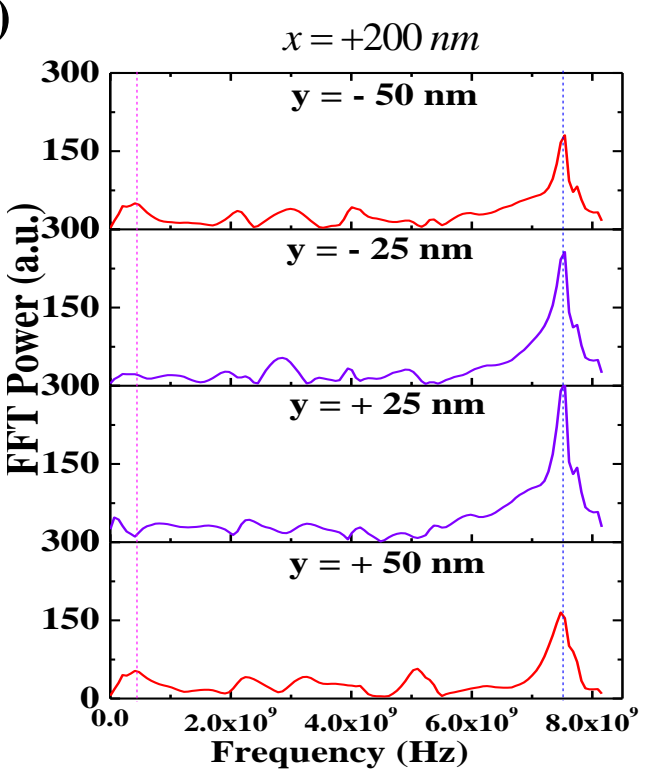

Fig. 4.11 (a) Snapshot image of the spatial distribution of the magnetization profile at $t=2$ $n s$ after the collision. (b) SW spectra obtained from FFTs of $m_{y}(t)$ recorded at various positions along the width of the wire. These probe points are placed at a distance of $x=$ $\pm 200 \mathrm{~nm}$ away from the collision point $(x=0)$. 
A 2D matrix comprises of transverse magnetization $\left(m_{y}\right)$ and simulation time ( $\left.\mathrm{t}\right)$ at these probe points is initially formed. Individual FFTs are then performed at every point along the line, for which the sampling rate is fixed at 60 ps. Fig. 4.11 (b) shows the SW spectra as a function of various probe points positioned at $x=+/-200 \mathrm{~nm}$ from the collision point. A high frequency $(7.5 \mathrm{GHz})$ fundamental mode with wave vector $(k)=0$ exists uniformly along the width.

However, the amplitude of this mode is higher only at the center of the nanowire width $(y=0 \mathrm{~nm})$ and decreases towards the edges of the wire. Besides the fundamental mode, low amplitude propagating modes can also be seen. At the wire edges $(y=+50 \mathrm{~nm}$ and $-50 \mathrm{~nm}$ ), additional modes were formed. These edge modes are due to the inhomogeneity in the profile of internal static magnetic field near the edges of the wire [28]. Due to this, the precession frequency near the edges is different than in the central region. These modes are analogous to the Damon-Eschbach magnetostatic modes appearing in continuous magnetic films [29-31]. It can be clearly seen from Fig. 4.11 (b), the modes at the edge of the nanowire, $x=(+)-200 \mathrm{~nm}$ and $\mathrm{y}=(-)+50 \mathrm{~nm}$, have the same profile of power spectra as at edge $(-)+200 \mathrm{~nm} \& \mathrm{y}=(+)-50 \mathrm{~nm}$. Additionally, the edge modes along width show a diagonally symmetric behavior with respect to the collision point.

From a device perspective, the low amplitude propagating mode will not significantly affect DW dynamics. As such, only fundamental frequency modes were considered. To obtain the decay characteristics of the fundamental mode which arises due to the damping term in LLG equation, individual FFTs are computed at various probe points along $y=0 \mathrm{~nm}$, where the mode has the highest amplitude. 
Shown in Fig. 4.12 are the amplitudes of the power spectra as a function of distance from the collision point. The inset shows the power spectra of the transverse (in-plane, along $y$ axis) and perpendicular (out-of-plane, along $z$-axis) spin wave modes obtained at $x=-200$ $\mathrm{nm}$ and $y=0 \mathrm{~nm}$. Due to lateral confinement of the nanowire, spin wave modes are excited only along $y$ and $z$ - directions. For fixed field and geometrical parameters, SW modes along both orientations have similar frequency; however, the power spectra of the transverse SW is found to be significantly higher than that of the perpendicular SW. So, only the effect of the transverse component of the magnetization was considered the in this work. As seen from Figure 4.12, the spin wave power decays exponentially as it travels along the length of the nanowire.

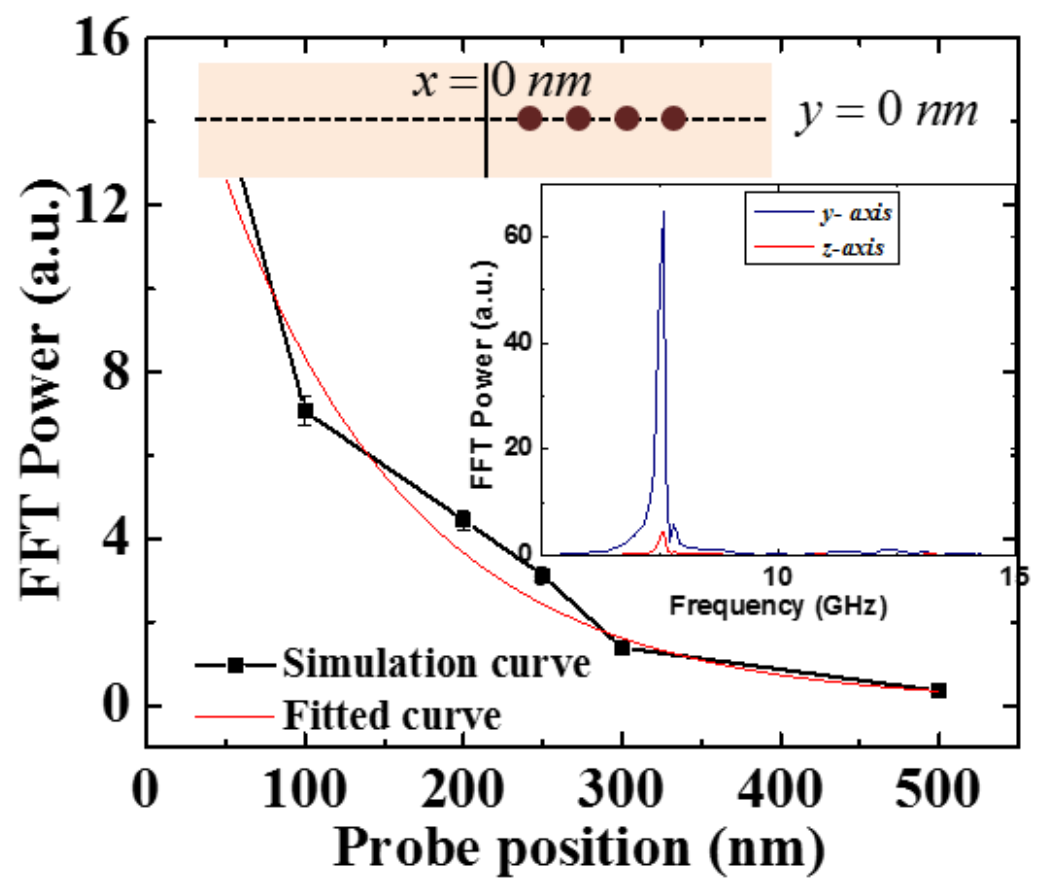

Fig. 4.12 Power spectra of the spin wave modes measured at the probe points along the line $y=0$. Inset shows power spectra corresponding to the transverse $\left(m_{y}\right)$ and perpendicular $\left(m_{z}\right)$ magnetization at $x=-200 \mathrm{~nm}$ and $y=0 \mathrm{~nm}$. 
The curve fitting gives us a decay coefficient of $\beta=98 \mathrm{~nm}$. The curve shows that the spin wave power drops to around to $10 \%$ at a distance of $350 \mathrm{~nm}$ away from the collision point.

\subsubsection{Effect of nanowire width on spin wave characteristics}

To gain an insight into the effect of geometrical parameters on the fundamental SW mode generated during collision, the wire width has been varied from $50 \mathrm{~nm}$ to 150 $\mathrm{nm}$. The upper bound for the width is set by the stability of the transverse DW [32]. Shown in Figure 4.13 (a) are the frequency of the fundamental modes (transverse component) for different wire widths measured along the line $y=0 \mathrm{~nm}$, at a distance of $x=200 \mathrm{~nm}$ from the collision point. The film thickness was kept at $6 \mathrm{~nm}$. A clear red-shift is observed for the fundamental modes as the nanowire width is increased. The inset in Fig. 4.13 (a) shows the FFT power spectra for spin wave mode perpendicular to the wire (z-axis). As can be seen, similar red shift is observed and the frequency of the fundamental modes is consistent with that obtained along the y-axis.
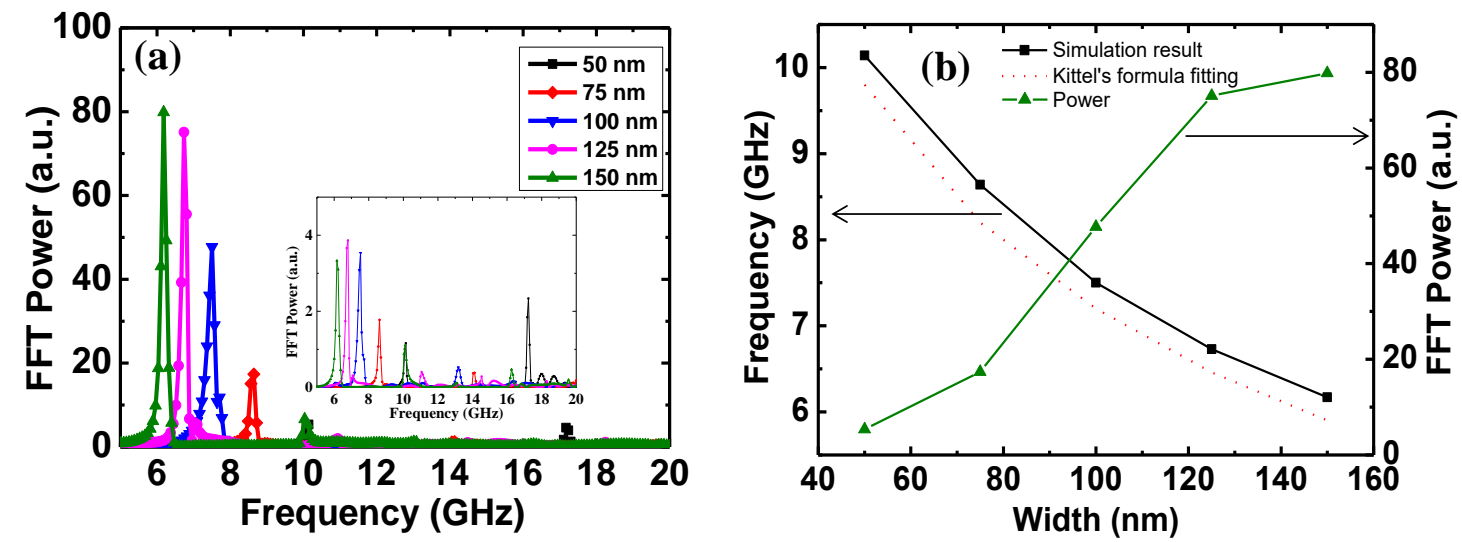

Fig. 4.13 (a) Power spectra of the spin waves for different widths of the nanowire. Inset figure shows power spectra of spin wave obtained from FFTs of $m_{z}(t)$. (b) The frequency and power of the fundamental modes as a function of the width of the nanowire. 
Interestingly, we note that the peak for the $50 \mathrm{~nm}$ wide nanowire is more prominent along the $z$-axis. This is due to the fact that as the wire width reduces the demagnetization field along the width and perpendicular to the plane of the wire becomes comparable, thus the peaks have relatively the same amplitude.

The obtained frequencies for the transverse component are plotted against the Kittel's equation [33-34], which can be used to probe the spin dynamics of a ferromagnetic material:

$$
\begin{gathered}
f=\frac{\gamma}{2 \pi} \sqrt{\left(H_{e f f}+\left(N_{z}-N_{x}\right) \mathrm{M}_{s}\right) \times\left(H_{e f f}+\left(N_{y}-N_{x}\right) M_{s}\right)} \\
N_{x}+N_{y}+N_{z}=1
\end{gathered}
$$

Where $f$ is the frequency of the spin wave, $N_{x}, N_{y}$, and $N_{z}$ are the demagnetization factors along the respective axes. For an infinitely long nanowire, the demagnetization factor along the $x$ axis becomes negligible $\left(N_{x} \sim 0\right)$. The effective field is approximated by the applied field $\left(H_{e f f}=H_{a}\right)$. The Kittel's equation can then be modified as:

$$
f=\frac{\gamma}{2 \pi} \sqrt{\left.H_{a}^{2}+M_{s} H_{a}+N_{y}\left(1-N_{y}\right) M_{s}^{2}\right)}
$$

Shown in Fig. 4.13 (b) is the plot of the frequency of the fundamental mode as a function of the nanowire widths. The obtained frequencies of the fundamental modes from the simulations are shown to match well with those obtained from the Kittel's equation, which is typically used to describe non-propagating spin waves. The DW collision can then be said to excite both the propagating and the non-propagating waves, with the nonpropagating waves possessing the highest amplitude, which can contribute to the dynamics of neighboring DWs [35]. The results also show that the decrease in the spin wave frequency for wider nanowire is accompanied by an increase in the FFT power, which can 
be understood as the result of the system trying to preserve the total energy of the generated spin waves.

\subsubsection{Effect of thickness on spin wave characteristics}

Fig. 4.14 (a) shows the FFT power spectra of the spin wave, measured at $x=200$ $\mathrm{nm}$ and $y=0 \mathrm{~nm}$, as the $\mathrm{Ni}_{80} \mathrm{Fe}_{20}$ film thickness is varied from $1 \mathrm{~nm}$ to $8 \mathrm{~nm}$. The frequency of the fundamental mode is red shifted as the thickness of the nanowire is decreased, which matches well with the Kittel's equation. More importantly, the result shows that the generated spin wave exhibits a second harmonic mode when the nanowire thickness is reduced below $5 \mathrm{~nm}$. At a thickness of $4.5 \mathrm{~nm}$, a second harmonic mode at $f=8.5 \mathrm{GHz}$ is observed, which is approximately 1.2 times the frequency of the fundamental mode. The frequency of the second harmonic increases steadily as the thickness of the nanowire is decreased.
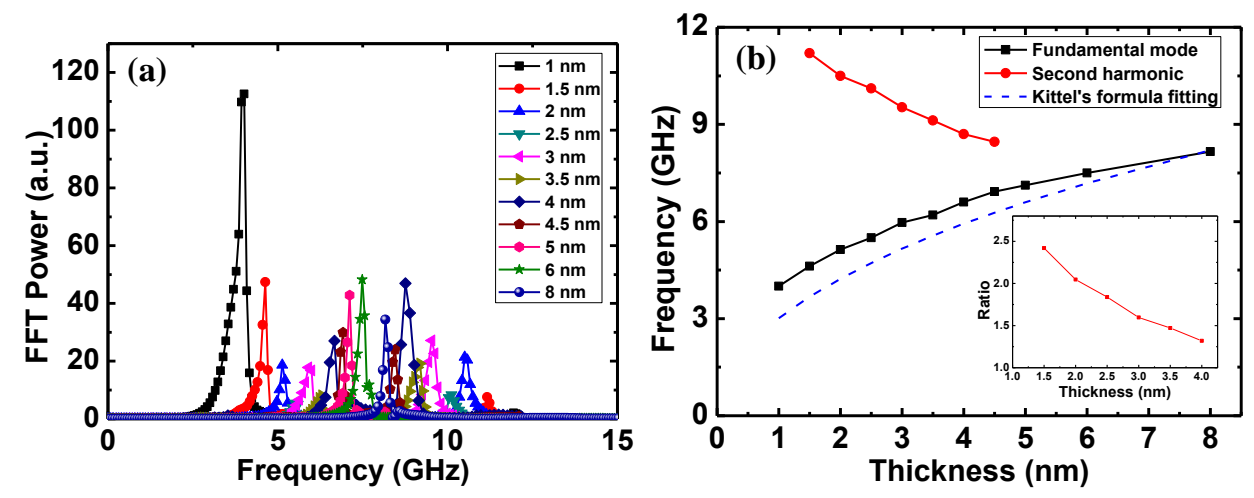

FIG. 4.14 (a) Power spectra for different thicknesses of the nanowire. (b) The frequency of the fundamental and the second harmonic modes as function of the nanowire thickness. Inset figure shows the ratio between the fundamental and second harmonic mode frequencies as a function of thickness of the nanowire. 
For nanowire with $\mathrm{Ni}_{80} \mathrm{Fe}_{20}$ film thickness of $1.5 \mathrm{~nm}$, the frequency of the second harmonic increases to $10.2 \mathrm{GHz}$. The presence of the second harmonic and its dependence on the nanowire thickness cannot be explained by using the Kittel's equation. As the $\mathrm{Ni}_{80} \mathrm{Fe}_{20}$ film thickness is reduced, the ratio of the frequency of the fundamental mode to the second harmonic mode increases, as seen in the inset of Fig. 4.14 (b). This is analogous to the ratio between the fundamental and the second harmonic mode of the one-dimensional infinite quantum well. This result suggests that for thin nanowire, the transverse spin wave can be thought to precess mainly in the $x y$ plane due to the shape anisotropy of the nanowire.

\subsubsection{Effect of in-plane field on spin wave characteristics}

To further understand how the spin waves are affected by the in-plane applied field $\left(H_{a}\right)$, the spin wave power spectra is plotted as a function of different field strengths. As the geometrical parameters are the same for all the simulations, the non-propagating mode displays a fixed frequency as shown in Fig. 4.15.

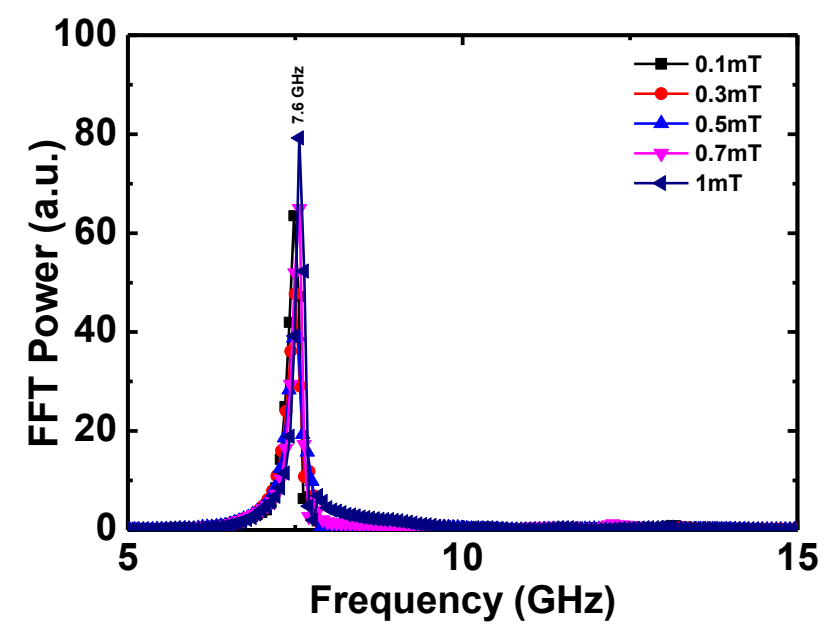

FIG. 4.15 Power spectra of the fundamental mode for different applied field strengths at fixed NW dimensions. 
With the increase of the field strength from $0.1 \mathrm{mT}$ to $1 \mathrm{mT}$, the amplitude of the SW mode is increased. This can be explained by the increase in DW speed as the external field in increase. The higher energy, the DW possess, the more energy that needs to be dissipated during the collision process, resulting in higher amplitude of the fundamental mode.

\subsection{Discussion}

In this work, the random nature of DW generation was ascribed to two phenomena, namely DW nucleation and mutual annihilation of the nucleated DWs [13]. The former can be overcome when the local Oersted field generated by the injection current exceeds a threshold value. From these experiments, these values are $\mathrm{J}=1 \times 10^{12} \mathrm{Am}^{-2}$ and $1.1 \times 10^{12}$ $\mathrm{Am}^{-2}$ with their pulse durations respectively are $\sim 20 \mathrm{~ns}$ and $\sim 10 \mathrm{~ns}$. The later can be overcome if the pair of DWs can be pushed apart by distance of $2.5 \mu \mathrm{m}$ or the DW interaction can be avoided by annihilating one of the DWs [5, 18]. For the deterministic DW generation experiments, the second method was employed and demonstrated a single DW generation with $100 \%$ probability by exploiting the stray field magnetostatic interaction between DWs and the edge of the nanowire. The conventional methods requires 100 pulses to ensure reliable DW generation [24]. By contrast, the new scheme requires single pulse for the deterministic generation of single DW. Additionally, this new scheme eliminates the requirement of long current pulses or external magnetic field to overcome the issue of electric current shunting by the injection line. As such, edge field assisted single DW generation method has shown improvements as regards to the reliability, robustness and lesser current density requirements and hence bringing the on-chip application of DW devices a step forward to actual realization. 


\subsection{Summary}

To summarize, a novel scheme to generate single DW with $100 \%$ probability on a nanosecond time scale was presented. This is achieved by controlling the stray field magnetostatic interaction between DWs and the edge of the nanowire was proposed. The existing techniques require multiple pulses to ensure the reliable DW generation. The proposed edge field assisted single DW generation requires only a single pulse for DW injection with $100 \%$ probability. Also, this scheme eliminates the requirement of long current pulses or external magnetic fields to overcome the issue of current shunting by injection strip line. The field driven DW interaction lead to the mutual annihilation when the topological charges of the two DWs are different. As a result spin waves are emitted and propagated along the nanowire. The spin wave characteristics, such as amplitude and frequency, are found to influence by applied magnetic field and geometrical dimensions of the nanowire. The amplitude of the spin wave modes was found to increase with the increase of field strength. The frequencies of the spin wave modes can be correlated to the nanowire dimensions and independent of the magnetic field strength. The decay coefficient of the spin wave power shows that the power drops to $10 \%$ at a distance of $350 \mathrm{~nm}$ away from the annihilation point. 


\section{References}

1. R. L. Stamps, S. Breitkreutz, J. Akerman, A.V. Chumak, Y. C. Otani, G. E.W. Bauer, J.U. Thiele, M. Bowen, S. A. Majetich, M. Klaui, I. L. Prejbeanu, B.

Dieny, N. M. Dempsey and B. Hillebrands, J. Phys. D: Appl. Phys., 47, 333001 (2014).

2. D. A. Allwood, G. Xiong, C.C. Faulkner and D. Atkinson, Science 309, 1688$1692(2005)$.

3. S. S. P. Parkin, M. Hayashi, and L. Thomas, Science 320, 190-194 (2008).

4. J. Franken, H., H. J. M. Swagten, and B. Koopmans, Nat. Nanotech. 7, 499503(2012).

5. M. Hayashi, L. Thomas, R. Moriya, C. Rettner, and S. S. P. Parkin, Science 320, 209-211(2008).

6. X. Jiang, L. Thomas, R. Moriya, M. Hayashi, B. Bergman, C. Rettner, S.S.P. Parkin, Nat. Comm. 1, 25 (2010).

7. A.Yamaguchi, T. Ono, S. Nasu, K. Miyake, K. Mibu, and T. Shinjo, Phys. Rev. Lett. 96,179904 (2006).

8. M. Hayashi, L. Thomas, C. Rettner, R. Moriya, Y. B. Bazaliy, and S. S. P. Parkin, Phys. Rev. Lett. 98,037204 (2007).

9. G. Meier, M. Bolte, R. Eiselt, B. Kruger, D.H. Kim, and P. Fischer, Phys. Rev. Lett. 98, 187202 (2007). 
10. M. Hayashi, L. Thomas, C. Rettner, R. Moriya, X. Jiang, and S. S. P. Parkin, Phys. Rev. Lett. 97, 207205 (2006).

11. E.R. Lewis, D. Petit, L. O’Brien, A. F. Pacheco, J. Sampaio, A.V. Jausovec, H. T. Zeng, D. E. Read and R. P. Cowburn, Nat.Mater. 9, 980-983 (2010).

12. G. S. D. Beach, Nat. Mater. 9, 959-960 (2010).

13. L.Bocklage, F.U. Stein, M. Martens, T. Matsuyama, and G. Meier, Appl. Phys. Lett. 103, 092406 (2013).

14. J.L. Prieto, M. Munoz and E. Martinez, Phys. Rev. B 83, 104425 (2011).

15. M.Hayashi, L. Thomas, C.Rettner, R. Moriya, and S. S. P. Parkin, Nat. Phys. 3, $21-25$ (2007).

16. M. Munoz, and J.L. Prieto, Nat. Comm. 2, 562 (2011).

17. A. Pushp, T. Phung, C. Rettner, B. P. Hughes, S. E. Yang, L. Thomas and S. S. P. Parkin, Nat. Phys. 9, 505-511 (2013).

18. L.Thomas, M. Hayashi, R. Moriya, C. Rettner, and S. Parkin, Nat. Comm. 3, 810 (2012).

19. F.U .Stein, L. Bocklage, T. Matsuyama, and G. Meier, Appl. Phys. Lett. 100, 192403 (2012).

20. R. Hertel and C. M. Schneider, Phys. Rev. Lett. 97, 177202 (2006).

21. A. Kunz, Appl. Phys. Lett. 94, 132502 (2009). 
22. X. S. Wang, P. Yan, Y. H. Shen, G. E. W. Bauer, and X. R. Wang, Phys.Rev. Lett. 109, 167209 (2012).

23. M. Jamali, H. Yang, and K. J. Lee, Appl. Phys. Lett. 96, 242501 (2010).

24. A. J. Annunziata, . M. C. Gaidis, L. Thomas. Racetrack Memory Cell Array with Integrated Magnetic Tunnel Junction Readout. 2011 IEEE International Electron Devices Meeting(IEDM), Washington DC. IEEE.

DOI:10.1109/IEDM.2011.6131604 (2011, Dec.5-7).

25. M. R. Scheinfein, LLG Micromagnetic Simulator, http://llgmicro.home.mindspring.com/

26. Micromagnetic simulations were performed using the OOMMF code, available at http://math.nist.gov/oommf/. Accessed in June (2013).

27. D. Kumar, O. Dmytriiev, S. Ponraj, and A. Barman, J. Phys. D: Appl. Phys. 44, $235002(2011)$.

28. A. Lara, V. Metlushko, and F. G. Aliev, J. Appl. Phys. 114, 213905 (2013).

29. B. Hillebrands and K. Ounadjela, Spin Dynamics in Confined Magnetic Structure I (Springer-Verlag, Heidelberg, Germany, 2002), pp. 65-89.

30. K. Y. Guslienko, R. W. Chantrell, and A. N. Slavin, Phys. Rev. B 68, 024422 (2003)

31. B. A. Kalinikos and A. N. Slavin, J. Phys. C 19, 7013 (1986).

32. R. D. McMichael and M. J. Donahue, IEEE Trans. Magn. 33(5), 4167 (1997).

33. C. Kittel, Phys. Rev. 73, 155 (1948). 
34. J. B. Yoon, C. Y. You, Y. H. Jo, S. Y. Park, and M. H. Jung, J. KoreanMag. Soc. 20, 1 (2010).

35. N. Zettili, Quantum Mechanics Concepts and Applications (Wiley, Chippenham, Wiltshire, 2009), pp. 215-248. 


\section{Chapter 5}

\section{Spin-orbit torque driven domain wall depinning in $\mathrm{Co} / \mathrm{Ni}$}

\section{heterostructures}

\subsection{Introduction}

This chapter presents chiral Néel domain wall (DW) depinning driven by spinorbit torque (SOT) in perpendicularly magnetized $\mathrm{Co} / \mathrm{Ni}$ nanowires with symmetrical Hall crosses. In these structures, the DW propagation was shown to be opposed in the electron flow direction as evident from the current assisted DW depinning measurements. A transition region from field dominated DW depinning to current dominated DW depinning was established. For field dominated DW depinning, both Up-Down and Down-Up DWs exhibit same depinning fields for different current orientations. However, for current dominated DW depinning, different depinning efficiencies for Up-Down and Down-Up DWs were observed. The difference in depinning fields for Up-Down and Down-Up DWs was found to be increased as the magnitude of the bias current was increased. To reveal the underlying mechanism, current induced SOTs were measured using AC harmonic Hall voltage measurement scheme. The sample exhibits appreciable magnitudes of Slonczewskilike $\left(H_{S L}\right)$ and field-like $\left(H_{F L}\right)$ effective fields. Asymmetric domain expansion under the combination of out-of-plane and in-plane bias fields confirms the existence of sizeable Dzyaloshinskii-Moriya interaction (DMI) that stabilizes left handed chiral Néel DW in the stack. Kerr microscopy was further used for direct observation of the DMI induced DW tilting. The interplay between SOTs and DMI is attributed to the observed depinning anomaly. As the width of the NW was increased, the depinning field was observed to be decreased which can be correlated to decrease in the DW energy. 


\subsection{Motivation}

Current induced domain wall motion (CIDM) in ferromagnetic nanowires has been extensively investigated for the realization of scalable low power non-volatile memory and spin logic devices [1-3]. In bulk ferromagnets, adiabatic spin transfer torque (STT) is responsible for current induced domain wall motion [4-5]. The onset of STT driven DW motion is via the transformation of the internal spin structure from Bloch to Néel [6-7]. However, in ultrathin magnetic heterostructures, a Néel DW can be stabilized by DMI, which is an antisymmetric exchange interaction in a ferromagnetic system arising from the broken inversion symmetry and strong spin-orbit coupling (SOC) [8-12]. It induces an effective field $\left(H_{D M I}\right)$ that promotes chiral Néel DW [13]. Boulle et al. have shown that the DMI results in DW surface tilting in perpendicular magnetized nanowires when the DWs are driven by out-of-plane fields or spin polarized currents. The degree of tilting depends on the strength of DMI [14-16]. Additionally, the strong SOC in these materials also generate current induced spin-orbit torques through spin Hall effect (SHE) and Rashba effect (RE) [17-24]. These effects produce Slonczewski-like and field-like torques. The effective field from Slonczewski-like torque is phenomenologically equivalent to an applied out-of-plane field that induces DW motion. The effective field from field-like torque which lies in-plane and transverse to the current direction, does not contribute to the DW dynamics but it can alter the DW internal magnetization [25]. The detection of DW in perpendicularly magnetized nanowires has been performed via Hall cross structures [26]. Such structures introduce potential landscapes for the DW propagation. There have been several experimental reports on pinning and depinning of the DW driven by bulk STT [27]. But 
SOT driven DW depinning in ultrathin NWs is still elusive. In this work, SOT assisted chiral Néel DW depinning from Hall crosse was reported.

\subsection{Current assisted domain wall depinning through Hall cross}

\subsubsection{Experimental setup}

As shown in Fig. 5.1 (a), the thin film stack of Ta (5)/Pt (5)/[Co $(0.25) / \mathrm{Ni}(0.5)]_{4} / \mathrm{Co}$ $(0.25) / \mathrm{Ru}(5)$ was deposited on thermally oxidized $\mathrm{Si}$ wafer $\left(300 \mathrm{~nm}\right.$ thick $\left.\mathrm{SiO}_{2}\right)$ by $\mathrm{DC}$ magnetron sputtering. The figures in parentheses represent the layer thicknesses in $\mathrm{nm}$ and the subscript denotes the number of repetitions. All the layers were deposited at room temperature under $2.5 \mathrm{mT}$ Torr Ar at a base pressure of $\sim 2 \times 10^{-7}$ Torr. To break the structural inversion symmetry, two different heavy metal layers, Pt and Ru, were deposited as seed and capping layers respectively. Fig. 5.1 (b) shows in-plane and out-of-plane magnetizations versus external magnetic field as obtained from alternating gradient field magnetometer. It can be clearly seen that the sample has an out-of-plane easy axis. The estimated saturation magnetization $\left(M_{s}\right)$ and effective anisotropy constant $K_{\text {eff }}$ are 362 $\mathrm{emu} / \mathrm{cc}$ and of $1.24 \times 10^{6} \mathrm{erg} / \mathrm{cc}$ respectively.

Nanowires with two symmetrical Hall crosses were patterned using electron beam lithography and ion milling techniques from the thin film stack. Fig. 5.2 shows schematic of the device with measurement setup. Two Ta (5)/Cu (80)/Au (20) electrodes labelled A and $\mathrm{B}$ were patterned on top of the nanowire to nucleate and drive the DW, respectively. Before depositing the electrodes, the surface of the ferromagnetic structure is slightly etched using reverse sputtering to promote better electrical contacts. 

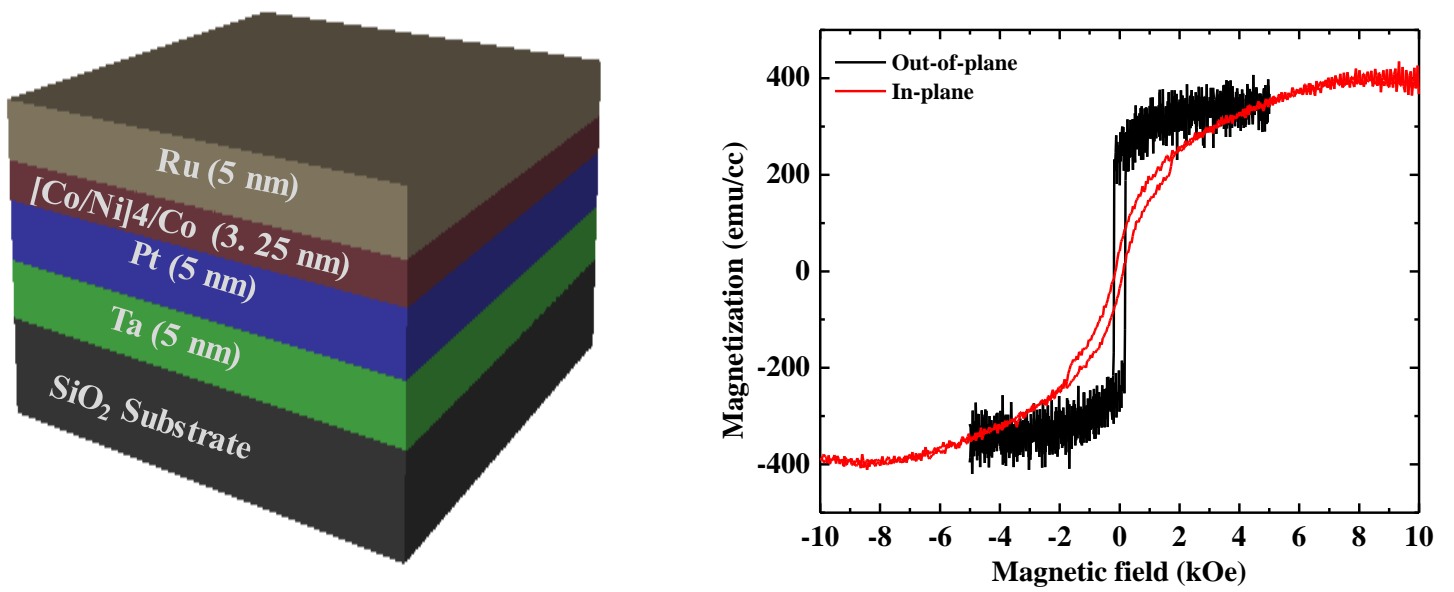

Fig. 5.1 (a) Schematic of Ta (5)/Pt (5)/[Co (0.25)/Ni (0.5)] $/ / \mathrm{Co}(0.25) / \mathrm{Ru}$ (5) stack structure. (b) In-plane and out-of-plane magnetization curves for the stack. The stack shows an out-of-plane easy axis.

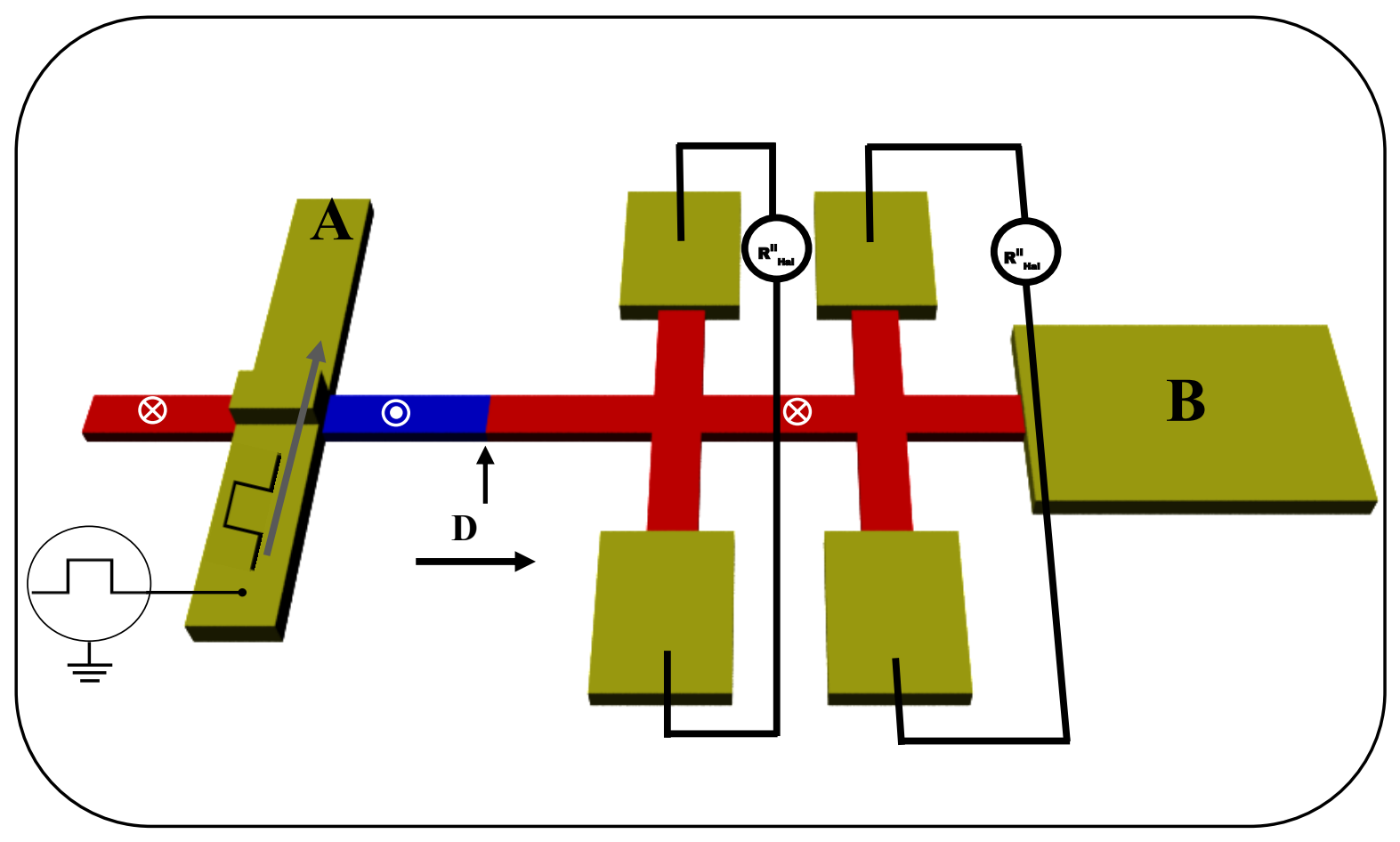

Fig. 5.2 Schematic of the device structure with measurement setup. It comprises of a $\mathrm{Co} / \mathrm{Ni}$ $N W$ with two Hall crosses labelled as $H C_{I}$ and $H C_{I I}$ and two Ta/Cu/Au electrodes $A$ and $B$. 
For DW nucleation, a pulse current of $80 \mathrm{~mA}$ with $50 \mathrm{~ns}$ duration was injected through electrode A. A DC bias current $\left(I_{D C}\right)$ was injected via bias tee, which separates DC current source from a pulse generator. An out-of-plane field $\left(H_{z}\right)$ together with DC bias current was employed to drive the DW. The DW displacement was detected at the Hall cross through anomalous Hall effect (AHE), which is proportional to the component of magnetization perpendicular to the NW. All measurements in this work were carried out at room temperature.

Fig. 5.3 (a) shows the SEM image of a device structure with a $300 \mathrm{~nm}$ wide nanowire and two Hall crosses separated by a distance of $3 \mu \mathrm{m}$. The normalized Hall resistance $\left(R_{\text {Hall }}\right)$, without any DW injected, while an external magnetic field is swept along the $z$-axis, is shown in Fig 5.3 (b).

(a)

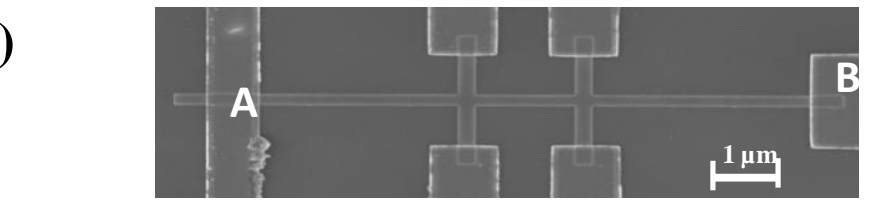

(b)

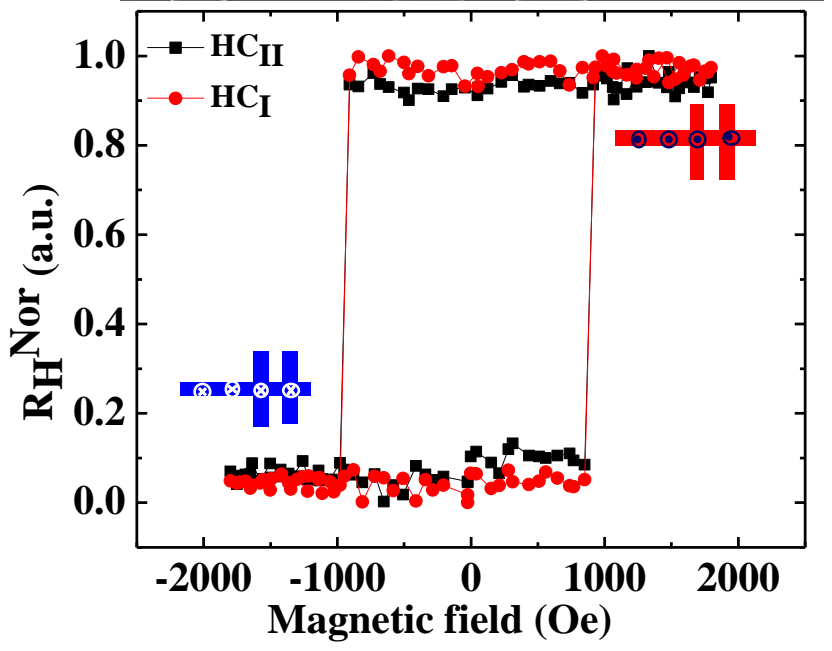

Fig. 5.3 (a) SEM image of a $30 \mathrm{~nm}$ wide $N W$ with two Hall crosses separated by a distance of $3 \mu \mathrm{m}$. (b) Normalized Hall resistance $\left(R_{H}{ }^{\text {Nor }}\right)$, without any DW injected, as a function of external magnetic field applied along the out-of-plane direction. 
The normalized $\mathrm{R}_{\text {Hall }}$ value of 1 (0) corresponds to an upward, $+z$ (downward, $-z$ ) orientation of magnetization in the Hall cross. A clear square hysteresis loop with a coercive field of $\sim 900$ Oe confirms that the magnetic easy axis of the nanowire is perpendicular to the plane of the nanowire. Additionally, the same coercive field was obtained at both $\mathrm{HC}_{\mathrm{I}}$ and $\mathrm{HC}_{\mathrm{II}}$ which confirms that the two Hall crosses are behaving identically.

\subsubsection{Domain wall injection and detection}

DW depinning measurement involves the DW injection, propagation and detection. Local Oersted field generation method was employed to inject the DW. Initially, a perpendicular magnetic field $\left(H_{z}\right)$ of $\sim+2 \mathrm{kOe}$ was applied globally to obtain a single magnetization state along the $+z$ (Up) orientation. A current pulse is then injected through electrode A. It results in the formation of a $\downarrow \uparrow \mathrm{DW}$ on the right edge of the electrode. Similarly, a $\uparrow \downarrow$ DW can also be injected by setting the initial magnetization of the NW along the $-z$ (down) orientation. After the DW has been generated, the application of an external out-of-plane field in opposite direction $(-z)$ drives the DW towards the Hall cross. Fig 5.4 shows the normalized $\mathrm{R}_{\text {Hall }}$ measured across the Hall cross when the magnetic field was scanned from 0 to -350 Oe while a DC bias current, IDC, of $100 \mu \mathrm{A}$ was applied from electrode A to B.

The critical field $\left(\mathrm{H}_{\mathrm{dep}}\right)$ required for the DW to overcome the potential barrier at the Hall cross can be inferred from the AHE measurement. Two jumps in $\mathrm{R}_{\text {Hall were }}$ observed at about an external field of -96 Oe and -156 Oe, which corresponds to a field at which DW reaching the Hall cross and depinning field of the DW from the Hall cross respectively. 


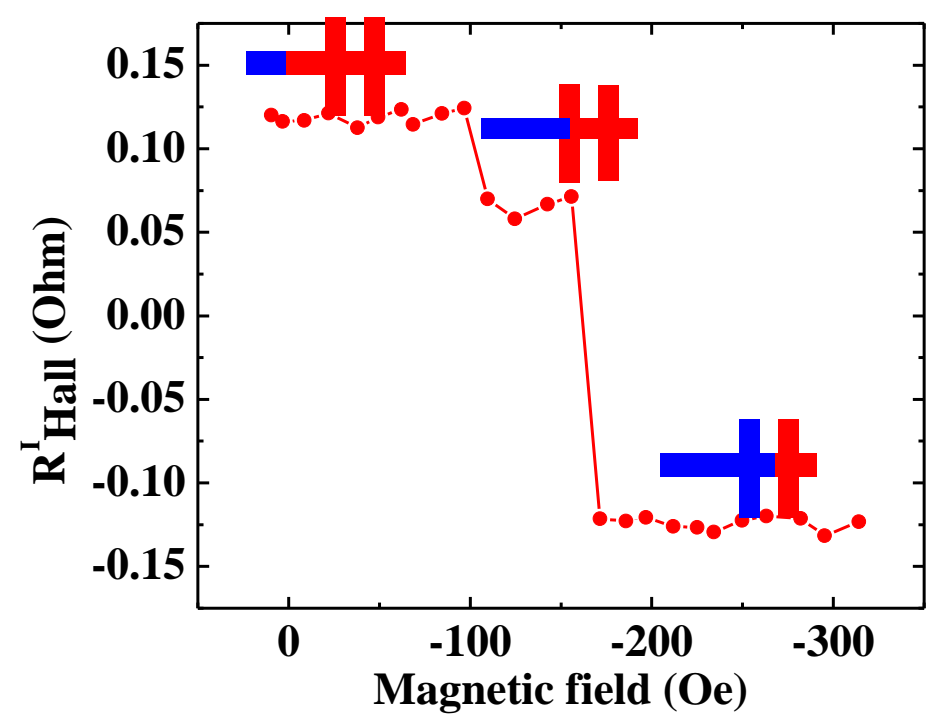

Fig. 5.4 Hall resistance measured across $H C_{I}$, after the injection of a $D W$. The switching field of 156 Oe corresponds to DW depinning field $\left(H_{d e p}\right)$.

\subsubsection{Current assisted domain wall depinning}

To understand the effect of DC bias current on the DW depinning process, DC currents with varying amplitudes were applied from $\mathrm{A} \rightarrow \mathrm{B}$ and $\mathrm{B} \rightarrow \mathrm{A}$. For currents applied from $\mathrm{A} \rightarrow \mathrm{B}(\mathrm{B} \rightarrow \mathrm{A})$, the direction of the current is parallel (anti parallel) to the DW motion. Note that the direction of the DW motion, from electrode A to B, is governed solely by the external out-of-plane field while $I_{D C}$ assists or opposes the DW motion. The maximum value of $I_{D C}$ was limited to $900 \mu \mathrm{A}$ so as to negate any thermal effects that may influence the DW motion. The corresponding depinning fields, $H_{\text {dep }}$, measured for each DC bias current is plotted in Fig 5.5. $H_{d e p}$ varies linearly as a function of $I_{D C}$ for both directions of the current flow but they exhibit opposite slopes as a function of the magnitude of the current. When $I_{D C}$ is applied from B $\rightarrow \mathrm{A}$, the magnitude of $H_{d e p}$ increases with $I_{D C}$. However, an opposite trend was obtained for $I_{D C}$ applied from A $\rightarrow$ B. 


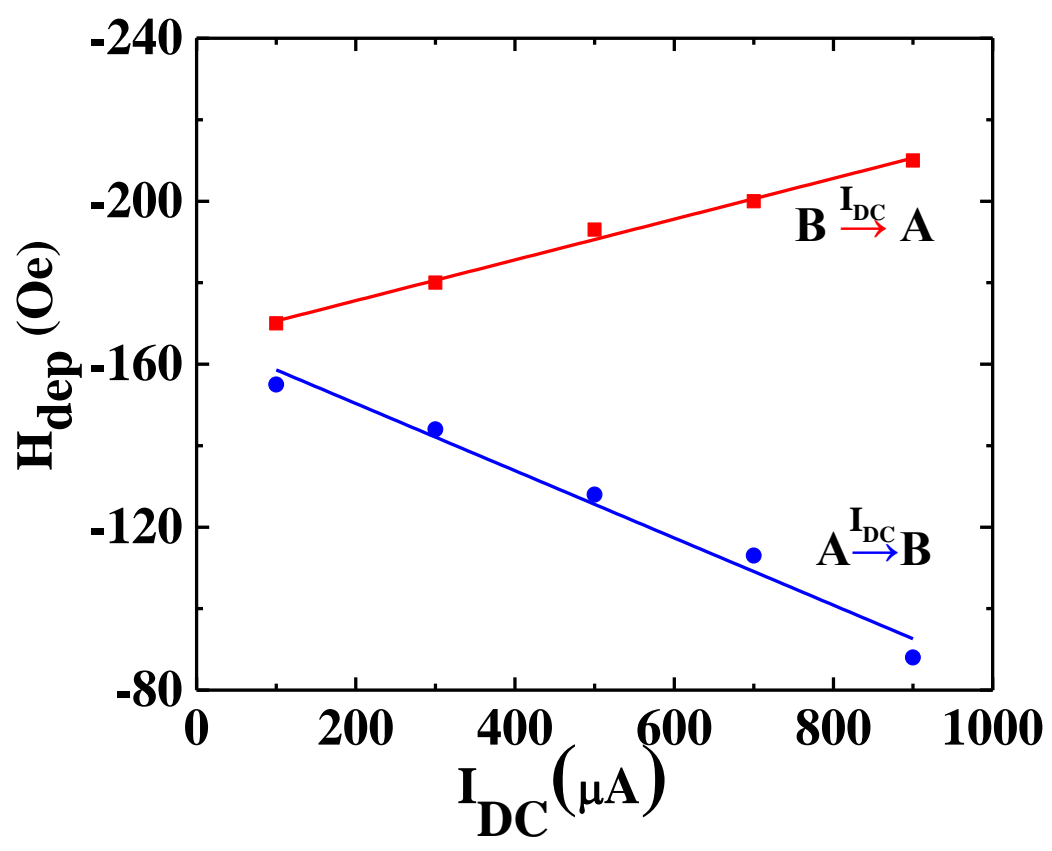

Fig. 5.5 Linear dependence of $D W$ depinning field $\left(H_{\text {dep }}\right)$ as a function of DC bias current $\left(I_{D C}\right)$ directed from $A \rightarrow B$ and $B \rightarrow A$. Solid lines represent the fitted linear curves.

The observed results suggest that the DW motion is being assisted along the current flow direction. The strength of the intrinsic pinning barrier of $\mathrm{Co} / \mathrm{Ni}$ nanowire was obtained by extrapolating $H_{d e p}$ to $I_{D C}=0$ and was estimated to be $\sim 165$ Oe. The slope of $H_{d e p}$ vs $j_{e}$ gives the depinning efficiency $\left(\left|\Delta H_{\text {dep }} /\right| \Delta J \mid\right)$ and is computed to be $\sim 15$ and $\sim 25$ Oe per $10^{11}$ $\mathrm{Am}^{-2}$ for currents applied from $\mathrm{B} \rightarrow \mathrm{A}$ and $\mathrm{A} \rightarrow \mathrm{B}$, respectively. The increase in $\mathrm{H}_{\text {dep }}$ with the increase of $I_{D C}(B \rightarrow A)$ cannot be explained with the prediction of the conventional bulk STT, where one would expect the lowering of the $H_{\text {dep }}$ for the DW moving in the direction of the electron flow (B $\rightarrow$ A). Instead, an alternative mechanism whereby DMI induced chiral Néel DW driven by spin-orbit torques may account for these observed results. Joule heating does not influence the DW depinning process in this experiment. For the whole range of DC bias currents investigated, the temperature of the nanowire was 
monitored by measuring the resistance of the nanowire. The wire resistance did not exhibit any appreciable change implying that the joule heating effect is negligibly small in our sample. Additionally, if joule heating effect were present, irrespective of the direction of $I_{D C}$, a lower depinning field and a deviation from the linear behavior of the current dependent $H_{d e p}$ would have been observed.

\subsubsection{Estimation of the strength of DMI effective field}

To confirm that chiral DW structures are indeed stabilized in our NW, the strength of the DMI effective field in our sample stack was evaluated. It was measured by simple field induced asymmetric domain expansion method [13]. This method of DMI measurement eliminates any complexities arise from current induced effects such as spintransfer, spin Hall and Rashba effects. The DW expansion was observed by a polar Kerr microscope equipped with two electromagnets that generate both in-plane and out-of-plane magnetic fields. In the absence of an in-plane field, $H_{x}=0$, the domain expansion is expected to be symmetric with respect to an axis parallel to the out-of-plane field as shown in Fig. 5.6 (a). The symmetry is broken when an in-plane field is applied in combination with the out-of-plane field. Figs. $5.6(\mathrm{~b}, \mathrm{c})$ show the images of the domain expansion driven by a fixed out-of-plane field of 105 Oe under an in-plane bias field with varying amplitudes pointing in $-x$ direction. Figs. $5.6(\mathrm{~d}, \mathrm{e})$, correspond to in-plane fields applied along $+x$ direction respectively. Blue arrow represents the direction in which the magnitude of the in-plane field is increasing. As can be seen from Fig. $5.6(\mathrm{~b}, \mathrm{c})$, the right edge of the domain

is moving at a faster rate as compared to the left edge when the in-plane field is oriented along $-x$ direction. 


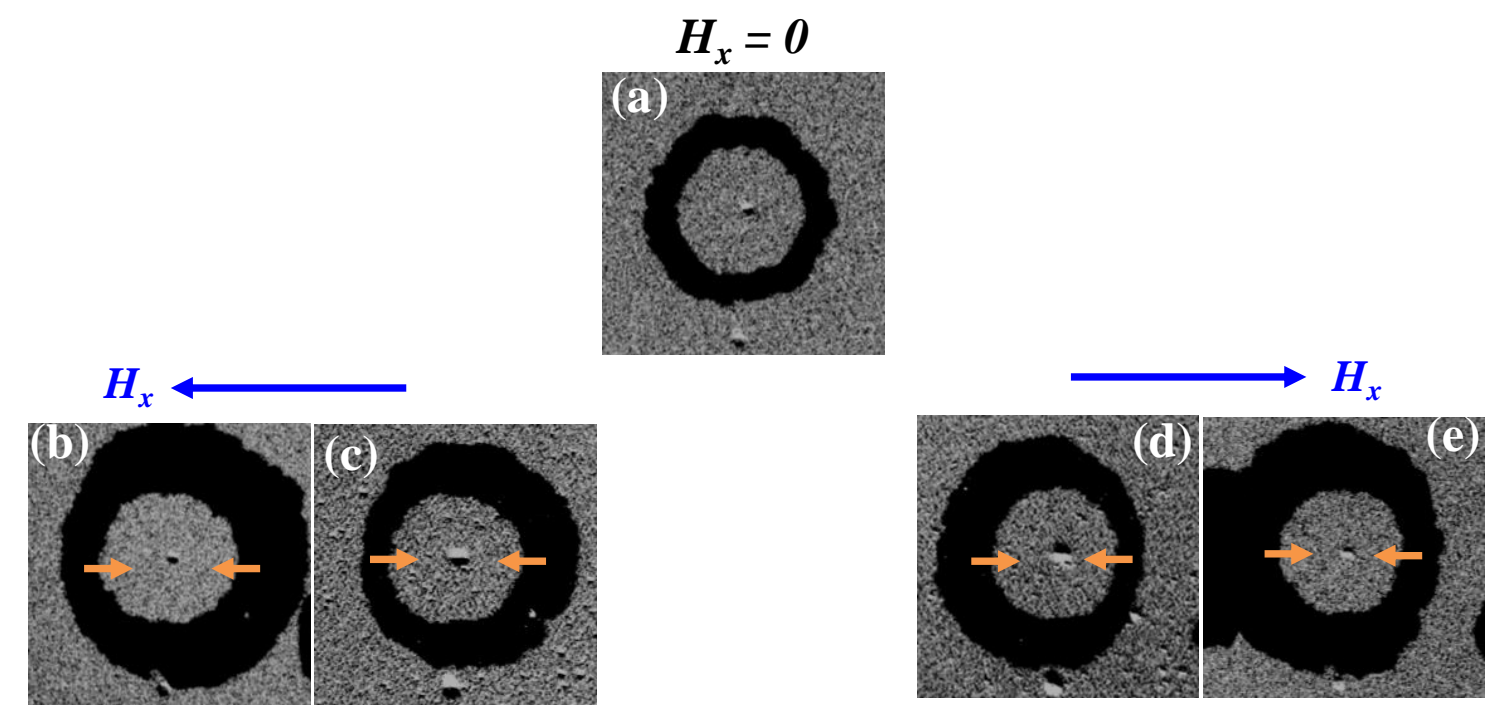

Fig. 5.6 (a) Differential Kerr images of a field $\left(H_{Z}\right)$ driven domain expansion in the absence of in-plane field $\left(H_{x}=0\right) .(b, c)$ asymmetric domain expansion with in-plan field applied along -x axis. $(d, e)$ asymmetric domain expansion with in-plane applied along $+x$-axis. Dark contrast represents a Up domain while arrows on the domain indicate the equilibrium magnetization direction within the $D W$.

The asymmetric expansion of the domain confirms that the magnetization inside the DW rotates anti-clockwise with left handed chirality and hence DMI is negative. To quantify the asymmetry in the domain expansion, the velocities of left and right edges of the domain were plotted as a function of in-plane field $\left(H_{x}\right)$. As shown in Fig. 5.7, the two velocity plots shift away from $H_{x}=0$ in opposite directions. The field at which the minimum velocity occurs, $\sim 45 \mathrm{mT}$ in our sample, provides an estimate of the DMI field, $\mathrm{H}_{\mathrm{DMI}}=-45$ mT. The in-plane field dependence of the DW energy [10] can be expressed as

$$
\sigma\left(H_{x}\right)=\sigma_{0}+2 K_{D} \Delta-\pi \Delta \mu_{0} M_{s}\left|H_{x}+H_{D M I}\right|,
$$


where $M_{s}$ is the saturation magnetization, $\sigma_{0}$ is the Bloch wall energy density, $\Delta$ is the DW width, $K_{D}$ is the DW anisotropy energy.

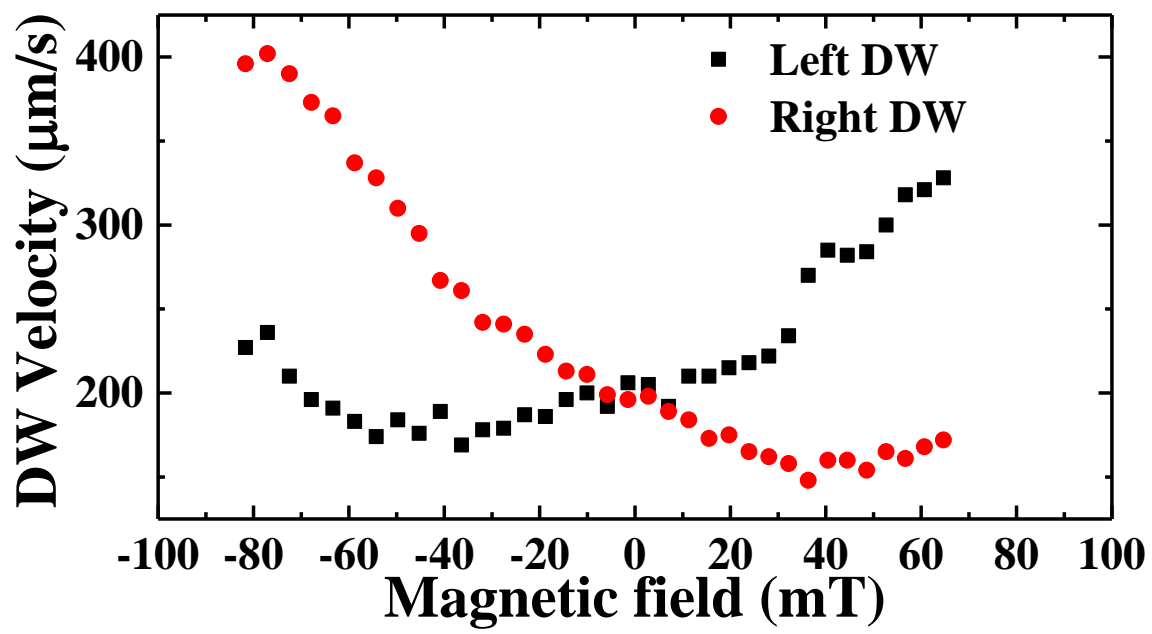

Fig. 5.7 DW velocity as a function of in-plane bias field for left (black dots) and right (red dots) DWs.

In case when the DW is fully transformed into a Néel wall, then

$$
\left|H_{x}+H_{D M I}\right|>4 K_{D} / \pi \mu_{0} M_{s} \equiv \mu_{0} H_{N-B} .
$$

The measured parameters for our thin film are; $M_{s}=3.6 \times 10^{5} \mathrm{~A} / \mathrm{m}, H_{k}=7 \mathrm{kOe}$, $K_{0}=\mu_{0}\left(H_{K} M_{s}-M_{s}^{2}\right) / 2=1.24 \times 10^{5} \mathrm{~J} / \mathrm{m}^{3}, \Delta=\sqrt{A K_{0}}=10 \mathrm{~nm}, \sigma_{0}=2 \pi \sqrt{A K_{0}}=8 \mathrm{~mJ} / \mathrm{m}^{2}$ and $K_{D}=N_{x} \mu_{0} M_{s}^{2} / 2=2.2 \times 10^{4} J / m^{3}$, where $N_{x}=t_{F} \log 2 /(\pi \Delta)=0.07$ is the demagnetizing coefficient of the wall [28] and $t_{F}$ being the ferromagnet film thickness. The exchange stiffness constant $(A)$ of $15 \mathrm{pJ} / \mathrm{m}^{2}$ was considered in our calculations. The critical field separating the Néel wall from the Bloch wall was calculated to be $\mu_{0} H_{N-B}=20 \mathrm{mT}$. As the magnitude of the measured $H_{D M I}(-45 \mathrm{mT})$ in the film is much larger than $H_{N-B}(20$ 
mT), the Néel DW configuration is favored. The negative sign of $\mathrm{H}_{\mathrm{DMI}}$ implies that the Néel DW adopts a stable structure with left handed chirality. The estimated effective DMI constant is $D=\mu_{0} H_{D M I} M_{s} \Delta=0.16 \times 10^{-3} \mathrm{~J} / \mathrm{m}^{2}$ [10].

For our thin film stack, the magnetic multilayer $[\mathrm{Co} / \mathrm{Ni}]_{4} / \mathrm{Co}$ is sandwiched between two heavy metal layers of $\mathrm{Pt}(5 \mathrm{~nm})$ and $\mathrm{Ru}(5 \mathrm{~nm})$. The spin Hall angle $\left(\theta_{S H E}\right)$ of $\mathrm{Ru}$ is negligibly small and has positive sign [29]. As such, the significant source of spin current is expected to be from the Pt layer. Since Pt has positive spin Hall angle $\theta_{S H E}>0$, the lefthanded Néel DW is expected to move along the current flow direction. This is consistent with our observed experimental results from the DMI measurement.

\subsubsection{Quantification of current induced effective fields}

To quantify the strength of SOT induced effective fields in our sample, AC harmonic Hall voltage measurement scheme was employed [30]. To measure the effective fields, the $\mathrm{Co} / \mathrm{Ni}$ stack was patterned into $1.5 \mu \mathrm{m}$ wide Hall cross structure using electron beam lithography and ion milling. The variations of the in-phase first harmonic and out-of phase second harmonic Hall voltages with in-plane field are used to compute the Slonczewski-like $\left(\mathrm{H}_{\mathrm{SL}}\right)$ and the field-like $\left(\mathrm{H}_{\mathrm{FL}}\right)$ effective fields generated, respectively, by Slonczewski-like and field-like and torques.

The schematic of the measurement set-up for characterizing the Slonczewski like $\left(H_{S L}\right)$ and field like $\left(H_{F L}\right)$ effective fields are shown in Fig. 5.8 (a, b) respectively. An AC current with a fixed frequency was passed through the nanowire and an in-plane field was simultaneously swept along longitudinal $\left(H_{x}\right)$ or transverse $\left(H_{y}\right)$ to the nanowire. They successively generate Slonczewski like $\left(H_{S L}\right)$ and field like $\left(H_{F L}\right)$ effective fields. The 
injected AC current generates alternating effective fields, causing the perpendicular component of the magnetization $\left(m_{z}\right)$ of ferromagnet to oscillate with a driving frequency, $\omega$. The AC induced effective fields are given by the following equation [30]

$$
H_{S L(F L)}=-2 \frac{\partial V_{2 \omega} / \partial H_{x(y)}}{\partial^{2} V_{\omega} / \partial H_{x(y)}^{2}},
$$

where $V_{\omega}$ and $V_{2 \omega}$ are the first and second harmonics of the Hall voltage, measured by a lock-in amplifier. The excitation frequency of $330 \mathrm{~Hz}$ with the in-plane field was swept between \pm 600 Oe.

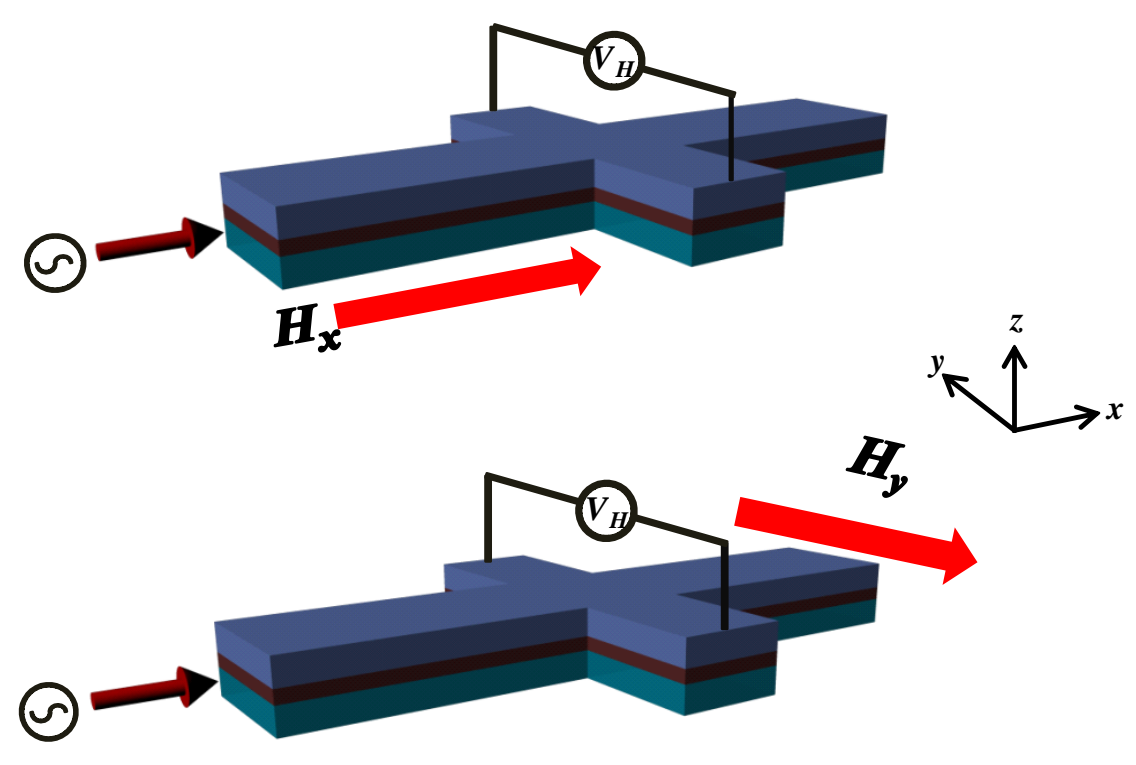

Fig. 5.8 Schematic of the measurement setup for Slonczewski-like $\left(H_{S L}\right)$ effective field $(a)$ and field-like $\left(H_{F L}\right)$ effective field $(b)$. The AC current was always applied along the NW long axis and Hall voltage was measured across the Hall bar. 
Fig. 5.9 (a) shows the $V_{\omega}$ as a function of longitudinal field $\left(H_{x}\right)$ at a fixed current density of $3.5 \times 10^{11} \mathrm{~A} / \mathrm{m}^{2}$ for both Up and Down magnetized states of the NW. The curves for Up and Down magnetized states are symmetrical with respect to x-axis except that they have opposite signs. A polynomial fitting was performed on the curve corresponding to the Up magnetized state that gives an equation of the form

$$
V_{\omega}=A_{1} H_{x}+A_{2} H_{x}^{2}
$$

where $A_{1}$ and $A_{2}$ are polynomial coefficients of the equation.

(a)

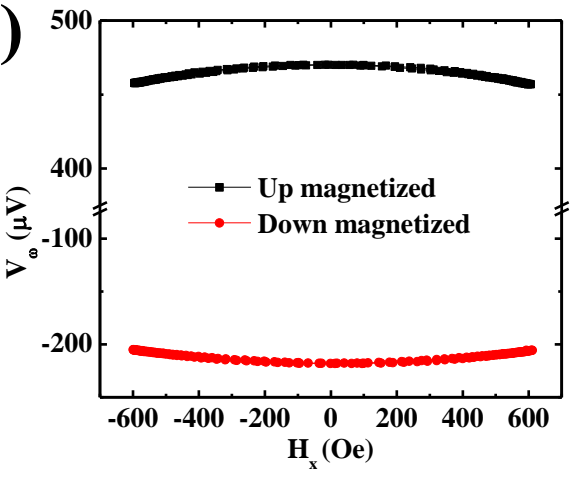

(c)

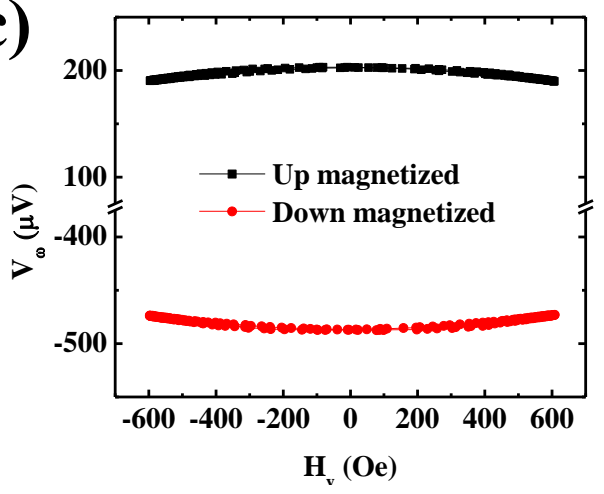

(b)

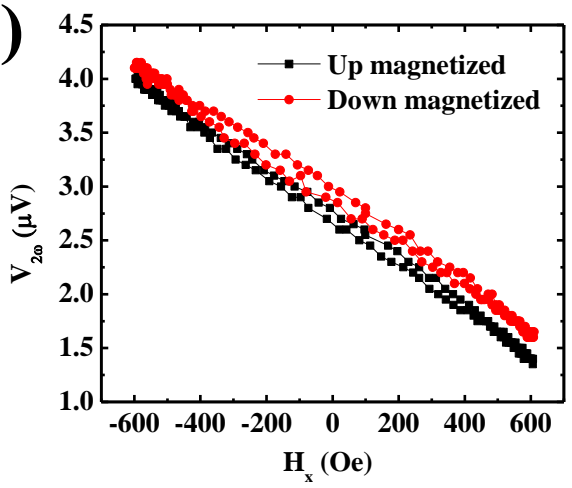

(d)

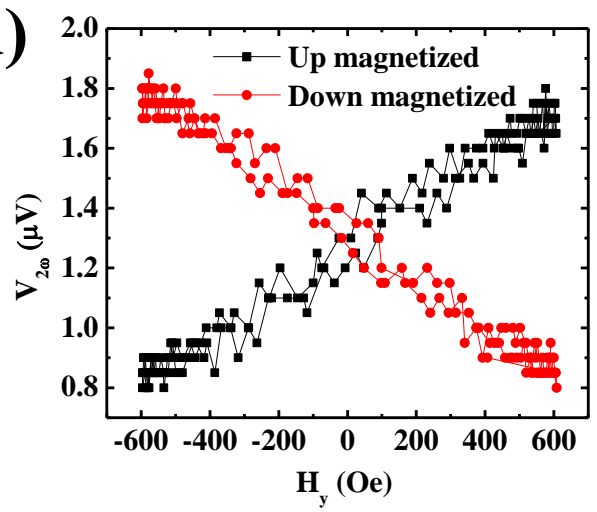

Fig. $5.9 V_{\omega}(a)$ and $V_{2 \omega}(b)$ as a function of longitudinal field $\left(H_{x}\right)$ for both Up and Down magnetized states of the nanowire. $V_{\omega}(c)$ and $V_{2 \omega}(d)$ as a function of transverse field $\left(H_{y}\right)$. 
Similarly, $V_{2 \omega}$ was measured with the same experimental setup and Fig. 5.9 (b) shows the in-plane field $\left(H_{x}\right)$ dependence of $V_{2 \omega}$ at a fixed current density of $3.5 \times 10^{11}$ $\mathrm{A} / \mathrm{m}^{2}$ for both Up and Down magnetized states of the nanowire. A linear fitting was performed for $+M_{z}$ to get an equation of the form

$$
V_{2 \omega}=B_{1} H_{x}+B_{2}
$$

where $\mathrm{B}_{1}$ and $\mathrm{B}_{2}$ represent respectively the slope and intercept of the linear curve. The Slonczewski-like effective field $\left(\mathrm{H}_{\mathrm{SL}}\right)$ can be obtained by substituting the eqns. (5.4) \& (5.5) into eqn. (3) as follows

$$
H_{S L}=-\frac{B_{1}}{A_{2}}
$$

Similar relation exists for $\mathrm{H}_{\mathrm{FL}}$, when $\mathrm{V}_{\omega}$ and $\mathrm{V}_{2 \omega}$ were measured with in-plane field applied transverse to the NW axis. Figs. 5.9 (c, d) show the representative plots for $\mathrm{V}_{\omega}$ and $\mathrm{V}_{2 \omega}$ as a function of in-plane field $\left(\mathrm{H}_{\mathrm{y}}\right)$ for both $\mathrm{Up}$ and Down magnetized states. For both longitudinal and transverse field sweep, $\mathrm{V}_{\omega}$ shows similar trend for $\mathrm{Up}$ and Down magnetization configurations. However, the variation of $\mathrm{V}_{2 \omega}$ with $\mathrm{H}_{\mathrm{y}}$ exhibit opposite signs of slopes for Up and Down magnetizations of the NW.

The SOT induced effective fields are then extracted using equation (5.3) and vary linearly with $J_{e}$, as shown Figs. $5.10(\mathrm{a}, \mathrm{b})$. The Slonczewski-like effective field, $H_{S L}$ was computed to be $\sim 15$ Oe per $10^{11} \mathrm{~A} / \mathrm{m}^{2}$ and the field-like field, $H_{F L}$ was 7 Oe per $10^{11} \mathrm{~A} / \mathrm{m}^{2}$. 

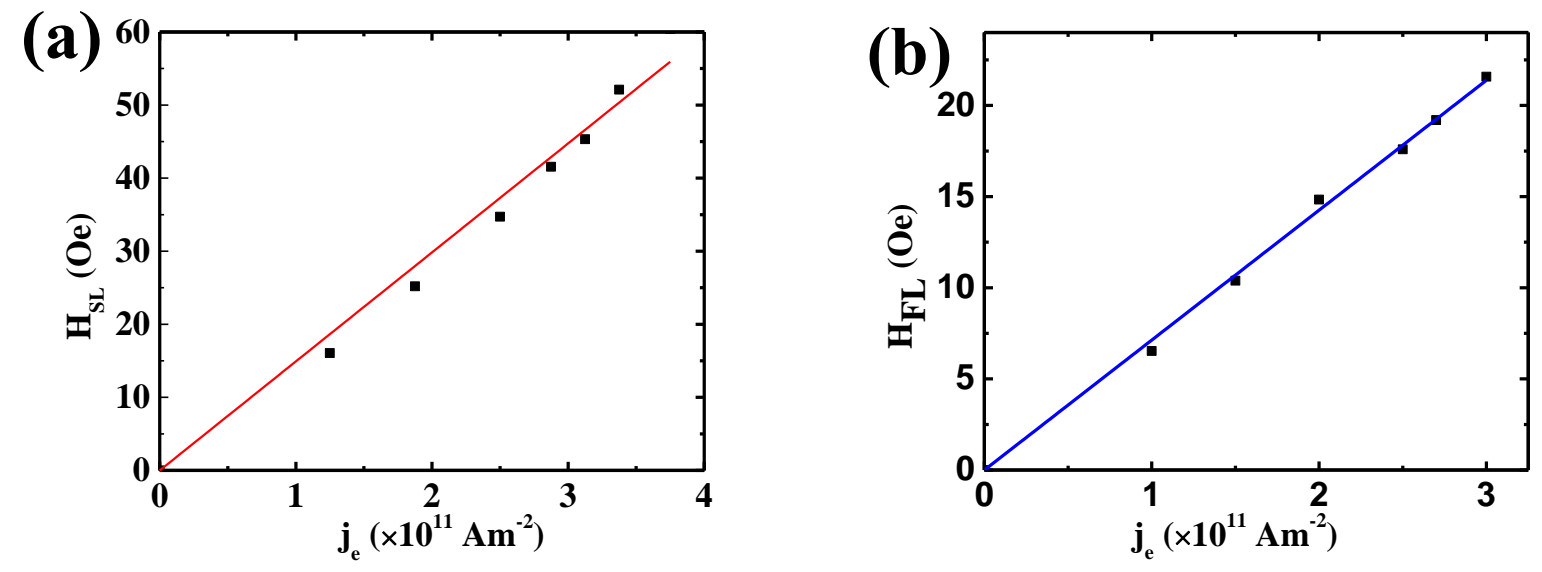

Fig. 5.10 Current induced effective fields $\left(H_{S L}\right)$ and $\left(H_{F L}\right)$ as a function of AC current density $\left(j_{e}\right)$ applied longitudinal $(a)$ and transverse $(b)$ to the $N W$. Solid lines are the fitted curves.

The DMI measurement concluded that the effective DMI $\left(H_{D M I}\right)$ in the film stack structure stabilizes a Néel DW configuration with left handed chirality while the SOT measurement reveal the presence of appreciable magnitudes of Slonczewski-like term $\left(H_{S L}\right)$ and field-like torques due to SOT.

\subsubsection{Effect of DMI and SOT on the domain wall depinning}

To further investigate the effect of DMI and SOT on the DW dynamics for both $\uparrow \downarrow$ and $\downarrow \uparrow$ DWs, the depinning experiments were repeated in a $500 \mathrm{~nm}$ wide nanowire. In order to move both $\uparrow \downarrow$ and $\downarrow \uparrow$ DWs in the same direction for the detection at the Hall cross, the sign of the $H_{z}$ field was reversed and the current was passed along the direction of the field induced DW propagation.

The depinning fields are plotted as a function of the bias current as shown in Fig 5.11 (a). As expected, for currents flowing along the DW propagation direction $(A \rightarrow B)$, the depinning field reduces linearly as the magnitude of the current is increased for both $\uparrow \downarrow$ 
and $\downarrow \uparrow$ DWs. Conversely, for DW motion opposing the current flow $(\mathrm{B} \rightarrow \mathrm{A})$, the depinning field increases monotonically as a function of the magnitude of the current. As Pt has a positive spin hall angle, SOT induced DW motion will be along the direction of the applied current. For $I_{D C} \leq 300 \mu \mathrm{A}$, magnitude of the depinning fields for different directions of the current and different types of DWs are almost the same. However, for $I_{D C}>300 \mu \mathrm{A}$, both $\uparrow \downarrow$ and $\downarrow \uparrow$ DWs exhibit different depinning fields with respect to difference bias currents.

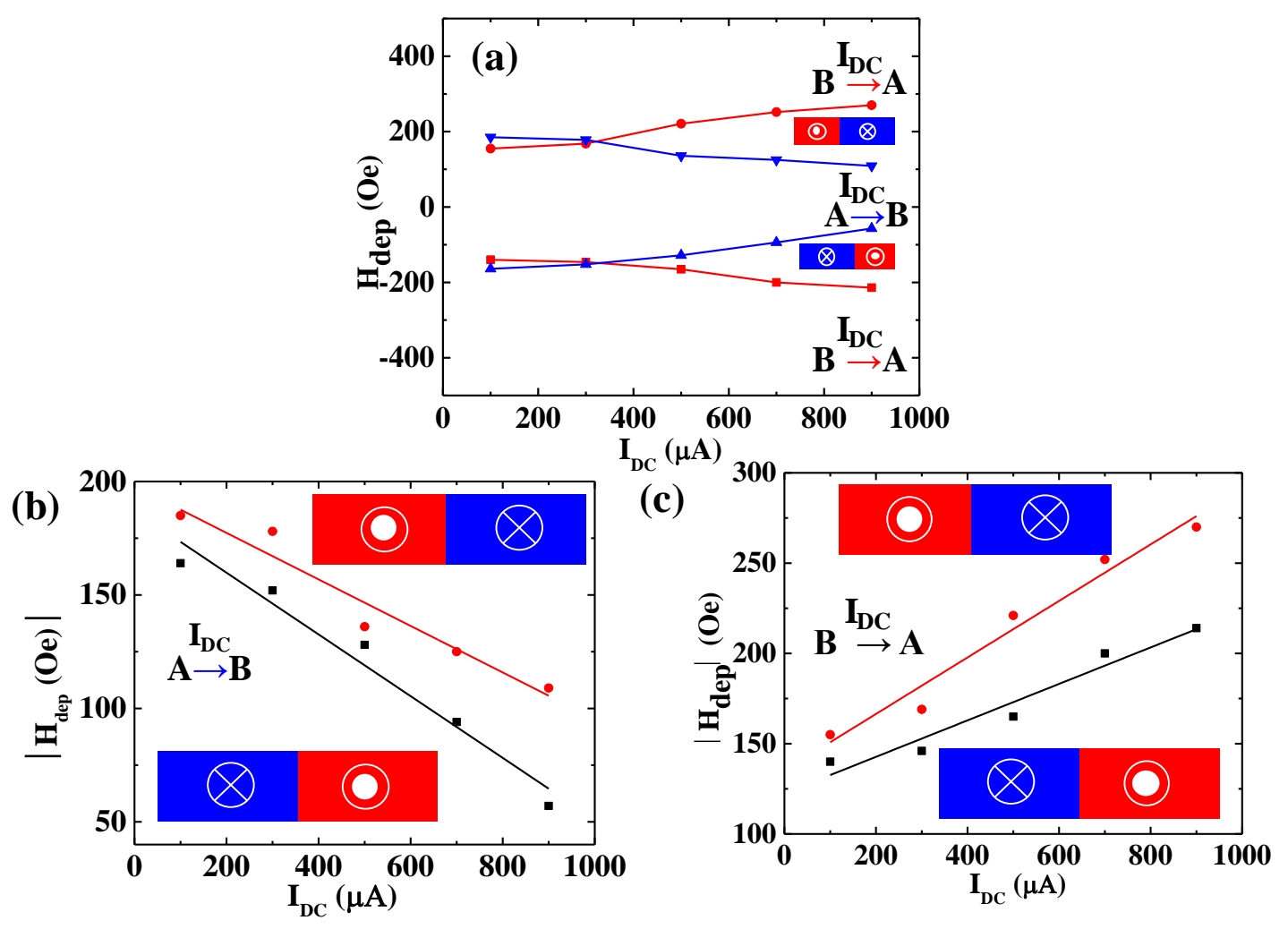

Fig. 5.11 (a) Depinning fields of $\uparrow \downarrow$ and $\downarrow \uparrow D W s$ as a function of DC bias current applied along $\mathrm{A} \rightarrow \mathrm{B}$ and $\mathrm{B} \rightarrow \mathrm{A}$. (c) Shows magnitude of the depinning field as a function of currents applied along $A \rightarrow B(b)$ and $B \rightarrow A(c)$.

Fig 5.11 (b) shows the magnitude of the depinning fields for $\uparrow \downarrow$ and $\downarrow \uparrow$ DWs as a function of the current applied from $\mathrm{A} \rightarrow \mathrm{B}$. For both $\uparrow \downarrow$ and $\downarrow \uparrow \mathrm{DWs}$, the current assist 
the DW propagation. Interestingly, distinct depinning fields are observed for $\uparrow \downarrow$ and $\downarrow \uparrow$ DWs. As the current is increased, the difference in the depinning fields also increases. Similarly, Fig. 5.11 (b) shows the magnitude of the depinning fields for $\uparrow \downarrow$ and $\downarrow \uparrow$ DWs as a function of the current applied from $\mathrm{B} \rightarrow \mathrm{A}$. The current is hindering the $\mathrm{DW}$ propagation. Like the previous case, the difference in the depinning fields for $\uparrow \downarrow$ and $\downarrow \uparrow \mathrm{DW}$ were found to increase with DC bias current. In our sample stack, the left handed Nèel DW is the stable DW configuration as demonstrated by the DMI measurement. Fig. 5.12 illustrates the directions of the current induced effective fields $\left(H_{S L}, H_{F L}\right)$ acting on the left handed Nèel DW of both $\uparrow \downarrow$ and $\downarrow \uparrow$. For a $\uparrow \downarrow$ DW with its internal magnetization stabilized along the $-x$ direction, the effective field due to the Slonczewski-like torque should be aligned along $\left(m \times\left(z \times j_{e}\right)\right)$ or $+z$ direction.

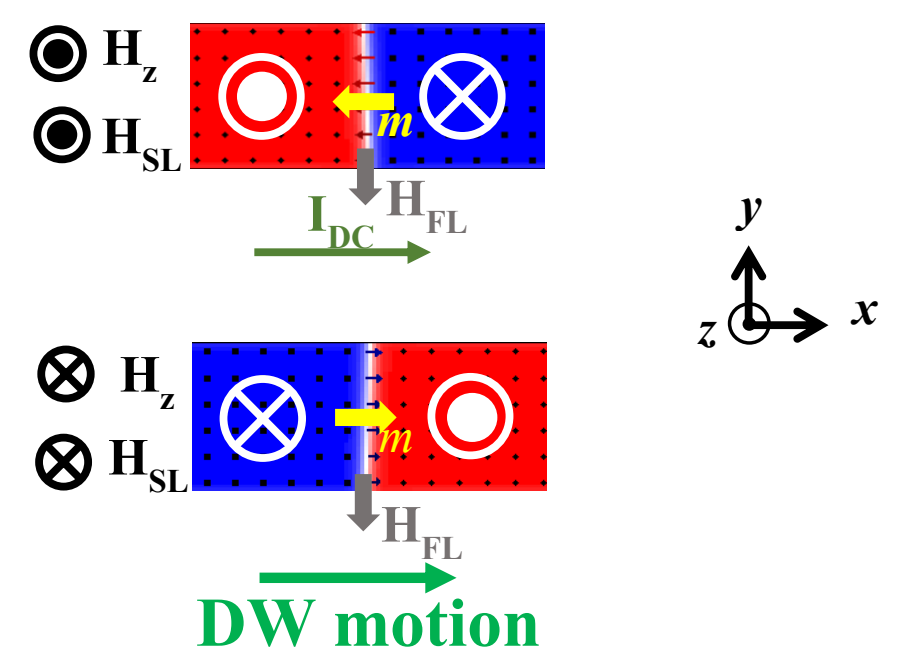

Fig. 5.12 Schematic of the directions of the current induced effective fields () acting on the left handed Nèel DWs of both $\uparrow \downarrow$ and $\downarrow \uparrow$.

Similarly, for a $\downarrow \uparrow \mathrm{DW}, \mathrm{H}_{\text {SL }}$ should be oriented along the $-z$ direction. For these two DWs, the applied out-of-plane field $\left(H_{z}\right)$ and Slonczewski-like effective field $\left(H_{S L}\right)$ act in 
concert that results in the same depinning field. The observed asymmetry in the depinning fields for $\uparrow \downarrow$ and $\downarrow \uparrow$ DWs thus cannot be ascribed to the $H_{S L}$. Different devices have been tested and confirmed that this depinning anomaly is not due to structural irregularities and edge defects. Our AC harmonic measurements reveal that the magnitude of the current induced effective field, $H_{F L}=\sim 7$ Oe per $10^{11} \mathrm{Am}^{-2}$, due to field-like torque is comparable to the $H_{S L}=\sim 14$ Oe per $10^{11} \mathrm{Am}^{-2}$. The $H_{F L}$ can be described simply by an external magnetic field that lies in-plane and transverse to the current direction. The magnitude of $H_{F L}$ scales linearly with current. Unlike $H_{S L}, H_{F L}$ does not contribute to the DW motion but it alters the DW internal magnetization. $H_{F L}$ can significantly contribute to the rotation of the DW moment. As shown in Fig. 5.12, the direction of $H_{F L}$ is fixed along $\left(z \times j_{e}\right)$ or $+y$ axis for both $\uparrow \downarrow$ and $\downarrow \uparrow$ DWs. The resultant Zeeman interaction tilts the DW moment in the same direction as the DW propagation for $\downarrow \uparrow \mathrm{DW}$, while the Zeeman interaction tilts the DW moment in the direction opposite to the current direction for $\uparrow \downarrow$ DW. The strength of $H_{F L}$ increases with higher applied current which in turn enlarge the degree of DW tilt in the respective directions for $\uparrow \downarrow$ and $\downarrow \uparrow$ DWs. Consequently, $\downarrow \uparrow$ DW have low depinning field compared to that of the $\uparrow \downarrow \mathrm{DW}$.

\subsubsection{Direct observation of DMI induced domain wall tilting}

Ex-situ polar Kerr microscopy was employed to obtain DW configuration after it depins from the $\mathrm{HC}_{\mathrm{I}}$. For this measurement, we chose a $1 \mu \mathrm{m}$ wide $\mathrm{NW}$ for ease of imaging and the Hall crosses are separated by a distance of $10 \mu \mathrm{m} . I_{D C}$ of $600 \mu \mathrm{A}$ was applied in the direction shown in the Fig. 5.13 (a). The magnetically saturated state with field applied along the $+z$ orientation is shown in Fig 5.13 (a). A DW was nucleated in the nanowire and simultaneous application of a perpendicular magnetic field sweeping along the negative 
$(-z)$ direction together with $I_{D C}$ was used to drive the DW. Fig. 5.13 (b) shows the Kerr image of the DW after it depins from the $\mathrm{HC}_{\mathrm{I}}$. The DW tilting can be clearly seen (dotted square) as it propagates along the NW under the application of perpendicular field and current. It was also observed that the branches of the Hall cross I were not completely switched. This confirms that the current dominates DW depinning process at the Hall cross.

(a)

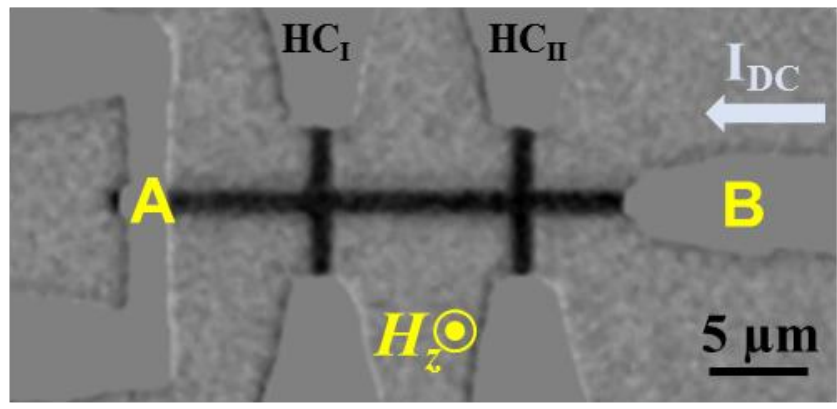

(b)

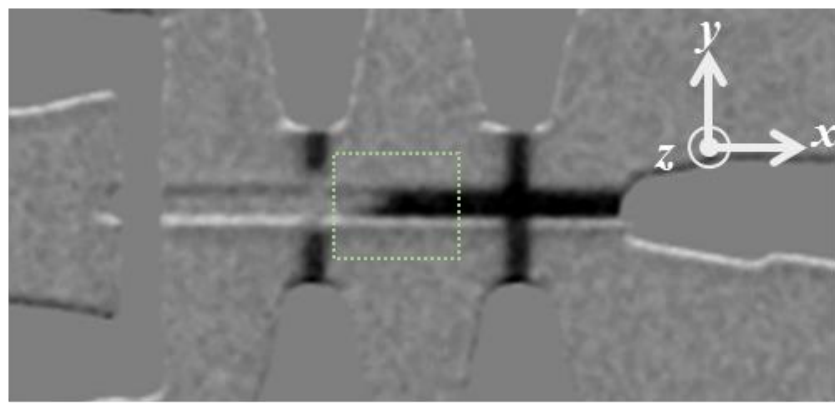

(c)

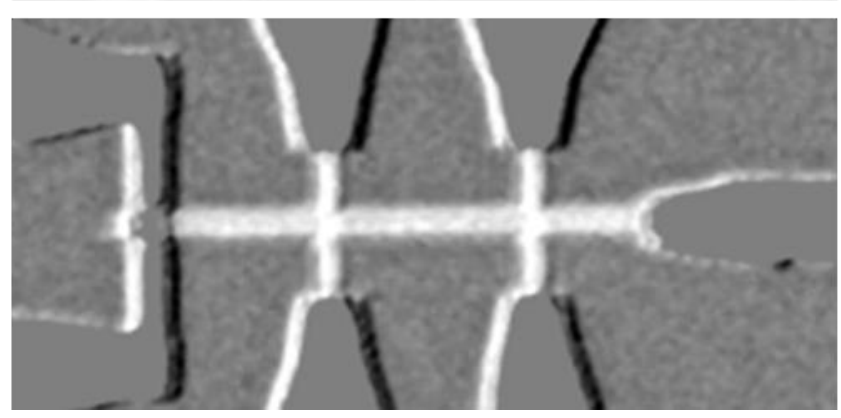

Fig. 5.13 Kerr imaging of the magnetic DW configuration for the current-assisted field driven DW motion. (a) Initial magnetization state of the NW. (b) DW tilting as it passes through the $H C_{I .}$ (c) Magnetization state of the NW after the DW propagated to the other end of the NW. 
When the perpendicular field was further increased, the DW moved to the other end of the NW and results in complete switching of the NW in the opposite direction, as shown in Fig. 5.13 (c).

\subsubsection{Effect of Hall cross spacing on DW depinning}

In order to see the effect of DW tilting on the depinning process electrically, depinning measurements in nanowires with different Hall cross spacing for a fixed nanowire width were investigated. For a Hall cross spacing of $3 \mu \mathrm{m}$, Fig. 5.14 (a, b) shows normalized $\mathrm{R}_{\mathrm{Hall}}$ measured across $\mathrm{HC}_{\mathrm{I}}$ and $\mathrm{HC}_{\mathrm{II}}$ as a function of magnetic field for various $I_{D C}$ applied from $\mathrm{B} \rightarrow \mathrm{A}$. As the magnetic field was swept in the negative $(-z)$ direction, two steps in $\mathrm{R}^{\mathrm{I}}$ Hall were observed. The first step corresponds to the DW reaching the left part of the Hall cross while the second corresponds to the DW depinning from the Hall cross I.

As the $\mathrm{I}_{\mathrm{DC}}$ was increased from $100 \mu \mathrm{A}$ to $900 \mu \mathrm{A}$, the magnitude of the depinning field increases from -170 Oe to -226 Oe. Similarly, for $\mathrm{HC}_{\mathrm{II}}$, the same switching behavior as a function of the $I_{D C}$ is observed. For $I_{D C}<700 \mu \mathrm{A}$, the DW depins at the same field for both $\mathrm{HC}_{\mathrm{I}}$ and $\mathrm{HC}_{\mathrm{II}}$. Interestingly, for $\mathrm{I}_{\mathrm{DC}} \geq 700 \mu \mathrm{A}$, the $\mathrm{DW}$ depins from $\mathrm{HC}_{\mathrm{II}}$ at a lower field of -210 Oe as compared to that of $\mathrm{HC}_{\mathrm{I}}$ at -225 Oe as can be clearly see in the inset of Fig. 5.14 (b). In contrast, for larger Hall cross spacing $(7 \mu \mathrm{m})$, the DW depins from $\mathrm{HC}_{\mathrm{I}}$ completely before it starts to depin form $\mathrm{HC}_{\mathrm{II}}$ as shown in Fig. 5.14 (c, d). The change in depinning field across the two Hall crosses may be attributed to the DMI induced DW tilting. 


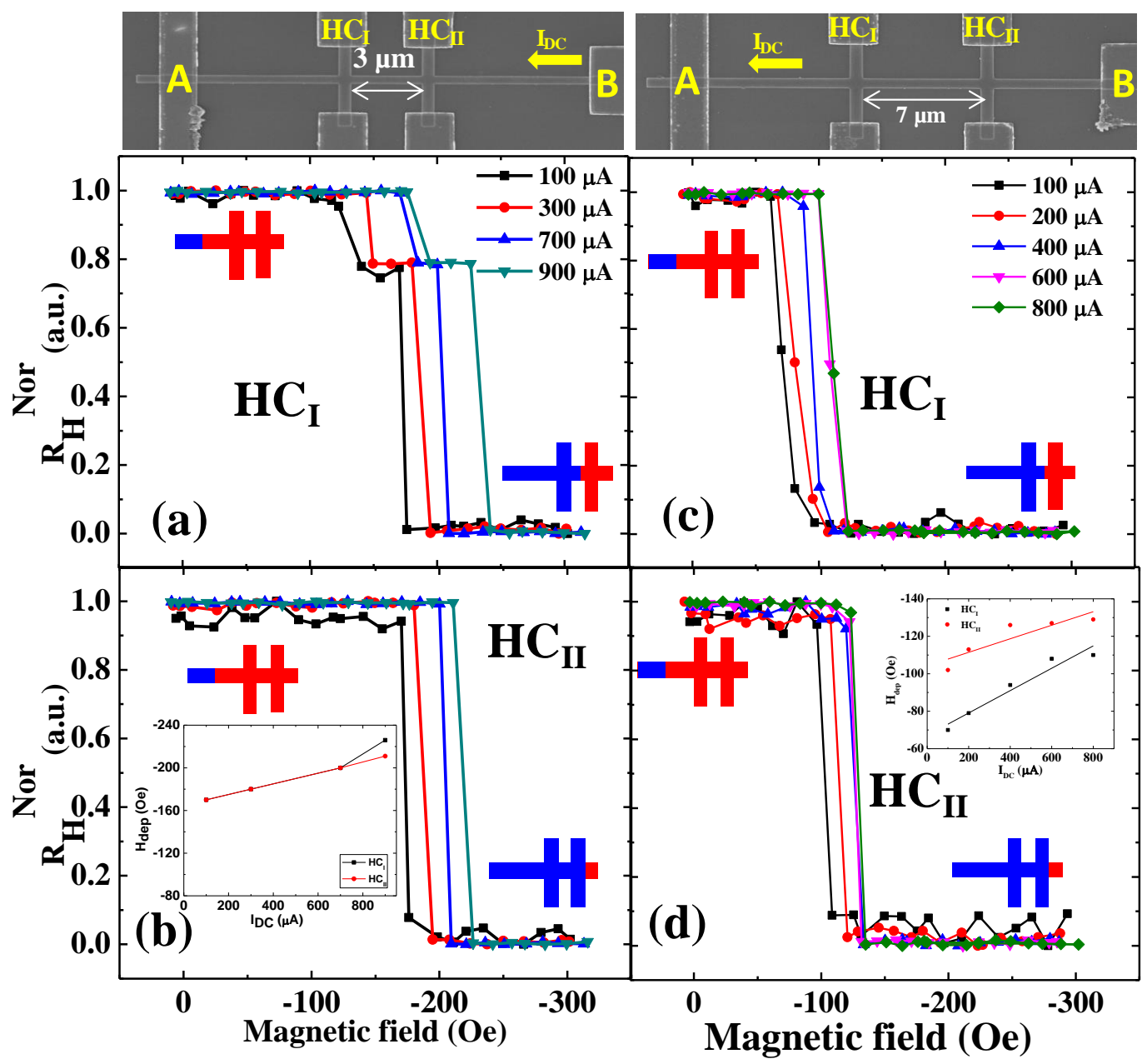

Fig. 5.14 Current assisted field driven DW depinning for $300 \mathrm{~nm}$ wide NWs with Hall crosses separated by a distance of $d=3 \mu \mathrm{m}, 7 \mu \mathrm{m}$. (a) and (b) correspond to Normalized

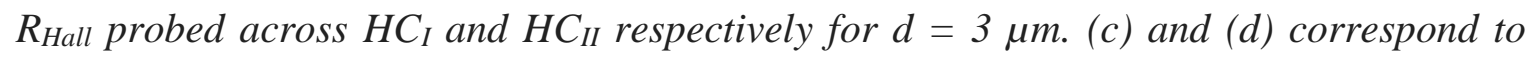
respective Normalized $R_{\text {Hall }}$ measured across $H C_{I}$ and $H C_{I I}$ for $d=7 \mu \mathrm{m}$.

\subsubsection{Dependence of the nanowire width on depinning field}

Additionally, effect of nanowire width on DW depinning field was studied. Fig. 5.15 shows the depinning field $\left(H_{d e p}\right)$ as a function of DC bias current for different wire widths $(w=300,500$ and $800 \mathrm{~nm})$. An out-of-plane field of $-H_{z}$ was swept together with a DC bias current applied in the direction opposite to the field induced DW motion. For each wire 
width, as expected, the depinning field $\left(H_{d e p}\right)$ increases linearly with $\mathrm{I}_{\mathrm{DC}}$ suggesting that the current is hindering the DW motion.

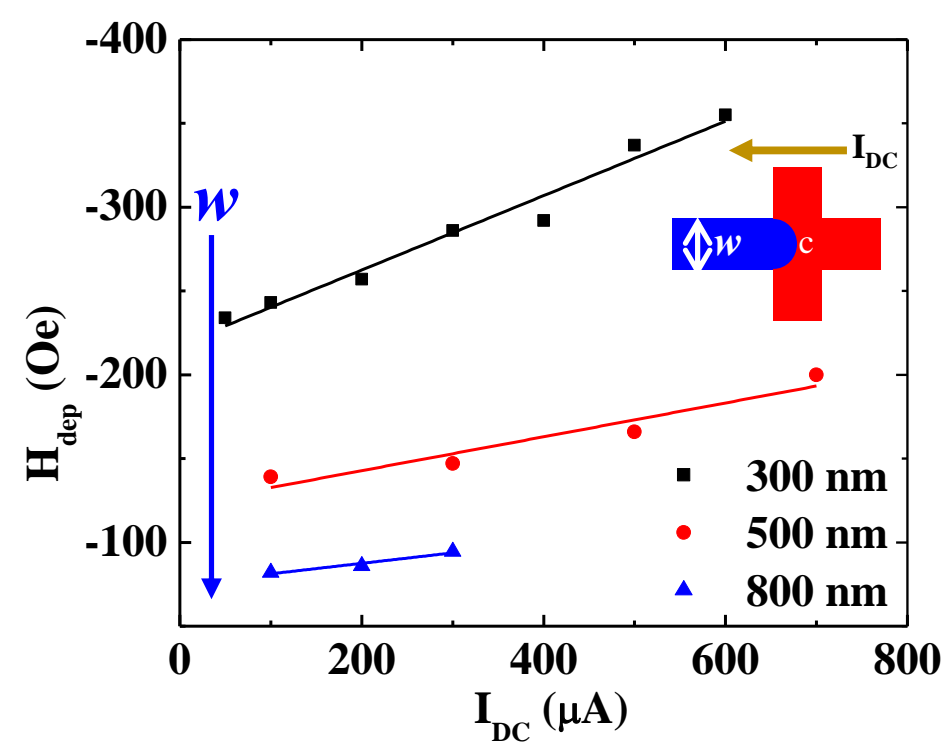

Fig. $5.15 H_{d e p}$ as a function of DC bias current, $I_{D C}$ for various $N W$ widths $(w=300,500$ and $800 \mathrm{~nm}$ ). Inset shows the schematic of the Hall cross structure where $w$ denotes the width of the NW and $C$ denotes the center of the Hall cross.

For a fixed DC bias current, the depinning field decreases with the increase of the wire width. For a DW moving through the Hall cross, the depinning field [31-32] can be expressed as

$$
H_{p}(x)=\frac{\sigma_{w}}{2 M_{s}}\left(\frac{\partial l_{w}}{\partial S}\right)
$$

where $\sigma_{w}$ is the DW energy density, $M_{s}$ is the saturation magnetization, $l_{w}$ is the DW length and $S$ is the area covered by the domain moving into the Hall cross. The maximum propagation field is given by

$$
H_{p, \max }=\frac{\sigma}{w M_{s}}
$$


and is found to be maximum at the cross center $C$, as depicted in inset of Fig. 5.15, where $w$ is the width of the NW. The shape of the DW within the Hall cross is governed by the competition between DW energy and Zeeman energy. This leads to a circular arc-shape wave front for the DW within the Hall cross as shown in the inset. As the width of the NW is reduced, from eqn. 5.8, the propagation field at the center of the Hall cross is expected to increase.

To further understand the DW depinning through Hall cross structures, micromagnetic simulations have been performed. The parameters used in the simulation are: Saturation magnetization, $M_{s}=600 \times 10^{5} \mathrm{~A} / \mathrm{m}$, exchange constant, $\left(A_{e x}\right)=15 \mathrm{pJ} / \mathrm{m}^{2}$, anisotropy constant, $k=5 \times 10^{11} \mathrm{~A} / \mathrm{m}$, DMI constant, $D=0.7 \mathrm{~mJ} / \mathrm{m}^{2}$, current density $j_{e}=5$ $\times 10^{11} \mathrm{~A} / \mathrm{m}^{2}$, spin Hall angle, $\theta_{S H E}=0.12$.

Fig. 5.16 shows spin state evolution obtained from micromagnetic simulation as the DW is driven by an out-of-plane field together with a DC bias current. The presence of DMI results in DW tilting as it propagates along the nanowire [14].

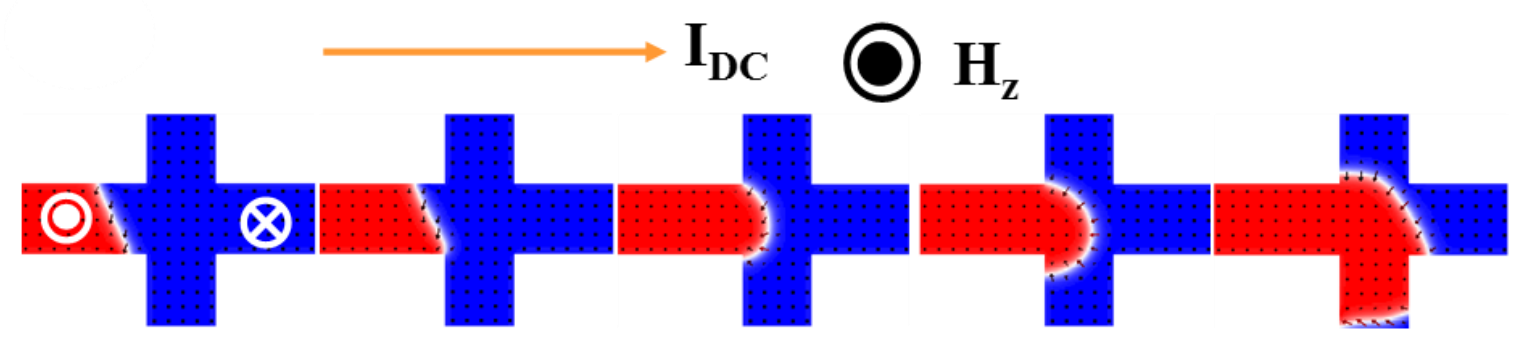

Fig. 5.16 Spin state evolution obtained from micromagnetic simulation as DW traverses through a Hall cross structure. DC bias current and out-of-plane magnetic field are applied simultaneously through the nanowire. 
The shape of the DW within the Hall cross results from the competition between the DW energy, which tends to reduce the DW length and Zeeman energy, which increases the area to be reversed [25]. For a field dominated depinning process, as the Zeeman energy increases, the DW depinning through the Hall cross is symmetrical with respect to upper (U) and lower (L) edges of the Hall cross. However, for DC current assisted DW depinning, the DW follows different phenomenon. When the tilted DW reaches the Hall cross, it is pinned at the upper edge (U) of the Hall cross as seen in Fig 5.16. As the Zeeman energy increases further, the DW depins from lower edge of the nanowire and expands within the Hall cross, while still being pinned at the upper edge of the nanowire. The lower edge of the DW propagates through the Hall cross and exits into the nanowire. As the energy of the DW is proportional to its length, the increase in DW energy allows the DW to depin from the upper edge of the Hall cross. Subsequently, the energy minimization leads to the DW regaining its original shape as it exits from the Hall cross.

\subsection{Discussion}

Spin-orbit torque driven DW motion in perpendicularly magnetized materials has attracted lot of interest not only due to the rich physics in the HM/FM heterostructures but also due to possible potential low energy applications for magnetic memory and logic devices [8-9]. Depending on the type of heavy metal in the HM/FM heterostructures, the spin-orbit torques induces DW motion either along the current flow direction or opposite to the current flow direction. It has been reported that in the absence of an in-plane field, SOT drives up-down and down-up DWs identically in the same direction. i.e. they have same depinning fields for fixed current density. Interestingly, in this thesis, for field dominated DW driving, identical depinning fields were observed while for spin-orbit torque dominated 
DW driving, distinct depinning for up-down and down-up were observed. Further, for current dominated DW depining, different depinning efficiencies for Up-Down and DownUp DWs were observed and the difference was found to increase with increasing the magnitude of the DC bias current. The interplay between the SOT and DMI is attributed to the observed experimental results.

\subsection{Summary}

To summarize, DMI induced chiral Néel DW depinning driven by SOT in perpendicularly magnetized $\mathrm{Co} / \mathrm{Ni}$ nanowires was investigated. A cross-over from field dominated DW depinning to current dominated DW depinning was observed as the magnitude of the current was increased. For field dominated DW depinning, both Up-Down and Down-Up DWs exhibit same depinning efficiencies. However, for current dominated DW depinning, current induced SOTs were estimated and found that the sample shows appreciable magnitudes for both Slonczewski-like and field-like effective fields. Further, it was shown that the presence of finite Dzyaloshinskii-Moriya interaction (DMI) stabilizes

left handed chiral Néel DW in the stack. The interplay between SOT and DMI was attributed to the observed depinning anomaly. 


\section{References}

1. S. Parkin, M. Hayashi and L. Thomas, Science320, 190 (2008).

2. D. A. Allwood, G. Xiong, C. C. Faulkner, D. Atkinson, D. Petit and R. P. Cowburn, Science 309, 209 (2008).

3. S. Goolaup, M. Ramu, C. Murapaka and W. S. Lew, Sci. Rep.5, 9603 (2015).

4. L. Berger, J. Appl. Phys.55 1954 (1984).

5. J. Slonczweski, J. Magn. Magn. Mater.159 L1 (1996).

6. L. Berger, J. Appl. Phys.71, 2721 (1992).

7. G. Tatara, H. Kohno, Phys. Rev. Lett.92, 086601 (2004).

8. T. Moriya, Phys. Rev. Lett. 4, 228 (1960).

9. M. Heide, G. Bihlmayer, S. Blügel, Phys. Rev. B 78, 140403(2008).

10. A. Thiaville, S. Rohart, E. Jue, V. Cros and A. Fert, Europhys.Lett. 100, 57002 (2012)

11. S. Emori, U. Bauer, S. M. Ahn, E. Martinez, and G. S. D. Beach, Nat. Mater.12, $611(2013)$

12. K. S. Ryu, L. Thomas, S. H. Yang, and S. S. Parkin, Nat. Nanotechnol.8, 527 (2013).

13. S. G. Je, D.H. Kim, S. C. Yoo, B. C. Min, K. J. Lee, and S.B. Choe, Phys. Rev. B 88, 214401 (2013).

14. O. Boulle, S. Rohart,L. D. B. Prejbeanu, E. Jue, I. M. Miron, S. Pizzini, J. Vogel, G. Gaudin and A. Thiaville, Phys. Rev. Lett.111 2172013 (2013).

15. S. Emori et al., Phys. Rev. B90 184427 (2014).

16. L. Liu, C.-F. Pai, Y. Li, H. W. Tseng, D. C. Ralph, and R. A. Buhrman, Science 336, 555 (2012). 
17. P. P. J. Haazen, E. Mure, J. H. Franken, R. Lavrijsen, H. J. M. Swagten and B. Koopmans, Nature Mater.12, 299 (2013).

18. K. W. Kim, S. M. Seo, J. Ryu, K. J. Lee, and H. W. Lee, Phys. Rev. B85, 180404 (2012).

19. T. A. Moore, I. M. Miron, G. Gaudin, G. Serret, S. Auffret, B. Rodmacq, A. Schuhl, S. Pizzini, J. Vogel, and M. Bonfim, Appl. Phys. Lett. 93, 262504 (2008).

20. I. M. Miron, P.-J. Zermatten, G. Gaudin, S. Auffret, B. Rodmacq, and A. Schuhl, Phys. Rev. Lett. 102, 137202 (2009).

21. I. M. Miron, G. Gaudin, S. Auffret, B. Rodmacq, A. Schuhl, S. Pizzini, J. Vogel, and P. Gambardella, Nat. Mater. 9, 230 (2010).

22. U. H. Pi, K. W. Kim, J. Y. Bae, S. C. Lee, Y. J. Cho, K. S. Kim, and S. Seo, Appl. Phys. Lett. 97, 162507 (2010).

23. I. M. Miron, T. A. Moore, H. Szambolics, L. D. Buda-Prejbeanu, S. Auffret, B. Rodmacq, S. Pizzini, J. Vogel, M. Bonfim, A. Schuhl, and G. Gaudin, Nat. Mater. 10, 419 (2011).

24. I. M. Miron, K. Garello, G. Gaudin, P.-J. Zermatten, M. V. Costache, S. Auffret, S. Bandiera, B. Rodmacq, A. Schuhl, and P. Gambardella, Nature (London) 476, 189 (2011).

25. A. V. Khvalkovskiy, V. Cros, D. Apalkov, V. Nikitin, M. Krounbi, K. A. Zvezdin, A. Anane, J. Grollier and A. Fert, Phys. Rev. B 87, 020402 (2013).

26. T. Koyama, D. Chiba, K. Ueda, H. Tanigawa, S. Fukami, T. Suzuki, N. Ohshima, N. Ishiwata, N. Nakatani, and T.One, Appl. Phys. Lett. 98192509 (2011). 
27. K. Y. Wang, A. C. Irvine, J. Wunderlich, K. W. Edmonds, A. W. Rushforth, R. P. Campion, C. T. Foxon, D. A. Williams and B. L. Gallagher, New J. Phys.10 085007 (2008)

28. S. V. Tarasenko, A. Stankiewicz, V. V. Tarasenko, and J. Ferre, J. Magn. Magn. Mater.189, 19 (1998).

29. X. Qiu, W. Legrand, P. He, Y. Wu, J. Yu, R. Ramaswamy, A. Manchon and H. Yang, Phys. Rev. Lett. 117, 217206 (2016).

30. J. Kim, J. Sinha, M. Hayashi, M. Yamanouchi, S. Fukami, T. Suzuki, S. Mitani, and H. Ohno, Nature Mater. 12, 240 (2013).

31. J. Wunderlich, D. Ravelosona, C. Chappert, F. Cayssol, V. Mathet, J. Ferre, J. P, Jamet, and A. Thiaville, IEEE Trans. Magn.37 4 (2001). 


\section{Chapter 6}

\section{Conclusions and Outlook}

In the course of this study, controlled Néel DW manipulation and dynamics in both in-plane and out-of-plane magnetic anisotropy materials has been carried out. The DW dynamics are studied under the influence of both magnetic field and spin polarized current. Direct observation of the magnetization configurations was obtained by using MFM. To substantiate the experimental results, micromagnetic simulations have been performed.

\subsection{Conclusion}

Firstly, a novel scheme that controls the internal profile of a transverse DW was proposed and verified using MFM measurement. The processing of information can be encoded in the internal profile of DWs, paving way for future non-volatile logic devices. This is achieved by engineering the nanostructures that provides deterministic logic operations on the chirality of the transverse DW. The device is clocked via linear magnetic field. A rectifier, regardless of the input transverse DW, provides a predetermined output. While the inverter provides an output which is complimentary to the input DW. The slight variations in the fabricated structures does not affect the rectification/inversion operation. It implies that the device is robust and can be applied for logical applications. The experimental results were further substantiated with the micromagnetic simulations. For the devices considered in this thesis, the length of the nanowire between a nucleation pad and the detector is kept at $200 \mathrm{~nm}$, which is well below the reported fidelity length. It ensures that the integrity of input and output DWs are not compromised.

Secondly, a method to generate single DW with $100 \%$ probability on a nanosecond time scale was presented. This method exploits the stray field magnetostatic interaction 
between DWs and edge of the nanowire. DWs interaction in in-plane magnetized nanowires were investigated for controlled DW generation. The existing methods requires either long current pulses that stabilizes the transverse DW or global magnetic fields that separates nucleated DWs farther apart by eliminating the DW interaction. Global magnetic field has scalability issues while large amplitude currents cause Joule heating effect. By contrast, the new scheme requires single pulse for the deterministic generation of single DW. The DW interaction has further investigated from the stand point of device application. When two DWs of opposite edge defects interact each other, spin waves are emitted. The spin wave characteristic properties such as frequency and amplitude as a function of external magnetic field and geometrical dimensions of the nanowire were studied. The amplitude of the spin wave mode is found to increase with increasing field and the spin wave power has the maximum amplitude along the middle of the nanowire width. The decay coefficient of the spin wave power suggests that the power drops to $10 \%$ at a distance of $350 \mathrm{~nm}$ away from the annihilation point, which limits the spacing between two adjacent DWs.

Lastly, Spin-orbit torque driven DW motion in perpendicularly magnetized materials has attracted lot of interest due to possible potential low energy applications for magnetic memory and logic devices. Depending on the type of heavy metal in the HM/FM heterostructures, the spin-orbit torques induces DW motion either along the current flow direction or opposite to the current flow direction. It has been reported that in the absence of an in-plane field, SOT drives up-down and down-up DWs identically in the same direction. i.e. they have same depinning fields for fixed current density. Interestingly, in this thesis, for field dominated DW driving, identical depinning fields were observed while for spin-orbit torque dominated DW driving, distinct depinning for up-down and down-up 
were observed. Further, for current dominated DW depining, different depinning efficiencies for Up-Down and Down-Up DWs were observed and the difference was found to increase with increasing the magnitude of the DC bias current. The interplay between the SOT and DMI is attributed to the observed experimental results.

\subsection{Future Outlook}

\subsubsection{Chiral magnetic textures}

In some thin films with broken inversion symmetry, an additional interaction known as the Dzyaloshinskii-Moriya interaction (DMI) can be dominant and it introduces chiral magnetic textures [1]. The chiral magnetic textures include both DWs and Skyrmions have potentially useful for low-power device operations. Skyrmions are vortex like swirls of magnetization as show in Fig. 6.1. Skyrmion based devices have the potential for ultrahigh package density, high speed, non-volatality, high speed [2-4]. The first feature is due to the small sizes of the Skyrmions.

DMI is known to originate from the strong spin-orbit coupling. In chapter 5, DMI has been quantified using asymmetric domain expansion under the influence of both inplane and out-of-plane magnetic fields. For the samples considered, the Néel DWs are stabilized under zero applied field. However, the observations of these magnetic textures are limited to epitaxial grown ultrathin layers of ferromagnetic materials on heavy metals and requires advanced characterization tools, spin-polarized scanning tunneling microscopy, spin polarized low energy electron microscopy (SPEEM), at high external magnetic fields and low temperatures [8-10]. 

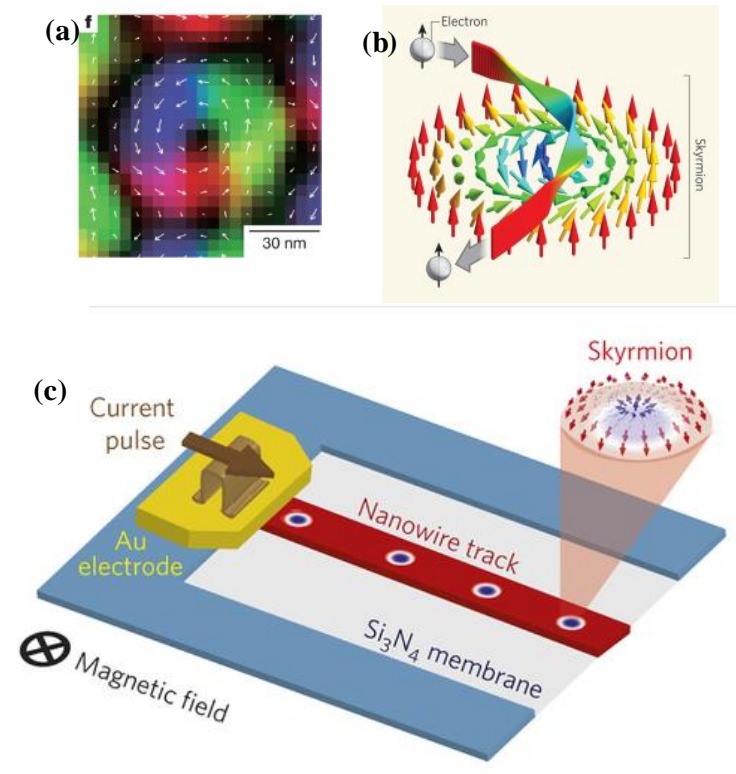

Fig. 6.1 (a) Skyrmion stabilized in a helical-magnetic crystal Fe 0.5 Co0.5Si probed with Lorentz transmission electron microscopy [5]. (b) Schematic of spin torque exerted on a Skyrmion [6]. (c) Schematic of a magnetic track with measurement setup and Skyrmions are stabilized by an out-of-plane magnetic field [7].

The presence of strong DMI in materials, which are readily fabricated and probed at room temperature have vast applications, beyond the development of DW based memory and logic devices. In this thesis, the presence of DMI in sputter deposited Co/Ni multilayers has been quantified using wide-field Kerr microscopy. It will be extremely useful to find the right combination of ferromagnetic elements and heavy metals, which gives strong DMI. Additionally, the growth conditions such as composition and thickness of the layers can be tuned in such a way that it gives strong DMI.

After stabilizing the Skyrmions by tuning the growth conditions, the Skyrmions can be driven by spin current. Recently, it has been proposed that the spin current from spin Hall effect can drive DWs much faster as compared to conventional spin transfer torque. The efficiency of the generation of spin current is governed by the spin Hall angle. The spin 
Hall angle can be defined as a ratio between the spin current to the charge current injected into the material [11]. By proper selection of materials, the spin Hall angle can be tuned and it results in efficient spin torque.

Fig. 6.2 shows a schematic of a Skyrmion based racetrack with measurement set up. Skyrmions can be nucleated via local heating, magnetic fields and electrical current [1217]. Fundamentally, a Skyrmion can be created by overcoming the potential barrier that promotes a topological transition of the magnetic textures. A simple method to create a Skyrmion is change to change the strength of the magnetic field of the material.

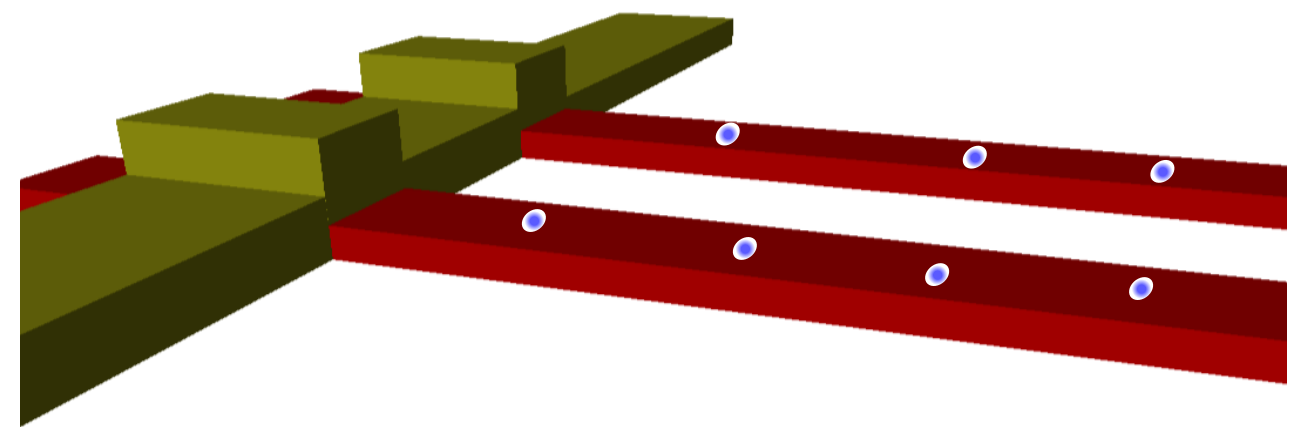

Fig. 6.2 Schematic of Skyrmion based nanotrack memory.

After Skyrmions are injected in the racetrack, they can be driven by spin transfer torque (STT) or spin Hall effect (SHE) [18-21]. For STT induced Skyrmion motion, spin polarized current is injected in the plane of the sample while for SHE induced Skyrmion motion, the spin polarized current is injected in the vertical direction from heavy metal, where the spin current is generated by passing charge current through it. The motion can be controlled by varying the current density and pulse duration. 
As part of Skyrmion detection, the small size of the Skyrmions make it difficult to detect reliably with a high signal-to-noise ratio. The solution lies in tuning the material properties such that high TMR ratio can be attained or developing novel detection methods.

\subsubsection{Spin wave based devices}

From the technological perspective, spin waves can be used in high speed signal transmission and spin based logic devices [22-24]. Recently, it was shown that the spin current from spin waves can be used to induce domain wall motion [25-26]. Spin waves have very high frequencies in the range of few tens of GHZ. Fundamentally spin waves are produced as a result of exchange interaction of spins in ferromagnets.

In this thesis, spin wave emission due to domain wall collision was studied. It was found that the spin wave characteristics such as frequency and amplitude are strongly influenced by the geometrical parameters of the nanostructures and external field strength [27]. The decay coefficient of the spin wave power suggests that the power drops to $10 \%$ at a distance of $350 \mathrm{~nm}$ away from the collision point, implying the attenuating nature of spin waves.

The key challenging for the spin waves to be employed in future spin wave based devices is the attenuation of the spin waves. It implies that the spin wave amplitude drops exponentially, which makes unreliable for the spin waves to be used in the devices. This is due to the intrinsic damping of the ferromagnetic materials. The intensity of the damping can be measured form the damping constant, $\alpha$. The simple ferromagnetic material, permalloy, has a damping constant of $\sim 0.01$, which is considered to be high [28-29]. 
Materials with low damping constants can overcome this issue. Materials with low damping constant have been investigated. To date, Yttrium iron garnet ( $\mathrm{YIG}$ : $\mathrm{Y}_{3} \mathrm{Fe}_{5} \mathrm{O}_{12}$ ) is the lowest damping material [30-34]. The future studies would focus on replacing the conventional ferromagnetic materials with low damping materials and create spin wave based logic devices.

Additionally, spin wave generation in perpendicularly magnetized materials would lead to new pathways. Perpendicularly magnetic anisotropy materials are known to have large anisotropy energy, small domain wall width and high thermal stability. Further, recent observations of spin-orbit torques and peculiar magnetic textures in perpendicularly magnetized materials have provided greater possibility for future spintronic devices. As such, it would be extremely useful for the spin waves to be harvesting in perpendicularly magnetized materials. 


\section{References}

1. A. Tretiakov, A. Abanov, Phys. Rev. Lett. 105, 157201 (2010)

2. M. Heide, G. Bihlmayer, and S. Blügel, Phys. Rev. B 78, 140403 (2008).

3. E. Martinez, S. Emori, and G. S. D. Beach, Appl. Phys. Lett. 103, 072406 (2013).

4. A. Thiaville, S. Rohart, E. Jue, V. Cros, A. Fert, Euro Phys. Lett. 100, 57002 (2012).

5. X. Z. Yu, Y. Onose, N. Kanazawa, J. H. Park, J. H. Han, Y. Matsui, N. Nagaosa and Y. Tokura, Nature 465, 901 (2010).

6. C. Pflediderer, A. Rosch, Nature 465, 880 (2010)

7. S. Woo, K. Litzius, B Kruger, M. Y. Im, L. Caretta, K. Richter, M. Mann, A. Krone, R. M. Reeve, M. Weigand, P. Agarwal, I. Lemesh, M. A. Mawass, P. Fischer, M. Klaui and G. S. D. Beach, Nature Mater. 15, 501 (2016)

8. S. Heinze, Nature Phys. 7, 713 (2011).

9. M. Bode, M. Heide, K. V. Bergmann, P. Ferriani, S. Heinze, G. Bihlmayer, A. Kubetzka, O. Pietzsch, S. Blugel and R. Wiesendanger, Nature 447, 190 (2007).

10. G. Chen, J. Zhu, A. Quesada, J. Li, A. T. N’Diaye, Y. Huo, T. P. Ma, Y. Chen, H. Y. Kwon, C. Won, Z. Q. Qiu, A. K. Schmid and Y. Z. Wu, Phys. Rev. Lett. 110, $177204(2013)$

11. S. Woo, M. Mann, A. J. Tan, L. Caretta and G. S. D. Beach, Appl. Phys. Lett. 105, $212404(2014)$

12. N. Romming, C. Hanneken, M. Menzel, J. E. Bickel, B. Wolter, K. V. Bergmann, A. Kubetzka, R. Wiesendanger Science, 341, 636 (2013)

13. A. H. Bobeck, P. I. Bonyhard, and J. E. Geusic, Proc. IEEE, 63, 1176 (1975).

14. R. Wiesendanger, Nat. Rev. Mater., 1, 16044 (2016). 
15. S. Z. Lin, reprint arXiv:1510.07353, (2015).

16. Y. Tchoe and J. H. Han, Phys. Rev. B, 85, 1 (2012).

17. H. Y. Yuan and X. R. Wang, Sci. Rep., 6, 22638 (2016)

18. J. Sampaio, V. Cros, S. Rohart, A. Thiaville, and A. Fert, Nat. Nanotechnol., 8, 839 (2013).

19. R. Tomasello, E. Martinez, R. Zivieri, L. Torres, M. Carpentieri and G. Finocchio, Sci. Rep., 4, 6784 (2014).

20. J. Iwasaki, M. Mochizuki, and N. Nagaosa, Nat. Nanotechnol. 8, 742 (2013).

21. M. Stone, Phys. Rev. B 53, 16573 (1996).

22. Y. Kajiwara, K. Harii, S. Takahashi, J. Ohe, K. Uchida, M. Mizuguchi, H. Umezawa, H. Kawai, K. Ando, K. Takanashi, S. Maekawa and E. Saitoh Nature 464, 262-266 (2010).

23. S. K. Kim, K. S. Lee, D. S. Han, Appl. Phys. Lett. 95, 082507 (2009).

24. Kostylev, M. P., Serga, A. A., Schneider, T., Leven, B. \& Hillebrands, B. Spinwave logical gates. Appl. Phys. Lett. 87, 153501 (2005).

25. X. S. Wang, P. Yan, Y. H. Shen, G. E. W. Bauer, and X. R. Wang, Phys. Rev. Lett. 109, 167209 (2012).

26. M. Jamali, H. Yang, and K. J. Lee, Appl. Phys. Lett. 96, 242501 (2010).

27. M. Ramu, I. Purnama, S. Goolaup, M. Chandra Sekhar and W. S. Lew, J. Appl. Phys. 115243908 (2014)

28. J. C. Lloyd and R. S. J. Smith, Appl. Phys. 30, S274 (1959).

29. K. Yamanoi, S. Yakata, T. Kimura, T. Manago, Jpn. J. Appl. Phys. 52, 083001 (2013). 
30. A. A. Serga, A. V. Chumak, and B. Hillebrands, J. Phys. Appl. Phys. 43, 264002 (2010).

31. A, V, Chumak, P. Pirro, A. A. Serga, M. P. Kostylev, R. L. Stamps, H. Schultheiss, K. Vogt, S. J. Hermsdoerfer, B. Laegel, P. A. Beck and B. Hillebrands, Appl. Phys. Lett. 95, 262508 (2009).

32. J. Sinova, D. Culcer, Q. Niu, N. A. Sinitsyn, T. Jungwirth and A. H. MacDonald, Phys. Rev. Lett. 92, 126603 (2004).

33. E. Saitoh, M. Ueda, H. Miyajima, G. Tatara, Appl. Phys. Lett. 88, 182509 (2006).

34. T. Jungwirth, J. Wunderlich, K. Olejnik, Nat. Mater. 11, 382-390 (2012). 